DAC05, page 1

Differentiate and Deliver: Leveraging Your Partners (CEO Panel)

Chair: Jay Vleeschhouwer - Merrill Lynch

Speakers: $\quad$ Warren East - ARM Holdings, plc, Cambridge, UK

Michael J. Fister - Cadence Design Systems, Inc., San Jose, CA

Aart De Geus - Synopsys, Inc., Mountain View, CA

Walden C. Rhines - Mentor Graphics Corp., Wilsonville, OR

Jackson Hu - UMC Corp., Hsinchu, Taiwan

Rick Cassidy - TSMC, San Jose, CA

\begin{abstract}
For the past 25 years, the EDA industry has played a major role in the growth of the semiconductor industry, providing tools and services that have helped companies develop electronics products that permeate and improve every aspect of our daily lives.

As the semiconductor industry moves into the nanometer era, they face many key questions when envisioning a new product. When do they want the product to reach the market? How will that product be differentiated? Where do they develop and manufacture that product?

Less than a decade ago, these questions would have been answered completely independent of whatever EDA vendor a semiconductor company selected. However, in the nanometer era, the answers to these questions can be significantly influenced not only by EDA companies but also by the IP and pure-play foundries that make up the infrastructure of the semiconductor industry. In order to compete in a global marketplace, these companies must align their individual core competencies with those of the semiconductor industry to help IC companies create products with the optimal combination of performance, price, and time-to-market.

In this panel, the CEOs of the three major EDA vendors, along with peers from the IP and manufacturing areas discuss these fundamental changes to the semiconductor industry, and the challenges of working together to help customers successfully bring new products to market.
\end{abstract}

Jay Vleeschhouwer, a senior analyst for Merrill Lynch, will moderate a series of questions for the panelists from the customer's point of view that address how EDA, IP and pureplay foundries can impact the competitiveness of semiconductor companies and the products they develop.

Keywords: Processors, EDS, Intellectual Property, Semiconductor Fabrication, SupplierCustomer Relationships 


\title{
Logic Soft Errors in Sub-65nm Technologies Design and CAD Challenges
}

\author{
Subhasish Mitra, Tanay Karnik, Norbert Seifert, Ming Zhang \\ Intel Corporation
}

\begin{abstract}
Logic soft errors are radiation induced transient errors in sequential elements (flip-flops and latches) and combinational logic. Robust enterprise platforms in sub-65nm technologies require designs with built-in logic soft error protection. Effective logic soft error protection requires solutions to the following three problems: (1) Accurate soft error rate estimation for combinational logic networks; (2) Automated estimation of system effects of logic soft errors, and identification of regions in a design that must be protected; and, (3) New cost-effective techniques for logic soft error protection, because classical fault-tolerance techniques are very expensive.

Keywords: Architectural Vulnerability Factor, Built-In Soft Error Resilience, derating, error blocking, error detection, recovery, soft error
\end{abstract}

\section{REFERENCES}

[1] H.T. Nguyen and Y. Yagil, "A Systematic Approach to SER Estimation and Solutions", Proc. Intl. Reliability Physics Symp., pp. 60 - 70, 2003.

[2] N.J. Wang, et al., "Characterizing the Effects of Transient Faults on a High-Performance Processor Pipeline," Intl. Conf. Dependable Systems and Networks, pp. 61-70, 2004.

[3] S. Mitra, N. Seifert, M. Zhang, Q. Shi and K.S. Kim, "Robust System Design with Built-In Soft Error Resilience," IEEE Computer, Vol. 38, No. 2, pp. 43-52, Feb. 2005.

[4] K.K. Goswami, R. Iyer and L.Y. Young, "DEPEND: A Simulation-Based Environment for System Level Dependability Analysis," IEEE Trans. Computers, Jan. 1997.

[5] S.S. Mukherjee, et al., "A Systematic Methodology to Compute the Architectural Vulnerability Factors for a High-Performance Microprocessor," MICRO, 2003.

[6] P. Hazucha, et al., "Measurements and Analysis of SERTolerant Latch in a 90nm Dual Vt CMOS Process," IEEE Journal Solid State Circuits, pp. 1536-1543, Sept. 2004.

[7] T. Karnik, et al., "Impact of body bias on alpha- and neutroninduced soft error rates of flip-flops," VLSI Circuits Symp., pp. 324-325, 2004

[8] T. Calin, M. Nicolaidis, and R. Velaco, "Upset Hardened Memory Design for Submicron CMOS Technology," IEEE Trans. Nucl. Sci., Vol. 43, pp. 2874-2878, Dec. 1996.

[9] T. Karnik, et al., "Selective Node Engineering for Chip-level Soft Error Rate Improvement," VLSI Circuits Symp., pp. 204-205, 2002

[10] L. Spainhower and T. A. Gregg, "S/390 Parallel Enterprise Server G5 Fault Tolerance," IBM Journal Research \& Development., pp. 863-873, Sept./Nov. 1999.

[11] S.S. Mukherjee, M. Kontz and S. Reinhardt, "Detailed Design and Evaluation of Redundant Multithreading Alternatives," Intl. Symp. Computer Architecture, 2002.

[12] N. Oh, P.P. Shirvani and E.J. McCluskey, "Error Detection by Duplicated Instructions in Super-Scalar Processors," IEEE Trans. Reliability, pp. 63-75, March 2002.

[13] N.R. Saxena, et al., "Dependable Computing and On-line Testing in Adaptive and Reconfigurable Systems," IEEE Design and Test of Computers, pp. 29-41, Jan-Mar 2000.

[14] T. Karnik, P. Hazucha, and J. Patel, "Characterization of soft errors caused by single event upsets in CMOS processes," IEEE Trans. Dependable and Secure Computing, Vol. 1, Issue 2, pp. 128-143, April-June 2004.

[15] S. Narendra, et al., "1.1V $1 \mathrm{GHz}$ communications router with on-chip body bias in 150nm CMOS," Proc. IEEE Solid-State Circuits Conference, Volume 2, pp. 218-482, Feb 2002.

[16] N. Seifert and N. Tam, "Timing Vulnerability Factors of Sequentials", IEEE Trans. Device and Materials Reliability, Vol. 4, No. 3, p. 516-522, September 2004.

[17] N. Seifert, et al., "Radiation-Induced Clock Jitter and Race", Proc. Intl. Reliability Physics Symp., 2005.

[18] R. Baumann, "The Impact of Technology Scaling on Soft Error Rate Performance and Limits to the Efficacy of Error Correction," Proc. Intl. Electron Devices Meeting, pp. 329 - 332, 2002.

[19] M. Zhang and N.R. Shanbhag, "A Soft Error Rate Analysis Methodology," Proc. ICCAD, pp. 111 - 118, 2004. 


\title{
SEU Tolerant Device, Circuit and Processor Design
}

\author{
William Heidergott \\ General Dynamics C4 Systems, Scottsdale, Arizona, USA
}

\begin{abstract}
Development of highly reliable and available systems requires consideration of the occurrence of single event upsets, the effects they have on system performance, and strategies for their prevention and mitigation. Methods of systems engineering process and the application and validation of techniques for fault tolerance are discussed as elements in the elimination and mitigation of single event upsets.

Keywords: Radiation effects, single event upset, soft error rate, fault tolerant systems, error detection and correction coding, fault avoidance, fault masking, modular redundancy, temporal redundancy
\end{abstract}

\section{REFERENCES}

[1] Bossen, D., CMOS Soft Errors and Server Design, Radiation Induced Soft Errors in Silicon Components and Computer Systems Tutorial, IRPS 2002.

[2] Johnston, A., Mitigation Methods for Soft Errors and Related Radiation Effects in Spacecraft, Radiation Induced Soft Errors in Silicon Components and Computer Systems Tutorial, IRPS 2002.

[3] Dodd, P. and Sexton, F., Mitigation of Single- Event Effects in Mission-Critical Systems, Radiation Induced Soft Errors in Silicon Components and Computer Systems Tutorial, IRPS 2002.

[4] Normand, E., Single-Event Effects in Avionics, IEEE Transactions on Nuclear Science, Vol. 43, No. 2, April 1996, Page 461-474

[5] National Geophysical Data Center (NGDC), National Oceanic and Atmospheric Administration (NOAA), http://www.ngds.noaa.gov/stp/SOLAR/COSMIC_RAYS/cosmic.html

[6] LaBel, K., Michele Gates, M., Barth, J., Stassinopoulos, E.G., Johnston, A. and Marshall, P., Single Event Criticality Analysis (SEECA), http://flick.gsfc.nasa.gov/radhome/papers

[7] Somani, A.K. and Viadya, N.H., Understanding Fault Tolerance and Reliability, Computer, pp 45-50, (Apr 1997).

[8] Avizienis, A., Toward Systematic Design of Fault-Tolerant Systems, Computer, pp 51-58, (April 1997)

[9] Nelson, V., Fault-Tolerant Computing: Fundamental Concepts, Computer, pp 19-25, (July 1990)

[10] Mitsubishi Electric Develops High-Frequency Synchronous SRAM with Dramatically Reduced Soft Error Rate, International Solid-State Circuits Conference, Feb 1999, http://www.mitsubishichips.com/press/releases/ fsram_99.htm

[11] Vinson, J, Circuit Reliability of Memory Cells with SEU Protection, IEEE Transactions on Nuclear Science, Vol. 39, No. 6, pp 1671-1678, (December 1992)

[12] Rockett, L., An SEU Hardened CMOS Data Latch Design, IEEE Transactions on Nuclear Science, Vol. 35, No. 6, pp 1682-1687, (December 1992)

[13] Calin, T., Nicolaidis, M., Velazco, R., Upset Hardened Memory Design for Submicron CMOS Technology, IEEE Transactions on Nuclear Science, Vol. 43, No. 6, pp 2874-2878, (December 1996)

[14] Norely, M., Liu, and Whitaker, S., Low Power SEU Immune CMOS Memory Circuits, IEEE Transactions on Nuclear Science, Vol. 39, No. 6, pp 1679-1684, (December 1992)

[15] Lin, S. and Costello, D., Error Control Coding - Fundamentals and Applications, Prentice-Hall, (1983)

[16] Fujiwara, E. and Pradhan, D., Error-Control Coding in Computers, Computer, (July 1990)

[17] Chen, I. and Yen, I., Analysis of Probablistic Error Checking Procedures on Storage Systems, The Computer Journal, Vol. 38, No. 5, (1995)

[18] Peercy, M. and Banerjee, P., Fault Tolerant VSLI Systems, Proceedings of the IEEE, pp 745-758, (1993)

[19] Vaidya, N.H., Comparison of Duplex and Triplex Memory Reliability, IEEE Transactions on Computers, Vol. 45, No. 4, pp 503-507, (1996)

[20] Mavis, D. and Eaton, P., Temporally Redundant Latch for Preventing Single Event Disruptions in Sequential Integrated Circuits, Mission Research Corporation Technical Report P8111.29, October 1998

[21] Sosnowski, J., Transient Fault Tolerance in Digital Systems, IEEE Micro, (1994)

[22] McMillin, B., Fault Tolerance for Multicomputers: The Application Oriented Paradigm, Department of Computer Science, University of Missouri-Rolla, (1997) 
[23] Banatre, M. and Lee, P., Hardware and Software Architectures for Fault Tolerance, Experiences and Perspectives

[24] Kim, K.H., and Welch, H.O., Distributed Execution of Recovery Blocks: An Approach for Uniform Treatment of Hardware and Software Faults in Real-Time Applications, Fault-Tolerance Systems: Techniques and Applications, edited by Hoang Phan, pp 95-105, (1992)

[25] Blaquiere, Y., Gagne, G., Savaria, Y., and Evequoz, C., A New Efficient Algorithm-Based SEU Tolerant System Architecture, IEEE Transactions on Nuclear Science, Vol. 42, No. 6, (Dec 1995) 
DAC05, pages 11-16

\title{
Variability and Energy Awareness: A Microarchitecture-Level Perspective
}

\section{Diana Marculescu, Emil Talpes}

Dept. of Electrical and Computer Engineering, Carnegie Mellon University, Pittsburgh, PA

\begin{abstract}
This paper proposes microarchitecture-level models for Within Die (WID) process and system parameter variability that can be included in the design of high-performance processors. Since decisions taken at microarchitecture level have the largest impact on both performance and power, on one hand, and global variability effect, on the other hand, models and associated metrics are needed for their joint characterization and analysis. To assess how these variations affect or are affected by microarchitecture decisions, we propose a joint performance, power and variability metric that is able to distinguish among various design choices. As a design-driver for the modeling methodology, we consider a clustered high-performance processor implementation, along with its Globally Asynchronous, Locally Synchronous (GALS) counterpart. Results show that, when comparing the baseline, synchronous and its GALS counterpart, microarchitecturedriven impact of process variability translates into 2-10\% faster local clocks for the GALS case, while when taking into account the effect of on-chip temperature variability, local clocks can be $8-18 \%$ faster. If, in addition, voltage scaling (DVS) is employed, the GALS architecture with DVS is $26 \%$ better in terms of the joint quality metric employing energy, performance, and variability.
\end{abstract}

Keywords: variability, power consumption, GALS design

\section{REFERENCES}

[1] E. Acar, A. Devgan, R. Rao, Y. Liu, H. Su, S. Nassif, J. Burns, "Leakage and Leakage Sensitivity Computation for Combinational Circuits," in Proc. ACM/IEEE Intl. Symp. on Low Power Electronics and Design, pp. 96-99, Aug. 2003.

[2] A. Basu, S. Lin, V. Wason, A. Mehrotra, and K. Banerjee, "Simultaneous Optimization of Supply and Threshold Voltages for Low-Power and High-Performance Circuits in the Leakage Dominant Era," in Proc. ACM/IEEE Design Automation Conference, June 2004.

[3] S. Borkar, T. Karnik, V. De, "Design and Reliability Challenges in Nanometer Technologies," in Proc. ACM/IEEE Design Automation Conf., June 2004.

[4] K.A. Bowman, S.G. Duvall, J.M. Meindl, "Impact of Die-to-Die and WIthin-Die Parameter Fluctuations on the Maximum Clock Frequency Distribution for Gigascale Integration," in IEEE Journal of Solid-State Circuits, vol.37, no.2, Feb.2002.

[5] D. Brooks, V. Tiwari, and M. Martonosi, "Wattch: A Framework for Architectural-Level Power Analysis and Optimizations", in Proc. ACM Intl. Symp. on Computer Architecture, June 2000.

[6] J. A. Butts, and G. S. Sohi, "A Static Power Model for Architects," in Proc. Intl. Symp. on Microarchitecture, pp. 191-201, Dec. 2000.

[7] M. Eisele, J. Berthold, D. Schmidt-Landsiedel, R. Mahnkopf, "The Impact of Intra-Die Device Parameter Variations on Path Delays and on the Design for Yield of ow Voltage Digital Circuits," in Proc. ACM/IEEE Intl. Symp. on Low Power Electronics and Design, Aug. 1996.

[8] A. Iyer and D. Marculescu, "Power efficiency of Multiple Clock, Multiple Voltage Cores", in Proc. IEEE/ACM Intl. Conf. on Computer-Aided Design, San Jose, CA, Nov. 2002.

[9] S. McFarling, "Combining branch predictors", Technical Report DEC WRL Technical Note TN-36, DEC Western Research Laboratory, 1993.

[10] S. Palacharla, N. P. Jouppi, and J. E. Smith, "Complexity-effective superscalar processors," in Proc. ACM Intl. Symp. on Computer Architecture, June 1997.

[11] G. Semeraro, D.H. Albonesi, S.G. Dropsho, G. Magklis, S. Dwarkadas, and M.L. Scott, "Dynamic Frequency and Voltage Control for a Multiple Clock Domain Microarchitecture," in Proc. ACM Intl Symp. on Microarchitecture, Nov. 2002.

[12] K. Skadron, M. R. Stan, W. Huang, S. Velusamy, K. Sankaranarayanan, and D. Tarjan, "Temperature-Aware Microarchitecture," in Proc. ACM Intl. Symp. on Computer Architecture, June 2003.

[13] E. Talpes and D. Marculescu, "A Critical Analysis of Application-Adaptive Multiple Clock Processors," in Proc. ACM/IEEE Intl. Symp. on Low Power Electronics and Design, Aug. 2003. 
[14] J. Tschanz, J.T Kao, S.G. Narendra, R. Nair, D.A. Antoniadis, A.P. Chandrakasan, V. De, "Adaptive Body Bias for Reducing Impacts of Die-to-Die and Within-Die Parameter Variations on Microprocessor Frequency and Leakage," in IEEE Journal of Solid-State Circuits, vol.37, no.11, Nov. 2002. 


\title{
Energy-Efficient Physically Tagged Caches for Embedded Processors with Virtual Memory
}

\author{
Peter Petrov*, Daniel Tracy**, Alex Orailoglu** \\ *University of Maryland at College Park, ECE Department \\ **University of California at San Diego, CSE Department
}

\begin{abstract}
In this paper we present a low-power tag organization for physically tagged caches in embedded processors with virtual memory support. An exceedingly small subset of tag bits is identified for each application hot-spot so that only these tag bits are used for cache access with no perfromance sacrifice as they provide complete address resolution. The minimal subset of physical tag bits, i.e. the compressed tag, is dynamically updated following the changes in the physical address space of the application. Special support from the operating system (OS) is introduced in order to maintain the compressed tag during program execution. The compressed tag is updated by the OS to match the current set of physical memory pages allocated to the application. We have proposed efficient algorithms that are incorporated within the memory allocator and the dynamic linker in order to achieve dynamic update of the compressed tags in the cases where the mapping between virtual and physical addresses is modified; such cases include memory allocation/deallocation and swapping physical pages on the secondary memory storage. The only hardware support needed within the I/D-caches is the support for disabling bitlines of the tag arrays. An extensive set of experimental results demonstrates the efficacy of the proposed approach.
\end{abstract}

General Terms: Algorithms, Design, Experimentation, Performance

\section{REFERENCES}

[1] J. Montanaro et al., “A 160Mhz, 32b 0.5W CMOS RISC Microprocessor”, in IEEE ISCC, pp. 214-229, February 1996.

[2] J. Edmondson et al., "Internal organization of the Alpha 21164, a 300MHz 64-bit Quad-issue CMOS RISC Microprocessor", Digital Technical Journal, vol. 7, n. 1, pp. 119-135, 1995.

[3] A. Hasegawa et al, "Sh3: high code density, low power", in IEEE Micro, pp. 11-19, 1995.

[4] P. Petrov and A. Orailoglu, "Power Effficient Embedded Processor IP's through Application-Specific Tag Compression in Data Caches", in DATE, pp. 1065-1071, March 2002.

[5] M. Cekleov and M. Dubois, "Virtual-address caches. Part 1: problems and solutions in uniprocessors", IEEE Micro, vol. 17, n. 5, pp. 64-71, September 1997.

[6] J. Kim, S. Min, S. Jeon, B. Ahn, D. Jeong and C. Kim, "U-cache: a cost-effective solution to synonym problem", in $H P C A$, pp. 243-252, January 1995.

[7] R. Kessler, “The Alpha 21264 Microprocessor”, IEEE Micro, vol. 19, n. 1, pp. 24-36, March/April 1999.

[8] N. Bellas, I. Hajj and C. Polychronopoulos, "A detailed, transistor-level energy model for SRAM-based caches", in ISCAS, pp. 198.201, June 1999.

[9] T. Austin, E. Larson and D. Ernst, "SimpleScalar: An infrastructure for computer system modeling", IEEE Computer, vol. 35, n. 2, pp. 59-67, February 2002.

[10] C. Lee, M. Potkonjak and W. H. Mangione-Smith, "MediaBench: A Tool for Evaluating and Synthesizing Multimedia and Communications Systems", in 30th MICRO, pp. 330- 335, December 1997.

[11] E. Witchel and K. Asanovic, "The span cache: software controlled tag checks and cache line size", in Workshop on Complexity-Effective Design, 28th ISCA, June 2001. 


\title{
Hybrid Simulation for Embedded Software Energy Estimation
}

\author{
Anish Muttreja*, Anand Raghunathan**, Srivaths Ravi** and Niraj K. Jha* \\ *Dept. of Electrical Engineering, Princeton University, NJ 08544 \\ **NEC Labs, Princeton, NJ 08540
}

\begin{abstract}
Software energy estimation is a critical step in the design of energy-efficient embedded systems. Instruction-level simulation techniques, despite several advances, remain too slow for iterative use in system-level exploration. In this paper, we propose a methodology called hybrid simulation, which combines instruction set simulation with selective native execution (execution of some parts of the program directly on the simulation host computer), thereby overcoming the disadvantages of instruction-level simulation (low speed) and pure native execution (estimation accuracy, inapplicability to target-dependent code), while exploiting their advantages. Previously developed techniques for software energy macromodeling are utilized to estimate energy consumption for natively executed sub-programs. We identify and address the main challenges involved in hybrid simulation, and present an automatic tool flow for it, which analyzes a given program and selects functions for native execution in order to achieve maximum estimation efficiency while limiting estimation error. We have applied the proposed hybrid simulation methodology to a variety of embedded software programs, resulting in an average speed-up of $70 \%$ and estimation error of at most $6 \%$, compared to one of the fastest publicly-available instruction set simulators.
\end{abstract}

Keywords: Embedded Software, Energy Estimation, Energy Macromodels, Hybrid Simulation, Pointer Analysis

\section{REFERENCES}

[1] P. Magarshack and P. G. Paulin, "System-on-chip beyond the nanometer wall," in Proc. Design Automation Conf., June 2003, pp. 419-424.

[2] J. Zhu and D. D. Gajski, “A retargetable, ultra-fast instruction set simulator," in Proc. Design Automation \& Test Europe Conf., Mar. 1999, p. 62.

[3] A. Nohl, G. Braun, O. Schliebusch, R. Leupers, H. Meyr, and A. Hoffmann, "A universal technique for fast and flexible instruction-set architecture simulation," in Proc. Design Automation Conf., June 2002, pp. 22-27.

[4] W. S. Mong and J. Zhu, "Dynamosim: A trace-based dynamically compiled instruction set simulator," in Proc. Int. Conf. Computer-Aided Design, Nov. 2004, pp. 131-136.

[5] M. Reshadi, N. Bansal, P. Mishra, and N. Dutt, "An efficient retargetable framework for instruction-set simulation," in Proc. Int. Conf. Hardware/Software Codesign \& System Synthesis, Oct. 2003, pp. 13-18.

[6] W. Qin and S. Malik, "Flexible and formal modeling of microprocessors with application to retargetable simulation," in Proc. Design Automation \& Test Europe Conf., Mar. 2003, pp. 556-561.

[7] E. Perelman, G. Hamerly, and B. Calder, "Picking statistically valid and early simulation points," in Proc. Int. Conf. Parallel Architectures \& Compilation Techniques, 2003.

[8] V. S. P. Rapaka and D. Marculsecu, "Pre-charcterization free, efficient power/performance analysis of embedded and general purpose software applications," in Proc. Design Automation \& Test Europe Conf., 2003, pp. 504-509.

[9] T. K. Tan, A. Raghunathan, G. Lakshminarayana, and N. K. Jha, "High-level energy macro-modeling of embedded software," IEEE Trans. Computer-Aided Design, vol. 21, pp. 1037-1050, Sept. 2002.

[10] C. Brandolese, W. Fornaciari, F. Salice, and D. Sciuto, "Library functions timing characterization for sourcelevel analysis," in Proc. Design Automation \& Test Europe Conf., Mar. 2003, pp. 1132-1133.

[11] S. H. Yong and S. Horwitz, "Pointer-range analysis," in Proc. Int. Static Analysis Symp., Aug. 2004, pp. 133148.

[12] A. Muttreja, A. Raghunathan, S. Ravi, and N. K. Jha, "Automated energy/performance macromodeling of embedded software," in Proc. Design Automation Conf., June 2004, pp. 99-102.

[13] W. Qin, “The SimIt-ARM simulator.” [Online]. Available: http://www.ee.princeton.edu/»wqin/armsim.htm

[14] C. Lee, M. Potkonjak, and W. H. Mangione-Smith, "MediaBench: A tool for evaluating and synthesizing multimedia and communicatons systems," in Proc. Int. Symp. Microarchitecture, Nov. 1997, pp. 330-335. 
[15] M. R. Guthaus, J. S. Ringenberg, D. Ernst, T. M. Austin, T. Mudge, and R. B. Brown, "MiBench: A free, commercially representative embedded benchmark suite," in Proc. Wkshp. Workload Characterization, Dec. 2001, pp. 3-14.

[16] S. Patel, "Finger-print verification system." [Online]. Available: http://fvs.sourceforge.net/

[17] A. Sinha and A. P. Chandrakasan, "JouleTrack - A web based tool for software energy profiling," in Proc. Design Automation Conf., June 2001, pp. 220-225. 


\title{
Cooperative Multithreading on Embedded Multiprocessor Architectures Enables
} Energy-scalable Design

\author{
Patrick Schaumont ${ }^{1}$, Bo-Cheng Charles Lai ${ }^{1}$, Wei Qin ${ }^{2}$, Ingrid Verbauwhede ${ }^{1,3}$ \\ ${ }^{1}$ EE Department, UCLA, CA \\ ${ }^{2}$ ECE Department, Boston University, MA \\ ${ }^{3}$ ESAT, K.U. Leuven, BE
}

\begin{abstract}
We propose an embedded multiprocessor architecture and its associated thread-based programming model. Using a cycle-true simulation model of this architecture, we are able to estimate energy savings for a threaded $C$ program. The savings are obtained by voltage- and frequency-scaling of the individual processors. We port a fingerprint minutiae detection application onto this architecture, and show the resulting performance on single-, dual-, and quad-processor configurations. The energy-scaled quad-processor version results in a $77 \%$ energy reduction over the single-processor non-scaled implementation, at only a $2.2 \%$ degradation in cycle count.
\end{abstract}

General Terms: Design, Performance.

\section{REFERENCES}

[1] G. Andrews, "Concurrent programming - principles and practice", 102-105, Benjamin Cummings Publ. 1991.

[2] A. Andrei, M/ Schmitz, P. Eles, Z. Peng, B. Al-hashimi, "Overhead-Conscious Voltage Selection for Dynamic and Leakage Energy Reduction of Time-Constrained Systems," Proc. DATE 2004, 518 - 523.

[3] S. Eggers, J. Emer, H. Levy, J. Lo, R. Stamm, D. Tullsen, "Simultaneous multithreading: a platform for nextgeneration processors," IEEE Micro, 1997, 17(5):12-19.

[4] K. Flautner, S. Reinhardt, T. Mudge, "Automatic Performance Setting for Dynamic Voltage Scaling," Wireless networks, 2002, 8(5), 507-520.

[5] M. Forsell, "A Scalable high-performance computing solution for networks on chips,", IEEE Micro, 2002, 22(5):46-55.

[6] J. Goodacre, "Challenges in programming multiprocessor platforms," 4th International seminar on ApplicationSpecific MPSOC, France, 2004.

[7] J. Hennessy, D. Patterson, "Computer Architectures: A quantitative approach,” Ch. 6.3, MKP Publishers, 2002.

[8] A. Jerraya, W. Wolf, "Multiprocessor Systems-on-Chips," Morgan Kaufmann, Sept 2004, ISBN 0-12-385251-X.

[9] R. Jejurikar, C. Pereira, R. Gupta, "Leakage aware dynamic voltage scaling for real-time embedded systems," Proc. DAC 2004:275-280.

[10] J. Rabaey, "Power Management in Wireless SOCs," 4th International seminar on Application-Specific MPSOC, France, 2004.

[11] D. Keppel, "Tools and Techniques for Building Fast Portable Threads Packages," UWCSE 93-05-06, U. Washington, 1993.

[12] S. Martin, K. Flautner, T. Mudge, D. Blaauw, "Combined Dynamic Voltage Scaling and Adaptive Body Biasing for Lower Power Microprocessors under Dynamic Workloads," Proc. ICCAD 2002:721—725.

[13] F. Petrot, P. Gomez, "Lightweight implementation of the POSIX Threads API for an On-chip MIPS Multiprocessor with VCI Interconnect," Proc. 2003 DATE:51—56.

[14] J. Pouwelse, K. Langedoen, H. Sips, “Application-directed voltage scaling," IEEE Trans. on VLSI Systems, 11(5):812-826.

[15] S. Yang, K. Sakiyama, I. Verbauwhede, "A compact and efficient fingerprint verification system for secure embedded systems," $37^{\text {th }}$ Asilomar Conference, Nov 2003:2058-2062.

[16] C. Ussery, " Method of generating application specific integrated circuits using a programmable hardware architecture,” US. Pat. 6,075,935, 12/1/1997.

[17] W. Qin, S. Malik. Flexible and Formal Modeling of Microprocessors with Application to Retargetable Simulation, 2003 Design Automation and Test in Europe, March 2003, 556-561.

[18] P. Schaumont, I. Verbauwhede, "Interactive cosimulation with partial evaluation," 2004 Design Automation and Test in Europe, February 2004, 642-647. 


\begin{abstract}
Minimizing power consumption is one of the most important objectives in IC design. Resizing gates and assigning different $\mathrm{V}_{\mathrm{t}}$ 's are common ways to meet power and timing budgets. We propose an automatic implementation of both these techniques using a mixed-integer linear programming model called MLP-exact, which minimizes a circuit's total active-mode power consumption. Unlike previous linear programming methods which only consider local optimality, $M L P$-exact can find a true global optimum. An efficient, non-optimal way to solve the MLP model, called MLP-fast, is also described. We present a set of benchmark experiments which show that MLP-fast is much faster than MLPexact, while obtaining designs with only slightly higher power consumption. Furthermore, the designs generated by MLP-fast consume $30 \%$ less power than those obtained by conventional, sensitivity-based methods.
\end{abstract}

Keywords: Low power, linear programming, dual $\mathrm{V}_{\mathrm{t}}$, gate sizing

\title{
REFERENCES
}

[1] S. Augsburger et al., "Reducing Power with Dual Supply, Dual Threshold and Transistor Sizing", Proc. ICCD, 2002.

[2] M. R. C. M. Berkelaar et al., "Gate Sizing in MOS Digital Circuits with Linear Programming", Proc. DATE, 1990.

[3] Berkeley Predictive Technology Model, http://wwwdevice.eecs.berkeley.edu/ ptm/.

[4] R. Burch et al., "A Monte Carlo Approach for Power Estimation", IEEE Trans. on VLSI, 1993.

[5] A. Chatterjee et al., "An Investigation of the Impact of Technology Scaling on Power Wasted as Short-Circuit Current in Low Voltage Static CMOS", Proc. ISLPED, 1996.

[6] J. P. Fishburn, and A. E. Dunlop, “TILOS: A Posynomial Programming Approach to Transistor Sizing”, Proc. ICCAD, 1985.

[7] F. Gao and J. P. Hayes, "Gate Sizing and Vt Assignment for Active-Mode Leakage Power Reduction", Proc. ICCD, 2004.

[8] A. Ghosh et al., "Estimation of Average Switching Activity in Combinational and Sequential Circuits", Proc. $D A C, 1992$.

[9] R. Ho et al., "The Future of Wires", IEEE Proceedings, 2001.

[10] W. Hung et al., "Total Power Optimization through Simultaneously Multiple-Vdd Multiple-Vth Assignment and Device Sizing with Stack Forcing", Proc. ISLPED, 2004.

[11] ILOG cplex. http://www.ilog.com/products/cplex/.

[12] D. Nguyen et al., "Minimization of Dynamic and Static Power Through Joint Assignment of Threshold Voltages and Sizing Optimization", Proc. ISLPED, 2003.

[13] P. Pant et al., "Dual-threshold Voltage Assignment with Transistor Sizing for Low Power CMOS Circuits", IEEE Trans. On VLSI, 2001.

[14] T. Pering, T. Burd, and R. Broderson, "Voltage Scheduling in the IpARM Micorprocessor System", Proc. ISLPED, 2000.

[15] A. Srivastava et al., "Concurrent Sizing, Vdd and Vth Assignment for Low-Power Design”, Proc. DATE, 2004.

[16] N. H. E. Weste and K. Eshraghian. Principles of CMOS VLSI Design: A Systems Perspective, Addison-Wesley, 1993. 


\title{
An Effective Power Mode Transition Technique in MTCMOS Circuits
}

\author{
Afshin Abdollahi*, Farzan Fallah**, Massoud Pedram* \\ *University of Southern California \\ **Fujitsu Labs. of America
}

\begin{abstract}
The large magnitude of supply/ground bounces, which arise from power mode transitions in power gating structures, may cause spurious transitions in a circuit. This can result in wrong values being latched in the circuit registers. We propose a design methodology for limiting the maximum value of the supply/ground currents to a user-specified threshold level while minimizing the wake up (sleep to active mode transition) time. In addition to controlling the sudden discharge of the accumulated charge in the intermediate nodes of the circuit through the sleep transistors during the wake up transition, we can eliminate short circuit current and spurious switching activity during this time. This is in turn achieved by reducing the amount of charge that must be removed from the intermediate nodes of the circuit and by turning on different parts of the circuit in a way that causes a uniform distribution of current over the wake up time. Simulation results show that, compared to existing wakeup scheduling methods, the proposed techniques result in a one to two orders of magnitude improvement in the product of the maximum ground current and the wake up time.
\end{abstract}

General Terms: Algorithms, Performance, Design, Reliability.

\section{References}

[1] J. Kao, A. Chandrakasan and D. Antoniadis, "Transistor Sizing Issues and Tool for Multi-Threshold CMOS Technology," Design Automation Conf., pp. 409-414, 1997.

[2] J. Kao, S. Narenda and A. Chandrakasan, "MTCMOS Hierarchical Sizing Based on Mutual Exclusive Discharge Patterns," Design Automation Conf., pp. 495 - 500, 1998.

[3] M.Anis, S. Areibi, M. Mahmoud and M. Elmasry, "Dynamic and Leakage Power Reduction in MTCMOS Circuits Using an Automated Efficient Gate Clustering Technique," Design Automation Conf., pp. 480-485, 2002.

[4] S. Kim, S.V. Kosonocky, D. R. Knebel, and K. Stawiasz, "Experimental measurement of a novel power gating structure with intermediate power saving mode," Intl. Symp. on Low Power Electronics and Design, pp. 20-25, 2004.

[5] S. Kim, S. V. Kosonocky, Stephen, and D. R. Knebel, "Understanding and minimizing ground bounce during mode transition of power gating structures", Intl. Symp. on Low Power Electronics and Design, pp. 22-25, 2003.

[6] Hyo-Sig Won, et al., "An MTCMOS Design Methodology and Its Application to Mobile Computing," Intl. Symp. on Low Power Electronics and Design, pp. 110-115, 2003.

[7] Usami, et al., "Automated Selective Multi-Threshold Design for Ultra-Low Standby Applications," Intl. Symp. on Low Power Electronics and Design, pp. 202-206, 2002.

[8] M. Johnson, D. Somasekhar, and K. Roy, "Leakage Control with Efficient use of Transistor Stacks in Single Threshold CMOS," Design Automation Conf., pp. 442-445, 1999.

[9] T. Cormen, C. Leiserson, R. Rivest, and C. Stein, Introduction to Algorithms, 2nd ed. Cambridge, MA: MIT Press, 2001. 


\title{
A Self-adjusting Scheme to Determine the Optimum RBB by Monitoring Leakage Currents
}

\author{
Nikhil Jayakumar*, Sandeep Dhar**, Sunil P Khatri* \\ * Department of EE, Texas A\&M University, College Station TX 77843 \\ ** National Semiconductor, Longmont, CO 80501
}

\begin{abstract}
Reverse body biasing (RBB) is often used to reduce the leakage power of a device. However, recent research has shown that if this applied RBB is too high, the leakage power can actually increase due to the contribution of Band-to-Band Tunneling (BTBT) currents. Hence, there exists an optimal RBB value at which the leakage is minimum. This optimum point can vary with temperature and process variations. In this paper we show that it is desirable to operate at the optimal RBB point which minimizes total leakage. We present a scheme that monitors the total leakage current (the sum of the sub-threshold, BTBT and gate leakage) of an IC with a representative leaking device and, using this monitored value, automatically finds the optimum RBB value across temperature and process corners, using a self-adjusting circuit. Our approach has a modest placed-and-routed area utilization, and a low power consumption.
\end{abstract}

Keywords: Leakage power, Body-biasing, Self-adjusting

\section{REFERENCES}

[1] "The International Technology Roadmap for Semiconductors." http://public.itrs.net/, 2003.

[2] A. Keshavarzi, S. Narendra, S. Borkar, C. Hawkins, K. Royi, and V. De, "Technology scaling behavior of optimum reverse body bias for standby leakage power reduction in CMOS ICs," in Proceedings International Symposium on Low Power Electronics and Design, pp. 252-254, Aug 1999.

[3] C. Neau. Personal communication, June 2004.

[4] C. Neau and K. Roy, "Optimal body bias selection for leakage improvement and process compensation over different technology generations," in Proceedings of the International Symposium on Low Power Electronics and Design, pp. 116 - 121, Aug 2003.

[5] X. Liu and S. Mourad, "Performance of submicron CMOS devices and gates with substrate biasing," in The IEEE International Symposium on Circuits and Systems, vol. 4, (Geneva), pp. 9-12, May 2000.

[6] Y.-S. Lin, C.-C. Wu, C.-S. Chang, R.-P. Yang, W.-M. Chen, J.-J. Liaw, and C. Diaz, "Leakage scaling in deep submicron CMOS for SoC," IEEE Transactions on Electron Devices, vol. 49, pp. 1034-1041, June 2002.

[7] S. Mukhopadhyay, H. Mahmoodi-Meimand, C. Neau, and K. Roy, "Leakage in nanometer scale CMOS circuits," in International Symposium on VLSI Technology, Systems, and Applications, pp. 307-312, 2003.

[8] T. Kuroda, T. Fujita, S. Mita, T. Nagamatsu, S. Yoshioka, K. Suzuki, F. Sano, M. Norishima, M. Murota, M. Kako, M. K. M. Kakumu, and T. Sakurai, "A 0.9-v, 150-mhz, 10-mw, 4 mm 2, 2-d discrete cosine transform core processor with variable threshold-voltage (VT) scheme," IEEE Journal of Solid-State Circuits, vol. 31, pp. 17701779, Nov 1996.

[9] T. Kobayashi and T. Sakurai, "Self-adjusting threshold-voltage scheme (SATS) for low-voltage high-speed operation," in Proceedings of the IEEE Custom Integrated Circuits Conference, pp. 271-274, May 1994.

[10] H. Soeleman, K. Roy, and B. Paul, "Robust subthreshold logic for ultra-low power operation," IEEE Transactions on Very Large Scale Integration (VLSI) Systems, vol. 9, no. 1, pp. 90-99, 2001. 
DAC05, pages 47-50

\title{
Enhanced Leakage Reduction Technique by Gate Replacement
}

Lin Yuan and Gang Qu

Electrical and Computer Engineering Department and Institute for Advanced Computer Studies, University of Maryland, College Park, MD, 20742 USA

\begin{abstract}
Input vector control (IVC) technique utilizes the stack effect in CMOS circuit to apply the minimum leakage vector (MLV) to the circuit at the sleep mode to reduce leakage. Additional logic gates can be inserted as control points to make it more effective. In this paper, we propose a gate replacement technique that further enhances the leakage reduction. The basic idea is to replace a gate that is in its worst leakage state by another library gate while keeping the circuit's correct functionality at the active mode. We also develop a divide-and-conquer approach that integrates a fast gate replacement heuristic, an optimal MLV search strategy for tree circuit, and a genetic algorithm to connect the tree circuits. We conduct experiments on the MCNC91 benchmark circuits. The results reveal that our technique can reduce additional $10 \%$ to $24 \%$ leakage over the best known IVC methods and the optimal MLV with no delay penalty and little area increase.
\end{abstract}

Keywords: Leakage reduction, gate replacement, MLV

\section{REFERENCES}

[1] A. Abdollahi, F. Fallah, and M. Pedram, "Leakage Current Reduction in CMOS VLSI Circuits by Input Vector Control", IEEE Trans. on VLSI, Vol. 12, pp. 140-154, Feb. 2004.

[2] F. Aloul, S. Hassoun, K. Sakallah, D. Blaauw, "Robust SAT-Based Search Algorithm for Leakage Power Reduction", International Workshop on Integrated Circuit Design, pp. 167-177, 2002.

[3] B.H. Calhoun, F.A. Honore, and A. Chandrakasan, "Design Methodology for Fine-Grained Leakage Control in MTCMOS”, ISLPED, pp. 104-109, 2003.

[4] D. Duarte, Y. Tsai, N. Vijaykrishnan, and M. Irwin, "Evaluating Run-Time Techniques for Leakage Power Reduction", IEEE International Conference on VLSI Design, pp. 31-38, 2002.

[5] F. Gao and J.P. Hayes, "Exact and Heuristic Approaches to Input Vector Control for Leakage Power Reduction", ICCAD, pp. 527-532, 2004.

[6] J. Halter, and F. Najm, "A Gate-Level Leakage Power Reduction Method for Ultra Low Power CMOS Circuits", CICC, pp 475-478, 1997.

[7] M.C. Johnson, D. Somasekhar, and K. Roy, "Models and Algorithms for Bounds on Leakage in CMOS Circuits", IEEE Trans. on CAD, Vol. 18, pp. 714-725, 1999.

[8] J. Kao, S. Narendra, A. Chandrakasan, "Subthreshold Leakage Modeling and Reduction Techniques", ICCAD, pp. 141-148, 2002

[9] R.M. Rao, F. Liu, J.L. Burns, and R.B. Brown, "A Heuristic to Determine Low Leakage Sleep State Vectors for CMOS Combinational Circuits", ICCAD, pp. 689-692, 2003. 


\title{
Automated Nonlinear Macromodelling of Output Buffers for High-Speed Digital Applications
}

\author{
Ning Dong, Jaijeet Roychowdhury \\ Department of Electrical and Computer Engineering, University of Minnesota
}

\begin{abstract}
We present applications of a recently developed automated nonlinear macromodelling approach to the important problem of macromodelling high-speed output buffers/drivers. Good nonlinear macromodels of such drivers are essential for fast signal-integrity and timing analysis in highspeed digital design. Unlike traditional black-box modelling techniques, our approach extracts nonlinear macromodels of digital drivers automatically from SPICE-level descriptions. Thus it can naturally capture transistor-level nonlinearities in the macromodels, resulting in far more accurate signal integrity analysis, while retaining significant speedups. We demonstrate the technique by automatically extracting macromodels for two typical digital drivers. Using the macromodel, we obtain about $8 \times$ speedup in average with excellent accuracy in capturing different loading effects, crosstalk, simultaneous switching noise (SSN), etc. .

Keywords: nonlinear macromodeling, I/O buffer macromodeling
\end{abstract}

\section{REFERENCES}

[1] I/O Buffer Information Specification (IBIS) Ver. 4.1. http://www.eigroup.org/ibis.

[2] A. Boni, A. Pierazzi, and D. Vecchi. LVDS I/O Interface for Gb/s-per-Pin Operation in 0.35-um CMOS. IEEE J. Solid-State Ckts., 36(4):706-711, Apr. 2001.

[3] N. Dong and J. Roychowdhury. Piecewise Polynomial Nonlinear Model Reduction. Proc. IEEE DAC, 2003.

[4] N. Dong and J. Roychowdhury. Automated Extraction of Broadly Applicable Nonlinear Analog Macromodels from SPICE-level Descriptions. Proc. IEEE CICC, 2004.

[5] P. Feldmann and R. Freund. Efficient linear circuit analysis by Pad'e approximation via the Lanczos process. IEEE Trans. CAD, 14(5):639-649, May 1995.

[6] R. Freund. Reduced-order modeling techniques based on Krylov subspaces and their use in circuit simulation. Technical Report 11273-980217-02TM, Bell Laboratories, 1998.

[7] R. W. Freund. Krylov-subspace methods for reduced-order modeling in circuit simulation. Journal of Computational and Applied Mathematics, 123:395-421, 2000.

[8] K. Gallivan, E. Grimme, and P. V. Dooren. Asymptotic waveform evaluation via a lanczos method. Appl. Math. Lett., 7:75-80, 1994.

[9] E. Grimme. Krylov Projection Methods for Model Reduction. PhD thesis, University of Illinois, EE Dept, Urbana-Champaign, 1997

[10] P. Heydari. Design and analysis of low-voltage current-mode logic buffers. Fourth International Symposium on Quality Electronic Design, pages 293-298, 2003.

[11] P. Li and L. T. Pileggi. NORM: Compact Model Order Reduction of Weakly Nonlinear Systems. Proc. IEEE $D A C, 2003$.

[12] B. Mutnury, M. Swaminathan, and J. Libous. Macro-modeling of non-linear I/O drivers using spline functions and finite time difference approximation. Proc. Electrical Performance of Electronic Packaging, pages 273-276, 2003.

[13] J. Phillips. Projection frameworks for model reduction of weakly nonlinear systems. In Proc. IEEE DAC, June 2000.

[14] M. Rewienski and J. White. A Trajectory Piecewise-Linear Approach to Model Order Reduction and Fast Simulation of Nonlinear Circuits and Micromachined Devices. IEEE Trans. CAD, 22(2), Feb. 2003.

[15] J. Roychowdhury. Reduced-order modelling of linear time-varying systems. In Proc. ICCAD, Nov. 1998.

[16] J. Roychowdhury. Reduced-order modelling of time-varying systems. IEEE Trans. Ckts. Syst. - II: Sig. Proc., 46(10), Nov. 1999.

[17] J. Roychowdhury. An Overview of Automated Macromodelling Techniques for Mixed-Signal Systems. Proc. IEEE CICC, 2004.

[18] J. Savoj and B. Razavi. A 10-Gb/s CMOS clock and data recovery circuit with a half-rate linear phase detector. IEEE J. Solid-State Ckts., 36:761-768, May 2001. 
[19] I. Stievano, F. Canavero, Z. Chen, G. katopis, and I. Maio. Parametric macromodels of drivers for SSN simulations. IEEE Trans. Electromagnetic Compat., 2:616-621, Aug. 2003.

[20] I. Stievano, I. Maio, and F. Canavero. Parametric Macromodels of Digital I/O Ports. IEEE trans. on advanced packaging, 25(2):255-264, May 2002.

[21] D. Vasilyev, M. Rewienski, and J. White. A TBR-based Trajectory Piecewise-Linear Algorithm for Generating Accurate Low-order Models for Nonlinear Analog Circuits and MEMS. Proc. IEEE DAC, 2003.

[22] B. Yang and B. MacGaughy. An Essentially Non-Oscillatory (ENO) High-Order Accurate Adaptive Table Model for Device Modeling. Proc. IEEE DAC, 2004.

[23] Q. Zhang and K. Gupta. Neural Networks for RF and Microwave Design. Boston: Artech House, 2000. 


\title{
Systematic Development of Analog Circuit Structural Macromodels through Behavioral Model Decoupling
}

\author{
Ying Wei, Alex Doboli
}

Department of Electrical and Computer Engineering, Stony Brook University, Stony Brook, NY

\begin{abstract}
This paper presents a systematic methodology to create customized structural macromodels for a specific analog circuit. The novel contributions of the method include definition of the building block behavioral concept and two original algorithms to generate structural models. Experiments are offered for two-stage opamp and operational transconductor amplifier (OTA) circuits. The automatically produced models are accurate, offer design insight, and require low modeling effort.
\end{abstract}

Keywords: Analog circuits, Structural macromodel

\section{REFERENCES}

[1] G. Gielen, R. Rutenbar, "Computer Aided Design of Analog and Mixed-signal Integrated Circuits", Proc. Of IEEE, pp. 1825-1851, No. 12, 2000.

[2] IEEE Trans. CAD, Special Issue on Modeling and Simulation, Apr. 2003.

[3] X. Huang et al,"Modeling Nonlinear Dynamics in Analog Circuits via Root Localization", IEEE Trans. CAD, pp. 895-907, Jul. 2003.

[4] G. J. Gomez et al,“A Generic Parameterizable CMOS OTA Macromodel”, IEEE Trans. C\&S, Feb. 1995.

[5] B. Razavi, "Design of Analog CMOS Integrated Circuits", McGraw-Hill, 2002.

[6] P. Dobrovolny et al, "Analysis and Compact Behavioral Modeling of Nonlinear Distortion in Analog Communication Circuits",IEEE Trans. CAD, Sept. 2003.

[7] E. O. Guerra et al,"A Hierarchical Approach for the Symbolic Analysis of Large Analog Integrated Circuits", Proc. DATE, pp.48-52, 2000.

[8] A. Doboli, R.Vemuri, "A Regularity-based Hierarchical Symbolic Analysis Method for Large-scale Analog Networks”, IEEE Trans. C\&S-II, pp. 1054-1067, Nov. 2001.

[9] F. Leyn et al,“Analog Small-Signal Modeling I: Behavioral Signal Path Modeling for Analog Integrated Circuits”,IEEE Trans. C\&S-II, pp. 701-711, Jul. 2001.

[10] F. Leyn et al,“Analog Small-Signal Modeling II: Behavioral Signal Path Modeling for Analog IC",IEEE Trans. C\&S-II, pp. 712-721, Jul. 2001.

[11] H. Zhang, A. Doboli,"Fast Time-Domain Symbolic Simulation for Synthesis of $\Delta-\Sigma$ Analog-Digital Converters", Proc. ISCAS, pp. 125-128, 2004.

[12] W. Daems et al, "Simulation-Based Generation of Posynomial Performance Models for the Sizing of Analog Integrated Circuits", IEEE Trans. CAD, May 2003.

[13] C. Motchenbacher, J. Connelly,"Low Noise Electronic System Design”, Wiley, 2000.

[14] Y. Zhang et al, "A 1.8v Continuous-Time $\Delta^{-} \Sigma$ Modulator with 2.5MHz Bandwidth", Proc. MWSCAS, pp. 140-143, 2002. 


\title{
A Combined Feasibility and Performance Macromodel for Analog Circuits
}

\author{
Mengmeng Ding, Ranga Vemuri \\ ECECS Department, University of Cincinnati, Cincinnati, OH, USA
}

\begin{abstract}
The need to reuse the performance macromodels of an analog circuit topology challenges existing regression based modeling techniques. A model of good reusability should have a number of independent design parameters and each parameter can vary in a large numeric range. On the other hand, these requirements can cause a large percentage of functionally incorrect designs in the design space and thus results in a sparse feasible design space. They also complicate the mathematical relationship between the performance parameters and the design parameters. In order to tackle these challenges, this paper presents a combined feasibility and performance macromodel based on Support Vector Machines (SVMs). The feasibility model identifies the feasible designs that satisfy the design constraints. The performance macromodel is valid for feasible designs. Feasibility macromodeling is formulated as a classification problem while performance macromeling as a regression problem. An active learning scheme [5] has been applied to improve the accuracy of the feasibility model much faster than only using uniformly distributed designs in the entire design space. Our experiment shows that the performance macromodels in the feasible design space are more accurate and faster to construct and evaluate than performance macromodels in the entire design space without functional or performance constraints considered.
\end{abstract}

Keywords: Performance macromodeling, feasibility models, active learning

\section{REFERENCES}

[1] O. Bajdechi, J. H. Huijsing, and G. Gielen. Optimal design of delta-sigma ADCs by design space exploration. In Proceedings of the 39th conference on Design automation, pages 443-448. ACM Press, 2002.

[2] C.-C. Chang and C.-J. Lin. LIBSVM: a library for support vector machines, 2001. Software available at http://www.csie.ntu.edu.tw/ cjlin/libsvm.

[3] W. Daems, G. Gielen, and W. Sansen. Simulation-based generation of posynomial performance models for the sizing of analog integrated circuits. IEEE Transactions on Computer-Aided Design of Integrated Circuits and Systems, 22(5):517-534, 2003.

[4] B. De Smedt and G. Gielen. WATSON: design space boundary exploration and model generation for analog and RFIC design. IEEE Transactions on Computer-Aided Design of Integrated Circuits and Systems, 22(2):213-224, 2003.

[5] M. Ding and R. Vemuri. An active learning scheme using support vector machines for analog circuit feasibility classification. In Proceedings of the 18th international conference on VLSI design, 2005.

[6] A. Doboli, A. Nunez-Aldana, N. Dhanwada, S. Ganesan, and R. Vemuri. Behavioral synthesis of analog systems using two-layered design space exploration. In Proceedings of the 36th ACM/IEEE conference on Design automation, pages 951-957. ACM Press, 1999.

[7] M. Hershenson, S. Boyd, and T. Lee. Optimal design of a CMOS op-amp via geometric programming. IEEE Transactions on Computer-Aided Design of Integrated Circuits and Systems, 20(1):1-21, 2001.

[8] T. Kiely and G. Gielen. Performance modeling of analog integrated circuits using least-squares support vector machines. In Proceedings of Design, Automation and Test in Europe Conference and Exhibition, pages 448-453, 2004.

[9] A. Nunez-Aldana and R. Vemuri. An analog performance estimator for improving the effectiveness of CMOS analog systems circuit synthesis. In Proceedings of Design, Automation and Test in Europe Conference and Exhibition, pages 406-411, 2001.

[10] G. Stehr, M. Pronath, F. Schenkel, H. Graeb, and K. Antreich. Initial sizing of analog integrated circuits by centering within topology-given implicit specifications. In International Conference on Computer Aided Design, pages $241-246,2003$.

[11] V. Vapnik. The nature of statistical learning theory. Springer, New York, 1995. 
[12] M. Vogels and G. Gielen. Architectural selection of A/D converters. In Proceedings of the 40th conference on Design automation, pages 974-977. ACM Press, 2003.

[13] G. Wolfe and R. Vemuri. Extraction and use of neural network models in automated synthesis of operational amplifiers. IEEE Transactions on Computer-Aided Design of Integrated Circuits and Systems, 22(2):198-212, 2003.

[14] S. Zizala, J. Eckmueller, and H. Grab. Fast calculation of analog circuits' feasibility regions by low level functional measures. In IEEE International Conference on Electronics, Circuits and Systems, pages 85-88, 1998. 


\section{ESL: Tales from the Trenches}

Chair: $\quad$ David Maliniak - Electronic Design, Paramus, NJ

Panelists: $\quad$ Terry Doherty - Emulex, Bothell, WA

Peter McShane - Northrop Grumman, Redondo Beach, California

Suhas A. Pai- Qualcomm, San Diego, CA

Sriram Sundararajan - Texas Instruments Inc., Dallas, TX

Dr. Soo-Kwan Eo - ST Microelectronics, SEOUL, Korea

Pascal Urard - ST Microelectronics, The Netherlands

\section{PANEL SUMMARY}

Electronic System-Level design has arrived - but can ESL provide the bridge from systems to silicon? Comprised of real world designers, this DAC ESL panel will examine and debate what works, what doesn't, and what the gaps are in the methodology and tool offerings. Panelists from a variety of industry segments, including Military/aerospace, storage area networks (SAN), wireless communications and consumer electronics, will share their experiences, lessons learned and further needs.

Does ESL bridge the gap between systems to silicon? Hear from designers about their real world experience with ESL. What worked according to expectations? What didn't? What are the gaps in the methodology and tool offerings that need to be filled, and why?

This panel of ESL design methodology users will give us a "reality check" that will enable potential users to make an adoption decision, and enable ESL design tool suppliers to evaluate their product strategies against "big picture" requirements.

Panelists will address primary areas of concern, such as:

Methodology Usage: What do you use ESL design for? Is it for algorithm development alone? Are you using it for hardware/software partitioning? Have you used it for embedded system architecture development for performance optimization and/or for SoC platform development? Are you using ESL for embedded software development, using the system architecture model as a development platform? Are you doing any highlevel synthesis of RTL? Did ESL help you with your system testbench development or HW/SW co-verification?

Industry-Level Initiatives: How has language standardization, such as SystemC, impacted your design efforts? What other industry-level initiatives would be of use - standard TLM methodology, or other?

Tools: Do you use commercial tools or open source software? What was your selection criteria? Do you use domain-specific tools for algorithm development and implementation? Did you develop your own tools - if so, why? Do your proprietary tools have specific attributes that you think can be incorporated into commercial tools? How do ESL tools compare with your original expectations?

ROI: What was your overall payback in terms of time, effort and money consumption, reusability, risk management and overall success? How do these compare with your original expectations?

Keywords: Electronic system-level design 


\title{
Parameterized Block-Based Statistical Timing Analysis with Non-Gaussian Parameters, Nonlinear Delay Functions
}

\author{
Hongliang Chang ${ }^{1}$, Chandu Visweswariah ${ }^{2}$, Sambasivan Narayan $^{3}$, Vladimir Zolotov ${ }^{2}$ \\ ${ }^{1}$ Dept. of CSE, University of Minnesota \\ ${ }^{2}$ IBM T.J.W. Research Center, Yorktown Heights, NY \\ ${ }^{3}$ IBM Systems \& Technology, Essex Junction, VT
}

\begin{abstract}
Variability of process parameters makes prediction of digital circuit timing characteristics an important and challenging problem in modern chip design. Recently, statistical static timing analysis (statistical STA) has been proposed as a solution. Unfortunately, the existing approaches either do not consider explicit gate delay dependence on process parameters [3] - [6] or restrict analysis to linear Gaussian parameters only [1, [2]. Here we extend the capabilities of parameterized block-based statistical STA [1] to handle nonlinear function of delays and nonGaussian parameters, while retaining maximum efficiency of processing linear Gaussian parameters. Our novel technique improves accuracy in predicting circuit timing characteristics and retains such benefits of parameterized block-based statistical STA as an incremental mode of operation, computation of criticality probabilities and sensitivities to process parameter variations. We implemented our technique in an industrial statistical timing analysis tool. Our experiments with large digital blocks showed both efficiency and accuracy of the proposed technique.
\end{abstract}

General Terms: Algorithms, performance, design.

\section{REFERENCES}

[1] C. Visweswariah, K. Ravindran, K. Kalafala, S. G. Walker and S. Narayan, "First-Order Incremental BlockBased Statistical Timing Analysis", DAC 2004, pp. 331-336.

[2] H. Chang and S. Sapatnekar, "Statistical timing analysis considering spatial correlation in a PERT-like traversal," ICCAD 2003, pp. 621-625.

[3] J. J. Liou, K. T. Cheng, S. Kundu and A. Krstic, "Fast statistical timing analysis by probabilistic event propagation," DAC 2001, pp. 661-666.

[4] M. Orshansky and K. Keutzer, "A general probabilistic framework for worst case timing analysis," DAC 2002, pp. 556-561.

[5] A. Devgan and C. Kashyap, "Block-based static timing analysis with uncertainty," ICCAD 2003, pp. 607-614.

[6] A. Agarwal, V. Zolotov and D. Blaauw, "Statistical timing analysis using bounds and selective enumeration," IEEE Transactions on CAD of Integrated Circuits and Systems, vol. 22, no. 9, Sept 2003, pp.1243-1260.

[7] S. Bhardwaj, S.B.K. Vrudhula and D. Blaauw, "TAU: Timing Analysis Under Uncertainty", ICCAD 2003, pp. 615-620

[8] C.E. Clark, "The Greatest of a Finite Set of Random Variables", Operations Research, vol. 9, 1961, pp. 85-91.

[9] X. Li, J. Le, P. Gopalakrishnan and L. Pileggi, "Asymptotic probability extraction for non-Normal distributions of circuit performance", ICCAD 2004, pp. 1-9. 


\author{
Yaping Zhan*, Andrzej J. Strojwas*, Xin Li*, Lawrence T. Pileggi*, \\ David Newmark**, Mahesh Sharma** \\ *Department of ECE, Carnegie Mellon University, Pittsburgh, PA, \\ **Advanced Micro Devices Inc., Austin, TX
}

\begin{abstract}
Process variations have a growing impact on circuit performance for today's integrated circuit (IC) technologies. The Non-Gaussian delay distributions as well as the correlations among delays make statistical timing analysis more challenging than ever. In this paper, we present an efficient block-based statistical timing analysis approach with linear complexity with respect to the circuit size, which can accurately predict Non-Gaussian delay distributions from realistic nonlinear gate and interconnect delay models. This approach accounts for all correlations, from manufacturing process dependence, to re-convergent circuit paths to produce more accurate statistical timing predictions. With this approach, circuit designers can have increased confidence in the variation estimates, at a low additional computation cost.
\end{abstract}

Keywords: Statistical timing, process variation

\title{
REFERENCES
}

[1] M. Orshansky and K. Keutzer, "A General Probabilistic Framework for Worst Case Timing Analysis", Proc. DAC, pp 556-561, June 2002

[2] J. A. G. Jess and K. Kalafala et al, "Statistical timing for parametric yield prediction of digital integrated circuits", Proc. DAC, pp. 932-937, June 2003

[3] A. Devgan and C. Kashyap, "Block-based Static Timing Analysis with Uncertainty", IEEE ICCAD, pp. 607614, November 2003

[4] A. Agarwal, D. Blaauw, V. Zolotov And S. B. K. Vrudhula, "Statistical Timing Analysis with Uncertainty", DATE, pp. 62 - 67, 2003

[5] H. Chang, S. S. Sapatnekar, "Statistical timing analysis considering spatial correlations using a single PERT-like traversal", IEEE ICCAD, pp. 621-625 November 2003

[6] C. Visweswariah, K. Ravindran, K. Kalafala, S. G. Walker, S. Narayan, "First-Order Incremental Block-Based Statistical Timing Analysis", Proc. 2004 DAC, pp. 331-336, June 2004

[7] J. Le, X. Li, L. T. Pileggi, "STAC: Statistical Timing Analysis with Correlation", Proc. DAC, pp. 343-348, June 2004

[8] D. F. Morrison, "Multivariate Statistical Methods", New York: McGraw-Hill, 1976

[9] S. R. Nassif, "Modeling and Analysis of Manufacturing Variations", IEEE CICC, pp. 223-228, 2001

[10] X. Li, J. Le, P. Gopalakrishnan, and L. T. Pileggi, "Asymptotic Probability Extraction for Non-Normal Distributions of Circuit Performance", IEEE ICCAD, pp. 2-9, November 2004. 
DAC05, pages 83-88

\title{
Correlation-Preserved Non-Gaussian Statistical Timing Analysis with Quadratic Timing Model
}

\author{
Lizheng Zhang, Weijen Chen, Yuhen Hu, John A. Gubner, Charlie Chung-Ping Chen \\ ECE Department,University of Wisconsin, Madison, WI, USA
}

\begin{abstract}
Recent study shows that the existing first order canonical timing model is not sufficient to represent the dependency of the gate delay on the variation sources when processing and operational variations become more and more significant. Due to the nonlinearity of the mapping from variation sources to the gate/wire delay, the distribution of the delay is no longer Gaussian even if the variation sources are normally distributed. A novel quadratic timing model is proposed to capture the non-linearity of the dependency of gate/wire delays and arrival times on the variation sources. Systematic methodology is also developed to evaluate the correlation and distribution of the quadratic timing model. Based on these, a novel statistical timing analysis algorithm is propose which retains the complete correlation information during timing analysis and has the same computation complexity as the algorithm based on the canonical timing model. Tested on the ISCAS circuits, the proposed algorithm shows $10 \times$ accuracy improvement over the existing first order algorithm while no significant extra runtime is needed.
\end{abstract}

General Terms: Algorithms, Performance, Verification

\section{REFERENCES}

[1] J.-J. Liou, A. Krstic, L.-C. Wang, and K.-T. Cheng, "False-path-aware statistical timing analysis and efficient path selection for delay testing and timing validation," Design Automation Conference, 2002. Proceedings. 39th, pp. 566 - 569, June 2002.

[2] M. Orshansky, "Fast computation of circuit delay probability distribution for timing graphs with arbitary node correlation," TAU'04, Feb 2004.

[3] M. Orshansky and K. Keutzer, "A general probabilistic framework for worst case timing analysis," Design Automation Conference, 2002. Proceedings. 39th, pp. 556 - 561, June 2002.

[4] A. Agarwal, D. Blaauw, V. Zolotov, S. Sundareswaran, M. Zhao, K. Gala, and R. Panda, "Statistical delay computation considering spatial correlations," Design Automation Conference, 2003. Proceedings of the ASP-DAC 2003. Asia and South Pacific, pp. 271 - 276, Jan 2003.

[5] A. Agarwal, V. Zolotov, and D. Blaauw, "Statistical timing analysis using bounds and selective enumeration," IEEE Transactions on Computer-Aided Design of Integrated Circuits and Systems, vol. 22, no. 9, pp. 1243 -1260, Sept 2003.

[6] C. Visweswariah, K. Ravindran, and K. Kalafala, "First-order parameterized block-based statistical timing analysis," TAU'04, Feb 2004.

[7] A. Agarwal, D. Blaauw, and V. Zolotov, "Statistical timing analysis for intra-die process variations with spatial correlations," Computer Aided Design, 2003 International Conference on. ICCAD-2003, pp. 900 - 907, Nov 2003.

[8] S. Bhardwaj, S. B. Vrudhula, and D. Blaauw, "tau: Timing analysis under uncertainty," ICCAD'03, pp. 615620, Nov 2003.

[9] A. Devgan and C. Kashyap, "Block-based static timing analysis with uncertainty," ICCAD'03, pp. 607-614, Nov 2003.

[10] H. Chang and S. S. Sapatnekar, "Statistical timing analysis considering spatial correlations using a single pertlike traversal," ICCAD’03, pp. 621-625, Nov 2003.

[11] S. Tsukiyama, M. Tanaka, and M. Fukui, "A statistical static timing analysis considering correlations between delays," Proceedings of the 2001 conference on Asia South Pacific design automation, January 2001.

[12] S. R. Nassif, "Modeling and analysis of manufacturing variations," CICC, pp. 223-228, 2001.

[13] S. Nassif, "Within-chip variability analysis," Electron Devices Meeting, 1998. IEDM '98 Technical Digest., International, pp. 283 - 286, Dec 1998.

[14] X. Li, J. Le, P. Gopalakrishnan, and L. T. Pileggi, "Asymptotic probability extraction for non-normal distributions of circuit," ICCAD'04, pp. 2-9, Nov 2004.

[15] B. V. Gnedenko, Theory of Probability, 6th ed. Gordon and Breach Science Publishers, 1997, translated from Russian by Igor A. Ushakov. 
[16] C. Clark, "The greatest of a finite set of random variables," Operations Research, pp. 145-162, March 1961. 


\title{
A General Framework for Accurate Statistical Timing Analysis Considering Correlations
}

\author{
Vishal Khandelwal, Ankur Srivastava \\ Department of ECE, University of Maryland-College Park
}

\begin{abstract}
The impact of parameter variations on timing due to process and environmental variations has become significant in recent years. With each new technology node this variability is becoming more prominent. In this work, we present a general Statistical Timing Analysis (STA) framework that captures spatial correlations between gate delays. Our technique does not make any assumption about the distributions of the parameter variations, gate delay and arrival times. We propose a Taylor-series expansion based polynomial representation of gate delays and arrival times which is able to effectively capture the non-linear dependencies that arise due to increasing parameter variations. In order to reduce the computational complexity introduced due to polynomial modeling during STA, we propose an efficient linear-modeling driven polynomial STA scheme. On an average the degree-2 polynomial scheme had a $7.3 x$ speedup as compared to Monte Carlo with 0.049 units of rms error w.r.t Monte Carlo. Our technique is generic and can be applied to arbitrary variations in the underlying parameters.
\end{abstract}

Keywords: Statistical timing, variability, correlation

\section{REFERENCES}

[1] A. Agarwal, D. Blaauw and V. Zolotov. "Statistical Timing Analysis for Intra-Die Process Variations with Spatial Correlations". In Procs of ICCAD, 2003.

[2] A. Agarwal et al. "Computation and Refinement of Statistical Bounds on Circuit Delay". In Procs of DAC, 2003.

[3] A. Agarwal, V. Zolotov and D. Blaauw. "Statistical Timing Analysis Using Bounds and Selective Enumeration". In IEEE Transactions on Computer-Aided Design of Integrated Circuits and Systems, Vol.22, Sept. 2003.

[4] A. Caldwell et al. "Can Recursive Bisection Alone Produce Routable Placements?". In Proc. of DAC, 2000.

[5] C. E. Clark. "The Greates of a Finite Set of Random Variables". In Operations Research, pages 145-162, 1961.

[6] C. Visweswariah et al. "First-Order Incremental Block-Based Statistical Timing Analysis". In Procs of DAC, 2004.

[7] E.M. Sentovich, K.J. Singh, L. Lavagno, C. Moon, R. Murgai, A. Saldanha, H. Savoj, P.R. Stephan, R.K. Brayton, A.L. Sangiovanni-Vincentelli. SIS: A System for Sequential Circuit Synthesis. Memorandum No. UCB/ERL M92/41, Department of EECS. UC Berkeley, May 1992.

[8] H. Chang and S. Sapatnekar. "Statistical Timing Analysis Considering Spatial Correlations Using a Single PertLike Traversal". In Procs of ICCAD, 2003.

[9] J. Le, X. Li and L. Pileggi. "STAC: Statistical Timing Analysis with Correlation". In Procs of DAC, 2004.

[10] M. Cain. "The Moment Generating Function of the Minimum of Bivariate Normal Random Variables". In The American Statistician, pages 124-125, May 1994.

[11] M. Orshansky et al. "Fast Statistical Timing Analysis Handling Arbitrary Delay Correlations". In Procs of $D A C, 2004$. 

Architectures

\author{
Feihui Li and Mahmut Kandemir \\ Computer Science and Engineering Department, The Pennsylvania State University \\ University Park, PA, USA
}

\begin{abstract}
While the past research discussed several advantages of multiprocessor-system-on-a-chip (MPSOC) architectures from both area utilization and design verification perspectives over complex single core based systems, compilation issues for these architectures have relatively received less attention. Programming MPSOCs can be challenging as several potentially conflicting issues such as data locality, parallelism and load balance across processors should be considered simultaneously. Most of the compilation techniques discussed in the literature for parallel architectures (not necessarily for MPSOCs) are loop based, i.e., they consider each loop nest in isolation. However, one key problem associated with such loop based techniques is that they fail to capture the interactions between the different loop nests in the application. This paper takes a more global approach to the problem and proposes a compilerdriven data locality optimization strategy in the context of embedded MPSOCs. An important characteristic of the proposed approach is that, in deciding the workloads of the processors (i.e., in parallelizing the application) it considers all the loop nests in the application simultaneously. Our experimental evaluation with eight embedded applications shows that the global scheme brings significant power/performance benefits over the conventional loop based scheme.
\end{abstract}

Keywords: Data Locality, MPSoC

\title{
REFERENCES
}

[1] A. Agarwal, D. Kranz, and V. Natarajan. Automatic partitioning of parallel loops and data arrays for distributed shared memory multiprocessors. In Proc. International Conference on Parallel Processing, 1993.

[2] S. P. Amarasinghe, J. M. Anderson, M. S. Lam, and C. W. Tseng. The SUIF compiler for scalable parallel machines. In Proc. SIAM Conference on Parallel Processing for Scientific Computing, February, 1995.

[3] U. Banerjee. Dependence Analysis for Supercomputing. Kluwer Academic Publishers, 1988.

[4] F. Catthoor et al. Custom memory management methodology - exploration of memory organization for embedded multimedia system design. Kluwer Academic Publishers, 1998.

[5] M. Cierniak and W. Li. Unifying data and control transformations for distributed shared memory machines. In Proc. Conference on Programming Language Design and Impl., 1995.

[6] I. Kadayif, M. Kandemir, and M. Karakoy. An energy saving strategy based on adaptive loop parallelization. In Proc. $D A C, 2002$.

[7] I. Kadayif, I. Kolcu, M. Kandemir, N. Vijaykrishnan, and M. J. Irwin. Exploiting processor workload heterogeneity for reducing energy consumption in chip multiprocessor. In Proc. DATE, 2004.

[8] I. Kodukula, N. Ahmed, and K. Pingali. Data-centric multilevel blocking. In Proc. ACM Conf. on Programming Language Design and Impl., 1997.

[9] V. Krishnan and J. Torrellas. A chip multiprocessor architecture with speculative multi-threading. IEEE Transactions on Computers, Special Issue on Multi-threaded Architecture, 1999.

[10] J. Li, J. Martinez, and M. Huang. The thrifty barrier: Energy-efficient synchronization in shared-memory multiprocessors. In Proc. High Performance Computer Arch.,, 2004.

[11] S. A. McKee and W. A. Wulf. Access ordering and memory-conscious cache utilization. In Proc. Symposium on High-Performance Computer Arch., 1995.

[12] T. C. Mowry, M. S. Lam, and A. Gupta. Design and evaluation of compiler algorithm for prefetching. In Proc. International Conference on Architectural Support for Programming Languages and Operating Systems, 1992.

[13] MP98: a mobile processor. http://www.labs.nec.co.jp/MP98/top-e.htm.

[14] K. Olukotun, B. A. Nayfeh, L. Hammond, K. Wilson, and K. Chang. The case for a single chip multiprocessor.

In Proc. Conference on Architectural Support for Programming Languages and Operating Systems, 1996.

[15] The Omega Project. http://www.cs.umd.edu/projects/omega/ 
[16] R. Sasanka, S. V. Adve, Y.-K. Chen, and E. Debes. The energy efficiency of CMP vs. SMT for multimedia workloads. In Proc. the 18th Annual International Conference on Supercomputing, 2004.

[17] W.-T. Shiue and C. Chakrabarti. Memory exploration for low-power embedded systems. In Proc. Design Automation Conference, 1999.

[18] O. Temam, E. D. Granston, and W. Jalby. To copy or not copy: A compile-technique for assessing when data copying should be used to eliminate cache conflicts. In Proc. Supercomputing'93, 1993.

[19] M. Wolf and M. Lam. A data locality optimizing algorithm. In Proc. Conference on Programming Language Design and Impl., 1991. 


\title{
Automatic Scenario Detection for Improved WCET Estimation
}

Stefan Valentin Gheorghita, Sander Stuijk, Twan Basten and Henk Corporaal Eindhoven University of Technology, EE Department, Electronic System Group

\begin{abstract}
Modern embedded applications usually have real-time constraints and they are implemented using heterogeneous multiprocessor systems-on-chip. Dimensioning a system requires accurate estimations of the worst-case execution time (WCET). Overestimation leads to overdimensioning. This paper introduces a method for automatic discovery of scenarios that incorporate correlations between different parts of applications. It is based on the application parameters with a large impact on the execution time. We show on a benchmark that, using scenarios, the estimated WCET may be reduced with $16 \%$.
\end{abstract}

Keywords: WCET, Real-Time, Scenarios

\section{REFERENCES}

[1] ARM7TDMI datasheet. http://www.arm.com/products/CPUs/ARM7TDMI.html.

[2] S. Amarasinghe, J. Anderson, M. Lam, and C.-W. Tseng. An overview of the SUIF compiler for scalable parallel machines. In Proc. of the 7th Conference on Parallel Processing for Scienti_c Computing, San Francisco, CA, 1995.

[3] G. Bernat and A. Burns. An approach to symbolic worst-case execution time analysis. In Proc. of 25th Workshop on Real-Time Programming, May 2000.

[4] G. Bernat, A. Colin, and S. M. Petters. WCET analysis of probabilistic hard real-time systems. In Proc. of 23rd IEEE RTSS, pages 269-278, Dec. 2002.

[5] J. Blieberger. Discrete loops and worst case performance. Computer Languages, 20(3):193-212, Aug 1994.

[6] A. Colin and G. Bernat. Scope-tree: A program representation for symbolic worst-case execution time analysis. In Proc. of 14th IEEE ECRTS, pages 50-63, Vienna, Austria, June 2002. IEEE.

[7] S. V. Gheorghita, et al. Sharper WCET upper bounds using automatically detected scenarios. Technical Report ESR-2005-04, TU/e, EE Dept., Electronic Systems Group, Eindhoven, Netherlands, Mar. 2005.

[8] K. Lagerstr" om. Design and implementation of an MP3 decoder, May 2001. M.Sc. thesis, Chalmers University of Technology, Sweden.

[9] Y.-T. S. Li and S. Malik. Performance Analysis of Real-Time Embedded Software. Kluwer Academic Publishers, 1998.

[10] B. Lisper. Fully automatic, parametric worst-case execution time analysis. In Proc. of 3rd Int. Workshop on WCET Analysis, pages 99-102, July 2003.

[11] A. K. Mok, et al. Evaluating tight execution time bounds of programs by annotations. In Proc. of the 6th IEEE RTOSS, pages 74-80, May 1989.

[12] M. Palkovic, et al. Augmenting the exploration space for global loop transformations by systematic preprocessing of data dependent constructs. In Proc. of PA3CT Symposium, pages 33-36, Edegem, Belgium, Sep. 2004.

[13] C. Y. Park. Predicting Deterministic Execution Times of Real-Time Programs. PhD thesis, University of Washington, Seattle, Aug. 1992.

[14] P. Puschner and C. Koza. Calculating the maximum execution time of real-time programs. Journal of RealTime Systems, 1(2):159-176, Sep. 1989.

[15] K. Rijkse. Video coding for narrow telecommunication channels at $<64 \mathrm{kbits} / \mathrm{s}$. Technical report, Telenor R\&D, 1995.

[16] V. Rustagi and D. B. Whalley. Calculating minimum and maximum loop iterations. Technical report, CS Dept., Florida State Univ., May 1994.

[17] A. C. Shaw. Reasoning about time in higher-level language software. IEEE Transactions on Software Engineering, 15(7):875-889, July 1989.

[18] E. Vivancos, et al. Parametric timing analysis. In Proc. of the ACM LCTES'2001, pages 88-93, Utah, June 2001.

[19] P. Yang, et al. Multi-Processor Systems on Chip, chapter Cost-efficient mapping of dynamic concurrent tasks in embedded real-time multimedia systems. Morgan Kaufmann, 2003. 


\author{
Jungeun Kim*, Taewhan Kim** \\ *System R\&D Laboratories, Samsung Electronics Co., Ltd., Seoul, Korea \\ **School of Electrical Engineering, Seoul National University, Seoul, Korea
}

\begin{abstract}
In many of embedded systems, particularly for those with high data computations, the delay of memory access is one of the major bottlenecks in the system's performance. It has been known that there are high variations in memory access delays depending on the ways of designing memory configurations and assigning arrays to memories. Furthermore, embedded DRAM technology that provides efficient access modes is actively developed, possibly becoming a mainstream in future embedded system design. In that context, in this paper we propose an effective solution to the problem of (embedded DRAM) memory allocation and mapping in memory access code generation with the objective of minimizing the total memory access time. Specifically, the proposed approach, called MACCESS-opt, solves the three problems simultaneously: (i) determination of memories, (ii) mapping of arrays to memories, and (iii) scheduling of memory access operations, so that the use of DRAM access modes is maximized while satisfying the storage size constraint of embedded system. Experimental data on a set of benchmark designs are provided to show the effectiveness of the proposed integrated approach. In short, MACCESS-opt reduces the total memory access latency by over $18 \%$, from which we found that our memory mapping and scheduling techniques in MACCESS-opt contribute about $12 \%$ and $6 \%$ reductions of total memory access latency, respectively.
\end{abstract}

Keywords: memory access, scheduling, binding

\title{
REFERENCES
}

[1] B. Prince, High Performance Memories, New Architecture DRAMs and SRAMs Evolution and Function, Wiley, West Sussex, 1996.

[2] S. Przybylski, "Sorting out the new DRAMs", In Hot Chips Tutorial, Stanford, CA, 1997.

[3] H. Schmit and D. E. Thomas, "Array Mapping Behavioral Synthesis”, ISSS, 1995.

[4] P. R. Panda, "Memory Bank Customization and Assignment in Behavioral Synthesis", ICCAD, 1999.

[5] P. R. Panda et al., "Incorporating DRAM Access Modes into High-level Synthesis", IEEE TCAD, Vol. $17,1998$.

[6] W. T. Shiue and C. Chakrabarti, "Memory Exploration for Low Power Embedded Systems", DAC, 2001.

[7] F. Balasa, et al., "Dataflow-driven Memory Allocation for Multi-dimensional Signal Processing Systems", ICCAD, 1994.

[8] J. Seo, et al., “An Integrated Algorithm for Memory Allocation and Assignment in High-level Synthesis”, DAC, 2002.

[9] S. Bakshi, and D. Gajski, “A Memory Selection Algorithm for High-Performance Pipelines” EDAC, 1995.

[10] P. R. Panda, et al., "Data and Memory Optimization Techniques for Embedded Systems", ACM TODAES, Vol. $6,2002$.

[11] I. Kadayif, et al., "Locality-Conscious Process Scheduling in Embedded Systems", Proc. Symposium on HW/SW Codesign, 2002.

[12] Y. Choi, T. Kim, "Memory Layout Techniques for Variables Utilizing Efficient DRAM Access Modes", $D A C$, 2003.

[13] W-T. Shiue, et al., "Low Power Multi-Module, Multi-Port Memory Design for Embedded Systems”, Workshop on Signal Processing, 2000.

[14] P. Grun, et al., "Memory Aware Compilation Through Accurate Timing Extraction", DAC, 2000.

[15] W. H. Press, et al., "Numerical Recipes in C", Cambridge university press, 1992. 

Embedded Systems

\author{
Ravindra Jejurikar*, Rajesh Gupta**
}

*Center for Embedded Computer Systems, University of California, Irvine, Irvine, CA

**Department of Computer Science, University of California, San Diego, La Jolla, CA

\begin{abstract}
Leakage energy consumption is an increasing concern in current and future CMOS technology generations. Procrastination scheduling, where task execution can be delayed to maximize the duration of idle intervals, has been proposed to minimize leakage energy drain. We address dynamic slack reclamation techniques under procrastination scheduling to minimize the static and dynamic energy consumption. In addition to dynamic task slowdown, we propose dynamic procrastination which seeks to extend idle intervals through slack reclamation. While using the entire slack for either slowdown or procrastination need not be the most energy efficient approach,we distribute the slack between slowdown and procrastination to exploit maximum energy savings. Our simulation experiments show that dynamic slowdown results on an average $10 \%$ energy gains over static slowdown. Dynamic procrastination extends the average sleep interval by $25 \%$ which reduces the idle energy consumption by $15 \%$, while meeting all timing requirements.
\end{abstract}

Keywords: dynamic slack reclamation, task procrastication, leakage power, critical speed, low power scheduling, real-time systems

\title{
REFERENCES
}

[1] H. Aydin, R. Melhem, D. Mossé, and P. M. Alvarez. Determining optimal processor speeds for periodic realtime tasks with different power characteristics. In Proceedings of EuroMicro Conference on Real-Time Systems, Jun. 2001.

[2] H. Aydin, R. Melhem, D. Mossé, and P. M. Alvarez. Dynamic and aggressive scheduling techniques for poweraware real-time systems. In Proceedings of IEEE Real-Time Systems Symposium, Dec. 2001.

[3] F. Gruian. Hard real-time scheduling for low-energy using stochastic data and dvs processors. In Proceedings of International Symposium on Low Power Electronics and Design, pages 46-51, Aug. 2001.

[4] S. Irani, S. Shukla, and R. Gupta. Algorithms for power savings. In Proceedings of Symposium on Discrete Algorithms, Jan. 2003.

[5] R. Jejurikar and R. Gupta. Leakage aware dynamic slack reclamation in real-time embedded systems. In CECS Technical Report \#04-31, UC Irvine, Nov. 2004.

[6] R. Jejurikar, C. Pereira, and R. Gupta. Leakage aware dynamic voltage scaling for real-time embedded systems. In Design Automation Conference, pages 275-280, Jun. 2004.

[7] W. Kim, J. Kim, and S. L. Min. A dynamic voltage scaling algorithm for dynamic-priority hard real-time systems using slack time analysis. In DATE, Mar. 2002.

[8] Y. Lee, K. P. Reddy, and C. M. Krishna. Scheduling techniques for reducing leakage power in hard real-time systems. In EcuroMicro Conf. on Real Time Systems, 2003.

[9] J. W. S. Liu. Real-Time Systems. Prentice-Hall, 2000.

[10] C. Locke,D. Vogel, and T. Mesler. Building a predictable avionics platform in ada: a case study. In Proceedings IEEE Real-Time Systems Symposium, 1991.

[11] Y. Shin, K. Choi, and T. Sakurai. Power optimization of real-time embedded systems on variable speed processors. In Proc. of ICCAD, pages 365-368, Nov. 2000.

[12] F. Yao, A. J. Demers, and S. Shenker. A scheduling model for reduced CPU energy. In Proceedings of IEEE Symposium on Foundations of Computer Science, pages 374-382, 1995.

[13] F. Zhang and S. T. Chanson. Processor voltage scheduling for real-time tasks with non-preemptible sections. In Proceedings of Real Time Systems Symposium, Dec. 2002. 


\title{
Response Compaction with any Number of Unknowns using a new LFSR Architecture
}

\author{
Erik H. Volkerink ${ }^{1,2}$ and Subhasish Mitra ${ }^{2,3}$ \\ ${ }^{1}$ Agilent Laboratories, Palo Alto, CA \\ ${ }^{2}$ Intel Corporation, Folsom, CA \\ ${ }^{3}$ Center for Reliable Computing, Stanford University, Stanford, CA
}

\begin{abstract}
This paper presents a new test response compaction technique with any number of unknown logic values (X's) in the test response bits. The technique leverages an X-tolerant response compactor (X-compact), and forces X's that are not tolerated by X-Compact to known values. The data required to designate the $\mathrm{X}$ 's not tolerated by the X-compactor, also called mask data, is stored in a compressed format on the tester and decompressed onchip. We applied this technique to four industrial designs and obtained 26 -fold to 60 -fold reduction in test response data volume with no impact on test quality.
\end{abstract}

Keywords: VLSI Test, Compression, X-compact, LFSR, BIST

\section{REFERENCES}

[1] Bardell, P.H., W. H. McAnney, J. Savir, "Built-in Test For VLSI: Pseudo-random Techniques," Wiley InterScience, 1987.

[2] Barnhart K., B. Keller, B. Koenemann, R. Walther, “OPMISR: The Foundation for Compressed ATPG Vectors”, Proc. Int. Test. Conf., 2001.

[3] Benowitz, N., D. Calhoun, G. Alderson, J. Bauer, C. Joeckel, "An Advanced Fault Isolation System for Digital Logic” IEEE Trans. Computers, Vol.24, No.5, pp. 489-497, 1975.

[4] Chickermane, V., B. Foutz, B. Keller, "Channel Masking Synthesis for Efficient On-Chip Test Compression," Proc. Int. Test. Conf., 2004.

[5] Koenemann, B., "LFSR-Coded Test Patterns for scan Designs", Proc. of European Test Conference, pp. 237242, 1991.

[6] McCluskey, E.J., "Logic Design Principles with Emphasis on Testable Semi-Custom Circuits," Prentice Hall, Englewood Cliffs, NJ, USA, 1986.

[7] McCluskey, E.J., C.W. Tseng, "Stuck-Fault Tests vs. Actual Defects," Proc. Intl. Test Conf., pp. 336-343, 2000.

[8] Mitra, S., K. S. Kim, "X-compact: An Efficient Response Compaction for Test Cost Reduction," Proc. of the International Test Conference, 2002.

[9] Mitra, S., S. Lumetta, M. Mitzenmacher, "X-tolerant Signature Analysis," Proc. of the International Test Conference, 2004.

[10] Naruse, M., I. Pomeranz, S.M. Reddy, S. Kundu, "On-chip Compression of Output Responsoes with Unknown Values using LFSR Reseeding," Proc. Intl. Test Conf., 2003.

[11] Patel, J.H., S.S. Lumetta, S.M. Reddy, "Application of Saluja-Karpovsky Compactors to Test Responses with Many Unknowns," Proc. IEEE VLSI Test Symp., 2003.

[12] Pomeranz, I. S. Kundu, S.M. Reddy, "On Output Response Compression in the Presence of Unknown Output Values," Proc. Design Automation Conf. Pp. 255-258, 2002.

[13] Rajski, J, M. Kassab, N. Mukherjee, R. Thompson, H. Tasai, A. Hertwig, N. Tamarapalli, J. Tyszer, "Embedded Deterministic Test", Proc. International Test Conference, 2002.

[14] Rajski, J., J. Tyszer, C. Wang, S.M. Reddy, "Convolution Compaction of Test Responses," Proc. Intl. Test Conf., 2003

[15] Saxena, N.R., and E.J. McCluskey, "Parallel Signature Analysis Design with Bounds on Aliasing" IEEE Trans. Computers, Vol. 46, No.5, pp. 425-438, 1997.

[16] Sinanoglue, O., A. Orailoglu, "Compacting Test Responses for Deeply Embbedded SOC Cores," IEEE Design \& Test of Computers, Vol. 20, Issue 4, 2003.

[17] Tang, Y., H-J Wunderlich, H. Vranken, F. Hapke, M. Wittke, P. Engelke, I. Polian, B. Becker, "X-Masking During Logic BIST and Its Impact on Defect Coverage," Proc. International Test Conference, 2004.

[18] Tseng, C.W., and E.J. McCluskey, "Multiple-Output Propagation Transition Fault Test," Proc. Intl. Test Conf., pp. 358-366, 2001.

[19] Volkerink, E.H., S. Mitra, “Efficient LFSR reseeding," Proc. VLSI Test Symposium, 2003. 
[20] Volkerink, E.H., S. Mitra, “Test Response Compression for Any Number of Unknowns," IEEE Intl. Workshop Infrastructure IP, 2003.

[21] Wang, C., S.M. Reddy, I. Pomeranz, J. Rajski, J. Tyszer, “On Compacting Test Response Data Containing Unknown Values," Proc. Intl. Conf. Computer-Aided Design, 2003.

[22] Wohl, P., J. Waicukauski, S. Patel, M. Amin, "X-Tolerant Compression and Application of Scan ATPG patterns in a BIST architecture”, Proc. Intl. Test Conf., 2003.

[23] Wohl, P., L. Huisman, "Analysis and Design of Optimal Combinational Compactors," Proc. IEEE VLSI Test Symp., 2003. 


\title{
Multi-Frequency Wrapper Design and Optimization for Embedded Cores Under Average
} Power Constraints

\author{
Qiang Xu*, Nicola Nicolici*, Krishnendu Chakrabarty** \\ *ECE Department, McMaster University, ON L8S 4K1, Canada \\ **ECE Department, Duke University, Durham, NC 27708, USA
}

\begin{abstract}
This paper presents a new method for designing test wrappers for embedded cores with multiple clock domains. By exploiting the use of multiple shift frequencies, the proposed method improves upon a recent wrapper design method that requires a common shift frequency for the scan elements in the different clock domains. We present an integer linear programming (ILP) model that can be used to minimize the testing time for small problem instances. We also present an efficient heuristicmethod that is applicable to large problem instances, and which yields the same (optimal) testing time as ILP for small problem instances. Compared to recent work on wrapper design using a single shift frequency, we obtain lower testing times and the reduction in testing time is especially significant under power constraints.
\end{abstract}

Keywords: Wrapper Design, Multiple Clock Domains, Scan Control Unit

\section{REFERENCES}

[1] S. K. Goel and E. J. Marinissen. Control-Aware Test Architecture Design for Modular SOC Testing. In Proceedings IEEE European Test Workshop (ETW), pages 57-62, Maastricht, The Netherlands, May 2003.

[2] V. Iyengar, K. Chakrabarty, and E. J. Marinissen. Co-Optimization of Test Wrapper and Test Access Architecture for Embedded Cores. Journal of Electronic Testing: Theory and Applications, 18(2):213-230, Apr. 2002.

[3] V. Jain and J. Waicukauski. Scan Test Data Volume Reduction in Multi-clocked Designs with Safe Capture Technique. In Proceedings IEEE International Test Conference (ITC), pages 148 -153, Oct. 2002.

[4] A. Khoche. Test Resource Partitioning for Scan Architectures Using Bandwidth Matching. In Digest of Int. Workshop on Test Resource Partitioning, pages 1.4.1-1.4.8, 2002.

[5] S. Koranne. A Novel Reconfigurable Wrapper for Testing of Embedded Core-Based SOCs and its Associated Scheduling Algorithm. Journal of Electronic Testing: Theory and Applications, 18(4/5):415-434, Aug. 2002.

[6] E. Larsson and Z. Peng. A Reconfigurable Power-Conscious Core Wrapper and its Application to SOC Test Scheduling. In Proceedings IEEE International Test Conference (ITC), pages 1135-1144, Charlotte, NC, Sept. 2003.

[7] X. Lin, R. Press, J. Rajski, P. Reuter, T. Rinderknecht, B. Swanson, and N. Tamarapalli. High-Frequency, AtSpeed Scan Testing. IEEE Design \& Test of Computers, 20(5):17-25, Oct 2003.

[8] X. Lin and R. Thompson. Test generation for designs with multiple clocks. In Proceedings ACM/IEEE Design Automation Conference (DAC), pages 662 -667, 2003.

[9] E. J. Marinissen et al. A Structured And Scalable Mechanism for Test Access to Embedded Reusable Cores. In Proceedings IEEE International Test Conference (ITC), pages 284-293, Washington, DC, Oct. 1998.

[10] E. J. Marinissen et al. On IEEE P1500's Standard for Embedded Core Test. Journal of Electronic Testing: Theory and Applications, 18(4/5):365-383, Aug. 2002.

[11] E. J. Marinissen, S. K. Goel, and M. Lousberg. Wrapper Design for Embedded Core Test. In Proceedings IEEE International Test Conference (ITC), pages 911-920, Atlantic City, NJ, Oct. 2000.

[12] N. Nicolici and B. M. Al-Hashimi. Power-Constrained Testing of VLSI Circuits. Kluwer Academic Publishers, 2003.

[13] S. Pateras. Achieving At-Speed Stuctural Test. IEEE Design \& Test of Computers, 20(5):26-33, Oct 2003.

[14] J. Schmid and J. Knablein. Advanced Synchronous Scan Test Methodology for Multi Clock Domain ASICs. In Proceedings IEEE VLSI Test Symposium (VTS), pages 106 - 113, 1999.

[15] H. Schwab. Lp solve. In http://elib.zib.de/pub/Packages/mathprog/linprog/lp-solve, 1997.

[16] A. Sehgal and K. Chakrabarty. Efficient Modular Testing of SOCs Using Dual-Speed TAM Architectures. In Proceedings Design, Automation, and Test in Europe (DATE), pages 422-427, Paris, France, Feb. 2004.

[17] Technical White Paper. Designs with Multiple Clock Domains: Avoiding Clock Skew and Reducing Pattern Count Using DFT Advisor and Fast Scan. http://www.mentor.com/dft. 
[18] N. Tendolkar, R. Raina, R. Woltenberg, X. Lin, B. Swanson, and G. Aldrich. Novel Techniques for Achieving High At-Speed Transition Fault Test Coverage for Motorola Microprocessors Based on PowerPC Instruction Set Architecture. In Proceedings IEEE VLSI Test Symposium (VTS), pages 3-8, 2002.

[19] B. Vermeulen, S. Oostdijk, and F. Bouwman. Test and Debug Strategy of the PNX8525 NexperiaTM Digital Video Platform System Chip. In Proceedings IEEE International Test Conference (ITC), pages 121-130, Oct. 2001.

[20] D. Wu, M. Lin, S. Mitra, K. S. Kim, A. Sabbavarapu, T. Jaber, P. Johnson, D. March, and G. Parrish. H-DFT:

A Hybrid DFT Architecture for Low-Cost High Quality Structural Testing. In Proceedings IEEE International Test Conference (ITC), pages 1229 - 1238, 2003.

[21] Q. Xu and N. Nicolici. Wrapper Design for Testing IP Cores with Multiple Clock Domains. In Proceedings Design, Automation, and Test in Europe (DATE), pages 416-421, Paris, France, Feb. 2004. 


\title{
N-Detection Under Transparent-Scan \\ Irith Pomeranz \\ School of ECE, Purdue University, W. Lafayette, IN 47907
}

\begin{abstract}
We study the quality of test sequences under a test application scheme called transparent-scan as $n$-detection test sequences. We obtain transparent-scan sequences from combinational test sets. We show that for the same number of clock cycles required to apply a compact single-detection combinational test set, a transparent-scan sequence detects faults more times than the combinational test set. We note that a transparent-scan sequence based on a combinational test set contains unspecified values. We consider several procedures for specifying the unspecified values of the transparent-scan sequence, and study their effects. We also study the extension of a transparent-scan test sequence into an $n$-detection test sequence that detects every target fault at least $n$ times.
\end{abstract}

Keywords: $n$-detection test sets, scan design, test generation

\section{References}

[1] S. C. Ma, P. Franco and E. J. McCluskey, "An Experimental Chip to Evaluate Test Techniques Experiment Results", in Proc. Intl. Test Conf., 1995, pp. 663-672.

[2] L.-C. Wang, M. R. Mercer and T. W. Williams, "On the Decline of Testing Efficiency as the Fault Coverage Approaches 100\%", in Proc. VLSI Test Symp., April 1995, pp. 74-83.

[3] S. M. Reddy, I. Pomeranz and S. Kajihara, "Compact Test Sets for High Defect Coverage", IEEE Trans. on Computer-Aided Design, Aug. 1997, pp. 923-930.

[4] J. T.-Y. Chang, C.-W. Tseng, C.-M. J. Li, M. Purtell and E. J. McCluskey, "Analysis of Pattern-Dependent and Timing-Dependent Failures in an Experimental Test Chip", in Proc. Intl. Test Conf., 1998, pp. 184-193.

[5] I. Pomeranz and S. M. Reddy, "Test Sequences to Achieve High Defect Coverage for Synchronous Sequential Circuits", IEEE Trans. on Computer-Aided Design, Oct. 1998, pp. 1017-1029.

[6] M. R. Grimaila, S. Lee et. al., "REDO - Random Excitation and Deterministic Observation - First Commercial Experiment", in Proc. VLSI Test Symp., 1999, pp. 268-274.

[7] C.-W. Tseng and E. J. McCluskey, "Multiple-Output Propagation Transition Fault Test", in Proc. Intl. Test Conf., 2001, pp. 358-366.

[8] B. Benware, C. Schuermyer, N. Tamarapalli, K.-H. Tsai, S. Ranganathan, R. Madge, J. Rajski and P. Krishnamurthy, "Impact of multiple-detect test patterns on product quality", in Proc. Intl. Test Conf., Sept. 2003, pp. 1031-1040.

[9] S. Venkataraman, S. Sivaraj, E. Amyeen, S. Lee, A Ojha and R. Guo, "An Experimental Stud of $n$-Detect Scan ATPG Patterns on a Processor", in Proc. 22nd VLSI Test Symp., April 2004, pp. 23-28.

[10] I. Pomeranz and S. M. Reddy, "A New Approach to Test Generation and Test Compaction for Scan Circuits", in Proc. Design Automation and Test in Europe Conf., March 2003, pp. 1000-1005.

[11] J. Grodstein, D. Bhavsar, V. Bettada and R. Davies, "Automatic Generation of Critical-Path Tests for a PartialScan Microprocessor", in Proc. Intl. Conf. on Computer Design, Oct. 2003, pp. 180-186. 


\title{
Secure Scan: A Design-for-Test Architecture for Crypto Chips
}

\author{
Bo Yang*, Kaijie Wu**, Ramesh Karri* \\ *ECE Department, Polytechnic University, Brooklyn, NY, 11201 \\ **ECE Department, University of Illinois, Chicago, Chicago, IL, 60612
}

\begin{abstract}
Scan-based Design-for-Test (DFT) is a powerful testing scheme, but it can be used to retrieve the secrets stored in a crypto chip thus compromising its security. On one hand, sacrificing security for testability by using traditional scan-based DFT restricts its use in privacy sensitive applications. On the other hand, sacrificing testability for security by abandoning scan-based DFT hurts product quality. The security of a crypto chip comes from the small secret key stored in a few registers and the testability of a crypto chip comes from the data path and control path implementing the crypto algorithm. Based on this key observation, we propose a novel scan DFT architecture called secure scan that maintains the high test quality of traditional scan DFT without compromising the security. We used a hardware implementation of the Advanced Encryption Standard (AES) to show that the traditional Scan DFT scheme can compromise the secret key. We then showed that by using secure scan DFT, neither the secret key nor the testability of the AES implementation is compromised.
\end{abstract}

Keywords: Scan-based DFT, Testability, Security, Crypto Hardware

\section{REFERENCES}

[1] S. Mangard, M. Aigner and S. Dominikus, A Highly Regular and Scalable AES Hardware Architecture, IEEE Transactions on Computer, vol. 52, no.1, pp. 483-491, April 2004.

[2] M.L. Bushnell and V.D. Agrawal, Essentials of Electronic Testing, Kluwer Academic Publishers, 2000.

[3] D, Josephson and S. Poehhnan, Debug methodology for the McKinley processor, International Test Conference, pp.451-460, 2001

[4] B. Yang, K. Wu and R. Karri, Scan Based Side Channel Attack on Dedicated Hardware Implementations of Data Encryption Standard, International Test Conference, pp.339-344, 2004

[5] R. Goering, Scan Design Called Portal for Hackers, EE Times, Oct. 2004. http://www.eetimes.com/news/latest/ showArticle.jhtml?articleID $=51200146$

[6] Maestra Comprehensive Guide to Satellite TV Testing, 2002. http://www.maestra.tv/downloads/Maestra_Guide. pdf

[7] O. Kömmerling, M. G. Kuhn, Design Principles for Tamper-Resistant Smartcard Processors, USENIX Workshop on Smartcard Technology, pp.9-20, May, 1999.

[8] R. J. Easter, E. W. Chencinski, E. J. D'Avignon, S. R. Greenspan, W. A. Merz and C. D. Norberg, S/390 Parallel Enterprise Server CMOS Cryptographic Coprocessor, IBM Journal of Research and Development, Vol 43, pp.761776,1999

[9] D. Hély, F. Bancel, ML Flottes, B. Rouzeyre, M. Renovell and N. Bérard, Scan Design and Secure Chip, IEEE International On-Line Testing Symposium pp.219-226, 2004.

[10] R. Zimmermann, A. Curiger, H. Bonnenberg, H. Kaeslin, N. Felber and W. Fichtner, A $177 \mathrm{Mb} / \mathrm{sec}$ VLSI implementation of the international data encryption algorithm, IEEE Journal of Solid-State Circuits, vol. 29, no. 3, pp. 303-307, March, 1994.

[11] National Bureau of Standards, Security Requirements for Cryptographic Modules, Federal Information Processing Standards Publication FIPS PUB 140-2, 2002.

[12] Biham and A. Shamir, Differential Fault Analysis of Secret Key Cryptosystems, CRYPTO, pp. 156-171, 1991. 


\title{
A Green Function-Based Parasitic Extraction Method
} for Inhomogeneous Substrate Layers

\author{
Chenggang Xu, Ranjit Gharpurey*, Terri S. Fiez, and Kartikeya Mayaram \\ School of EECS, Oregon State University, Corvallis, OR 97331 \\ *Dept. of EECS, University of Michigan, Ann Arbor, MI 48109
}

\begin{abstract}
This paper presents a new Green function-based approach for substrate parasitic extraction in substrates with inhomogeneous layers. This new formulation allows analysis of noise coupling with sinkers, trenches and wells, - a limitation in prior Green function-based extractors. Numerical examples for sinkers and trenches are provided and compared with the results from three-dimensional semiconductor device simulations. It is shown that the proposed method is accurate and computationally efficient.
\end{abstract}

Keywords: Substrate noise, parasitic extraction, Green function

\section{References}

[1] B. R. Stanisic, N. K. Verghese, R. A. Rutenbar, R. L. Carley, and D. J. Allstot, “Addressing substrate coupling in mixed-mode IC's and power distribution synthesis," IEEE J. Solid-State Circuits, vol. 29, pp. 226-237, March 1994.

[2] P. Miliozzi, L. Carloni, E. Charbon, and A. Sangiovanni-Vincentelli, "SUBWAVE: a methodology for modeling digital substrate noise injection in mixed-signal ICs," Proceedings of the IEEE Custom Integrated Circuits Conference, pp. 385-388, May 1996.

[3] M. Pfost and H. M. Rein, "Modeling and measurement of substrate coupling in Si-Bipolar IC's up to $40 \mathrm{GHz}$," IEEE J. Solid-State Circuits, vol. 33, pp. 582-591, April 1998.

[4] S. Donnay and G. Gielen, Substrate Noise Coupling in Mixed-signal ASICs, Kluwer Academic Publishers, 2003.

[5] N. K. Verghese, T. Schmerbeck, and D.J. Allstot, Simulation Techniques and Solutions for Mixed-Signal Coupling in ICs, Kluwer Academic Publisher, 1995.

[6] X. Aragones, J. 1. Gonzalez, and A. Rubio, Analysis and Solutions for Switching Noise Coupling in Mixed-Signal ICs, Kluwer Academic Publisher, 1999.

[7] E. Charbon, R. Gharpurey, P. Milliozzi, R.G. Meyer, and A. Sangiovanni-Vincentelli, Substrate Noise: Analysis and Optimization for IC Design, Kluwer Academic Publisher, 1995.

[8] T. A. Johnson, R. W. Knepper, V. Marcello, and W. Wang, "Chip substrate resistance modeling technique for integrated circuit design," IEEE Trans. Computer-Aided Design, vol. 3, pp. 126-134, April 1984.

[9] L. M. Silveira and N. Vargas, "Characterizing substrate coupling in deep-submicron designs," IEEE Design \& Test of Computers, vol. 19, pp. 26-29, March-April 2002.

[10] R. Gharpurey, "Modeling and analysis of substrate coupling in IC's," Ph.D. dissertation, Univ. California, Berkeley, 1995.

[11] A. M. Niknejad, R. Gharpurey, and R. G. Meyer, "Numerically stable Green function for modeling and analysis of substrate coupling in integrated circuits," IEEE Trans. Computer-Aided Design, vol. 17, pp. 305-315, April 1998.

[12] J. P. Costa, M. Chou, and L. M. Silveira, "Efficient techniques for accurate modeling and simulation of substrate coupling in mixed-signal IC's," IEEE Trans. Computer-Aided Design, vol. 18, pp. 597-607, May 1999.

[13] H. Li, J. Carballido, H. H. Yu, V. I. Okhmatovski, E. Rosenbaum, and A. C. Cangellaris, "Comprehensive frequency-dependent substrate noise analysis using boundary element methods," IEEE/ACM International Conference on Computer-Aided Design, pp. 2-9, Nov. 2002.

[14] E. Schrik and N. R. van der Meijs, "Combined BEM/FEM substrate resistance modeling," Proc. 39th Design Automation Conference, pp. 771 - 776, June 2002.

[15] E. Schrik, P. M. Dewilde, and N. P. van der Meijs, "Theoretical and practical validation of combined BEM/FEM substrate resistance modeling," IEEE/ACM International Conference on Computer Aided Design, pp. 10-15, Nov. 2002.

[16] W. H. Press, B. P. Flannery, S. A. Teukolsky, and W. T. Vetterling, Numerical Recipes in C, Cambridge University Press, 1988.

[17] ATLAS User's Manual, SILVACO International. 


\title{
Analysis of Full-Wave Conductor System Impedance over Substrate Using Novel Integration Techniques
}

\author{
Xin Hu, Jung Hoon Lee, Jacob White, Luca Daniel
}

M.I.T.

\begin{abstract}
An efficient approach to full-wave impedance extraction is developed that accounts for substrate effects through the use of two-layer media Green's functions in a mixed-potential-integralequation (MPIE) solver. Particularly, the choice of implementation for the layered media Green's functions motivates the development of accelerated techniques for both volume and surface integrations in the solver. Solver accuracy is validated against measurements taken on fabricated devices; solver efficiency is demonstrated by its $9.8 \mathrm{X}$ reduction in cost in comparison to the traditional integration approach.
\end{abstract}

Keywords: Impedance extraction, Substrate modeling, Integral equation solver

\section{REFERENCES}

[1] K.A. Michalski. "Electromagnetic Scattering and Radiation by Surfaces of Arbitrary Shape in Layered Media, Part I: Theory." IEEE Trans. Antennas Propagat., Vol.38, No. 3, March 1990.

[2] J.J. Yang, Y.L. Chow, D.G. Fang. "Discrete Complex Images of a Three-dimensional Dipole Above and Within a Lossy Ground." IEE PROC-H, Vol. 138, No. 4, Aug. 1991.

[3] Y.L. Chow, J.J. Yang, and G.E. Howard. "A Closed-Form Spatial Green's Function for the Thick Microstrip Substrate." IEEE Trans. Microwave Theory Tech., Vol. 39, No. 3, March 1991.

[4] G. Dural and M.I. Aksun. "Closed-Form Green's Functions for General Sources and Stratified Media". IEEE Trans. Microwave Theory Tech., Vol. 43, No. 7, July 1995.

[5] M.I. Aksun. "A Robust Approach for the Derivation of Closed-Form Green's Functions." IEEE Trans. Microwave Theory and Tech., Vol. 44, No. 5, May 1996.

[6] N. Hojjat, S. Safavi-Naeini, R. Faraji-Dana, and Y.L. Chow. "Fast Computation of the Nonsymmetrical Components of the Green's Function for Multilayer Media using Complex Images." IEEE Trans. Antennas Propagat., Vol. 145, no.4, Aug. 1998.

[7] D. Wilton, S.M. Rao, A.W. Glisson, etc. "Potential Integrals for Uniform and Linear Source Distributions on Polygonal and Polyhedral Domains." IEEE Trans. Antennas Propagat., Vol. AP 32, no.3, March, 1984.

[8] J. Tan. "Full Wave Analysis of Transmission Lines in a Multilayer Substrate with Heavy Dielectric Losses." IEEE Trans. Components, Packaging and Manufacturing Technology, Vol. 19, No. 3, Aug. 1996

[9] A. Polycarpou. "The Finite-Element method for Modeling Circuits and Interconnects for Electronic Packaging." IEEE Trans. Microwave Theory Tech., Vol. 45, No. 10, Oct. 1997.

[10] A. Niknejad and R. Meyer. "Analysis, Design, and Optimization of Spiral Inductors and Transformers for Si RF IC's." IEEE J. Solid-State Circuits, Vol. 33, No. 10, Oct. 1998.

[11] C. Chen, T. Lee, N.Murugesan and S. Hagness. "Generalized FDTD-ADI: An Unconditionally Stable FullWave Maxwell's Equations Solver for VLSI Interconnect Modeling." Computer Aid Design, 2000.

[12] J. Zheng, V. Tripathi, and A. Weisshaar. "Characterization and Modeling of Multiple Coupled On-Chip Interconnects on Silicon Substrate." IEEE Trans. Microwave Theory Tech., Vol. 49, No. 10, Oct. 2001.

[13] H. Ymeri, B. Nauwelaers, K. Maex, S. Vandenberghe, and D. Roest. "New Analytic Expressions for Mutual Inductance and Resistance of Coupled Interconnects on Lossy Silicon Substrate." Si Monolithic Integrated Circuits in RF Systems, 2001.

[14] J.Fan, J.L. Drewniak, H. Shi and J.L. Knighten. "DC Power-Bus Modeling and Design With a Mixed-Potential Integral-Equation Formulation and Circuit Extraction." IEEE Trans. Electromagnetic Compatibility, Vol. 43 , No. 4 , Nov. 2001.

[15] H. Lan. "A CAD-Oriented Modeling Approach of Frequency-Dependent Behavior of Substrate Noise Coupling for Mixed-Signal IC Design.” Int. Symp. on Quality Electronic Design, 2003.

[16] A. Weisshaar, H. Lan, and A. Luoh. "Accurate Closed-form Expressions for the Frequency-Dependent Line Parameters of On-Chip Interconnects on Lossy Silicon Substrate." IEEE Trans. on Advanced Packaging, vol. 25, No. 2, May 2002.

[17] A.E. Ruehli. "Equivalent Circuit Models for Three Dimensional Multiconductor Systems." IEEE Trans. Microwave Theory Tech., Vol. 22, March 1974. 
[18] M. Kamon, N. Marques, and J.White. "FastPep: A Fast parasitic Extraction Program for Complex ThreeDimensional Geometries." Proc. of the IEEE Conference on Computer-Aided Design, San Jose, Nov. 1997.

[19] N. Marques, M. Kamon, J.K. White, and L.M. Silverira. "A mixed nodal-mesh formulation for efficient extraction and passive reduced-order modeling of 3D interconnects." Proc. of the IEEE/ACM DAC, San Francisco, CA, June 1998.

[20] J. Peters. Design of High Quality Factor: Spiral Inductors in RF MCM-D. Master's thesis, M.I.T. Sept. 2004

[21] R.F. Harrington. Field Computation by Moment Methods. MacMillan, 1968. 


\title{
Spatially Distributed 3D Circuit Models
}

\author{
Michael Beattie $^{a}$, Hui Zheng ${ }^{b}$, Anirudh Devgan ${ }^{b}$ and Byron Krauter ${ }^{a}$ \\ ${ }^{\mathrm{a}}$ Electronic Design Automation $\quad{ }^{\mathrm{b}}$ Austin Research Lab \\ IBM Corporation, Austin, Texas 78758
}

\begin{abstract}
Spatially distributed 3D circuit models are extracted with a segment-to-segment BEM (Boundary Element Method) algorithm for both capacitance and inverse inductance couplings rather than using the traditional net-to-net approach. Critical issues regarding the extraction efficiency and accuracy of segment-to-segment BEM capacitance models are explored. An adaptive discretization scheme is developed for segment-to-segment capacitance extraction and also applied to segment-to-segment high-frequency inverse inductance extraction. We demonstrate the limitations of the duality between capacitance and inverse inductance. Examples demonstrating the accuracy of these models are presented for real packaging cases.

Keywords: Distributed Circuit Models, Boundary Element Method (BEM), Capacitance, Inverse Inductance

\section{References}

[1] F. Yu, W. Shi, A Divide-and-Conquer Algorithm for 3D Capacitance Extraction, 5th ISQED (Mar. 2004)

[2] B. Krauter, L. Pileggi, Generating Sparse Partial Inductance Matrices with Guaranteed Stability, ICCAD 1996

(Nov. 1996)

[3] A. Devgan, H. Ji, W. Dai, How to Efficiently Capture On-Chip Inductance Effect: Introducing a New Circuit Element $K$, ICCAD 2000 (Nov. 2000)

[4] H. Ji, A. Devgan, W. Dai, KSim: A Stable and Efficient RKC Simulator for Capturing On-Chip Inductance Effect, ASPDAC 2001 (Jan. 2001)

[5] M. Beattie, L. Pileggi, Efficient Inductance Extraction via Windowing, DATE 2001 (Mar. 2001)

[6] H. Zheng, L. Pileggi, M. Beattie, B. Krauter, Window-Based Susceptance Models for Large-Scale RLC Circuit Analyses, DATE 2002 (Mar. 2002)

[7] M. Beattie, L. Pileggi, Bounds for BEM Capacitance Extraction, 34th DAC (Jun. 1997)

[8] M. Beattie, L. Pileggi, Error Bounds for Capacitance Extraction via Window Techniques, IEEE TCAD, vol. 18, No. 3 (Mar. 1999)

[9] K. Nabors, J. White, FASTCAP: A Multipole Accelerated 3-D Capacitance Extraction Program, IEEE TCAD, vol. 10, No. 11 (Nov. 1991)

[10] W. Shi, J. Liu, N. Kakani, T. Yu, A Fast Hierarchical Algorithm for 3-D Capacitance Extraction, 35th DAC (Jun. 1998)

[11] T. Chen, C. Luk, C. Chen, INDUCTWISE: Inductance-Wise Interconnect Simulator and Extractor, IEEE TCAD, vol. 22, No. 7 (Jul. 2003)

[12] J. Jackson, Classical Electrodynamics, 2nd Edition, John Wiley \& Sons, New York (1975)

[13] W. Weeks, L. Wu, M. McAllister, A. Singh, Resistive and inductive skin effect in rectangular conductors, IBM J. Res. Dev., vol. 30, No. 6 (Nov. 1979)
\end{abstract}


DiMES: Multilevel Fast Direct Solver based on Multipole Expansions for Parasitic Extraction of Massively Coupled 3D Microelectronic Structures

\author{
Dipanjan Gope, Indranil Chowdhury, Vikram Jandhyala
}

Department of Electrical Engineering, University of Washington, Seattle, WA

\begin{abstract}
Boundary element methods are being successfully used for modeling parasitic effects in cuttingedge circuit design. The dense system matrix generated therein presents a time and memory bottleneck. Fast iterative solver techniques, developed to address the problem, suffer from convergence issues which become pronounced for large number of right hand sides as is the case for massively coupled systems. In this paper an iteration free solution scheme is presented. The dense matrix is rendered sparse by applying multilevel multipole expansions, and the resultant sparse matrix is solved by a traditional sparse matrix solver. The accuracy and time and memory requirements for the solver are compared against the regular methods. The advantage of the presented method over the corresponding iterative scheme is also demonstrated.
\end{abstract}

Keywords: Parasitics, Multilevel, Multipole, Non-Iterative

\title{
REFERENCES
}

[1] K. Nabors and J. White, "FastCap: a multipole accelerated 3-D capacitance extraction program", IEEE Transactions on Computer-Aided Design of Integrated Circuits and Systems, vol. 10 issue 11 pp.1447-1459, Nov. 1991.

[2] Francis X. Canning and Kevin Rogovin, "Fast direct solution of standard moment-method matrices" IEEE Antennas and Propagation Magazine Vol. 40 No. 3 pp. 15 - 26, June 1998.

[3] D. Gope and V. Jandhyala, "An iteration-free fast multilevel solver for dense method of moment systems", IEEE Proc. on Electrical Performance of Electronic Packaging, pp: 177-180, Oct. 2001.

[4] Shu Yan, Vivek Sarin and Weiping Shi, "Fast capacitance extraction using inexact factorization", IEEE Proc. on Electrical Performance of Electronic Packaging, pp: 285-288, Oct. 2004.

[5] Kenneth Kundert, Sparse Matrix Techniques, in Circuit Analysis, Simulation and Design, Albert Ruehli, (Ed.), North-Holland, 1986. 


\title{
ICCAP: A Linear Time Sparse Transformation and Reordering Algorithm for 3D BEM Capacitance Extraction
}

\author{
Rong Jiang †, Yi-Hao Chang $\neq$, and Charlie Chung-Ping Chen $\dagger$ \\ $\uparrow$ ECE Department, University of Wisconsin, Madison, WI \\ † GIEE \& EE Department, National Taiwan University, Taipei
}

\begin{abstract}
This paper presents an efficient hierarchical 3D capacitance extraction algorithm - ICCAP. Most previous capacitance extraction algorithms introduce intermediate variables to facilitate the hierarchical potential calculation but still preserve the leaf panels as the basis. In this paper, we discover that those intermediate variables are fundamentally much better basis than leaf panels. As a result, we are able to explicitly construct the sparse potential coefficient matrix and solve it with linear memory in linear runtime. Furthermore, the explicit sparse formulation not only enables the usage of preconditioned iterative Krylov subspace methods but also the reordering technique. A new reordering technique is proposed to further reduce over $20 \%$ of memory consumption and runtime in comparison to no reordering techniques applied. Experimental results demonstrate the superior runtime and memory consumption of ICCAP over previous approaches while achieving similar accuracy.

Keywords: Boundary element method, capacitance, parasitic extraction, interconnect, iterative methods

\section{REFERENCES}

[1] K. Nabors and J. White. Fastcap: a multipole accelerated 3d capacitance extraction program. IEEE Trans. on CAD, pages 1447-1459, 1991.

[2] W. Shi, J. Liu, N. Kakani, and T. Yu. A fast hierarchical algorithm for 3-d capacitance extraction. IEEE Trans. On CAD, pages 330-336, 2002.

[3] S. Yan, V. Sarin, andW. Shi. Sparse transformations and preconditioners for hierarchical 3-d capacitance extraction with multiple dielectrics. Proc. DAC, pages 788-793, 2004
\end{abstract}




\title{
Choosing Flows and Methodologies for SoC Design
}

Chair: $\quad$ Dennis C. Wassung, Jr. - Adams Harkness, Inc.

Speakers: $\quad$ Magdy Abadir - Freescale Semiconductor, Inc., Austin, TX Mark Bapst - PrairieComm, Rolling Meadows, IL

Colin Harris - PMC-Sierra, Vancouver, BC

\begin{abstract}
Moving to new semiconductor technology nodes can dramatically impact the business performance of the SoC company, and its age-old design and manufacturing flows and methodologies. It can also significantly affect its choices of suppliers. This session will provide an overview of changing needs and corresponding management decision criteria to make the right choices from a pool of alternate options.
\end{abstract}




\title{
DFM Rules!
}

Chair: $\quad$ Naveed Sherwani - Open-Silicon

Panelists: $\quad$ Alex Alexanian - Ponté Solutions, Mountain View, CA

Harold Lehon - KLA-Tencor, San Jose, CA

Premal Buch - Magma Design Automation, Sunnyvale, CA

Peter Rabkin - Xilinx, San Jose, CA

Carlo Guardiani - PDF Solutions, Desenzano Sul Garda, Italy

Atul Sharan - Clearshape Technologies, Sunnyvale, CA

\begin{abstract}
For sub-100nm processes, predictions are putting initial process yields in the single digits. At the same time, at $130 \mathrm{~nm}$, we saw that two chips designed with the same methodology and same design rules could deliver completely different manufacturing yields.

This panel will discuss the reasons for these phenomena and talk about future trends in DFM that will need to be addressed for success below 100nm.

Keywords: Design for Manufacturability, Yield Optimization
\end{abstract}




\title{
Partitioning-Based Approach to Fast On-Chip Decap Budgeting and Minimization
}

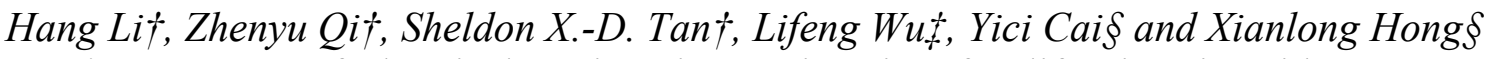 \\ $\dagger$ Department of Electrical Engineering, University of California, Riverside, CA \\ fCadence Design Systems Inc., San Jose, CA \\ $\S$ Department of Computer Science and Technology, Tsinghua University, Beijing, China
}

\begin{abstract}
This paper proposes a fast decoupling capacitance (decap) allocation and budgeting algorithm for both early stage decap estimation and later stage decap minimization in today's VLSI physical design. The new method is based on a sensitivity-based conjugate gradient (CG) approach. But it adopts several new techniques, which significantly improve the efficiency of the optimization process. First, the new approach applies the time-domain merged adjoint network method for fast sensitivity calculation. Second, an efficient search step scheme is proposed to replace the timeconsuming line search phase in conventional conjugate gradient method for decap budget optimization. Third, instead of optimizing an entire large circuit, we partition the circuit into a number of smaller sub-circuits and optimize them separately by exploiting the locality of adding decaps. Experimental results show that the proposed algorithm achieves at least 10X speed-up over the fastest decap allocation method reported so far with similar or even better budget quality and a power grid circuit with about one million nodes can be optimized using the new method in half an hour on the latest Linux workstations.
\end{abstract}

Keywords: Decoupling Capacitor, IR drop, On-Chip Power/Grid Networks

\section{REFERENCES}

[1] S. Bobba, T. Thorp, K. Aingaran, and D. Liu, "IC power distribution challeges," in Proc. Int. Conf. on Computer Aided Design (ICCAD), 2001, pp. 643-650.

[2] H. H. Chen and D. D. Ling, "Power supply noise analysis methodology for deep-submicron VLSI chip design," in Proc. Design Automation Conf. (DAC), 1997, pp. 638-643.

[3] E. Chiprout, "Fast flip-chip power grid analysis via locality and grid shells," in Proc. Int. Conf. on Computer Aided Design (ICCAD), Nov. 2004, pp. 485-488.

[4] E. Chiprout and T. Nguyen, "Power analysis of large interconnect grids with multiple sources using model reduction," in Proc. European Conference on Circuit Theory and Design, Sept. 1999.

[5] J. Fu, Z. Luo, X. Hong, Y. Cai, S. X.-D. Tan, and Z. Pan, "A fast decoupling capacitor budgeting algorithm for robust on-chip power delivery," in Proc. Asia South Pacific Design Automation Conf. (ASPDAC), Jan. 2004, pp. $505-510$.

[6] G. Karypis, R. Aggarwal, and V. K. S. Shekhar, "Multilevel hypergraph partitioning: application in VLSI domain," IEEE Trans. on Very Large Scale Integration (VLSI) Systems, vol. 7, no. 1, pp. 69-79, March 1999.

[7] M. Pant, P. Pant, and D. Wills, "On-chip decoupling capacitor optimization using architectural level current signature prediction," in Proc. IEEE Midwest Symp. Circuits and Systems, 2000, pp. 772-775.

[8] H. F. Qian, S. R. Nassif, and S. S. Sapatnekar, "Random walks in a supply network," in Proc. Design Automation Conf. (DAC), 2003, pp. 93-98.

[9] C. K. S. Zhao, K. Roy, "Decoupling capacitance allocation and its application to power-supply noise-aware floorplanning," IEEE Trans. on Computer-Aided Design of Integrated Circuits and Systems, vol. 21, no. 1, pp. 8192, Jan. 2002.

[10] L. Smith, "Decoupling capacitor calculations for cmos circuits," in Proc. IEEE Topical Meeting of Electrical Performance of Electronic Packaging, 1994, pp. 101-105.

[11] H. Su, S. S. Sapatnekar, and S. R. Nassif, "Optimal decoupling capacitor sizing and placement for standard cell layout designs," IEEE Trans. on Computer-Aided Design of Integrated Circuits and Systems, vol. 22, no. 4, April 2003.

[12] J. Vlach and K. Singhal, Computer Methods for Circuit Analysis and Design. New York, NY: Van Nostrand Reinhold, 1995. 


\title{
Navigating Registers in Placement for Clock Network Minimization
}

Yongqiang Lu ${ }^{a}$, C. N. Sze ${ }^{b}$, Xianlong Hong ${ }^{a}$, Qiang Zhou ${ }^{a}$, Yici Cai ${ }^{a}$, Liang Huang ${ }^{a}$, Jiang Hu $^{b}$

${ }^{a}$ Dept. of Computer Science and Technology, Tsinghua University, Beijing, China

${ }^{\mathrm{b}}$ Dept. of Electrical Engineering, Texas A\&M University, College Station, TX U.S.A.

\begin{abstract}
The progress of VLSI technology is facing two limiting factors: power and variation. Minimizing clock network size can lead to reduced power consumption, less power supply noise, less number of clock buffers and therefore less vulnerability to variations. Previous works on clock network minimization are mostly focused on clock routing and the improvements are often limited by the input register placement. In this work, we propose to navigate registers in cell placement for further clock network size reduction. To solve the conflict between clock network minimization and traditional placement goals, we suggest the following techniques in a quadratic placement framework: (1) Manhattan ring based register guidance; (2) center of gravity constraints for registers; (3) pseudo pin and net; (4) register cluster contraction. These techniques work for both zero skew and prescribed skew designs in both wirelength driven and timing driven placement. Experimental results show that our method can reduce clock net wirelength by $16 \% \sim 33 \%$ with no more than $0.5 \%$ increase on signal net wirelength compared with conventional approaches.
\end{abstract}

Keywords: Clock network, placement, low power, variation tolerance

\section{REFERFENCES}

[1] D. E. Duate, N. Vijaykrishnan and M. J. Irwin, "A clock power model to evaluate impact of architectural and technology optimization," in IEEE TVLSI, 10(6): 844-855, Dec. 2002.

[2] S. Zhao, K. Roy and C.-K. Koh, "Estimation of inductive and resistive switching noise on power supply network in deep sub-micron CMOS circuits," in Proc. IEEE ICCD, pp. 65-72, 2000.

[3] S. Zanella, A. Nardi, A. Neviani, M. Quarantelli, S. Saxena and C. Guardiani, "Analysis of the impact of process variations on clock skew," in IEEE Transactions on Semiconductor Manufacturing, 13(4): 401-407, Nov. 2000.

[4] T.-H. Chao, Y.-C. Hsu, J.-M. Ho, K. D. Boese and A. B. Kahng, "Zero skew clock routing with minimum wirelength," in IEEE Transactions on Circuits \& Systems II: Analog \& Digital Signal Processing, 39 (11): 799-814, Nov. 1992.

[5] J. Cong, A. B. Kahng, C.-K. Koh and C.-W. A. Tsao, "Bounded-skew clock and Steiner routing," in ACM TODAES, 3(3): 341-388, Jul. 1998.

[6] R. Chaturvedi and J. Hu, "A simple yet effective merging scheme for prescribed-skew clock routing," in Proc. IEEE ICCD, pp. 282-287, 2003.

[7] C.-W. A. Tsao and C.-K. Koh, "UST/DME: a clock tree router for general skew constraints," in Proc. IEEE/ACM ICCAD, pp. 400 - 405, 2000.

[8] R. B. Deokar and S. S. Sapatnekar, "A graph-theoretic approach to clock skew optimization”, in Proc. IEEE ISCAS, pp.1.407-1.410, 1994.

[9] J. M. Kleinhans, G. Sigl, F. M. Johannes and K. J. Antreich, "GORDIAN: VLSI placement by quadratic programming and slicing optimization," in IEEE TCAD, 10(3): 356-365, Mar. 1991.

[10] M. Wang and M. Sarrafzadeh, "Congestion minimization during placement," in Proc. ACM ISPD, pp. 145$150,1999$.

[11] W. Hou, X. Hong, W. Wu and Y. Cai, “A path-based timing-driven quadratic placement algorithm," in Proc. IEEE/ACM ASP-DAC, pp.745-748, 2003.

[12] Y. Liu, X. Hong, Y. Cai, W. Wu, “CEP: A clock-driven ECO placement algorithm for standard-cell layout,” in Proc. International Conference on ASIC, pp. 118-121, 2001.

[13] N. Venkateswaran and D. Bhatia, "Clock-skew constrained placement for row based designs," in Proc. IEEE ICCD, pp. 219-220, 1998.

[14] B. Kernighan and S. Lin. "An Efficient Heuristic Procedure for Partitioning of Electrical Circuits”. Bell System Technical Journal, pp. 291-307, 1970.

[15] A. E. Dunlop and B. W. Kernighan, “A procedure for placement of standard-cell VLSI circuits," IEEE TCAD, vol. CAD-4, pp. 92-98, Jan. 1985. 
[16] N. Viswanathan and C.-N Chu, "FastPlace: Efficient Analytical Placement using Cell Shifting, Iterative Local Refinement and a Hybrid Net Model," in Proc. ACM ISPD, pp. 26- 33, 2004. 


\title{
Minimizing Peak Current via Opposite-Phase Clock Tree
}

Yow-Tyng Nieh ${ }^{1}$, Shih-Hsu Huang ${ }^{1}$, Sheng-Yu Hsu ${ }^{2}$

${ }^{1}$ Department of Electronic Engineering, Chung Yuan Christian University, Chung Li, Taiwan, R.O.C.

${ }^{2}$ SoC Technology Center, Industrial Technology Research Institute, Hsin Chu, Taiwan, R.O.C.

\begin{abstract}
Although a lot of research efforts have been made in the minimization of the total power consumption caused by the clock tree, no attention has been paid to the minimization of the peak current caused by the clock tree. In this paper, we propose an opposite-phase scheme for peak current reduction. Our basic idea is to divide the clock buffers at each level of the clock tree into two sets: an half of clock buffers operate at the same phase of the clock source, and another half of clock buffers operate at the opposite phase of the clock source. Consequently, our approach can reduce the peak current of the clock tree nearly 50\%. Experimental data consistently show that our approach works well in practice.
\end{abstract}

Keywords: Physical design, Clock network synthesis, Low power

\section{REFERENCES}

[1] J.L. Neves and E.G. Friedman, "Minimizing Power Dissipation in Non-Zero Skew-Based Clock Distribution Network", Proc. of IEEE International Symposium on Circuits and Systems, vol. 3, pp. 1576-1579, 1995.

[2] J. Pangjun and S.S. Sapatnekar, "Low Power Clock Distribution Using Multiple Voltages and Reduced Swings", IEEE Trans. on Very Large Scale Integration Systems, vol. 10, no. 3, pp. 309-318, 2002.

[3] Q. Wang and S. Roy, "Power Minimization by Clock Root Gating", Proc. of IEEE/ACM Asia and South Pacific Design Automation Conference, pp. 249-254, 2003.

[4] K. Wang and M. Marek-Sadowska, "Buffer Sizing for Clock Power Minimization Subject to General Skew Constraints", Proc. of IEEE/ACM Design Automation Conference, pp. 497-502, 2004.

[5] A. Vittal, H. Ha, F. Brewer and M. Marek-Sadowska, "Clock Skew Optimization for Ground Bounce Control", Proc. Of IEEE/ACM International Conference on Computer Aided Design, pp. 395-399, 1996.

[6] L. Benini, P. Vuillod, A. Bogliolo and G. De Micheli, "Clock Skew Optimization for Peak Current Reduction", Journal of VLSI Signal Processing, vol. 16, pp. 117-130, 1997 
DAC05, pages 186-189

\title{
A Noise-Driven Effective Capacitance Method With Fast Embedded Noise Rule Calculation for Functional Noise Analysis
}

\author{
Haihua Su, David Widiger, Chandramouli Kashyap, Frank Liu, Byron Krauter \\ IBM Corporation, Austin, TX
}

\begin{abstract}
We present a noise-driven effective capacitance method for estimating the combined propagation noise and crosstalk noise. Gate propagation noise rules are efficiently calculated inside the Ceff procedure to determine a linear Thevenin model of the victim driver. A voltage-dependent current source model $[2,6]$ of the driver, along with a load capacitor is analyzed to generate the gate output waveform, from which noise rules are directly extracted. This method removes potential errors introduced in traditional look-up table or fitted-equation based noise rules. The linear driver Thevenin model can then be employed to analyze the propagation noise, while the same Thevenin resistance can be used to analyze the crosstalk noise. The combined coupling and propagation noise can then be estimated using superposition. In this work, we extend the popular timing-driven effective capacitance method into the noise domain. Similar to the effective capacitance method in timing analysis, this technique can successfully separate the nonlinear driver analysis from the linear interconnect analysis. In addition, the linear driver model can significantly ease the task of finding the worst-case peak alignment among all the victim and aggressor noise sources. Experimental results on both RC and RLC nets from industry designs show both accuracy and efficiency compared to SPICE results.
\end{abstract}

Keywords: Effective Capacitance, Glitch Propagation, Noise Analysis

\section{REFERENCES}

[1] R. Arunachalam, F. Dartu, and L. Pileggi. CMOS Gate Delay Models for General RLC Loading. In Proc. ICCAD, pages 224-229, October 1997.

[2] J. F. Croix and D. F. Wong. Blade and Razor: Cell and Interconnect Delay Analysis Using Current-Based Model. In Proc. DAC, pages 386-389, June 2003.

[3] F. Dartu, N. Menezes, and L. Pileggi. Performance Computation for Precharacterized CMOS Gates with RC Loads. IEEE TCAD, pages 1526-1535, May 1996.

[4] R. N. Kanj, T. Lehner, B. Agrawal, and E. Rosenbaum. Noise Characterization of Static CMOS Gates. In Proc. $D A C$, pages 888-893, June 2004.

[5] C. V. Kashyap and B. L. Krauter. A Realizable Driving Point Model for On-Chip Interconnect with Inductance. In Proc. DAC, pages 190-195, June 2000.

[6] I. Keller, K. Tseng, and N. Verghese. A Robust Cell-Level Crosstalk Delay Change Analysis. In Proc. ICCAD, pages 147-154, November 2004.

[7] A. Odabasioglu, M. Celik, and L. T. Pilleggi. PRIMA: Passive Reduced-order Interconnect Macromodeling Algorithm. IEEE TCAD, pages 645-654, Aug 1998.

[8] L. T. Pileggi and R. Rohrer. Asymptotic Waveform Evaluation for Timing Analysis. IEEE TCAD, pages 352366, April 1990.

[9] Synopsys Corp. Library Compiler: Modeling Timing and Power, 2004.

[10] V. Zolotov, D. Blaauw, S. Sirichotiyakul, M. Becer, C. Oh, R. Panda, A. Grinshpon, and R. Levy. Noise Propagation and Failure Criteria for VLSI Designs. In Proc. ICCAD, pages 587-594, November 2002. 


\title{
Constraint-Aware Robustness Insertion for Optimal Noise-Tolerance Enhancement in VLSI Circuits
}

\author{
Chong Zhao, Yi Zhao, Sujit Dey \\ Department of ECE, Univ. of California, San Diego, La Jolla, CA
}

\begin{abstract}
Reliability of nanometer circuits is becoming a major concern in today's VLSI chip design due to interferences from multiple noise sources as well as radiation-induced soft errors. Traditional noise analysis/avoidance and manufacturing testing are no longer sufficient to handle the dynamic interactions between various noise sources and unpredictable operational variations. Therefore, "robustness insertion" has been adopted as the supplementary approach to ensure high circuit reliability through on-line protections. However, the related design overhead is not always acceptable, especially for cost/timing-sensitive designs. In this paper, we present a novel "constraint-aware robustness insertion" methodology protect the sequential elements in digital circuits against various noise effects. Based on a configurable hardening sequential cell design and an efficient sequential cell robustness estimation technique, an optimization algorithm is developed to search for the optimal protection scheme under given timing and area constraints. Experiment results demonstrate that the proposed methodology is able to achieve a high degree of noise-tolerance while keeping the protection cost within limit.
\end{abstract}

Keywords: Nanometer circuits, Robustness calibration, Circuit hardening, Robustness insertion

\section{REFERENCES}

[1] Semiconductor Industry Association, International Technology Roadmap for Semiconductors, 2001

[2] J. Cong, D. Z. Pan, and P. V. Srinivas, "Improved crosstalk modeling for noise constrained interconnect optimization," Proc. ASP-DAC, pp. 373-378, 2001.

[3] Shen Lin, Chang N., "Challenges in power-ground integrity," Proc. ICCAD'01, pp. 651-644, 2001.

[4] J.-J. Liou, A. Krstic, Y.-M. Jiang and K.-T. Cheng, "Modeling, Testing, and Analysis for Delay Defects and Noise Effects in Deep Submicron Devices," IEEE Trans. on Computer-Aided Design of Integrated Circuits and Systems, vol. 22, no. 6, pp. 756-769, Jun. 2003.

[5] Hess C, Stine BE, Weiland LH, Sawada K., "Logic characterization vehicle to determine process variation impact on yield and performance of digital circuits," Intl. Conf. Microelectronic Test Structures, pp. 189-196, 2002.

[6] Peter Hazucha, Christer Svensson, "Cosmic-Ray Soft Error Rate Characterization of a Standard 0.6- m CMOS Process," IEEE Jnl. Solid-State Circuits, vol. 35, no. 10, Oct. 2000.

[7] Anghel, L., Nicolaidis, M., "Cost Reduction and Evaluation of a Temporary Faults Detecting Technique," DATE'00, pp. 591-598, 2000.

[8] E. Dupont, M. Nicolaidis, and P. Rohr, "Embedded Robustness Ips for Transient-Error-Free ICs," IEEE Design \& Test of Computers, pp.56-70, May-June, 2002.

[9] Y. Zhao, S. Dey, "Separate Dual Transistor Register-an Circuit Solution for on-line Testing of Transient Errors in UDSM-IC," Proc.IOLTS, 2003, pp.7-11, 2003.

[10] C.Zhao, X. Bai, S.Dey, "A scalable soft spot analysis methodology for compound noise effects in nano-meter circuits," in Proc. DAC'04, pp. 894-899, 2004.

[11] C.Zhao, X. Bai, S.Dey, "A Static Noise-Impact-Analysis Methodology for Evaluating Transient Error Effects in Digital VLSI Circuits", Research Report, http://esdat.ucsd.edu/ chong/nia.pdf.

[12] T. C. Hu and M. T. Shing, Combinatorial Algorithms, Dover Publications, Inc., pp.111-113, 2002.

[13] T. H. Cormen, C. E. Leiserson, R. L. Rivest and C. Stein, "Introduction to Algorithms", Ch 15, McGraw-Hill, 1990.

[14] Nicolaidis, "Time redundancy based soft-error tolerance to rescue nanometer technologies," Proc. VTS, pp. 8694, 1999.

[15] http://www.tensilica.com/xtensa_overview_handbook.pdf, XtensaTM Microprocessor Overview Handbook, Tensilica Inc, August 2001. 


\title{
Temperature-Aware Resource Allocation and Binding in High-Level Synthesis
}

Rajarshi Mukherjee, Seda Ogrenci Memik, and Gokhan Memik

Department of Electrical and Computer Engineering, Northwestern University, IL, USA

\begin{abstract}
Physical phenomena such as temperature have an increasingly important role in performance and reliability of modern process technologies. This trend will only strengthen with future generations. Attempts to minimize the design effort required for reaching closure in reliability and performance constraints are agreeing on the fact that higher levels of design abstractions need to be made aware of lower level physical phenomena. In this paper, we investigated techniques to incorporate temperature-awareness into high-level synthesis. Specifically, we developed two temperature-aware resource allocation and binding algorithms that aim to minimize the maximum temperature that can be reached by a resource in a design. Such a control scheme will have an impact on the prevention of hot spots, which in turn is one of the major hurdles in front of reliability for future integrated circuits. Our algorithms are able to reduce the maximum attained temperature by any module in a design by up to $19.6^{\circ} \mathrm{C}$ compared to a binding that optimizes switching power.

Keywords: Binding, Temperature, Switching, Leakage
\end{abstract}

\section{REFERENCES}

1. Basu, A., et al. Simultaneous Optimization of Supply and Threshold Voltages for Low-Power and HighPerformance Circuits in the Leakage Dominant Era. Design Automation Conference. 2004.

2. Brooks, D. and M. Martonosi. Dynamic Thermal Management for High-Performance Microprocessors. International Symposium on High-Performance Computer Architecture. 2001.

3. Cao, L., et al., Transient Thermal Management of Portable Electronics using Heat Storage and Dynamic Power Dissipation Control. IEEE Transactions on Components, Packaging, and Manufacturing Technology-Part A, 1998. 21(1): p. 113-123.

4. Chang, J.M. and M. Pedram. Register Allocation and Binding for Low Power. Design Automation Conference. 1995.

5. Chang, J.M. and M. Pedram. Module Assignment for Low Power. European Design Automation Conference. 1996.

6. Chu, C.C.N. and D.F. Wong. A Matrix Synthesis Approach to Thermal Placement. International Symposium on Physical Design. 1997.

7. Cong, J., J. Wei, and Y. Zhang. A Thermal-Driven Floorplanning Algorithm for 3D ICs. International Conference on Computer-Aided Design. 2004.

8. Goldberg, A.V., An Efficient Implementation of a Scaling Minimum-Cost Flow Algorithm. Journal on Algorithms, January 1997. 22(1): p. 1--29.

9. Gunther, S., et al., Managing the Impact of Increasing Microprocessor Power Consumption. Intel Technology Journal, 2001.

10. Huang, W., et al. A Framework for Dynamic Energy Efficiency and Temperature Management. International Symposium on Microarchitecture. 2000.

11. Huang, W., et al. Compact Thermal Modeling for Temperature-Aware Design. Design Automation Conference. 2004.

12. Intel Corp. Intel ${ }^{\circledR}$ Itanium ${ }^{\circledR}$ Processor Overview, www.intel.com/design/itanium/itanium/

13. Krum, A., Thermal Management, in The CRC Handbook of Thermal Engineering, F. Kreith, Editor. 2000, CRC Press: Boca Raton, FL.

14. Lee, C., M. Potkonjak, and W.H. Mangione-Smith. MediaBench: A Tool for Evaluating and Synthesizing Multimedia and Communicatons Systems. International Symposium on Microarchitecture. 1997.

15. Liao, W., F. Lei, and L. He. Microarchitecture Level Power and Thermal Simulation Considering Temperature Dependent Leakage Model. International Symposium on Low Power Electronics and Design. 2003.

16. Lyuh, C. and T. Kim, High-level Synthesis for Low Power Based on Network Flow Method. IEEE Transactions on Very Large Scale Integration Systems, June 2003. 1(3). 
17. Memik, S.O., et al. A Super-Scheduler for Embedded Reconfigurable Systems. International Conference on Computer-Aided Design. 2001.

18. Sabry, M.N. Dynamic Compact Thermal Models: An Overview of Current and Potential Advances. International Workshop on Thermal Investigations of ICs and Systems. 2002.

19. Skadron, K., T. Abdelzaher, and M.R. Stan. Control-Theoretic Techniques and Thermal-RC Modeling for Accurate and Localized Dynamic Thermal Management. Eighth International Symposium on High-Performance Computer Architecture. 2002.

20. Skadron, K., et al. Temperature-aware Microarchitecture. International Symposium on Computer Architecture. 2004.

21. Stanford University Compiler Group The SUIF 2 Compiler System, http://suif.stanford.edu/suif/suif2/

22. Tsai, C. and S. Kang. Standard Cell Placement for Even On-Chip Thermal Distribution. International Symposium on Physical Design. 1999. 


\title{
Leakage Power Optimization With Dual-V th $_{\text {Library In High-Level Synthesis }}$
}

\author{
Xiaoyong Tang ${ }^{1}$, Hai Zhou', Prith Banerjee ${ }^{3}$ \\ ${ }^{1}$ Magma Design Automation, Inc, Santa Clara, CA USA \\ ${ }^{2}$ Northwestern University, Evanston, IL USA \\ ${ }^{3}$ University of Illinois at Chicago, Chicago, IL USA
}

\begin{abstract}
In this paper we address the problem of module selection during high-level synthesis. We present a heuristic algorithm for leakage power optimization based on the maximum weight independent set problem. A dual threshold voltage $\left(\mathrm{V}_{\text {th }}\right)$ technique is used to reduce leakage energy consumption in a data flow graph. Experiments are performed on a data-path dominated test suite of six benchmarks. Our approach achieves an average of $70.9 \%$ leakage power reduction, which is very close to the optimal results from an Integer Linear Programming approach.
\end{abstract}

Keywords : Leakage Power, High-Level Synthesis, Dual- $\mathrm{V}_{\text {th }}$, Optimization

\section{References}

[1] A. Abdollahi, F. Fallah, and M. Pedram, "Leakage Current Reduction in CMOS VLSI Circuits by Input Vector Control," IEEE TVLSI, pp. 140 154, vol. 12, no. 2, 2004.

[2] Artisan Components Inc. www.artisan.com

[3] S. Bobba, and I. Hajj, "Maximum Leakage Power Estimation for CMOS Circuits," Proceedings of the IEEE Alessandro Volta Memorial Workshop on Low Power Design, 1999

[4] D. Chen and M. Sarrafzadeh, “An Exact Algorithm for Low Power Library-Specific Gate Re-Sizing," $25^{\text {th }}$ DAC, 1996.

[5] M. C. Glumbic, Algorithmic Graph Theory and Perfect Graphs, Academic Press, 1980, New York, USA

[6] Dash Company, XPRESS-MP, www.dashoptimization.com

[7] R.X. Gu and M.I.Elmasry, "Power dissipation analysis and optimization of deep submicron CMOS digital circuits," IEEE J. Solid-State Circuits, vol.31, pp. 707-713, May 1996

[8] S. Gupta and F. N. Najm, "Power Macromodeling for High Level Power Estimation,” 34th DAC, 1997.

[9] A. Jones, D. Bagchi, S. Pal, X. Tang, A. Choudhary, P. Banerjee, "PACT HDL: A C Compiler with Power and Performance Optimizations," CASES, France, 2002.

[10] K.S. Khouri, N.K.Jha, "Leakage Power Analysis and Reduction During Behavioral Synthesis," IEEE TVLSI, 2002

[11] K.S. Khouri, G. Lakshminarayana, and N.K. Jha, "IMPACT: A High-Level Synthesis System for Low Power Control-Flow Intensive Circuits,” DATE, France, 1998.

[12] N.Kim, T.Austin, D.Blauuw, T.Mudge, K.Flautner, J.Hu, M. J.Irwin, M.Kandemir, V.Narayanan, "Leakage Current: Moore's Law Meets Static Power,” IEEE Comp. v.36, 2003

[13] A. Raghunathan and N.K.Jha, "Behavioral Synthesis for Low Power", Proceedings of ICCD, 1994.

[14] A. Raghunathan and N.K. Jha, "An ILP formulation for low power based on minimizing switched capacitance during data path allocation,” IEEE Int. Symp. on Cir. \& Sys., 1995.

[15] K. Roy, S. Mukhopadhyay, H. M. Meimand, "Leakage Current Mechanisms and Leakage Reduction Techniques in Deep-Submicrometer CMOS Circuits," Proc. of the IEEE, vol. 91, No.2, February 2003.

[16] K. Roy, S. Prasad, "Low-Power CMOS VLSI Design,” John Wiley and Sons, Inc., 2000.

[17] B.J.Shen, D.L.Scharfetter, P.K.Ko and M.C.Jeng, "BSIM: Berkeley short-channel IGFET model for MOS transisitors," IEEE J. Solid-State Circuits, vol.22, pp.558-565, Aug. 1987

[18] W. T. Shiue, C. Chakrabarti, "Low-Power Scheduling with Resources Operating at Multiple Voltages," IEEE Transactions on Circuits and Systems, vol. 47, No. 6, 2000

[19] A. Srivastava, D. Sylvester, "Minimizing total power by simultaneous Vdd/Vth assignment," Proc. ASPDAC 2003.

[20] Synopsys Inc. "Star-HSPICE Manual," www.synopsys.com

[21] X.Tang, "High-Level Synthesis Algorithms for Low Power ASIC Design," Ph.D. Thesis, 2004.

[22] S. Warshall, A Theorem on Boolean Matrices, Journal of the ACM, 9(1):11-12, 1962 
[23] X.Xi, M.Dunga, J.He, W.Liu, Kcao, X.Jin, J. Ou, M.Chan, A.Nikneja, C.Hu, "BSIM4.3.0 MOSFET Model," http://www-device.eecs.berkeley.edu/ bsim3/bsim4.html

[24] Y. Ye, S. Borkar, and V. De, "A New Technique for Standby Leakage Reduction in High-Performance Circuits," Symposium on VLSI Circuits, 1998, pp. 40-41 


\title{
Incremental Exploration of the Combined Physical and Behavioral Design Space
}

\author{
Zhenyu (Peter) Gu, Jia Wang, Robert P. Dick, Hai Zhou \\ Northwestern University, Evanston, IL, USA
}

\begin{abstract}
Achieving design closure is one of the biggest headaches for modern VLSI designers. This problem is exacerbated by high-level design automation tools that ignore increasingly important factors such as the impact of interconnect on the area and power consumption of integrated circuits. Bringing physical information up into the logic level or even behavioral-level stages of system design is essential to solve this problem. In this paper, we present an incremental floorplanning high-level synthesis system. This system integrates high-level and physical design algorithms to concurrently improve a system's schedule, resource binding, and floorplan, thereby allowing the incremental exploration of the combined behavioral-level and physical-level design space. Compared with previous approaches that repeatedly call loosely coupled floorplanners for physical estimation, this approach has the benefit of efficiency, stability, and better quality of results. For designs containing functional units with non-unity aspect ratios, the average CPU time improved by $369 \%$, the area improved by $14.24 \%$, and power improved by $4 \%$.

Keywords: High-level Synthesis, Incremental, Floorplan
\end{abstract}

\section{REFERENCES}

[1] A. Raghunathan and N. K. Jha, "SCALP: An iterative-improvement-based low-power data path synthesis system," IEEE Trans. Computer-Aided Design of Integrated Circuits and Systems, vol. 16, no. 11, pp. 1260-1277, Nov. 1997.

[2] A. P. Chandrakasan, et al., "Optimizing power using transformations," IEEE Trans. Computer-Aided Design of Integrated Circuits and Systems, vol. 14, no. 1, pp. 12-51, Nov. 1997.

[3] R. S. Martin and J. P. Knight, "Power profiler: Optimizing ASICs power consumption at the behavioral level," in Proc. Design Automation Conf., June 1995.

[4] K. S. Khouri, G. Lakshminarayana, and N. K. Jha, "High-level synthesis of low power control-flow intensive circuits," IEEE Trans. Computer-Aided Design of Integrated Circuits and Systems, vol. 18, no. 12, pp. 1715-1729, Dec. 1999.

[5] D. W. Knapp, "Fasolt: A program for feedback-driven data-path optimization," IEEE Trans. Computer-Aided Design of Integrated Circuits and Systems, vol. 11, no. 6, pp. 677-695, June 1992.

[6] J. P. Weng and A. C. Parker, "3D scheduling: High-level synthesis with floorplanning," in Proc. Design Automation Conf., June 1992.

[7] Y. M. Fang and D. F. Wong, "Simultaneous functional-unit binding and floorplanning," in Proc. Int. Conf. Computer-Aided Design, Nov. 1994.

[8] W. E. Dougherty and D. E. Thomas, "Unifying behavioral synthesis and physical design," in Proc. Design Automation Conf., June 2000.

[9] J. Cong and Z. Pan, "Interconnect performance estimation models for design planning," IEEE Trans. ComputerAided Design of Integrated Circuits and Systems, pp. 739-752, June 2001.

[10] R. Mehra, L. M. Guerra, and J. M. Rabaey, "Low power architecture synthesis and impact of exploiting locality,” J. VLSI Signal Processing, vol. 13, no. 8, pp. 877-888, Aug. 1996.

[11] P. Prabhakaran and P. Banerjee, "Simultaneous scheduling, binding and floorplanning high-level synthesis," in Proc. Int. Conf. VLSI Design, Jan. 1998.

[12] L. Zhong and N. K. Jha, "Interconnect-aware high-level synthesis for low power," in Proc. Int. Conf. Computer-Aided Design, Nov. 2002.

[13] A. Stammermann, et al., "Binding, allocation and floorplanning in low power high-level synthesis," in Proc. Int. Conf. Computer-Aided Design, Nov. 2003.

[14] O. Coudert, et al., "Incremental CAD," in Proc. Int. Conf. Computer-Aided Design, Nov. 2000, pp. 236-244.

[15] H. Zhou and J. Wang, "ACG-Adjacent Constraint Graph for General Floorplans," in Proc. Int. Conf. Computer Design, Oct. 2004.

[16] "NCSU CBL," www.cbl.ncsu.edu/benchmarks/. 
[17] R. P. Dick, D. L. Rhodes, and W. Wolf, “TGFF: Task graphs for free," in Proc. Int. Wkshp. Hardware/Software Co-Design, Mar. 1998, pp. 97-101. 


\title{
Sign Bit Reduction Encoding For Low Power Applications
}

\author{
M. Saneei*, A. Afzali-Kusha*, Z. Navabi** \\ *Low-Power High-Performance Nanosystems Lab., Elec. and Comp. Eng. Dept., \\ University of Tehran, Tehran, Iran \\ **Computer Aided Design Lab., Elec. and Comp. Eng. Dept., University of Tehran, Tehran, Iran
}

\begin{abstract}
This paper proposes a low power technique, called SBR (Sign Bit Reduction) which may reduce the switching activity in multipliers as well as data buses. Utilizing the multipliers based on this scheme, the dynamic power consumption of some digital systems such as digital filters based on CMOS logic system can be reduced considerably compared to those based on 2's complement implementation. To verify the efficacy of the SBR, a 16-bit multiplier was implemented by this scheme. The results for voice data show an average of $29 \%$ to $35 \%$ switching reduction compared to the 2's complement implementation. For 16-bit random data, this scheme decreases the switching of 16-bit multipliers by an average of $21 \%$. Finally, the application of the technique to a 16-bit data bus leads up to $14.5 \%$ switching reduction on average.

Keywords: Switching Activity, Low Power, Signed Multiplier, Bus Encoding, Sing Extension
\end{abstract}

\section{REFERENCES}

[1] Erdogan, T. and Arslan, T. A Coefficient Segmentation Algorithm for Low Power Implementation of FIR filters. Proceedings of the 1999 IEEE International Symposium on Circ. and Sys., Vol. 3 , 30 May-2 June 1999, PP 359362

[2] Ghosh, A. Devadas, S. Keutzer, K. and White, J. Estimation of average switch activity in combinational and sequential circuits. in proc. 29th DAC Conf., June 1992, pp. 253-259

[3] Landman, P. E. and Rabaey, J. M. Architectural Power Analysis: The Dual Bit Type Method. IEEE Trans, on VLSI system, vol. 3, no. 2, June 1995, pp. 173-187

[4] Mou, Z. J. and Duhamel, P. Short-length FIR filters and their use in fast nonrecursive filtering. IEEE Trans. Signal Processing, June 1991, pp. 1322-1323.

[5] Shin, Y. Choi, K. and Chang, Y. H. Narrow Bus Encoding for Low-Power DSP Systems. IEEE Trans. VLSI Syst., vol. 9, no. 5, pp. 656-660, October 2001

[6] Stan, M. R. Burleson, W. P. Bus-Invert Coding for Low-Power I/O. IEEE Trans. VLSI Syst., vol. 3, no. 1, pp. 49-58, March 1995

[7] Stan, M. R. Burleson, W. P. Limited-Weight Codes for Low Power I/O. International Workshop on Low Power Design, Napa, CA, Apr. 1994

[8] Su, C. L. Tsui, C. Y. and Despain, A. M. Low power architecture design and compilation technique for highperformance processors. in Proc. IEEE COMPCON, San Francisco, CA, Feb. 1994, pp. 209-214

[9] Tjarnstrom, R. Power Dissipation Estimate by switch level simulation. Proc. IEEE ISCAS, May 1989, pp. 881884

[10] Yu, Z. Yu, M. L. Azadet, K. and Willson, A. N. Jr. the use of reduced two's complement representation in low power DSP design. IEEE ISCAS, vol. 1, May 2002 pp. I-77 - I-80

[11] Zheng, M. and Albicki, A. Low Power and High Speed Multiplication Design Through Mixed Number Representations. International Conference on Computer Design, October 02-04, 1995, Austin, Texas 
A Watermarking System for IP Protection by a Post Layout Incremental Router

\author{
Tingyuan Nie, Tomoo Kisaka, Masahiko Toyonaga \\ Department of Information Science, Kochi University, 2-5-1 Akebono-cho, Kochi, Japan
}

\begin{abstract}
In this paper, we introduce a new watermarking system for IP protection on post-layout design phase. Firstly the copyright is encrypted by DES (Data Encryption Standard) and then embedded by using an incremental router into the layout design. This watermarking technique uniquely identifies the circuit origin, yet is difficult to be detected or fabricated. The incremental router consists of a rip-up and a special re-router that inserts redundant bends into wires probabilistic. We evaluated the technique on various generated benchmark circuits to validate the completeness of the procedure. The results show it achieves almost $100 \%$ success for embedding with no extra area cost on design performances.
\end{abstract}

Keywords: Intellectual Property Protection (IPP), Post layout design, Incremental router, Watermarking

\title{
REFERENCES
}

[1] Tingyuan Nie, Masahiko Toyonaga, Ken-ichi Shiota, "A watermarking system for VLSI layout using a special router," 2003 Shikoku-section joint convention record of the institutes of electrical and related engineers (in Japanese), pp9-11 2003.

[2] DATA ENCRYPTION STANDARD (DES) http://www.itl.nist.gov/fipspubs/fip46-2.htm.

[3] D. Kirovski, Y.-Y. Hwang, M. Potkonjak and J. Cong "Intellectual Property Protection by Water-marking Combinational Logic Synthesis Solutions," Proc. ACM/IEEE International Conference on Computer Aided Design, San Jose, California, pp. 194-198, November 1998.

[4] A.B. Kahng, J.Lach, W. H. M-Smith, S.Mantik, I. L. Markov, M.Potkonjak, P. Tucker, H.Wang, G.Wolfe, "Constraint-Based Water-marking Techniques for Design IP Protection," IEEE Transactions on Computer-Aided Design of Integrated circuits and Systems, VOL. 20, NO. 10, pp. 1236-1252, October 2001.

[5] S.H. Kwok, C.C. Yang, K.Y. Tam, "Water-mark Design Pattern for Intellectual Property Protection in Electronic Commerce Applications", 33rd Hawaii International Conference on System Sciences-Volume 6, pp.6038, January $0407,2000$.

[6] John Lach, William H. Mangione-Smith, Miodrag Potkonjak, "Fingerprinting Techniques for FieldProgrammable Gate Array Intellectual Property Protection," IEEE Transactions on Computer-Aided Design of Integrated circuits and Systems, VOL. 20, NO. 10, pp. 1253-1261, October 2001.

[7] A. B. Kahng, J. Lach, W. H. Mangione-Smith, S. Mantik, I. L. Markov, M. Potkonjak, P. Tucker, H. Wang and G. Wolfe, "Watermarking Techniques for Intellectual Property Protection", $35^{\text {th }}$ Design Automation Conference, pp.776-781, June 1998.

[8] Andrew B. Kahng, Stefanus Mantik, Igor L. Markov, Miodrag Potkonjak, Paul Tucker†, Huijuan Wang and Gregory Wolfe, "Robust IP Water-marking Methodologies for Physical design", $35^{\text {th }}$ Conference on Design Automation Conference (DAC'98), pp. 782-787, June 15 19, 1998.

[9] Greg Wolfe, Jennifer L. Wong, and Miodrag Potkonjak, "Watermarking Graph Partitioning Solutions," 38th Conference on Design Automation (DAC'01), pp. 486-489, June 18 22, 2001.

[10] Miodrag Potkonjak, "Water-marking While Preserving The Critical Path", 37th Conference on Design Automation (DAC'00), pp. 108-111, June 05-09, 2000.

[11] Amr T. Abdel-Hamid, Sofi 'ene Tahar, El Mostapha Aboulhamid, "IP Water-marking Techniques: Survey and Comparison", The $3^{\text {rd }}$ IEEE International Workshop on System-on-Chip for Real-Time Applications (IWSOC'03), pp. 60, June 30 - July $02,2003$. 


\title{
A Side-Channel Leakage Free Coprocessor IC in 0.18 $\mu \mathrm{m}$ CMOS for Embedded AES-based Cryptographic and Biometric Processing
}

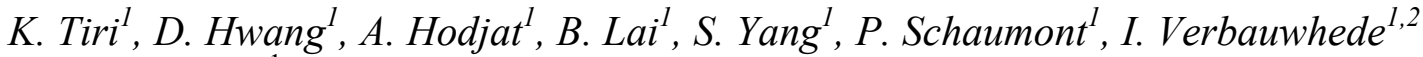 \\ ${ }^{1}$ Electrical Engineering Dept., UC Los Angeles, USA \\ ${ }^{2}$ Dept. ESAT/SCD-COSIC, K.U.Leuven, Belgium
}

\begin{abstract}
Security ICs are vulnerable to side-channel attacks (SCAs) that find the secret key by monitoring the power consumption and other information that is leaked by the switching behavior of digital CMOS gates. This paper describes a side-channel attack resistant coprocessor IC and its design techniques. The IC has been fabricated in $0.18 \mu \mathrm{m}$ CMOS. The coprocessor, which is used for embedded cryptographic and biometric processing, consists of four components: an Advanced Encryption Standard (AES) based cryptographic engine, a fingerprint-matching oracle, a template storage, and an interface unit. Two functionally identical coprocessors have been fabricated on the same die. The first, 'secure', coprocessor is implemented using a logic style called Wave Dynamic Digital Logic (WDDL) and a layout technique called differential routing. The second, 'insecure', coprocessor is implemented using regular standard cells and regular routing techniques. Measurement-based experimental results show that a differential power analysis (DPA) attack on the insecure coprocessor requires only 8,000 acquisitions to disclose the entire $128 \mathrm{~b}$ secret key. The same attack on the secure coprocessor still does not disclose the entire secret key at 1,500,000 acquisitions. This improvement in DPA resistance of at least 2 orders of magnitude makes the attack de facto infeasible. The required number of measurements is larger than the lifetime of the secret key in most practical systems.

Keywords: Countermeasure, Side-Channel Attack, Differential Power Analysis, Encryption, Smart Card, Security IC

\section{REFERENCES}

[1] M. Renaudin, F. Bouesse, P. Proust, J. Tual, L. Sourgen and F. Germain, "High Security Smart-cards," DATE, pp. 228-233, 2004.

[2] P. Kocher, R. Lee, G. McGraw, A. Raghunathan and S. Ravi, "Security as a New Dimension in Embedded System Design," DAC, pp. 753-760, 2004.

[3] E. Oswald, S. Mangard and N. Pramstaller, "Secure and Efficient Masking of AES - A Mission Impossible?," IACR Cryptology ePrint, 2004.

[4] K. Tiri and I. Verbauwhede, "A Logic Level Design Methodology for a Secure DPA Resistant ASIC or FPGA Implementation,” DATE, pp. 246-251, 2004.

[5] K. Tiri and I. Verbauwhede, "A Digital Design Flow for Secure Integrated Circuits," submitted IEEE TCAD.

[6] K. Tiri and I. Verbauwhede, "Place and Route for Secure Standard Cell Design," CARDIS, pp. 143-158, 2004.

[7] D. Hwang, P. Schaumont, K. Tiri and I. Verbauwhede, "Making Embedded Systems Secure," accepted IEEE Security \& Privacy Magazine.

[8] S. Moore, R. Anderson, R. Mullins, G. Taylor and J. Fournier, "Balanced self-checking asynchronous logic for smart card applications" Microprocessors and Microsystems 27.9, pp. 421-430, 2003.

[9] N. Pramstaller, F. Gürkaynak, S. Häne, H. Kaeslin, N. Felber, and W. Fichtner, "Towards an AES Crypto-chip Resistant to Differential Power Analysis”, ESSCIRC, pp. 307-310, 2004.
\end{abstract}




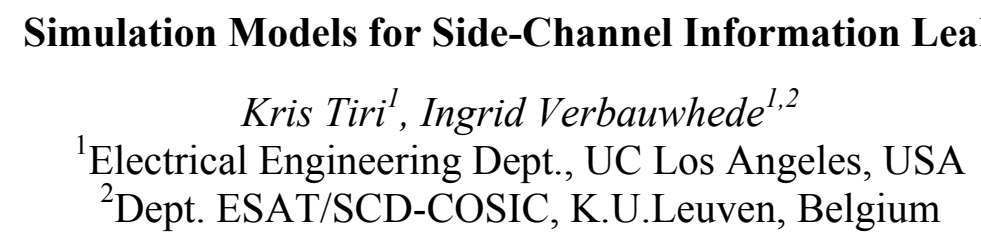

\begin{abstract}
Small, embedded integrated circuits (ICs) such as smart cards are vulnerable to so-called sidechannel attacks (SCAs). The attacker can gain information by monitoring the power consumption, execution time, electromagnetic radiation and other information that is leaked by the switching behavior of digital CMOS gates. Ever since power attacks have been introduced in 1999, many countermeasures have been proposed. Often a significant increase in security has been touted. We will show that in order to assess the effectiveness of a countermeasure, a correct simulation model of the side-channel information leaks is vital. We will show that seemingly correct approximations can lead to completely flawed results.
\end{abstract}

Keywords: Simulation Model, Countermeasure, Side-Channel Attack, Differential Power Analysis, Encryption, Smart Card, Security IC

\title{
REFERENCES
}

[1] M. Renaudin, F. Bouesse, P. Proust, J. Tual, L. Sourgen and F. Germain, "High Security Smartcards", DATE, pp. 228-232, February 2004.

[2] P. Kocher, R. Lee, G. McGraw, A. Raghunathan, and S. Ravi, "Security as a New Dimension in Embedded System Design", DAC, pp.735-760, June 2004.

[3] A. Shamir, and E. Tromer, "Acoustic cryptanalysis", http://www.wisdom.weizmann.ac.il/ tromer/acoustic/, 2004.

[4] B. Schneier, "A Hardware DES Cracker", Crypto-Gram Newsletter, http://www.schneier.com/crypto-gram9808.html\#descracker, August 1998.

[5] K. Tiri, D. Hwang, A. Hodjat, B. Lai, S. Yang, P. Schaumont, and I. Verbauwhede, "A Side-Channel Leakage Free Coprocessor IC in $0.18 \mu \mathrm{m}$ CMOS for Embedded AES-based Cryptographic and Biometric Processing", DAC, June 2005.

[6] P. Kocher, J. Jaffe and B. Jun, "Differential Power Analysis", CRYPTO, LNCS 1666, pp. 388-397, August 1999.

[7] T. Messerges, E. Dabbish, and R. Sloan, "Examining smartcard security under the threat of power analysis attacks", IEEE TC, Vol. 51, Issue: 5, pp. 541-552, May 2002.

[8] C. Clavier, J. Coron, and N. Dabbous, "Differential Power Analysis in the Presence of Hardware Countermeasures", CHES, LNCS 1965, pp. 252-263, August 2000.

[9] A. Shamir, "Protecting Smart Cards from Passive Power Analysis with Detached Power Supplies", CHES, LNCS 1965, pp. 71-77, August 2000.

[10] N. Pramstaller, F. Gürkaynak, S. Häne, H. Kaeslin, N. Felber, and W. Fichtner, "Towards an AES Crypto-chip Resistant to Differential Power Analysis”, ESSCIRC, pp. 307-310, September 2004.

[11] E. Oswald, S. Mangard and N. Pramstaller, "Secure and Efficient Masking of AES - A Mission Impossible?", Report 2004/134 in IACR Cryptology ePrint Archive, June 2004

[12] S. Mangard, T. Popp, and B. Gammel, "Side-Channel Leakage of Masked CMOS Gates", CT-RSA, Feb. 2005.

[13] K. Tiri, and I. Verbauwhede, "Place and Route for Secure Standard Cell Design", CARDIS, pp. 143-158, August 2004.

[14] L. Benini, A. Macii, E. Macii, E. Omerbegovic, F. Pro, et al., "Energy-aware design techniques for differential power analysis protection", DAC, pp. 36-41, June 2003.

[15] H. Saputra, N. Vijaykrishnan, M. Kandemir, M. Irwin, R. Brooks, S. Kim, et al., "Masking the energy behavior of DES encryption", DATE, pp. 84-89, March 2003.

[16] C. Gebotys "Design of secure cryptography against the threat of power-attacks in DSP-embedded processors", ACM TECS, Vol. 3, Issue 1, pp. 92-113, February 2004.

[17] J. Coron, P. Kocher, and D. Naccache, "Statistics and Secret Leakage", FC, LNCS 1962, pp. 157-173, Feb. 2000. 
[18] J. Coron, "Resistance against differential power analysis for elliptic curve cryptosystems," CHES, LNCS 1717, pp. 292-302, August 1999.

[19] K. Tiri and I. Verbauwhede, “A Logic Level Design Methodology for a Secure DPA Resistant ASIC or FPGA Implementation", DATE, pp. 246-251, February 2004.

[20] S. Mangard, "Hardware Countermeasures Against DPA - A Statistical Analysis of Their Effectiveness", CTRSA, LNCS 2964, pp. 222 - 235, February 2004.

[21] F. Mace, F. Standaert, I. Hassoune, J. Legat and J. Quisquater, “A Dynamic Current Mode Logic to Counteract Power Analysis Attacks", DCIS, November 2004 


\title{
A Pattern Matching Coprocessor for Network Security
}

Young H. Cho and William H. Mangione-Smith

University of California, Los Angeles, Department of Electrical Engineering

Los Angeles, California

\begin{abstract}
It has been estimated that computer network worms and virus caused the loss of over $\$ 55 \mathrm{~B}$ in 2003. Network security system use techniques such as deep packet inspection to detect the harmful packets. While software intrusion detection system running on general purpose processors can be up-dated in response to new attacks. They lack the processing power to monitor gigabit networks. We present a high performance pattern matching co-processor architecture that can be used to monitor and identify a large number of intrusion signature. The design consists of a bank of pattern matchers that are used to implement a highly concurrent filter. The pattern matchers can be programmed to match multiple patterns of various lengths, and are able to leverage the existing databases of threat signatures. We have been able to program the filters to match all the payload patterns defined in the widely used Snort network intrusion detection system at a rate above $7 \mathrm{Gbps}$, with memory space left to accommodate threat signatures that become available in the future.
\end{abstract}

Keywords: Network security, Intrusion, Pattern matching, Pattern search, Snort

\section{REFERENCES}

[1] Seda O. Memik Gokhan Memik and William H. Mangione-Smith, "Design and Analysis of a Layer Seven Network Processor Accelerator Using Reconfigurable Logic," in IEEE Symposium on Field-Programmable Custom Computing Machines, Napa Valley, CA, April 2002, IEEE.

[2] Young H. Cho, Shiva Navab, and William H. Mangione-Smith, "Specialized Hardware for Deep Network Packet Filtering," in 12th Conference on Field Programmable Logic and Applications, Montpellier, France, September 2002, pp. 452-461, Springer-Verlag.

[3] Young H. Cho and William H. Mangione-Smith, "Deep Packet Filter with Dedicated Logic and Read Only Memories," in IEEE Symposium on Field-Programmable Custom Computing Machines, Napa Valley, CA, April 2004, IEEE.

[4] Neil Desi, "Increasing Performance in High Speed NIDS: A look at Snort's Internals," Feb 2002.

[5] David Watson, Matthew Smart, G. Robert Malan, and Farnam Jahanian, "Protocol Scrubbing: Network Security through Transparent Flow Modification," in IEEE/ACM Transactions on Networking. April 2004, ACM Press.

[6] R. Sidhu and V. K. Prasanna, "Fast Regular Expression Matching using FPGAs," in IEEE Symposium on FieldProgrammable Custom Computing Machines, Napa Valley, CA, April 2001, IEEE.

[7] R. Franklin, D. Carver, and B. L. Hutchings, "Assisting Network Intrusion Detection with Reconfigurable Hardware," in Proceedings of the IEEE Symposium on FPGA's for Custom Computing Machines, Napa Valley, CA, April 2002, IEEE.

[8] Ioannis Sourdis and Dionisios Pnevmatikatos, "Fast, Large-Scale String Match for a 10Gbps FPGA-based Network Intrusion Detection System," in $13^{\text {th }}$ Conference on Field Programmable Logic and Applications, Lisbon, Portugal, September 2003, Springer-Verlag.

[9] Christopher R. Clark and David E. Schimmel, "Scalable Parallel Pattern-Matching on High-Speed Networks," in IEEE Symposium on Field-Programmable Custom Computing Machines, Napa Valley, CA, April 2004, IEEE.

[10] Zachary K. Baker and Viktor K. Prasanna, "A Methodology for Synthesis of Efficient Intrusion Detection Systems on FPGAs," in IEEE Symposium on Field-Programmable Custom Computing Machines, Napa Valley, CA, April 2004, IEEE.

[11] M. Gokhale, D. Dubois, A. Dubois, M. Boorman, S. Poole, and V. Hogsett, "Granidt: Towards Gigabit Rate Network Intrusion Detection Technology," in 12th Conference on Field Programmable Logic and Applications, Montpellier, France, September 2002, pp. 404-413, Springer-Verlag.

[12] J.W. Lockwood, J. Moscola, M. Kulig, D. Reddick, and T. Brooks, "Internet Worm and Virus Protection in Dynamically Reconfigurable Hardware," in Military and Aerospace Programmable Logic Device (MAPLD), Washington DC, September 2003, NASA Office of Logic Design. 
[13] Young H. Cho and William H. Mangione-Smith, "Programmable Hardware for Deep Packet Filtering on a Large Signature Set," in First IBM Watson P=ac2 Conference, Yorktown, NY, October 2004, IBM.

[14] Alfred V. Aho and Margaret J. Corasick, "Efficient String Matching: An Aid to Bibliographic Search," in Communications of the ACM. June 1975, pp. 333-340, ACM Press. 


\title{
High Performance Encryption Cores for 3G Networks
}

Tomás Balderas-Contreras, René Cumplido

Instituto Nacional de Astrofísica, Óptica y Electrónica, Tonantzintla, Puebla, MEXICO

\begin{abstract}
This paper presents two novel and high performance hardware architectures, implemented in FPGA technology, for the KASUMI block cipher; this algorithm lies at the core of the confidentiality and integrity algorithms defined for the Universal Mobile Telecommunication System (UMTS) standard. The first proposal is a pipelined design and the second implements an iterative approach. The throughput for these architectures turn out to be higher than the throughput achieved by other proposals.
\end{abstract}

Keywords: 3G, UMTS Security Architecture, KASUMI, FPGA

\section{REFERENCES}

[1] 3rd Generation Partnership Program. Document 2: KASUMI Specification. Technical Specification 35.202. Release 5. Version 5.0.0.

[2] H. Kim, Y. Choi, M. Kim and H. Ryu. Hardware Implementation of the 3GPP KASUMI Crypto Algorithm. In ITC-CSCC-2002 Conference Proceedings. 2002.

[3] P. Kitsos, M. D. Galanis and O. Koufopavlou. High-Speed Hardware Implementations of the KASUMI Block Cipher. In ISCAS'04 Conference Proceedings. 2004.

[4] K. Marinis, N. K. Moshopoulos, F. Karoubalis and K. Z. Pekmestzi. On the Hardware Implementation of the 3GPP Confidentiality and Integrity Algorithms, In $4^{\text {th }}$ ISC 2001 Conference Proceedings. 2001.

[5] A. Satoh and S. Morioka. Small and High-Speed Hardware Architectures for the 3GPP Standard Cipher KASUMI. In 5th ISC 2002 Conference Proceedings. 2002. 


\title{
Efficient Fingerprint-based User Authentication for Embedded Systems
}

Pallav Gupta†, Srivaths Ravił, Anand Raghunathan $\neq$, Niraj K. Jha†

$\dagger$ Dept. of Electrical Engineering, Princeton University, Princeton, NJ, USA

f NEC Laboratories America Inc., Princeton, NJ, USA

\begin{abstract}
User authentication, which refers to the process of verifying the identity of a user, is becoming an important security requirement in various embedded systems. While conventional solutions for user authentication have relied on password-based mechanisms, they are increasingly being replaced by biometric technologies such as fingerprint, face, and voice recognition, which are known to provide higher levels of security for user authentication. This paper investigates the problem of supporting efficient fingerprint-based user authentication in embedded systems. For improving the performance of fingerprint-based authentication, we propose hardware/software enhancements that include a generic set of custom instruction extensions to an embedded processor's instruction set architecture, a memory-aware software re-design, and fixed-point arithmetic. We believe that the custom instruction set extensions proposed in this work are generic enough to speed up many fingerprint matching algorithms and even other biometric algorithms. Our experiments with an open-source, high-fidelity fingerprint authentication algorithm and a testbed featuring a commercial extensible processor show that performance is improved by a factor of $10.4 \mathrm{X}$ when using the proposed enhancements, while incurring modest overheads.
\end{abstract}

Keywords: User authentication, fingerprint, extensible processors

\section{REFERENCES}

[1] R. York, A New Foundation for CPU Systems Security. ARM Limited, 2003.

[2] P. Kocher, R. B. Lee, G. McGraw, A. Raghunathan, and S. Ravi, "Security as a new dimension in embedded system design," in Proc. Design Automation Conf., June 2004, pp. 753-760.

[3] I. Armstrong, "Passwords exposed: Users are the weakest link," in http://www.scmagazine.com, June 2003.

[4] A. Jain, R. Bolle, and S. Pankanti, Eds., Biometrics: Personal Identification in Networked Society. Boston, MA: Kluwer Academic, 2002.

[5] L. Hong, Y. Wan, and A. K. Jain, "Fingerprint image enhancement: Algorithm and performance evaluation," IEEE Trans. Pattern Anal. Machine Intell., vol. 20, no. 8, pp. 777-789, Aug. 1998.

[6] "FVS website." http://fvs.sourceforge.net

[7] S. Yang and I. M. Verbauwhede, "A secure fingerprint matching technique," in Proc. Wkshp. Biometrics Applications \& Methods, Nov. 2003, pp. 89-94.

[8] D. Maltoni, D. Maio, A. K. Jain, and S. Prabhakar, Handbook of Fingerprint Recognition. New York, NY: Springer, 2003.

[9] T. Y. Tang, Y. S. Moon, and K. C. Chan, "Efficient implementation of fingerprint verification for embedded systems using fixed-point arithmetic," in Proc. Symp. Applied Computing, Mar. 2004, pp. 821-825.

[10] P. Schaumont and I. Verbauwhede, “Thumbpod puts security under your thumb," Xilinx Xcell J., 2003.

[11] Xtensa Application Specific Microprocessor Solutions - Overview Handbook. Tensilica Inc., 2001. http://www.tensilica.com

[12] "Authentec website." http://www.authentec.com

[13] R. Andraka, "A survey of CORDIC algorithms for FPGAs," in Proc. Int. Symp. Field-Programmable Gate Arrays, Feb. 1998, pp. 191-200. 


\title{
Approximate VCCs: A New Characterization of Multimedia Workloads for System-level MpSoC Design
}

\author{
Yanhong Liu, Samarjit Chakraborty, Wei Tsang Ooi \\ Department of Computer Science, National University of Singapore
}

\begin{abstract}
System-level design methods specifically targeted towards multimedia applications have recently received a lot of attention. Multimedia workloads are known to have a high degree of variability. Therefore, designs based on a worst-case analysis of such workloads tend of be overly pessimistic. We address this issue by introducing a new concept called approximate variability characterization curves (or Approximate VCCs), to characterize the "average-case" behavior of multimedia workloads in a parameterized fashion. Since most multimedia applications only have soft real-time constraints, it is often possible to tolerate a small amount of performance degradation. By allowing such small degradations in the performance, large amounts of resource savings are possible. The concept of Approximate VCCs that we present in this paper allows a designer to quantitatively account for the performance degradation and the associated resource savings. We illustrate this using two typical system design cases.
\end{abstract}

Keywords: Multimedia, Workload, System-level design

\section{REFERENCES}

[1] S. Ayyorgun and R. L. Cruz. A composable service model with loss and a scheduling algorithm. In INFOCOM, Hong Kong, China, March 2004.

[2] S. Ayyorgun and R. L. Cruz. A service-curve model with loss and a multiplexing problem. In ICDCS, Tokyo, Japan, March 2004.

[3] R. Boorstyn, A. Burchard, J. Leibeherr, and C. Oottamakorn. Statistical service assurances for traffic scheduling algorithms. IEEE Journal on Selected Areas in Communications, 18(13):2651-2664, 2000.

[4] J.-Y. Le Boudec and P. Thiran. Network Calculus - A Theory of Deterministic Queuing Systems for the Internet. LNCS 2050, 2001.

[5] W. Chase and F. Bown. General Statistics. John Wiley \& Sons, 1997.

[6] F. Ciucu, A. Burchard, and J. Liebeherr. A network service curve approach for the stochastic analysis of networks. In ACM Sigmetrics, 2005.

[7] R. Cruz. A calculus for network delay, Parts 1 \& 2. IEEE Transactions on Information Theory, 37(1), 1991.

[8] C. A. Gonzales, H. Yeo, and C. J. Kuo. Requirements for motion-estimation search range in MPEG-2 coded video. IBM Journal of Research and Development, 43(4), 1999.

[9] Y. Liu, A. Maxiaguine, S. Chakraborty, and W. T. Ooi. Processor frequency selection for SoC platforms for multimedia applications. In RTSS, Lisbon, Portugal, December 2004.

[10] A. Maxiaguine, Y. Zhu, S. Chakraborty, and W.-F. Wong. Tuning SoC platforms for multimedia processing: Identifying limits and tradeoffs. In CODES+ISSS, Stockholm, Sweden, September 2004.

[11] A. Nandi and R. Marculescu. System-level power/performance analysis for embedded systems design. In $D A C$, Las Vegas, Nevada, USA, June 2001.

[12] M.J. Rutten, J.T.J. van Eijndhoven, E.G.T. Jaspers, P. van der Wolf, O.P. Gangwal, and A. Timmer. A heterogeneous multiprocessor architecture for flexible media processing. IEEE Design \& Test of Computers, 19(4):39-50, July-August 2002.

[13] G. Varatkar and R. Marculescu. On-chip traffic modeling and synthesis for MPEG-2 video applications. IEEE Transactions on VLSI, 12(1):108-119, January 2004.

[14] W. Yuan and K. Nahrstedt. Energy-efficient soft real-time CPU scheduling for mobile multimedia systems. In SOSP, NY, USA, October 2003. 


\title{
Modular Domain-Specific Implementation and Exploration Framework for Embedded Software Platforms
}

\author{
Christian Sauer, Matthias Gries, Sören Sonntag \\ Infineon Technologies, Corporate Research, Munich, Germany
}

\begin{abstract}
This paper focuses on designing network processing software for embedded processors. Our design flow $C R A C C$ represents an efficient path to implementation based on a modular application description, while avoiding much of the overhead of existing component-based techniques. We illustrate results for a real-world application implementing a full IP-based DSL Access Multiplexer (IP-DSLAM) system. We quantify overhead and optimization potential incurred by our modular implementation. We also point out how CRACC can be deployed for HW-SW partitioning and design space exploration.

Keywords: Software Development, Programmable Platforms, Design Space Exploration, DSLAM, Network Processing
\end{abstract}

\section{REFERENCES}

[1] A. Bender: MILP Based Task Mapping for Heterogeneous Multiprocessor Systems, EURO-DAC, 1996

[2] B. Chen, R. Morris: Flexible Control of Parallelism in a Multiprocessor PC Router, USENIX, June 2001

[3] K. Crozier: A C-Based Programming Language for Multiprocessor Network SoC Architectures, Network Processor Design, vol. 2, Morgan Kaufmann, Nov. 2003

[4] A.I. Holub: $C+C++$ : Programming With Objects in $C$ and $C++$, McGraw-Hill, 1991

[5] E. Kohler, R. Morris, B. Chen, J. Jannotti, M. F. Kaashoek: The Click Modular Router, ACM Transactions on Computer Systems, 18(3), Aug. 2000

[6] C. Kulkarni, G. Brebner, G. Schelle: Mapping a Domain Specific Language to a Platform FPGA, DAC, 2004

[7] P. Marwedel: Embedded Software: How to Make it Efficient? Digital System Design, Euromicro, Sept. 2002

[8] P. Paulin, C. Pilkington, E. Bensoudane: StepNP: A System-Level Exploration Platform for Network Processors, IEEE Design and Test of Computers, 19(6), 2002

[9] P. Paulin, C. Pilkington, M. Langevin, E. Bensoudane, G. Nicolescu: Parallel Programming Models for a Multiprocessor SoC Platform Applied to High-Speed Traffic Management, CODES+ISSS, 2004

[10] C. Sauer, M. Gries, S. Sonntag: Modular Reference Implementation of an IP-DSLAM, ISCC, June 2005

[11] N. Shah, W. Plishker, K. Keutzer: NP-Click: A Programming Model for the Intel IXP1200, Network Processor Design, vol. 2, Morgan Kaufmann, Nov. 2003

[12] I. Sommerville: Software Engineering, International Computer Science Series, 7th edition, Addison Wesley, 2004

[13] L. Thiele, S. Chakraborty, M. Gries, S. Künzli: A Framework for Evaluating Design Tradeoffs in Packet Processing Architectures, Design Automation Conference (DAC), 2002 


\author{
DAC05, pages 260-265 \\ Simulation Based Deadlock Analysis for System Level Designs \\ Xi Chen ${ }^{l}$, Abhijit Davare ${ }^{2}$, Harry Hsieh $^{1}$, Alberto Sangiovanni-Vincentelli ${ }^{2}$, Yosinori Watanabe ${ }^{3}$ \\ ${ }^{1}$ University of California, Riverside, CA \\ ${ }^{2}$ University of California, Berkeley, CA \\ ${ }^{3}$ Cadence Berkeley Laboratories, Berkeley, CA
}

\begin{abstract}
In the design of highly complex, heterogeneous, and concurrent systems, deadlock detection and resolution remains an important issue. In this paper, we systematically analyze the synchronization dependencies in concurrent systems modeled in the Metropolis design environment, where system functions, high level architectures and function-architecture mappings can be modeled and simulated. We propose a data structure called the dynamic synchronization dependency graph, which captures the runtime (blocking) dependencies. A loopdetection algorithm is then used to detect deadlocks and help designers quickly isolate and identify modeling errors that cause the deadlock problems. We demonstrate our approach through a real world design example, which is a complex functional model for video processing and a high level model of function-architecture mapping.

Keywords: simulation, deadlock, synchronization, cyclic dependency, system level, Metropolis
\end{abstract}

\title{
REFERENCES
}

[1] http://www.eda.org/vfv, 2003.

[2] F. Balarin, Y. Watanabe, J. Burch, L. Lavagno, R. Passerone, and A. Sangiovanni-Vincentelli. Constraints specification at higher levels of abstraction. In Proceedings of International Workshop on High Level Design Validation and Test, Nov. 2001.

[3] F. Balarin, Y. Watanabe, H. Hsieh, L. Lavagno, C. Passerone, and A. Sangiovanni-Vincentelli. Metropolis: an Integrated Electronic System Design Environment. IEEE Computer, 36(4):45- 52, Apr. 2003.

[4] A. Charlesworth. The multiway rendezvous. ACM Transactions on Programming Languages and Systems, 9(3):350-366, 1987.

[5] E. G. Coffman, M. Elphick, and A. Shoshani. System deadlocks. ACM Computing Surveys, 3(2):67-78, 1971.

[6] O. Gangwal, A. Nieuwland, and P. Lippens. A scalable and flexible data synchronization scheme for embedded hw-sw shared-memory systems. In Proceedings of International Symposium on System Synthesis, Oct. 2001.

[7] P. Godefroid and D. Pirottin. Refining dependencies improves partial-order verification methods. In Proceedings of the 5th Conference on Computer Aided Verification, volume 697 of Lecture Notes in Computer Science, pages 438-449. Springer-Verlag, June 1993.

[8] A. N. Habermann. Prevention of system deadlocks. Communications of the ACM, 12(7):373-377, 1969.

[9] C. A. R. Hoare. Communicating sequential processes. Communications of the ACM, 21(8):666-677, 1978.

[10] G. J. Holzmann. The model checker SPIN. IEEE Trans. on Software Engineering, 23(5):279-258, May 1997.

[11] K. Keutzer, S. Malik, A. R. Newton, J. Rabaey, and A. Sangiovanni-Vincentelli. System level design: orthogonalization of concerns and platform-based design. IEEE Transactions on Computer-Aided Design, 19(12):1523-1543, Dec. 2000.

[12] E. Knapp. Deadlock detection in distributed databases. ACM Computing Surveys, 19(4):303-328, 1987.

[13] M. Krishnamurthi, A. Basavatia, and S. Thallikar. Deadlock detection and resolution in simulation models. In Proceedings of the 26th Conference on Winter Simulation, pages 708-715. Society for Computer Simulation International, 1994.

[14] Z. Manna and A. Pnueli. The temporal logic of reactive and concurrent systems: Specification. SpringerVerlag, 1992.

[15] K. McMillan. Symbolic Model Checking. Kluwer Academic Publishers, 1993.

[16] J. L. Peterson and A. Silbershatz. Operating System Concepts. Addison-Wesley, 1983.

[17] M. Sfinghal. Deadlock detection in distributed systems. IEEE Computer, 22(11):37-48, 1989. 


\title{
Fault and Energy-Aware Communication Mapping with Guaranteed Latency for Applications Implemented on NoC
}

\author{
Sorin Manolache, Petru Eles, Zebo Peng \\ Linköping University, Sweden
}

\begin{abstract}
As feature sizes shrink, transient failures of on-chip network links become a critical problem. At the same time, many applications require guarantees on both message arrival probability and response time. We address the problem of transient link failures by means of temporally and spatially redundant transmission of messages, such that designer-imposed message arrival probabilities are guaranteed. Response time minimisation is achieved by a heuristic that statically assigns multiple copies of each message to network links, intelligently combining temporal and spatial redundancy. Concerns regarding energy consumption are addressed in two ways. Firstly, we reduce the total amount of transmitted messages, and, secondly, we minimise the application response time such that the resulted time slack can be exploited for energy savings through voltage reduction. The advantages of the proposed approach are guaranteed message arrival probability and guaranteed worst case application response time.
\end{abstract}

General Terms: Algorithms, Performance

\section{REFERENCES}

[1] A. Andrei, M. Schmitz, P. Eles, Z. Peng, and B. Al-Hashimi. Simultaneous communication and processor voltage scaling for dynamic and leakage energy reduction in time-constrained systems. In Proc. of ICCAD, 2004.

[2] L. Benini and G. De Micheli. Networks on chips: a new SoC paradigm. IEEE Computer, 35(1):70-78, 2002.

[3] D. Bertozzi, L. Benini, and G. De Micheli. Low power error resilient encoding for on-chip data buses. In Proc. of DATE, pages 102-109, 2002.

[4] W. Dally. Interconnect-limited VLSI architecture. In IEEE Conference on Interconnect Technologies, pages 15$17,1999$.

[5] J. Dielissen, A. Radulescu, K. Goossens, and E. Rijpkema. Concepts and implementation of the Philips networkon-chip. In IP-Based SoC Design, 2003.

[6] T. Dumitras and R. Marculescu. On-chip stochastic communication. In Proc. of DATE, 2003.

[7] F. Glover. Tabu search-Part I. ORSA J. Comput., 1989.

[8] J. Hu and R. Marculescu. DyAD-Smart routing for Network-on-Chip. In Proc. of DAC, 2004.

[9] D. Liu et al. Power consumption estimation in CMOS VLSI chips. IEEE J. of Solid-State Circuits, (29):663-670, 1994.

[10] S. Manolache. Fault-tolerant communication on network-on-chip. Technical report, Linkoping Univ., 2004.

[11] J. C. Palencia Gutiérrez and M. González Harbour. Schedulability analysis for tasks with static and dynamic offsets. In Proceedings of the 19th IEEE Real Time Systems Symposium, pages 26-37, December 1998.

[12] M. Pirretti, G. M. Link, R. R. Brooks, N. Vijaykrishnan, M. Kandemir, and I. M. J. Fault tolerant algorithms for network-on-chip interconnect. In Proc. of the ISVLSI, 2004. 


\title{
High Performance Computing on Fault-Prone Nanotechnologies: Novel Microarchitecture Techniques Exploiting Reliability-Delay Trade-offs
}

Andrey V. Zykov, Elias Mizan, Margarida F. Jacome, Gustavo de Veciana, Ajay Subramanian Department of Electrical and Computer Engineering, The University of Texas at Austin.

\begin{abstract}
Device and interconnect fabrics at the nanoscale will have a density of defects and susceptibility to transient faults far exceeding those of current silicon technologies. In this paper we introduce a new performance optimization dimension at the microarchitecture level which can mitigate overheads introduced by fault tolerance. This is achieved by directly exposing reliability versus delay design trade-offs while incorporating novel forms of speculation which use faster but less reliable versions of a microarchitecture's performance critical components. Based on a parameterized microarchitecture, we exhibit the benefits of optimizing these tradeoffs.

Keywords: Nanotechnologies, Fault Tolerant Microarchitectures, Performance Optimization, Reliability-Delay Trade-offs

\section{REFERENCES}

[1] G.Bourianoff, "The future of nanocomputing.” IEEE Computer Magazine, August 2003.

[2] Y. Huang et al., "Logic gates and computation from assembled nanowire building blocks," Science, vol. 294, 2001.

[3] D. Rotman, “The nanotube computer,” MIT Technology Review, March 2002.

[4] A. DeHon, “Array-based architecture for FET-based nanoscale electronics," IEEE Trans. on Computers, vol. 2, no. 1, March 2003.

[5] S. C. Goldstein and M. Budiu, "Nanofabrics: Spatial computing using molecular electronics," in Proc. ISCA 28, 2001.

[6] A.V. Zykov et al., "High performance computing on fault-prone nanotechnologies: Novel microarch. techn. exploiting reliability delay trade-offs," Tech. Rep., UT-CERC-TR-MJ-0502, 2005.

[7] T. Austin, "DIVA: A reliable substrate for deep submicron microarchitecture design," in Proc. Micro, November 1999.

[8] D. Burger and T. Austin, "The simplescalar tool set," Computer Architecture News, vol. 25, no. 3, June 1997.
\end{abstract}




\title{
How to Determine the Necessity for Emerging Solutions
}

Chair: $\quad$ Nic Mokhoff - EE Times, Manhasset, NY

Speakers: $\quad$ Kamalesh N. Ruparel - Cisco Systems, Inc., San Jose, CA

Hao Nham - eSilicon Corp., Bedminister, NJ

Francesco Pessolano - Philips Semiconductors, Eindhoven, Netherlands

Kee Sup Kim - Intel Corp., Sacramento, CA

\begin{abstract}
Different applications for today's chips require different type of optimizations and thus the need to adopt emerging products and solutions to meet such requirements. Optimizing for low power, for high yield, for reduced soft error or minimal bring up time necessitate adequate trade-off analysis and technical/business decision making by management. The lead managers in this session will discuss today's emerging solutions and their economic impact.
\end{abstract}


DAC05, pages 275-280

Closing the Power Gap between ASIC and Custom: An ASIC Perspective

D. G. Chinnery and K. Keutzer

Department of Electrical Engineering and Computer Sciences, University of California at Berkeley

\begin{abstract}
We investigate differences in power between application-specific integrated circuits (ASICs) and custom integrated circuits, with examples from 0.6 um to 0.13 um CMOS. A variety of factors cause synthesizable designs to consume $\times 3$ to $\times 7$ more power. We discuss the shortcomings of typical synthesis flows, and changes to tools and standard cell libraries needed to reduce power. Using these methods, we believe that the power gap between ASICs and custom circuits can be closed to within $\times 2$.
\end{abstract}

Keywords: ASIC, comparison, custom, energy, power, standard cell

\title{
REFERENCES
}

[1] ARM, ARM Processor Cores.

http://www.armdevzone.com/open.nsf/htmlall/A944EB65693A4EB180256A440051457A/\$File/ARM+cores+111-1.pdf

[2] A. Bhavnagarwala, et al., "A Minimum Total Power Methodology for Projecting Limits on CMOS GSI," IEEE Trans. VLSI Systems, vol. 8, no. 3, June 2000, pp. 235-251.

[3] T. Burd, et al., "A Dynamic Voltage Scaled Microprocessor System," in Proc. Int. Solid-State Circuits Conf., vol. 35, no. 11, 2000, pp. 1571-80.

[4] T. Callaway, and E. Swartzlander, "Optimizing Arithmetic Elements for Signal Processing," IEEE VLSI Signal Processing Workshop, 1992, pp. 91-100.

[5] D. Chinnery, and K. Keutzer, Closing the Gap Between ASIC \& Custom, Kluwer, 2002.

[6] L. Clark, et al., "An Embedded 32-b Microprocessor Core for Low-Power and High-Performance Applications," J. Solid-State Circuits, vol. 36, no. 11, Nov. 2001, pp. 1599-1608.

[7] M. Cote, and P. Hurat, "Faster and Lower Power Cell-Based Designs with Transistor-Level Cell Sizing," chapter 9 in Closing the Gap Between ASIC \& Custom, Kluwer, 2002.

[8] CPU Scorecard, Intel CPU Roster and AMD CPU Roster. http://www.cpuscorecard.com/cpuprices/

[9] L. Fanucci, and S. Saponara, "Data driven VLSI computation for low power DCT-based video coding," in Proc. Int. Conf. Electronics, Circuits and Systems, vol.2, 2002, pp. 541-4.

[10] S. Furber, ARM System-on-Chip Architecture. 2nd Ed. Addison-Wesley, 2000.

[11] J. Ganswijk, Chip Directory: ARM Processor family. http://www.xs4all.nl/ ganswijk/chipdir/fam/arm/

[12] J. Gong, et al., "Simultaneous buffer and wire sizing for performance and power optimization," in Proc. Int. Symp. on Low Power Electronics and Design, 1996, pp. 271-6.

[13] A. Harstein, and T. Puzak, "Optimum Power/Performance Pipeline Depth," in Proc. Int. Symp. on Microarchitecture, 2003, pp. 117-126.

[14] Intel, Intel XScale Microarchitecture: Benchmarks. http://developer.intel.com/design/intelxscale/ benchmarks.htm

[15] M. Levy, "Samsung Twists ARM Past 1GHz," Microprocessor Report, Oct. 16, 2002.

[16] J. Montanaro, et al., “A 160MHz, 32-b, 0.5W, CMOS RISC Microprocessor, ” J. Solid-State Circuits, vol. 31, no. 11, 1996, pp. 1703-14.

[17] B. Moyer, "Low-Power Design for Embedded Processors," Proc. IEEE, vol. 89, no. 11, Nov. $2001,1576-1587$.

[18] S. Narendra, et al., "Comparative Performance, Leakage Power and Switching Power of Circuits in $150 \mathrm{~nm}$ PDSOI and Bulk Technologies Including Impact of SOI History Effect," Int. Symp. on VLSI Circuits, 2001, pp. 217-8.

[19] S. Nassif, "Delay Variability: Sources, Impact and Trends," in Proc. Int. Solid-State Circuits Conf., 2000.

[20] D. Pradhan, et al., "Gate-Level Synthesis for Low-Power Using New Transformations," in Proc. Int. Symp. on Low Power Electronics and Design, 1996, pp. 297-300.

[21] R. Puri et al., "Pushing ASIC Performance in a Power Envelope," in Proc. Design Automation Conf., 2003, pp. 788-793.

[22] J. Quinn, Processor98: A Study of the MPU, CPU and DSP Markets, Micrologic Research, 1998.

[23] P. Simonen, et al., "Comparison of bulk and SOI CMOS Technologies in a DSP Processor Circuit Implementation," in Proc. Int. Conf. Microelectronics, 2001.

[24] D. Singh, et al., "Power Conscious CAD Tools and Methodologies: a Perspective," Proc. IEEE, vol. 83, no. 4, April 1995, pp. 570-94. 
[25] S. Sirichotiyakul, et al., "Stand-by Power Minimization through Simultaneous Threshold Voltage Selection and Circuit Sizing," in Proc. Design Automation Conf., 1999, pp. 436-41.

[26] D. Sylvester, and K. Keutzer, "Getting to the Bottom of Deep Submicron," in Proc. Int. Conf. on ComputerAided Design, 1998, pp. 203-11.

[27] Synopsys, Design Compiler User Guide, 2003.

[28] M. Takahashi, et al., "A 60-mW MPEG4 Video Codec Using Clustered Voltage Scaling with Variable SupplyVoltage Scheme," J. Solid-State Circuits, vol. 33, no. 11, 1998, pp. 1772-1780.

[29] K. Usami, and M. Igarishi, "Low-Power Design Methodology and Applications Utilizing Dual Supply Voltages," in Proc. ASP Design Automation Conf., 2000, pp. 123-8.

[30] H. Veendrick, "Short-circuit dissipation of static CMOS circuitry and its impact on the design of buffer circuits," J. Solid-State Circuits, vol. SC-19, August 1984, pp. 468-73.

[31] Virtual Silicon. http://www.virtual-silicon.com/

[32] T. Xanthopoulos, and A. Chandrakasan, "A Low-Power DCT Core Using Adaptive Bitwidth and Arithmetic Activity Exploiting Signal Correlations and Quantization," J. Solid-State Circuits, vol. 35, no. 5, May 2000, pp. 74050.

[33] T. Xanthopolous, and A. Chandrakasan, "A Low-Power IDCT Macrocell for MPEG-2 MP@ML Exploiting Data Distribution Properties for Minimal Activity,” J. Solid-State Circuits, vol. 34, May 1999, pp. 693-703. 


\title{
Explaining the Gap Between ASIC and Custom Power: A Custom Perspective
}

\author{
Andrew Chang ${ }^{1}$, William J. Dally ${ }^{2}$ \\ ${ }^{1}$ Cadence Design Systems, Inc., San Jose, CA \\ ${ }^{2}$ Stanford University, Gates CS Bldg. 3A-301, Stanford, University
}

\begin{abstract}
Power dissipation is now both a key constraint and an application driver in VLSI systems. For a specific application, the energy efficiency of different implementations can differ by multiple orders of magnitude. This work surveys a range of techniques available to improve energy efficiency and highlights their cumulative benefit. Understanding, adopting and adapting selected techniques from full-custom solutions can help bridge the efficiency gap for the ASIC designs. Architecture and microarchitecture choices yield multiple-order of magnitude improvements in power dissipation by matching the structure of the design to the structure of the application and by providing multiple operating and power-down modes. The combination of methodology and full-custom circuit techniques and libraries provide benefits primarily due to reduced parasitic loading enabling the improved performance to be translated into the potential for factor-of- 3 to factor-of-10 improvements in power.

Keywords: ASIC, Custom Circuits, EDA, Energy Efficiency, Low Power, Normalized Metrics, Technology Scaling
\end{abstract}

\section{REFERENCES}

[1] Agarwala, S., et al. A 600MHz VLIW DSP. IEEE Journal of Solid-State Circuits, 37, 11 (November, 2002), $1532-1544$.

[2] Baas, B. A Low-Power, High-Performance 1024-point FFT Processor. IEEE Journal of Solid-State Circuits, 34, 3 (March. 1999), 380-387.

[3] Chandrakasan, A., Bowhill, W., and Fox. F., Design of High-Performance Circuits. IEEE Press 2001.

[4] Chen, K., et al. Predicting CMOS Speed with Gate Oxide and Voltage Scaling and Interconnect Loading Effects. IEEE Transactions on Electron Devices, 44, 11 (November 1997), 1951-1957.

[5] Chinnery, D. G. and Keutzer, K., Closing the Gap Between ASIC and Custom. Kluwer Academic Press, Norwell, MA 2002.

[6] Dally, W. J. and Chang, A. The Role of Custom Design in ASIC Chips. In Proceedings of the 37th Design Automation Conference, Los Angeles, CA, June 5-9 2000. 643-647.

[7] Intel. StrongARM SA-1100 Microprocessor for Portable Applications Brief Datasheet. Intel, Chandler, AZ 1999.

[8] ITRS. International Technology Roadmap for Semiconductors 2001 Edition - System Drivers. ITRS. 2001.

[9] Magen, N. et al. Interconnect-Power Dissipation in a Microprocessor. In Proceedings of the 2004 International Workshop on System-Level Interconnect Prediction (Paris, France). 7-13.

[10] Lim, S.Y. and Crosland, A. Implementing FFT in an FPGA Co-Processor. In The International Embedded Solutions Event (GSPx). Santa Clara, CA, September 27-30, 2004.

[11] Rahal-Arabi, T. et al. Designing a 3GHz, 130nm, Intel Pentium 4. In Digest of Technical Papers, Symposium on VLSI Circuits (June 13-15, 2002), 130-133.

[12] Rixner, S. et al. A Bandwidth-Efficient Architecture for Media Processing. In Proceedings of the 31st Annual International Symposium on Microarchitecture (MICRO 31) (Dallas, TX). 3-13.

[13] Taur, Y., and Ning, T. Fundamentals of Modern VLSI Devices Cambridge University Press, Cambridge, CB2 1RP, United Kingdom 1998.

[14] Wang, A., and Chandrakasan, A. A 180-mV Subthreshold FFT Processor Using a Minimum Energy Design Methodology. IEEE Journal of Solid-State Circuits, 40, 1 (January. 2005), 310-319. 


\title{
Keeping Hot Chips Cool
}

\author{
Ruchir Puri*, Leon Stok**, Subhrajit Bhattacharya* \\ *IBM T J Watson Research Center, Yorktown Hts, NY \\ **IBM, Somers, NY
}

\begin{abstract}
With $90 \mathrm{~nm}$ CMOS in production and $65 \mathrm{~nm}$ testing in progress, power has been pushed to the forefront of design metrics. This paper will outline practical techniques that are used to reduce both leakage as well as active power in a standard-cell library based high-performance design flow. We will discuss the design and cost issues for using different power saving techniques such as: power gating to reduce leakage, multiple and hybrid threshold libraries for leakage reduction and multiple supply voltage based design. In addition techniques to reduce clock tree power will be presented as power consumed in clocks accounts for a significant portion of total chip power. Practical aspects of implementing these techniques will also be discussed.
\end{abstract}

Keywords: Low power, High-Performance, VLSI Design

\section{REFERENCES}

[1] Trevillyan, L. et al., An integrated design environment for technology closure of deep-submicron IC Designs, IEEE Design \& Test, Feb. 04.

[2] Puri, R. et al, Pushing ASIC performance in a power envelope, DAC 2003.

[3] Kosonocky, S. et al, Low Power Circuits and Technlogy for wireless digital systems, IBM Journal of R\&D, Vol. 47, No. 2/3, 2003.

[4] Puri, R., et al., Minimizing power with Flexible Voltage Islands, ISCAS 2005.

[5] Nassif, S., Delay Variability: Sources, Impact and Trends, ISSCC, 2000.

[6] Warnock, J., "Circuit Design with Leaky Transistor", ISSCC Workshop on Design in power constrainted era, 2003.

[7] Lackey, D. et al, "Managing power \& performance for SoC Designs using Voltage Islands", ICCAD 2002.

[8] Kim, S. et al., Understanding and minimizing ground bounce during mode transition of power gating structures., ISLPED 2003.

[9] Keshavarzi, A et al, Effectiveness of reverse body biasing for scaled technologies, ISLPED 2001.

[10] Roy, K., Clcok Gating for Microprocessor power reduction, High Performance Computer Architecture Symposium, 2003. 


\section{Interconnects Are Moving From MHz->GHz Should you be afraid?}

Or... "My Giga Hertz, Does Yours?"

Chair: $\quad$ Rick Merritt - EE Times, San Mateo, CA

Panelists: John F. D'Ambrosia - Tyco Electronics Corp., Harrisburg, PA

Adam Healey - Agere Systems, Inc., Allentown, PA

Boris Litinsky - RF Micro Devices, Inc., San Jose, CA

John Stonick - Synopsys, Inc., Hillsboro, OR

Joe Abler - IBM Corp., Research Triangle Park, NC

\section{PANEL ABSTRACT}

Chip interfaces, including standards like PCI Express, are increasingly relying on high-speed serial technology. This move from $\mathrm{MHz}$ to $\mathrm{GHz}$ brings with it a myriad of chip design challenges that many designers have never faced before. This diverse panel of experts in chip and IP mixed-signal design will describe not only why you SHOULD be afraid (very afraid), but also what's being done to make this transition practical, including new design techniques and standards. 


\title{
Design Methodology for Wireless Nodes with Printed Antennas
}

\author{
Jean-Samuel Chenard, Chun Yiu Chu, Željko Žilić, Milica Popović \\ Department of Electrical and Computer Engineering, McGill University, \\ Montréal, Québec, Canada
}

\begin{abstract}
The need for mass-produced inexpensive wireless devices operating under strict energy constraints poses new challenges in the system design methodology. This paper presents a methodology for designing wireless nodes in which a low cost, reliable antenna is realized by printed circuit traces. We show how to combine the analysis from 2.5D and 3D EM simulators with the PCB design tools to create predictable nodes with printed antennas that meet stringent power and data transmission range goals. The presented approach is applied to the design of a IEEE802.15.4 wireless node deployed in several indoor environments.
\end{abstract}

Keywords: RF CAD, Printed Antenna, Antenna Design Methodology, Printed Circuit Board

\section{REFERENCES}

[1] Agilent. Advanced design system 2003a documentation. Product documentation, Agilent Technologies, 2003.

[2] Chipcon. SmartRF CC2420 preliminary datasheet (rev 1.2). Datasheet, Chipcon AS, Gaustadall'een 21 NO0349, Oslo, NORWAY, 2004.

[3] H.-R. Chuang and L.-C. Kuo. 3-D FDTD design analysis of a 2.4-ghz polarization-diversity printed dipole antenna with integrated balun and polarization-switching circuit for WLAN and wireless communication applications. IEEE Trans. Microwave Theory Tech., 51(2), February 2003.

[4] D. Edward and D. Rees. A broadband printed dipole with integrated balun. Microwave J., pages 339-344, May 1987.

[5] M. Gimersky and J. Bornemann. A modified method-of-moments technique for the full-wave analysis of imperfect conductors on lossy and finite-extent substrates. IEEE MTT-S Int. Microwave Symp. Digest, 2:715-718, June 1996.

[6] W. H. Haydl. On the use of vias in conductor-backed coplanar circuits. IEEE Trans. Microwave Theory Tech., 50:1571-1577, June 2002.

[7] Y.-H. Pang and R.-B. Wu. Analysis of microstrip antennas with finite-sized substrate. IEEE AP-S Int. Symp., 2:814-817, July 2001.

[8] T. S. Rappaport. Wireless Communications: Principles and Practice. Prentice Hall, Upper Saddle River, New Jersey, $2^{\text {nd }}$ edition, 2002.

[9] M. Scott. A printed dipole for wide-scanning array application. 11th Int. Conf. Antennas and Propagation, 1:3740, 17-20 April 2001.

[10] C. Soras, M. Karaboikis, G. Tsachtsiris, and V. Mikios. Analysis and design of an inverted-F antenna printed on a PCMCIA card for the 2.4GHz ISM card. IEEE Antenna's and Propagation Magazine, 44:37-44, February 2002.

[11] W. L. Stutzman and G. A. Thiele. Antenna Theory and Design. Wiley, second edition, 1997.

[12] F. Xiao, K. Murano, and Y. Kami. The use of via holes for controlling the crosstalk of non-parallel microstrip lines on PCBs. 2002 IEEE Int. Symp. On Electromagnetic Compatibility, 2:633-638, August 2002.

[13] X. Ye, D. M. Hockanson, M. Li, Y. Ren, W. Cui, J. L. Drewniak, and R. E. DuBroff. EMI Mitigation with multilayer power-bus stacks and via stitching of reference planes. IEEE Trans. Electromagnetic Compatibility, 43:538-548, November 2001. 


\title{
MP Core: Algorithm and Design Techniques for Efficient Channel Estimation in Wireless Applications
}

Yan Meng, Andrew P. Brown, Ronald A. Iltis, Timothy Sherwood, Hua Lee, Ryan Kastner University of California, Santa Barbara, Santa Barbara, CA

\begin{abstract}
Channel estimation and multiuser detection are enabling technologies for future generations of wireless applications. However, sophisticated algorithms are required for accurate channel estimation and multiuser detection, and real-time implementation of these algorithms is difficult. This paper presents architectural design methods for wireless channel estimation which can be leveraged to enable real-time multiuser detection. We redesign the matching pursuit (MP) channel estimation algorithm to reduce the complexity while maintaining the estimation accuracy. Furthermore, we develop a parameterized intellectual property (IP) core, which provides a hardware implementation of the MP algorithm. Experimental results demonstrate the effectiveness and efficiency of the new algorithm and IP core for channel estimation. The implementation of our MP core on a modern, high performance reconfigurable system is about 216 times faster than running the algorithm on a state of the art microprocessor. The MP core possesses the speed required for performing true multiuser detection, enabling future generations of wireless communication applications.
\end{abstract}

Keywords: Channel estimation, matching pursuit algorithm, design space exploration

\section{REFERENCES}

[1] P. Belanovic and M. Leeser. A library of parameterized floating-point modules and their use. In FPL 2002, 2002.

[2] A. D. Blackowiak and S. D. Rajan. Multi-path estimates via optimizing highly oscillatory cost functions. In IEEE J. Oceanic Eng., volume 30, July 1998.

[3] S. F. Cotter and B. D. Rao. Sparse channel estimation via matching pursuit with application to equalization. IEEE Trans. Communications, 50:374-377, Mar. 2002.

[4] S. Mahlke et al. Bit-width cognizant architecture synthesis of custom hardware accelerators. In IEEE Trans. on Computer-Aided Design, Nov. 2001.

[5] J. J. Fuchs. Multipath time-delay detection and estimation. IEEE Trans. Signal Processing, 47:237-243, 1999.

[6] A. A. Gaffarl, O. Mencerl, W. Luk, and P. Y. K. Cheung. Unifying bit-width optimisation for fixed-point and floating-point designs. In IEEE Trans. on Computer-Aided Design, Nov. 2001.

[7] C. Huang, S. Ravi, A. Raghunathan, and N. K. Jha. High-level synthesis of distributed logic-memory architectures. In Proc. Int. Conf. Computer-Aided Design, Nov. 2002.

[8] R. A. Iltis and S. Kim. Geometric derivation of expectation-maximization and generalized successive interference cancellation algorithms with CDMA channel estimation. IEEE Trans. Signal Processing, 51(5):13671377, May 2003.

[9] M. F. Jacome and H. P. Peixoto. A survey of digital design reuse. In IEEE Design and Test of Computers, May 2001.

[10] S. Kim and R. A. Iltis. A matching pursuit/GSIC-based algorithm for DS-CDMA sparse channel estimation. IEEE Signal Processing Letters, 11:12-15, Jan. 2004.

[11] S. Meguerdichian, F. Koushanfer, A. Mogre, D. Petranovic, and M. Potkonjak. Metacores: design and optimization techinques. In $D A C$., Las Vegas, Nevada, June 2001.

[12] G. D Micheli. Synthesis and Optimization of Digital Circuits. McGraw-Hill, 1994.

[13] J. Proakis. Digital Communications. McGraw-Hill, New York, NY, 1995.

[14] S. Rajagopal, S. Bhashyam, and J. R. Cavallaro. Real-time algorithms and architectures for multiuser channel estimation and dectection in wireless base-station receivers. In IEEE Tran. on Wireless Comm., number 3, pages 374-377, July 2002.

[15] M. Stephenson, J.Babb, and S. Amarasinghe. Bitwidth analysis with application to silicon compilation. In IEEE Trans. on Computer-Aided Design, Nov. 2001.

[16] L. Vanzago, B. Bhattacharya, J. Cambonie, and L. Lavagno. Design space exploration for a wireless protocol on a reconfigurable platform. In DATE'03, Munich, Germany, Mar. 2003. 
[17] S. Verdú. Multiuser detection. Cambridge University Press, New York, Oct. 1998.

[18] Xilinx, INC. Virtex-II 1.5V Field Programmable Gated Arrays Datasheet, Oct. 2001. 


\title{
From Myth to Methodology: Cross-Layer Design for Energy-Efficient Wireless Communication
}

\author{
Wolfgang Eberle, Bruno Bougard, Sofie Pollin, Francky Catthoor \\ IMEC, and KU Leuven, Leuven, Belgium
}

\begin{abstract}
During the last decade, wireless communication has seen a trend towards application diversification leading to a significant growth in users. With the availability of - however energy-limited - nomadic devices and real-time multimedia applications, user demand is shifting from simply asking for higher data rates to more complex requirements in terms of Quality of Service (QoS) and energy-efficiency. In this new context energy management is becoming a key success factor. Optimized energy-efficiency requires an energy management that continuously trades off QoS and energy adapting to varying user expectations and environment dynamics. But, QoS can only be evaluated on top of the whole protocol stack while energy consumption largely appears at the lower layers. To minimize overhead during the transitions between layers, we need to address the problem from a cross-layer perspective. We present a methodology that, based on systematic exploration, effective problem partitioning and minimal cross-layer interface, allows energy management in a cross-layer way, while maintaining efficient layered semantics. Different case studies in the context of wireless LAN (WLAN) for multimedia and data traffic transport are discussed, to show how cross-layer energy management can easily be included in systems running state-of-the-art protocols.
\end{abstract}

Keywords: Cross-layer, Energy Management, Power-aware design

\section{REFERENCES}

[1] Intel Corp., "Low-power wireless LANs: Past, present, and Future,” panel session, DATE, Munich, March 2005.

[2] Dell TrueMobile 1400 WLAN Card.; available on http://support.ap.dell.com/docs/network/p44970/en/specs.html

[3] L. Benini et al., "A Survey of Design Techniques for System-Level Dynamic Power Mgmt," IEEE Trans. VLSI, vol. 8, no. 3, June 2000.

[4] T. Simunic et al., "Energy Efficient Design of Portable Wireless Systems," Proc. ISLPED, pp. 49-54, Italy, Aug. 2000 .

[5] R. Zheng and R. Kravets, "On-demand power management for ad hoc networks," Proc. IEEE INFOCOM, San Francisco, USA, 2003.

[6] IEEE 802.11 standard, specific parts on power management.

[7] A. Acquaviva, T. Simunic, et al., "Remote power control of wireless network interfaces," J. Embedded Comp., vol. 3, 2004.

[8] C. Schurgers et al., "Modulation scaling for energy aware communication systems," Proc. ISLPED, Huntington Beach, CA, Aug. 2001.

[9] E. Uysal-Biyikoglu, "Energy-Efficient Packet Transmission over a Wireless Link", ACM/IEEE Trans. Netw. vol. 10, no. 4, Aug. 2002.

[10] A. Sinha and A. P. Chandrakasan, "Energy Scalable System Design”, IEEE Trans.VLSI Systems, pp. 135-145, Apr. 2002.

[11] V. Kawadia, P. Kumar, "A cautionary perspective on cross-layer ," http://black.csl.uiuc.edu/ prkumar/ html_files/publications.html

[12] M Zargari et al., "A 5GHz CMOS Transceiver for IEEE 802.11a WLAN," IEEE JSSC, vol. 37, no. 12, pp. 1698-1694, Dec. 2002.

[13] A. Belnad et al., "Direct conv. CMOS transceiver with AFC for 802.11a WLANs," Proc. ISSCC, San Francisco, USA, Feb. 2003.

[14] B. Bougard et al., "Energy Scalability Enhancement of WLAN transceivers," IEEE SPAWC, Lisboa, Portugal, July 2004.

[15] Network simulator ns-2; available on http://www.isi.edu/nsnam/ns/.

[16] J. Van Driessche, G. Cantone, W. Eberle et al., "Transmitter cost/efficiency exploration for 5-GHz WLAN," IEEE Radio and Wireless Conf., Boston, USA, Aug. 2003. 
[17] K. M. Miettinen, Non-Linear Multi-Objective Optimization, Kluwer Academic Publisher, Boston, 1999.

[18] Microsemi, LX5503 WLAN Power Amplifier datasheet, http://www.microsemi.com/datasheets/lx5503.pdf.

[19] B. Bougard et al., "A scalable (...) parallel concatenated convolutional Turbo-Codec," Proc. ISSCC, San Francisco, USA, Feb. 2003.

[20] M. Tüchler et al., "Turbo equalization: principles and new results," IEEE Trans. Comm., vol. 50, pp. 754-767, May 2002.

[21] B. Bougard et al., "Energy-aware radio link control for OFDMbased WLAN", Proc. IEEE SiPS, Austin, USA, Oct. 2004.

[22] R. Berry and R. Gallager, "Communication over fading channels with delay constraints," IEEE Trans. Inform. Theory, vol. 48, pp. 1135-1149, May 2002.

[23] B. Bougard et al., "Transport level performance-energy trade-off in wireless networks and consequences on the system-level architecture and design paradigm", Proc. IEEE SiPS, Austin, USA, Oct. 2004.

[24] R. Jain, "Quality of Experience," IEEE Multimedia, vol. 11, no. 1 pp. 95-96, Jan-Mar 2004.

[25] A. van Moorsel, "Metrics for the internet age: Quality-of-Experience and Quality of Business," HP Labs report HPL-2001-179, 2001.

[26] R. Mangharam, S. Pollin, et al., "Optimal fixed and scalable energy mgmt for wireless networks," IEEE INFOCOM, Miami, Mar 2005.

[27] J.-R. Ohm, “Advances in scalable video coding," Proc. IEEE, vol. 93, no. 1, pp. 42-56, Jan. 2005. 


\title{
An Efficient Algorithm for Statistical Minimization of Total Power under Timing Yield Constraints
}

\author{
Murari Mani ${ }^{1}$, Anirudh Devgan ${ }^{2}$, and Michael Orshansky ${ }^{1}$ \\ ${ }^{1}$ University of Texas, Austin \\ ${ }^{2}$ Magma Design Automation
}

\begin{abstract}
Power minimization under variability is formulated as a rigorous statistical robust optimization program with a guarantee of power and timing yields. Both power and timing metrics are treated probabilistically. Power reduction is performed by simultaneous sizing and dual threshold voltage assignment. An extremely fast run-time is achieved by casting the problem as a secondorder conic problem and solving it using efficient interior-point optimization methods. When compared to the deterministic optimization, the new algorithm, on average, reduces static power by $31 \%$ and total power by $17 \%$ without the loss of parametric yield. The run time on a variety of public and industrial benchmarks is $30 \mathrm{X}$ faster than other known statistical power minimization algorithms.
\end{abstract}

Keywords: Leakage, manufacturability, statistical optimization

\section{REFERENCES}

[1] C. Visweswariah, "Death, taxes and failing chips," Proc. of DAC 2003, pp. 343-347.

[2] Y. Taur et al., "CMOS scaling into the nanometer regime," Proc. of the IEEE, no. 4, 1997, pp. 486-504.

[3] R. Brodersen et al., "Methods for True Power Minimization," in Proc. Of ICCAD, 2002, pp. 35-40.

[4] S. Borkar et al., "Parameter variation and impact on Circuits and Microarchitecture," Proc. of DAC, 2003, pp. 338-342.

[5] S. Sirichotiyakul et al., "Stand-by power minimization through simultaneous threshold voltage selection and circuit sizing," Proc. of DAC, 1999, pp. 436-441.

[6] Q. Wang, and S. Vrudhula, "Static power optimization of deep submicron CMOS circuit for dual Vth technology," Proc. of ICCAD, 1998, pp. 490-496.

[7] D. Nguyen et al., "Minimization of dynamic and static power through joint assignment of threshold voltages and sizing optimization," Proc. of ISLPED, 2003, pp. 158 - 163.

[8] H. Chang and S. Sapatnekar, "Statistical timing analysis considering spatial correlations using a single PERTlike traversal," Proc. of ICCAD, 2003, pp. $621-625$.

[9] X. Bai et al., "Uncertainty aware circuit optimization," Proc. of DAC, 2002, pp. 58 - 63.

[10] S. Raj et al., "A methodology to Improve Timing Yield," Proc. of DAC, 2004, pp. 448-453.

[11] A. Srivastava, D. Sylvester, and D. Blaauw, "Statistical optimization of leakage power considering process variations using dual-Vth and sizing," Proc. of DAC, June 7-11, 2004 pp. $773-778$.

[12] E. Jacobs and M. Berkelaar, "Gate sizing using a statistical delay model," Proc. of DAC, 2000, pp. 283-290.

[13] P. Seung et al., "Novel sizing algorithm for yield improvement under process variation in nanometer technology", Proc. of DAC, 2004, June 7-11, 2004, pp. $454-459$.

[14] M. Mani and M. Orshansky, “A new statistical optimization algorithm for gate sizing," Proc. of ICCD, 2004, pp. $272-277$.

[15] J. Fishburn and A. Dunlop, "TILOS: A Posynomial Programming Approach to Transistor Sizing," Proc. of ICCAD, 1985, pp. 326-328.

[16] D. Markovic et al., "Methods for true energy-performance optimization," J. of Solid-State Circuits, 2004, pp. 1282- 1293.

[17] V. Sundararajan et al., "Fast and Exact Transistor sizing Based on Iterative Relaxation," IEEE Trans. on CAD, vol. 21, 2002, pp.568-581.

[18] J. Kao et al., "Subthreshold Leakage Modeling and Reduction Techniques," Proc. of ICCAD, 2002, pp. 141149.

[19] C. Chatfield, Introduction to Multivariate analysis, Chapman and Hall, 1980.

[20] S. Boyd, L. Vandenberghe, Convex Optimization, Cambridge, 2004.

[21] Kim et al., "A Heuristic for Optimizing Stochastic Activity Networks with Applications to Statistical Circuit Sizing", preprint. 
[22] A. Prekopa, Stochastic Programming, Kluwer Academic, 1995

[23] http://www.mosek.com/documentation.html\#manuals

[24] Y. Cao et al., "New paradigm of predictive MOSFET and interconnect modeling for early circuit design," Proc. of IEEE CICC, 2000, pp. 201-204.

[25] M. Pelgrom et al., Matching Properties of MOS Transistors, IEEE Journal of Solid-State Circuits, vol. 24, 1989, pp. 1433-1440. 


\title{
Robust Gate Sizing by Geometric Programming
}

Jaskirat Singh, Vidyasagar Nookala, Zhi-Quan Luo, Sachin Sapatnekar

Department of Electrical and Computer Engineering, University of Minnesota, Minneapolis, MN

\begin{abstract}
We present an efficient optimization scheme for gate sizing in the presence of process variations. Using a posynomial delay model, the delay constraints are modified to incorporate uncertainty in the transistor widths and effective channel lengths due to the process variations. An uncertainty ellipsoid method is used to model the random parameter variations. Spatial correlations of intradie width and channel length variations are incorporated in the optimization procedure. The resulting optimization problem is relaxed to be a Geometric Program and is efficiently solved using convex optimization tools. The effectiveness of our robust gate sizing scheme is demonstrated by applying the optimization on the ISCAS ' 85 benchmark circuits and testing the optimized circuits by performing Monte Carlo simulations to model the process variations. By varying the size of the uncertainty ellipsoids, a trade-off between area and robustness is explored. Experimental results show that the timing yield of the robustly optimized circuits improves manifold over the traditional deterministically sized circuits. As compared to the worst-case design, the robust gate sizing solution having the same area, has fewer timing violations.
\end{abstract}

Keywords: Geometric Program, posynomial, uncertainty ellipsoid

\section{REFERENCES}

[1] J. Fishburn and A. Dunlop, “TILOS: A Posynomial Programming Approach to Transistor Sizing," in Proc. IEEE/ACM ICCAD, pp. 326-328, 1985.

[2] S. Sapatnekar, V. B. Rao, P. M. Vaidya, and S. M. Kang, "An Exact Solution to the Transistor Sizing Problem for CMOS Circuits Using Convex Optimization," in IEEE Trans. on CAD, vol. 12, pp. 1621-1634, Nov 1993.

[3] K. Kasamsetty, M. Ketkar, and S. S. Sapatnekar, "A New Class of Convex Functions for Delay Modeling and their Application to the Transistor Sizing Problem," in IEEE Trans. on CAD, vol. 19, pp. 779-788, Jul 1998.

[4] X. Bai, C. Visweswariah, P. N. Strenski, and D. J. Hathaway, "Uncertainty-Aware Circuit Optimization," in Proc. ACM/IEEE DAC, pp. 58-63, 2002.

[5] S. Raj, S. B. K. Vrudhala, and J. Wang, "A Methodology to Improve Timing Yield in the Presence of Process Variations," in Proc. ACM/IEEE DAC, pp. 448-453, 2004.

[6] S. H. Choi, B. C. Paul, and K. Roy, "Novel Sizing Algorithm for Yield Improvement under Process Variation in Nanometer Technology," in Proc. ACM/IEEE DAC, pp. 454-459, 2004.

[7] E. T. A. F. Jacobs and M. R. C. M. Berkelaar, "Gate Sizing Using a Statistical Delay Model," in Proc. IEEE DATE, pp. 283-291, 2000.

[8] S. Boyd and L. Vandenberghe, Convex Optimization. Cambridge University Press, Cambridge, UK, 2004.

[9] R. A. Johnson and D. W. Wichern, Applied Multivariate Statistical Analysis. Prentice Hall, Upper Saddle River, NJ, 2002.

[10] K. L. Hsiung, S. J. Kim and S. Boyd, "Robust Geometric Programming via Piecewise Linear Approximation," 2003. Submitted to Mathematical Programming, available at http://www.stanford.edu//boyd/rgp.html.

[11] H. L. A-Malek and A.-K. S. O. Hassan, "The Ellipsoidal Technique for Design centering and Region Approximation," in IEEE Trans. on CAD, vol. 10, pp. 1006-1014, Aug 1991.

[12] S. Sapatnekar, P. M. Vaidya, and S. M. Kang, "Convexity-based Algorithms for Design Centering," in IEEE Trans. on CAD, vol. 13, pp. 1536-1549, Dec 1994.

[13] H. Chang and S. S. Sapatnekar, "Statistical Tming Analysis Considering Spatial Correlations Using a Single PERT-like Traversal," in Proc. IEEE/ACM ICCAD, pp. 621-625, 2003.

[14] A. Agarwal, D. Blaauw, and V. Zoltov, "Statistical Timing Analysis for Intra-Die Process Variations with Spatial Correlations," in Proc. IEEE/ACM ICCAD, pp. 900-907, 2003.

[15] S. Nassif, "Delay Variability: Sources, Impact and Trends," in Proc. ISSCC, pp. 368-369, 2000.

[16] Available at http://www.mosek.com.

[17] "TSMC: 180nm Test Data." Available at http://www.mosis.org/Technical/Testdata/tsmc-018-prm.html.

[18] A. Caldwell, A. B. Kahng and I. Markov, "Capo: a largescale fixed-die placer." available at http://vlsicad. ucsd. edu/GSRC/bookshelf/Slots/Placement. 


\title{
Circuit Optimization using Statistical Static Timing Analysis
}

\author{
Aseem Agarwal*, Kaviraj Chopra*, David Blaauw*, Vladimir Zolotov** \\ *University of Michigan, Ann Arbor, MI \\ **IBM T.J.Watson, Yorktown heights, NY
}

\begin{abstract}
In this paper, we propose a new sensitivity based, statistical gate sizing method. Since circuit optimization effects the entire shape of the circuit delay distribution, it is difficult to capture the quality of a distribution with a single metric. Hence, we first introduce a new objective function that provides an effective measure for the quality of a delay distribution for both ASIC and high performance designs. We then propose an efficient and exact sensitivity based pruning algorithm based on a newly proposed theory of perturbation bounds. A heuristic approach for sensitivity computation which relies on efficient computation of statistical slack is then introduced. Finally, we show how the pruning and statistical slack based approaches can be combined to obtain nearly identical results compared with the brute-force approach but with an average run-time improvement of up to $89 \mathrm{x}$. We also compare the optimization results against that of a deterministic optimizer and show an improvement up to $16 \%$ in the 99-percentile circuit delay and up to $31 \%$ in the standard deviation for the same circuit area.
\end{abstract}

General Terms: Algorithms, performance, reliability, optimization

\section{References}

[1] S. Nassif, "Delay Variability: Sources, Impacts and Trends," Proceedings of ISSCC, 2000.

[2] A. Agarwal, D. Blaauw, V. Zolotov, S. Vrudhula, "Computation and Refinement of Statistical Bounds on Circuit Delay," DAC 2003.

[3] A. Devgan, C. Kashyap, "Block-based Static Timing Analysis with Uncertainty," ICCAD 2003, pp.607-614.

[4] H. Chang, S. Sapatnekar, "Statistical Timing Analysis Considering Spatial Correlations using a Single Pert-like Traversal," ICCAD'03.

[5] H. Hashimoto, H. Onodera. "Increase in delay uncertainty by performance optimization", ISCAS 2001, pp. 379382.

[6] X. Bai, C. Visweswariah, P. N. Strenski, and D. J. Hathaway. "Uncertainty-aware circuit optimization”, DAC 2002, pp. 58-63

[7] E.T.A.F. Jacobs, M.R.C.M Berkelaar. "Gate sizing using a statistical delay model”, DATE 2000, pp. 283-289.

[8] S. Raj, S. Vrudhula, J. Wang. "A methodology to improve timing yield in the presence of process variations", DAC 2004.

[9] A. Agarwal, K. Chopra, D. Blaauw, "Statistical Timing Based Optimization using Gate Sizing”, DATE 2005.

[10] K. Bowman et. al., "Impact of die-to-die and within-die Parameter Fluctuations on the Maximum Clock Frequency Distribution for Gigascale Integration,” IEEE J. Solid-State Circuit, Feb 2002.

[11] Personal communication, Kerry Bernstein, IBM Corp, Burlington, VT.

[12] F. Brglez, H.Fujiwara, "A Neutral Netlist of 10 Combinatorial Benchmark Circuits", Proc. ISCAS, 1985, pp.695-698. 


\title{
An Exact Jumper Insertion Algorithm for Antenna Effect Avoidance/Fixing
}

\section{Bor-Yiing Su*, Yao-Wen Chang**}

*Department of Electrical Engineering, National Taiwan University, Taipei, Taiwan

**Graduate Institute of Electronics Engineering and Department of Electrical Engineering,

National Taiwan University, Taipei, Taiwan

\begin{abstract}
As the process technology enters the nanometer era, reliability has become a major concern in the design and manufacturing of VLSI circuits. In this paper we focus on one reliability issuejumper insertion in routing trees for avoiding/fixing antenna effect violations at the routing/postlayout stages. We formulate the jumper insertion for antenna avoidance/fixing as a tree-cutting problem. We show that the tree-cutting problem exhibits the properties of optimal substructures and greedy choices. With these properties, we present an $O(V \lg V)$-time exact jumper insertion algorithm that uses the optimum number of jumpers to avoid/fix the antenna violations in a routing tree with $V$ vertices. Experimental results show the superior effectiveness and efficiency of our algorithm.
\end{abstract}

Keywords: Antenna Effect, Jumper Insertion

\section{REFERENCES}

[1] P. H. Chen, S. Malkani, C.-M. Peng, and J. Lin, "Fixing antenna problem by dynamic diode dropping and jumper insertion", Proc. ISQED, pp 275-282, 2000.

[2] T. H. Cormen, C. E. Leiserson, R. L. Rivest, and C. Stein, Introduction to Algorithms, McGraw-Hill Book Co., $2^{\text {nd }}$ Ed., 2001.

[3] T.-Y. Ho, Y.-W. Chang, and S.-J. Chen, "Multilevel routing with antenna avoidance," Proc. ISPD, April 2004.

[4] H. Shin, C. -C. King, and C. Hu, "Thin Oxide Damage by plasma etching and ashing process", Proc. IRPS, 1992.

[5] H. Watanabe, et al., "A wafer level monitoring method for plasma-charging damage using antenna PMOSFET test structure," IEEE Trans. Semiconductor manufacturing, vol. 10, no. 2, May. 1997. 


\title{
Fine-grained Application Source Code Profiling for ASIP Design
}

\author{
Kingshuk Karuri, Mohammad Abdullah Al Faruque, Stefan Kraemer, Rainer Leupers, \\ Gerd Ascheid, Heinrich Meyr \\ Institute for Integrated Signal Processing Systems, RWTH Aachen, Germany
}

\begin{abstract}
Current Application Specific Instruction set Processor (ASIP) design methodologies are mostly based on iterative architecture exploration that uses Architecture Description Languages (ADLs) and retargetable software development tools. However, for improved design efficiency, additional pre-architecture exploration tools are required to help narrow-down the huge design space and making coarsegrained Instruction Set Architecture (ISA) decisions before detailed ADL modeling. Extensive application code profiling is the key in such early design stages. Based on a novel code instrumentation technology, we present a microprofiling approach that fills the current gap between sourcelevel and instruction-level profilers and combines their advantages w.r.t. speed and accuracy. We show how the microprofiler is embedded into an advanced ASIP design flow and justify its use in a case study to design an MP3 decoder ASIP.
\end{abstract}

Keywords: Customizable Processors, ASIPs, Profiling, Codesign

\section{REFERENCES}

[1] J.A. Fisher: Customized Instruction Sets for Embedded Processors, Design Automation Conference (DAC), 1999 [2] A. Oraioglu, A. Veidenbaum: Application Specific Microprocessors (Guest Editors' Introduction), IEEE Design \& Test Magazine, Jan/Feb 2003

[3] F. Sun, S. Ravi, A. Ragnunathan, N.K. Jha: Synthesis of Custom Processors based on Extensible Platforms, ICCAD, 2002

[4] D. Goodwin, D. Petkov: Automatic Generation of Application Specific Processors, CASES, 2003

[5] H. Scharwaechter, D. Kammler, A. Wieferink et al.: ASIP Archiecture Exploration for Efficient IPsec Encryption, SCOPES, 2004

[6] K. Atasu, L. Pozzi, P. Ienne: Automatic Application-Specific Instruction-Set Extensions under Microarchitectural Constraints, DAC, 2003

[7] N. Clark, H. Zhong, S.Mahlke: Processor Acceleration Through Automated Instruction Set Customization, MICRO-36, 2003

[8] SpixTools: Introduction and User's Manual, TR 93-6, Sun Microsystems, Feb 1993

[9] VTune: http://www.intel.com/software/products/vtune

[10] D. Suresh, W. Najjar, F. Vahid et al.: Profiling Tools for Hardware/Software Partitioning of Embedded Applications, LCTES, 2003

[11] LISATek products: http://www.coware.com

[12] A. Nohl, G. Braun, A. Hoffmann et al.: A Universal Technique for Fast and Flexible Instruction Set Architecture Simulation, DAC, 2002

[13] M. Reshadi, P. Mishra, N. Dutt: Instruction Set Compiled Simulation: A Technique for Fast and Flexible Instruction Set Simulation, DAC, 2003

[14] M. Ravasi, M. Mattavelli: High-level Algorithmic Complexity Evaluation for System Design, Journal of Systems Architecture, no. 48, Elsevier, 2003

[15] L. Cai, A. Gerstlauer, D. Gajski: Retargetable Profiling for Rapid, Early System-level Design Space Exploration, DAC, 2004

[16] A. Halambi, P. Grun, V. Ganesh, A. Khare, N. Dutt A. Nicolau: EXPRESSION: A Language for Architecture Exploration through Compiler/Simulator Retargetability, DATE, 1999

[17] R. Leupers, O. Wahlen, M. Hohenauer et al.: An Executable Intermediate Representation for Retargetable Compilation and High-Level Code Optimization, Int. Workshop on Systems, Architectures, Modeling, and Simulation (SAMOS), 2003

[18] R. A. Uhlig, T. N. Mudge : Trace-driven Memory Simulation : A Survey, Proceedings of the ACM Computing Surveys, 1997

[19] Dinero : http://www.cs.wisc.edu/markhill/dineroiv/

[20] MPG123 Distribution : http://ftp.tu-clausthal.de/pub/unix/audio/mpg123 
[21] Softfloat distribution : http://www.cs.berkeley.edu/ jhauser/arithmetic/SoftFloat.html

[22] P. Giusto, G. Martin, E. Harcourt : Reliable Estimation of Execution Time of Embedded Software, DATE, 2001

[23] L. Lavagno, J. R. Bammi, E. Harcourt et al: Software Performance Estimation Strategies in a System-level Design Tool, CODES, 2000 
DAC05, pages 335-340

\title{
Physically-Aware HW-SW Partitioning for Reconfigurable Architectures with Partial Dynamic Reconfiguration
}

\author{
Sudarshan Banerjee, Elaheh Bozorgzadeh, Nikil Dutt \\ Center for Embedded Computer Systems, University of California, Irvine, CA, USA
}

\begin{abstract}
Many reconfigurable architectures offer partial dynamic configurability, but current system-level tools cannot guarantee feasible implementations when exploiting this feature. We present a physically aware hardware-software (HW-SW) scheme for minimizing application execution time under HW resource constraints, where the HW is a reconfigurable architecture with partial dynamic reconfiguration capability. Such architectures impose strict placement constraints that lead to implementation infeasibility of even optimal scheduling formulations that ignore the nature of these constraints. We propose an exact and a heuristic formulation that simultaneously partition, schedule, and do linear placement of tasks on such architectures. With our exact formulation, we prove the critical nature of placement constraints. We demonstrate that our heuristic generates high-quality schedules by comparing the results with the exact formulation for small tests and a popular, but placementuanaware scheduling heuristic for larger tests. With a case study, we demonstrate extension of our approach to handle heterogenous architectures with specialized resources distributed between general purpose programmable logic columns. The execution time of our heuristic is very reasonable- task graphs with hundreds of nodes are processed in a couple of minutes.
\end{abstract}

Keywords: HW-SW partitioning, partial dynamic reconfiguration, linear placement

\section{REFERENCES}

[1] P-H Yuh, C-L Yang, Y-W Chang, H-L Chen, "Temporal floorplanning using the T-tree formulation”, ICCAD, 2004

[2] S. Ghiasi, M. Sarrafzadeh, "Optimal Reconfiguration Sequence Management”, ASPDAC, 2003.

[3] J. L. Ramirez-Alfonsin, B. A. Reed (Eds.), "Perfect Graphs”, John Wiley and Sons, 2001.

[4] S.P. Fekete, E.Kohler, J.Teich, "Optimal FPGA module placement with temporal precedence constraints", DATE, 2001

[5] H. Singh, G. Lu, E. M. C. Filho, R. Maestre, M-H. Lee, F. J. Kurdahi, N. Bagherzadeh, ”MorphoSys: case study of a reconfigurable computing system targeting multimedia applications", DAC, 2000.

[6] B. Mei, P. Schaumont, S. Vernalde, "A hardware-Software Partitioning and scheduling algorithm for dynamically reconfigurable embedded systems", ProRisc workshop on Ckts, Systems and Signal processing, Nov 2000.

[7] B. Jeong, S. Yoo, S. Lee, K. Choi, "Hardware-Software Cosynthesis for Run-time Incrementally Reconfigurable FPGAs", ASPDAC, 2000.

[8] K. S. Chatha, R. Vemuri, "An iterative algorithm for Hardware-Software partitioning, Hardware design Space Exploration, and scheduling", Jrnl Design Automation for Embedded Systems, V-5, 2000

[9] S. Hauck, "Configuration pre-fetch for single context reconfigurable processors", FPGA, 1998.

[10] R P Dick, D L Rhodes, W Wolf, "TGFF: task graphs for free”, CODES 1998

[11] F. Vahid, T. D. Le, "Extending the Kernighan-Lin heuristic for Hardware and Software functional partitioning", Jrnl Design Automation for Embedded Systems, V-2, 1997

[12] M. J.Wirthlin, "Improving functional density through Run-time Circuit Reconfiguration", PhD Thesis, Electrical and Computer Engineering Dept, Brigham Young Univesity, 1997.

[13] H. Murata, K. Fujiyoshi, S. Nakatake, Y. Kajitani, "Rectangle-packing based module placement", ICCAD, 1995

[14] C. M. Fiduccia, R. M. Mattheyes, "A Linear-time heuristic for improving network partitions", DAC, 1982

[15] B Kernighan, S Lin, "An efficient heuristic procedure for partitioning graphs", The Bell System Technical Journal, V-29, 1970

[16] S Banerjee, E Bozorgzadeh, N Dutt, "HW-SW partitioning for architectures with partial dynamic reconfiguration", Technical Report CECS-TR-05-02, UC Irvine. 
[17] www.xilinx.com 


\title{
Performance Simulation Modeling for Fast Evaluation of Pipelined Scalar Processor by Evaluation Reuse
}

\author{
Ho Young Kim and Tag Gon Kim \\ Systems Modeling Simulation Laboratory, Dept of EECS, KAIST, Korea
}

\begin{abstract}
This paper proposes a rapid and accurate evaluation scheme for cycle counts of a pipelined processor using evaluation reuse technique. Since exploration of an optimal processor is a timeconsuming task due to large design space, fast evaluation methodology for an architecture is crucial. We introduce the performance simulation model which can evaluate the performance without considering the functional correctness. This model has an FSM-like form and can afford to take all hazard types of pipelined architectures into consideration. The proposed approach is based on the property that an application program, especially multimedia application, has many iterative loops in general. This property invokes many iterative operations in the simulation. Evaluation reuse scheme can alleviate redundantly iterative operations of conventional simulators in the loop. A performance simulator for the pipeline architecture has been developed through which greater speedup has been made compared with other approaches in the evaluation of cycle counts.
\end{abstract}

Keywords: Retargetable simulation, compiled simulation, evaluation reuse, instruction set architecture, trace-driven simulation

\section{REFERENCE}

[1] A. Hoffmann, A.Nohl, G.Braun, O.Schliebusch, T.Kogel and H.Meyr, "A Novel Methodology for the Design of Application Specific Instruction Set Processors using a Machine Description Language", IEEE Trans. Computeraided Design of Integrated Circuits and Systems, vol. 20, no. 11, pp. 1338-1354, November 2001.

[2] G. Lauterbach, "Accelerating Architectural Simulation by Parallel Execution of trace samples", in Proc. of 27th Hawaii International Conference on System Science, vol. 1, pp.205-210, January, 1994

[3] J. Liu, M.Lajolo and A. Sangiovanni-Vincentelli, "Software timing analysis using HW/SW cosimulation and instruction set simulator," in Porc. Int. Workshop. Hardware-Software Codesign, Mar. 1998, pp.65-70

[4] A. Nohl et al. "A Universal Technique for Fast and Flexible Instruction-Set Architecture Simulation", Design Automation Conference, pp. 22-27, June, 2002

[5] I. Park, S. Kang and Y Yi, "Fast cycle-accurate Behavioral Simulation for Pipelined Processors Using Early Pipeline Evaluation," International Conference on Computer Aided Design, pp. 138-141, Nov, 2003

[6] Jianwen Zhu et al. "An Ultra-Fast Instruction Set Simulator," Transaction on VLSI Systems, vol. 10, no. 3 pp.363-373, June 2002

[7] J. K. Kim and T. G. Kim, "Trace-driven Rapid Pipeline Architecture Evaluation Scheme for ASIP Design", in Proc. of Asia South-Pacific Design Automation Conference, Kitakyushu, Japan, pp.129-134, January, 2003

[8] J. L. Hennessey and D. A. Patterson, Computer Architecture: a quantitative approach, Morgan Kaufmann Publisher, 1990

[9] J. K. Kim, H. Y. Kim and T. G. Kim, "Top-down Retargetable Framework with Token-level Design for Accelerating Simulation Time of Processor Architecture", IEICE Trans. Fundamentals of Electronics, Communications and Computer Sciences, vol. E86-A, no. 12, pp.3089-3098, December 2003

[10] Mark D. Hill, "DINERO IV Trace-Driven Uniprocessor Cache Simulator", www.cs.wisc.edu/ markhill/ DineroIV 


\title{
Trace-Driven HW/SW Cosimulation Using Virtual Synchronization Technique
}

\author{
Dohyung Kim, Youngmin Yi, Soonhoi Ha
}

School of Computer Science and Engineering, Seoul Nation University, Seoul, Korea

\begin{abstract}
Poor performance of $\mathrm{HW} / \mathrm{SW}$ cosimulation is mainly caused by synchronization requirement between component simulators. Virtual synchronization technique was proposed to remove the need of synchronization in cycle accurate cosimulation. But the previous execution-driven simulation based on virtual synchronization has limitations in the application area. In this paper, we propose a novel trace-driven HW/SW cosimulation using virtual synchronization technique. Through OS modeling and channel modeling, the proposed cosimulation technique could be applied more widely while improving the simulation performance further. Experiments with a DIVX player example prove the viability of the proposed technique.
\end{abstract}

Keywords: Trace-driven cosimulation, virtual synchronization

\section{REFERENCES}

[1] D. Kim, C. Rhee, and S. Ha, "Combined Data-driven and Event-driven Scheduling Technique for Fast Distributed Cosimulation", IEEE Transactions on VLSI Systems Vol. 10 pp 672-679 Oct. 2002

[2] Y. Yi, D. Kim, S. Ha, "Fast and Time-Accurate Cosimulation with OS Scheduler Modeling", Design Automation for Embedded Systems, Kluwer Academic Publishers Vol. 8 pp 211-228 Sep. 2003

[3] B. Bailey, "Co-Verification: From Tool to Methodology," Mentor Consulting Technical Publication, June 2002

[4] T. Grotker, S. Liao, G. Martin and S. Swan, "System Design with SystemC", Kluwer Academic, Norwell, Mass., 2002

[5] K. Hines and G. Borriello, "Optimizing communication in embedded system cosimulation”, in Proc. Intl. Symp. on Hardware/Software Codesign, pp.121-125, Mar. 1997.

[6] Wonyong Sung and Soonhoi Ha, "Optimized Timed Hardware Software Cosimulation without Roll-back", DATE 98, Paris, France February 1998

[7] S. Yoo and K. Choi, "Optimistic Distributed Timed Cosimulation Based on Thread Simulation Model”, Proc. Of Proc. 6th Int’l Workshop on HW/SW Co-Design, Mar. 1998 


\title{
The Titanic: What Went Wrong!
}

Organizer: Sani R. Nassif-IBM Research, Austin, TX

\begin{abstract}
We often hear about success stories in EDA. We are all justifiably proud of the impact we collectively make on the overall integrated circuit design and manufacturing machine. It is fair to say, however, the one learns far more from failure than one does from success. In this special session we found several brave practitioners who are willing to talk about problems in businessas-usual EDA. These problems include technology related issues; reliability related issues, power issues and even methodology issues - In short, covering a wide swatch of the EDA domain.
\end{abstract}

Metal Variation-Induced Hold Time Failures

Author: Paul S. Zuchowski - IBM, Burlington, VT

Design for Reliability: acknowledging aging effects

Authors: Claude Moughanni, Mohamed Moosa, Gary Anderson - Freescale, Austin, TX

Bridging the Power Reduction \& Estimation Gap in the Cell Processor Design Methodology

Author: Stephen D Posluszny - IBM, Austin, TX

A Series of Unfortunate Events

Author: Ward Vercruysse - AMD, Austin, TX 


\section{Wireless Platforms: GOPS for Cents and MilliWatts}

Chair: Jan Rabaey - UC Berkeley, Berkeley, CA

Panelists: $\quad$ Allan Cox - 3Plus1 Technology, Inc., Saratoga, CA

Frank Lane - Flarion Technolgies, Inc., Bedminster, NJ

Rudi Lauwereins - IMEC, Belgium

Ulrich Ramacher - Infineon Technologies AG, Muenchen, Germany

David Witt - Texas Instruments, Dallas, TX

\section{PANEL SUMMARY}

In recent years, data communication has overtaken voice as the main force behind the growth in wireless. With this has come a proliferation of standards ranging from wide area networks at one end of the spectrum to personal area networks on the other end. The opportunities offered by this truly ubiquitous connectivity are tremendous, and are leading to revolutionary chances in the way computer, communication, and consumer systems operate and interact.

Providing the necessary flexibility to seamlessly interact with the multitude of emerging network models, as well as the muscle to support the demanding multimedia functionality in a mobile environment, presents some huge challenges to the developer of the wireless implementation platforms. The power budget of the mobile terminal is typically fixed by size considerations and operation time. Cost considerations further constrain the solution space.

In response to these challenges, many solutions have been floated and experimented with ranging from multi-processor architectures, advanced DSPs, reconfigurable solutions and hardwired accelerators. While these innovations break new ground in the world of embedded architectures, many questions emerge such as efficiency, flexibility and programming model. This panel will presents a "bake-off" between a number of solutions that have emerged over the recent years. Keywords: Wireless architectures, data communications, implementation platforms 
DAC05, pages 353-358

\title{
Design Methodology for IC Manufacturability Based on Regular Logic-Bricks
}

V. Kheterpal, V. Rovner, T.G. Hersan, D. Motiani, Y. Takegawa, A.J. Strojwas, L. Pileggi

Carnegie Mellon University, Pittsburgh, PA

\begin{abstract}
Implementing logic blocks in an integrated circuit in terms of repeating or regular geometry patterns [6,7] can provide significant advantages in terms of manufacturability and design cost [2]. Various forms of gate and logic arrays have been recently proposed that can offer such pattern regularity to reduce design risk and costs $[2,4,9,11,12]$. In this paper, we propose a fullmask-set design methodology which provides the same physical design coherence as a configurable array, but with area and other design benefits comparable to standard cell ASICs. This methodology is based on a set of simple logic primitives that are mapped to a set of logic bricks that are defined by a restrictive set of RET(Resolution Enhancement Technique)-friendly geometry patterns. We propose a design methodology to explore trade-offs between the number of bricks and associated level of configurability versus the required silicon area. Results are shown to compare a design implemented with a small number of regular bricks to an implementation based on a full standard cell library in a 90nm CMOS technology.

Keywords: Integrated Circuits, Regularity, Manufacturability, RET
\end{abstract}

\section{REFERENCES}

[1] A. Koorapaty et al. "Exploring Logic Block Granularity for Regular Fabrics", Proceedings of the Design, Automation and Test in Europe Conference (DATE), Feb. 2004.

[2] L. Pileggi, H. Schmit, A. J. Strojwas et al., "Exploring regular fabrics to optimize the performance-cost tradeoff". Proceedings of the ACM/IEEE DAC, June 2003.

[3] http://www.monterey.com/products/dolphin.html.

[4] Fan Mo, Robert K. Brayton, "Whirlpool PLAs: a regular logic structure and their synthesis", Proceedings of the 2002 IEEE/ACM ICCAD

[5] M. A. Harrison, Introduction to Switching and Automata Theory, McGraw-Hill, 1965.

[6] M. Palusinski, A. J. Strojwas and W. Maly, "Regularity in Physical Design", GSRC Workshop, Las Vegas, NV, June 17-18, 2001

[7] A. J. Strojwas, "Process-Design Interaction Modeling Based Design for Manufacturability", Tutorial, Design Automation Conference, June 2003.

[8] Yamashita, S. et al., "Pass-transistor/CMOS Collaborated Logic: The Best of Both Worlds", VLSI Circuits, 1997. Digest of Technical Papers.

[9] Yajun Ran and Malgorzata Marek-Sadowska, "On Designing Via-Configurable Cell Blocks for Regular Fabrics", Design Automation Conference (DAC) 2004.

[10] V. Kheterpal, A. J. Strojwas, L. Pileggi, "Routing Architecture Exploration for Regular Fabrics", Proceedings of the ACM/IEEE DAC, June 2004.

[11] T. Okamoto, T.Kimoto, N. Maeda, "Design Methodology and Tools for NEC Electronics' Structured ASIC ISSP", Proceedings of the ISPD, April 2004.

[12] D. Sherlekar, "Design Considerations for Regular Fabrics", Proceedings of the International Symposium on Physical Design (ISPD), April 2004.

[13] K.-C. Wu, Y.-W. Tsai, "Structured ASIC, Evolution or Revolution?", Proceedings of the International Symposium on Physical Design (ISPD), April 2004. 


\author{
Jie Yang§†, Luigi Capodieci§ and Dennis Sylvester $†$ \\ $\S$ Advanced Micro Devices, Sunnyvale, CA \\ $\uparrow$ EECS Dept., University of Michigan, Ann Arbor, MI
}

\begin{abstract}
While performance specifications are verified before sign-off for a modern nanometer scale design, extensive application of optical proximity correction substantially alters the layout introducing systematic variations to the simulated and verified performance. As a result, actual on-silicon chip performance is quite different from sign-off expectations. This paper presents a new methodology to provide better estimates of on-silicon performance. The technique relies on the extraction of residual OPC errors from placed and routed full chip layouts to derive actual (i.e., calibrated to silicon) $\mathrm{CD}$ values that are then used in timing analysis and speed path characterization. This approach is applied to a state-of-the-art microprocessor and contrasted with traditional design flow practices where ideal (i.e., drawn) Lgate values are employed, leading to a subsequent lack of predictive power. We present a platform for diagnosing and improving OPC quality on gates with specific functionality such as critical gates or matching transistors. Furthermore, with more accurate timing analysis we highlight the necessity of a postOPC verification embedded design flow, by showing substantial differences in the Si-based timing simulations in terms of significant reordering of speed path criticality and a $36.4 \%$ increase in worst-case slack. Extensions of this methodology to multi-layer extraction and timing characterization are also proposed.
\end{abstract}

Keywords: OPC, layout, process CD, design flow

\title{
REFERENCES
}

[1] S. R. Nassif, "Modeling and Forecasting of Manufacturing Variations", Proc. Fifth International Workshop on Statistical Metrology, 2000, pp. 3-10.

[2] S. T. Ma, A. Keshavarzi, V. De, and J. R. Brews, "A Statistical Model for Extracting Geometric Sources of Transistor Performance Variation", IEEE Transactions on Electron Devices, 51(1), 2004, pp. 36-41.

[3] M. Orshansky, L. Milor, and C. Hu, "Characterization of Spatial Intrafield Gate CD Variability, Its Impact on Circuit Performance, and Spatial Mask-Level Correction", IEEE Transactions on Semiconductor Manufacturing, 17(1), 2004, pp. 2-11.

[4] C. Visweswariah, "Death, Taxes and Failing Chips", Proc. Design Automation Conference, 2003, pp. 343-347.

[5] A. B. Agrawal, D. Blaauw, V. Zolotov, and S. Vrudhula, "Statistical timing analysis using bounds and selective enumeration", Proc. Design Automation Conference, 2003, pp. 348-353.

[6] M. Orshansky and K. Keutzer, "A General Probabilistic Framework for Worst Case Timing Analysis", Proc. Design Automation Conference, 2002, pp. 556-561.

[7] S. Postnikove and S. Hector, "ITRS CD Error Budgets: Proposed Simulation Study Methodology", May 2003.

[8] M. Orshansky, L. Milor, P. Chen, K. Keutzer and C. Hu, "Impact of Spatial Intrachip Gate Length Variability on the Performance of high-Speed Digital Circuits", IEEE Transactions on Computer Aided Design of Integrated Circuits and Systems, 2002, pp. 544-553.

[9] L. Chen, L. Milor, C. Ouyang, W. Maly, and Y. Peng, "Analysis of the Impact of Proximity Correction Algorithms on Circuit Performance", IEEE Transactions on Semiconductor Manufacturing, 12(3), 1999, pp. 313322.

[10] B. Stine, D. Boning, J. Chung, D. Ciplickas, and J. Kibarian, “ Simulating the Impact of Poly-CD Wafer-Level and Die-Level Variation On Circuit Performance", Proc. Second International Workshop on Statistical Metrology, 1997, pp. 24-27.

[11] P. Gupta and F.L. Heng, "Toward a Systematic-Variation Aware Timing Methodology", Proc. Design Automation Conference, 2004, pp. 321-326. 


$$
\begin{gathered}
\text { Self-Compensating Design for Focus Variation } \\
\text { Puneet } \text { Gupta }^{\dagger} \text {, Andrew B. Kahng }{ }^{\dagger} \text {, Youngmin Kim }{ }^{\ddagger} \text {, Dennis Sylvester } \\
{ }^{\dagger} \text { Blaze DFM Inc.,Sunnyvale CA, } \\
{ }^{\ddagger} \text { EECS Department, University of Michigan at Ann Arbor }
\end{gathered}
$$

\begin{abstract}
Process variations have become a bottleneck for predictable and high-yielding IC design and fabrication. Linewidth variation $(\Delta \mathrm{L})$ due to defocus in a chip is largely systematic after the layout is completed, i.e., dense lines "smile" through focus while isolated (iso) lines "frown". In this paper, we propose a design flow that allows explicit compensation of focus variation, either within a cell (self-compensated cells) or across cells in a critical path (self-compensated design). Assuming that iso and dense variants are available for each library cell, we achieve designs that are more robust to focus variation. Design with a self-compensated cell library incurs $\sim 11-12 \%$ area penalty while compensating for focus variation. Across-cell optimization with a mix of dense and iso cell variants incurs $\sim 6-8 \%$ area overhead compared to the original cell library, while meeting timing constraints across a large range of focus variation (from 0 to $0.4 \mathrm{um}$ ). A combination of original and iso cells provides an even better self-compensating design option, with only $1 \%$ area overhead. Circuit delay distributions are tighter with self-compensated cells and self-compensated design than with a conventional design methodology.

Keywords: Variation, Layout, Focus, ACLV, Manufacturability, Compensation
\end{abstract}

\title{
REFERENCES
}

[1] Y. Cao, et al., "Design Sensitivities to Variability: Extrapolations and Assessments in Nanometer VLSI", Proc. ASIC/SOC, 2002, pp. 411-415.

[2] S. R. Nassif, "Design for Variability in DSM Technologies", Proc. ISQED 2000, pp. 451-454.

[3] S. R. Nassif, "Within-Chip Variability Analysis", Proc. IEDM, 1998, pp. 283-286.

[4] M. Orshansky, L. Milor, P. Chen, K. Keutzer, C. Hu, "Impact of Systematic Spatial Intra-Chip Gate Length Variability on Performance of High-Speed Digital Circuits", ICCAD, 2000, pp. 62-67.

[5] P. Gupta and A. B. Kahng, "Manufacturing-Aware Physical Design", ICCAD, 2003, pp. 681-687.

[6] P. Gupta and H. Fook-Luen, "Toward a systematic-variation aware timing methodology," Proc. DAC, 2004, pp. 321-326.

[7] Calibre version 2004.1_7.33, http://www.mentor.com.

[8] "International Technology Roadmap for Semiconductors 2003," http://public.itrs.net/Files/2003ITRS/ Home2003.htm.

[9] T. Yorick, et al, "ArF imaging with off-axis illumination and subresolution assist bars: a compromise between mask constraints and lithographic process constraints," Proc. SPIE, 2002, vol. 4691, pp. 1522-1529.

[10] A. J. Lori, T. R. Michael, D. Jason, and J. Christiane, "Effect of scattering bar assist features in 193-nm lithography," Proc. SPIE, 2002, vol. 4691, pp. 861-870.

[11] Autochar - Automates the characterization of digital circuits, http://directory.fsf.org/design/cad/autochar.html.

[12] Design Compiler version V-2003.12, http://www.synopsys.com.

[13] A. B. Kahng and Y. C. Pati, "Subwavelength Lithography and its Potential Impact on Design and EDA", Proc.

DAC, 1999, pp. 799-804.

[14] L. W. Liebmann, S. M. Mansfield, A. K. Wong, M. A. Lavin, W. C. Leipold, T.G. Dunham, "TCAD Development for Lithography Resolution Enhancement", IBM J. RES. \& DEV, vol. 45, no. 5, 2001.

[15] S. Sirichotiyakul, et al., "Stand-by power minimization through simultaneous threshold voltage selection and circuit sizing" Proc. DAC, 1999, pp. 436-441.

[16] F. Brglez and H. Fujiwara, "A neutral netlist of 10 combinational benchmark circuits and a target translator in Fortran”, Proc. ISCAS, May 1989, pp. 695-698. 


\title{
RADAR: RET-Aware Detailed Routing Using Fast Lithography Simulations
}

Joydeep Mitra, Peng Yu, David Z. Pan

ECE Department, University of Texas at Austin, Austin, TX

\begin{abstract}
This paper attempts to reconcile the growing interdependency between nanometer lithography and physical design. We first introduce the concept of lithography hotspots and the edge placement error (EPE) map to measure the overall printability and manufacturing effort. We then adapt fast lithography simulation models to generate EPE map. Guided by EPE map, we develop effective RET-aware detailed routing (RADAR) techniques that can handle full-chip capacity to enhance the overall printability while maintaining other design closure. RADAR is implemented in an industry strength detailed router, and tested using some $65 \mathrm{~nm}$ designs. Our experimental results show that we can achieve up to $40 \%$ EPE reduction with reasonable CPU time.
\end{abstract}

Keywords: DFM, RET, OPC, detailed routing, lithography

\section{REFERENCES}

[1] L. W. Liebmann, "Layout impact of resolution enhancement techniques: impediment or opportunity?", Int. Symp. on Physical Design, 2003.

[2] F. M. Schellenberg, "Resolution enhancement technology: the past, the present and extension for the future". SPIE Microlithography Symposium, 2004.

[3] P. Gupta and A. B. Kahng, "Manufacturing-aware physical design," ICCAD, pp. 681-687, 2003.

[4] H. K.-S. Leung, "Advanced routing in changing technology landscape," Int. Symp. on Physical Design, pp. 118$121,2003$.

[5] A. B. Kahng, "Research directions for coevolution of rules and routers," in Proc. Int. Symp. on Physical Design, pp. 122-125, 2003.

[6] L. Scheffer, "Physical CAD changes to incorporate design for lithography and manufacturability," ASPDAC, Jan. 2004.

[7] M. Lavin, F.-K. Luen, and G. Northrop, “Backend CAD flows for 'Restrictive Design Rules'”, ICCAD, 2004.

[8] PROLITH (version 8.0), KLA-Tencor Corporation.

[9] SOLID-CTM (version 6.4.1), Sigma-C Software.

[10] L.-D. Huang and D. F. Wong, "Optical proximity correction (OPC)-friendly maze routing," DAC, 2004.

[11] Y.C. Pati, A.A. Ghazanfarian, and R.F. Pease, "Exploiting structure in fast aerial image computation for IC patterns", IEEE Trans. Semi. Mfg., Feb 1997.

[12] J. Stirniman and M. Rieger, "Fast proximity correction with zone sampling", in Proc. SPIE Symposium on Microlithography, vol. 2197, pp 294-301, 1994.

[13] N. B. Cobb, Fast Optical and process Proximity Correction Algorithms for Integrated Circuit Manufacturing, Ph.D. Thesis, UC Berkeley, 1998.

[14] Blast-Fusion, Magma Design Automation. 


\title{
BDD Representation for Incompletely Specified Multiple-Output Logic Functions and Its Applications to Functional Decomposition
}

\author{
Tsutomu Sasao and Munehiro Matsuura \\ Department of Computer Science and Electronics, Kyushu Institute of Technology, \\ Iizuka, Japan
}

\begin{abstract}
A multiple-output function can be represented by a binary decision diagram for characteristic function (BDD for $\mathrm{CF}$ ). This paper presents a new method to represent multiple-output incompletely specified functions using BDD for CF. An algorithm to reduce the widths of BDD for CFs is presented. This method is useful for decomposition of incompletely specified multiple-output functions. Experimental results for radix converters, adders and a multiplier show that this method is useful for the synthesis of LUT cascades. This data structure is also useful to three-valued logic simulation.
\end{abstract}

Keywords: Incompletely Specified Function, BDD, Characteristic function, Cascade, Code converter

\section{REFERENCES}

[1] R. L. Ashenhurst, "The decomposition of switching functions," International Symposium on the Theory of Switching, pp. 74-116, April 1957.

[2] P. Ashar and S. Malik, "Fast functional simulation using branching programs," Inter. Conf. on CAD, pp. 408412, Nov. 1995.

[3] S. Chang, D. Cheng, and M. Marek-Sadowska, "Minimizing ROBDD size of incompletely specified multiple output functions," In European Design \& Test Conf., pp. 620-624, 1994.

[4] K. Cho and R. E. Bryant, "Test pattern generation for sequential MOS circuits by symbolic fault simulation," Design Automation Conference, pp. 418-423, June 1989.

[5] M. R. Garey and D. S. Johnson, Computers and Intractability, Freeman, San Francisco, 1979.

[6] Y. Hong, P. Beerel, J. Burch, and K. McMillan, "Safe BDD minimization using don't cares," Design Automation Conference, pp. 208-213, 1997.

[7] I. Koren,Computer Arithmetic Algorithms (2nd Edition), A. K. Peters, Natick, MA, 2002.

[8] Y-T. Lai, M. Pedram and S. B. K. Vrudhula, "BDD based decomposition of logic functions with application to FPGA synthesis", Design Automation Conference, 1993.

[9] S. Minato, N. Ishiura, and S. Yajima, "Shared binary decision diagram with attributed edges for efficient Boolean function manipulation," Design Automation Conference, pp. 52-57, June 1990.

[10] A. Mishchenko and T. Sasao, "Encoding of Boolean functions and its application to LUT cascade synthesis," International Workshop on Logic and Synthesis 2002, pp. 115-120, New Orleans, Louisiana, June 4-7, 2002.

[11] K. Nakamura, and T. Sasao, et.al "Programmable logic device with an 8-stage cascade of 64K-bit asynchronous SRAMs," Cool Chips VIII, IEEE Symposium on Low-Power and High-Speed Chips, April 20-22, 2005, Yokohama, Japan (to be published).

[12] R. Rudell, "Dynamic variable ordering for ordered binary decision diagrams," ICCAD-93, pp. 42-47, 1993.

[13] T. Sasao, "FPGA design by generalized functional decomposition," In Logic Synthesis and Optimization, Kluwer Academic Publisher, pages 233-258, 1993.

[14] T. Sasao and M. Matsuura, "A method to decompose multiple-output logic functions," Design Automation Conference, pp. 428-433, San Diego, June 2-6, 2004.

[15] T. Sasao, "Radix converters: Complexity and implementation by LUT cascades," International Symposium on Multiple-Valued Logic, May 2005 (to be published).

[16] Available at http://www.lsi-cad.com/dac2005

[17] M. Sauerhoff and I. Wegener, "On the complexity of minimizing the OBDD size for incompletely specified functions," IEEE Transactions on TCAD, Vol. 15, No. 11, pp. 1435-1437, 1996.

[18] C. Scholl, "Multi-output functional decomposition with exploitation of don't cares," In Design Automation and Test Europe, pp. 743-748, Feb. 1998.

[19] T. R. Shiple, R. Hojati, A. L. Sangiovanni-Vincentelli, and R. K. Brayton, "Heuristic minimization of BDDs using don’t cares," Design Automation Conference, pp. 225-231, 1994. 
[20] W. Wan and M. A. Perkowski, "A new approach to the decomposition of incompletely specified functions based on graph-coloring and local transformations and its application to FPGA mapping," IEEE EURO-DAC'92, pp. 230-235, Hamburg, Sept. 7-10, 1992. 


\title{
A New Canonical Form for Fast Boolean Matching in Logic Synthesis and Verification
}

Afshin Abdollahi, Massoud Pedram

University of Southern California

\begin{abstract}
An efficient and compact canonical form is proposed for the Boolean matching problem under permutation and complementation of variables. In addition an efficient algorithm for computing the proposed canonical form is provided. The efficiency of the algorithm allows it to be applicable to large complex Boolean functions with no limitation on the number of input variables as apposed to previous approaches, which are not capable of handling functions with more than seven inputs. Generalized signatures are used to define and compute the canonical form while symmetry of variables is used to minimize the computational complexity of the algorithm. Experimental results demonstrate the efficiency and applicability of the proposed canonical form.
\end{abstract}

General Terms: Algorithms, Design, Verification

\section{References}

[1] G. De Micheli, Synthesis and Optimization of Digital Circuits, McGraw-Hill, 1994.

[2] L. Benini and G. De Micheli, "A survey of Boolean matching techniques for library binding," ACM Trans. Design Automation of Electronic Systems, vol. 2, no. 3, pp. 193-226, July 1997.

[3] M. A. Harrison, Introduction to Switching and Automata Theory, McGraw-Hill, 1965.

[4] J. Mohnke, P. Molitor, and S. Malik, "Limits of using signatures for permutation independent Boolean comparison," Proc. of ASP Design Automation Conf., pp. 459-464, 1995.

[5] J. R. Burch and D. E. Long, "Efficient Boolean function matching," in Proc. Int. Conf. on Computer-Aided Design, pp. 408-411, Nov. 1992.

[6] Q. Wu, C. Y. R. Chen, and J. M. Acken, "Efficient Boolean matching algorithm for cell libraries," Proc. IEEE Int. Conf. on Computer Design, pp. 36-39, Oct. 1994.

[7] U. Hinsberger and R. Kolla, "Boolean matching for large libraries," Proc. of Design Automation Conf., pp. 206211, June 1998.

[8] D. Debnath and T. Sasao, "Fast Boolean matching under permutation using representative," Proc. ASP Design Automation Conf., pp. 359-362, Jan. 1999.

[9] J. Ciric and C. Sechen, "Efficient canonical form for Boolean matching of complex functions in large libraries," IEEE Trans. Computer-Aided Design of Integrated Circuits and Systems, vol. 22, no. 5, pp. 535-544, May 2003.

[10] D. Debnath and T. Sasao, "Efficient computation of canonical form for Boolean matching in large libraries," Proc. ASP Design Automation Conf., pp. 591-596, Jan. 2004.

[11] C. R. Edwards and S. L. Hurst, "A digital synthesis procedure under function symmetries and mapping methods", IEEE Trans. Comp., Vol. C-27, No. 11, pp. 985-997, NOV. 1978. 


\title{
Effective Bounding Techniques For Solving Unate and Binate Covering Problems
}

\author{
Xiao Yu Li ${ }^{1}$, Matthias F. Stallmann ${ }^{2}$, Franc Brglez ${ }^{2}$ \\ ${ }^{1}$ Amazon, Seattle WA, USA \\ ${ }^{2} \mathrm{NC}$ State University, Raleigh NC, USA
}

\begin{abstract}
Covering problems arise in many areas of electronic design automation such as logic minimization and technology mapping. An exact solution can critically impact both size and performance of the devices being designed. This paper introduces eclipse, a branch-and-bound solver that can solve many covering problems orders of magnitude faster than existing solvers. When used in place of the default covering engine of a well-known logic minimizer, eclipse makes it possible to find, in less than six minutes, true minima for three benchmark problems that have eluded exact solutions for more than a decade.
\end{abstract}

Keywords: covering, branch and bound, satisfiability, unate, binate

\section{REFERENCES}

[1] R. Rudell and A.L. Sangiovanni-Vincentelli. Multiple-valued optimization for PLA optimization. IEEE Transactions on CAD/ICAS, CAD-6(5):727-750, September 1987.

[2] G. D. Micheli. Synthesis and Optimization of Digital Circuits. McGraw-Hill Publishers, 1994.

[3] G.D. Hachtel and F. Somenzi. Logic Synthesis and Verification Algorithms. Kluwer Academic Publishers, 1996.

[4] P.C. McGeer, J.V. Sanghavi, R.K. Brayton, and A.L. Sangiovanni-Vincentelli. Espresso-signature: A new exact minimizer for logic functions. In Proceedings of the $30^{\text {th }}$ Design Automation Conference, pages 618-624, 1993.

[5] O. Coudert. On solving covering problems. In Proceedings of the 33rd Design Automation Conference, pages 197-202, 1996.

[6] S. Liao and S. Devadas. Solving covering problems using lpr-based lower bounds. In Proceedings of the 34th Design Automation Conference, pages 117-120, 1997.

[7] E.I. Goldberg, L.P. Carloni, T. Villa, R.K. Brayton, and A.L. Sangiovanni-Vincentelli. Negative thinking in branch-and-bound: the case of unate covering. IEEE Transactions on Computer-Aided Design of Integrated Circuits and Systems, 19:1-16, 2000.

[8] R. Cordone, F. Ferrandi, D. Sciuto, and R. Wolfler. An efficient heuristic approach to solve the unate covering problem. In Proceedings of the Design, Automation and Test in Europe, 2000.

[9] O. Coudert and J.C. Madre. New ideas for solving covering problems. In Proceedings of the 31st Design Automation Conference, 1995.

[10] T. Villa, T. Kam, R.K. Brayton, and A.L. Sangiovanni-Vincentelli. Explict and implicit algorithms for binate covering problems. IEEE Transactions on Computer-Aided Design of Integrated Circuits and Systems, 16:677-691, 1997.

[11] V.M. Manquinho and J.P. Marques-Silva. Search pruning techniques in SAT-based branch-and-bound algorithms for the binate covering problem. IEEE Transactions on Computer-Aided Design of Integrated Circuits and Systems, 21:505-516, 2002.

[12] S. Khanna, M. Sudan, L. Trevisan, and D. P. Williamson. The approximability of constraint satisfaction problems. SIAM J. Comput., 30(6):1863-1920, 2000.

[13] M. Berkelaar. FTP site for lp solve, 2004. Source code is available at ftp://ftp.es.ele.tue.nl/pub/lp-solve.

[14] ILOG. CPLEX Homepage, 2004. Information on CPLEX is available at http://www.ilog.com/products/cplex/.

[15] X. Y. Li. Optimization Algorithms for the Minimum-Cost Satisfiability Problem. PhD thesis, Computer Science, North Carolina State University, Raleigh, N.C., August 2004. This thesis is accessible at http://www.lib.ncsu.edu/theses/-available/etd-10072004-021218/.

[16] D. Chai and A. Kuehlmann. A fast pseudo-Boolean constraint solver. In Proceedings of the 40th Design Automation Conference, pages 830-835, June 2003.

[17] R.L. Rudell. Logic synthesis for VLSI design. Ph.D. Dissertation, Department of EECS, University of California at Berkeley, 1989.

[18] R.E. Gomory. Outline of an algorithm for integer solution to linear programs. Bulletin of the American Mathematical Society, 64:275, 1958.

[19] R.E. Gomory. An algorithm for the mixed integer problem. RM-2537. Santa Monica California: Rand Corporation, 1960. 
[20] B. Selman, H.J. Levesque, and D. Mitchell. A new method for solving hard satisfiability problems. In P. Rosenbloom and P. Szolovits, editors, Proceedings of the Tenth National Conference on Artificial Intelligence, pages 440-446, Menlo Park, California, 1992. AAAI Press.

[21] D.A. McAllester, B. Selman, and H. Kautz. Evidence for invariants in local search. In Proceedings of AAAI/IAAI, pages 321-326, 1997.

[22] F. Glover. Future paths for integer programming and links to artificial intelligence. Computers and Operations Research, 5:533-549, 1986.

[23] F. Brglez, X. Y. Li, and M. F. M. Stallmann. On SAT instance classes and a method for reliable performance experiments with SAT solvers. Ann. Math. Artif. Intell., 43(1):1-34, 2005. 
Operator-based Model-Order Reduction of Linear Periodically Time-Varying Systems

Yayun Wan, Jaijeet Roychowdhury

ECE Department, University of Minnesota, Minneapolis, MN, USA

\begin{abstract}
\end{abstract}
Linear periodically time-varying (LPTV) abstractions are useful for a variety of communication and computer subsystems. In this paper, we present a novel operator-based model-order reduction (MOR) algorithmfor reducing large LPTVsystems to smaller ones, a capability useful for system-level performance analysis. Our procedure is based on generalizing existing matrixbased Krylov-subspace algorithms to arbitrary function-space operators. Practical benefits of our approach include significantly enhanced algorithm and code modularity, compared to previous LPTV-MOR approaches based on a-priori discretization. We demonstrate the use of the proposed technique on several circuit examples.

Keywords: LPTV systems, model-order reduction, operator, modularity

\title{
REFERENCES
}

[1] W. Chauvet, B. Lacaze, D. Roviras, and A. Duverdier. Characterization of a set of invertible LPTV filters using circulant matrices. In Proc. International Conference Acoust. Speach Signal Process., pages VI-45-VI-48, 2003.

[2] L. Daniel and J. Phillips. Model Order Reduction for Strictly Passive and Causal Distributed Systems. In Proc. IEEE DAC, pages 46-51, June 2002.

[3] D.C. McLernon. Relationship between an LPTV system and the equivalent LTI MIMO structure. IEE Proc.-Vis. Image Signal Process., 150(3):133-137, June 2003.

[4] A. Demir. Phase noise in oscillators: Daes and colored noise sources. In Proc. ICCAD, pages 170-177, 1998.

[5] A. Demir, A. Mehrotra, and J. Roychowdhury. Phase Noise in Oscillators: A Unifying Theory and Numerical Methods for Characterization. In Proc. IEEE DAC, pages 26-31, June 1998.

[6] N. Dong and J. Roychowdhury. Automated Extraction of Broadly Applicable Nonlinear Analog Macromodels from SPICE-level Descriptions. In Proc. IEEE CICC, 2004.

[7] E. Gad and M. Nakhla. Model Order Reduction of Nonuniform Transmission Lines Using Integrated Congruence Transform. In Proc. IEEE DAC, pages 238-243, June 2003.

[8] P. Feldmann and R.W. Freund. Efficient linear circuit analysis by Pad'e approximation via the Lanczos process. IEEE Trans. CAD, 14(5):639-649, May 1995.

[9] R. Grimshaw. Nonlinear Ordinary Differential Equations. Blackwell Scientific, 1990.

[10] J. Crols and M. S. J. Steyaert. A 1.5 GHz Highly Linear CMOS Downconversion Mixer. IEEE J. Solid-State Ckts., 30(7):736-742, July 1995.

[11] J. R. Phillips. Projection-Based Approaches for Model Reduction of Weakly Nonlinear, Time-Varying Systems. IEEE Trans. CAD, 22(2):171-187, February 2003.

[12] A. Odabasioglu, M. Celik, and L.T. Pileggi. PRIMA: passive reduced-order interconnect macromodelling algorithm. In Proc. ICCAD, pages 58-65, November 1997.

[13] Q. Yu, J. M. L. Wang, and E. S. Kuh. Passive Multipoint Moment Matching Model Order Reduction Algorithm on Multiport Distributed Interconnect Networks. IEEE Trans. Ckts. Syst. - I: Fund. Th. Appl., 46(1):140-160, January 1999.

[14] J. Roychowdhury. Reduced-order modelling of time-varying systems. IEEE Trans. Ckts. Syst. - II: Sig. Proc., 46(10):1273-1288, November 1999.

[15] P. Vanassche, G. Gielen, and W. Sansen. Time-Varying, Frequency-Domain Modeling and Analysis of PhaseLocked Loops with Sampling Phase-Frequency Detectors. In Design, Automation and Test in Europe Conference and Exhibition, 2003.

[16] Z. Wang, R. Murgai, and J. Roychowdhury. Automated, Accurate Macromodelling of Digital Aggressors for Power and Ground Supply Noise Prediction. In Design, Automation and Test in Europe Conference and Exhibition, volume 2, pages 20824-20829, February 2004. 


\title{
Simulation of the Effects of Timing Jitter in Track-and-Hold and Sample-and-Hold Circuits
}

\author{
V.Vasudevan
}

Department of Electrical Engineering, Indian Institute of Technology-Madras, Chennai, India

\begin{abstract}
In this paper, we analyze the effect of jitter in track and hold circuits. The output spectrum is obtained in terms of the system function of the track and hold. It is a fairly general model in which the effect of input as well as clock jitter can be included. The clock can have an arbitrary duty cycle, so that the circuit could also approximate a sample and hold. Using this model, it is possible to simulate the effects of jitter in a track and hold using a standard circuit simulator. Three cases are analyzed - long term jitter, correlated jitter with exponential autocorrelation and white noise jitter. These results are verified using Monte Carlo simulations.
\end{abstract}

Keywords: Jitter, Sampling circuits

\section{REFERENCES}

[1] Nicola Da Dalt, M.Harteneck, C.Sander, and A.Weisbauer, "On the jitter requirements of the sampling clock for analog-to-digital converters," IEEE Trans. Circuits and Syst.-I, vol. 49, pp. 1354-1360, Sept. 2002 .

[2] M.Shinagawa, Y.Akazawa, and T.Wakimoto, "Jitter analysis of high speed sampling systems," IEEE J. Solid State Circuits, vol. 25, pp. 220-224, Feb. 1990.

[3] A.Demir, A.Mehrotra, and J.Roychowdhury, "Phase noise in oscillators: A unifying theory and numerical methods for characterization," IEEE Trans. Circuits and Syst.-I, vol. 47, pp. 655-674, May 2000.

[4] M.Lax, "Classical noise v. noise in self-sustained oscillators," Physical Review, vol. 160, pp. 290-307, Aug. 1967.

[5] A.V.Balakrishnan, "On the problem of time jitter in sampling," IRE Transactions on Information Theory, pp. 226-236, April 1962.

[6] A.Berkovitz and I. Rusnak, "FFT processing of randomly sampled harmonic signals," IEEE Transactions on Signal Processing, vol. 40, pp. 2816-2819, Nov. 1992.

[7] S.S.Awad, "The effects of accumulated timing jitter on some sine wave measurements," IEEE Trans. Inst. Meas., vol. 44, pp. 945-951, Oct. 1995.

[8] N.J.Kasdin, "Discrete simulations of colored noise and stochastic processes and $1 / f \alpha$ power law noise generation," Proc. IEEE, vol. 83, pp. 802-827, May 1995.

[9] A.V.Oppenheim and R.W.Schafer, Discrete-Time Signal Processing. Prentice Hall, N.J, 3 ed., 1989. 
Scalable Trajectory Methods for On-Demand Analog Macromodel Extraction

\author{
Saurabh K Tiwary, Rob A Rutenbar \\ Carnegie Mellon University, Pittsburgh,PA USA
}

\begin{abstract}
Trajectory methods sample the state trajectory of a circuit as it simulates in the time domain, and build macromodels by reducing and interpolating among the linearizations created at a suitably spaced subset of the time points visited during training simulations. Unfortunately, moving from simple to industrial circuits requires more extensive training, which creates models too large to interpolate efficiently. To make trajectory methods practical, we describe a scalable interpolation architecture, and the first implementation of a complete trajectory "infrastructure" inside a full SPICE engine. The approach supports arbitrarily large training runs, automatically prunes redundant trajectory samples, supports limited hierarchy, enables incremental macromodel updates, and gives 3-10X speedups for larger circuits.
\end{abstract}

Keywords: Circuit, trajectory method, analog, macromodel, SPICE

\title{
REFERENCES
}

[1] M.Takahashi, K.Ogawa, and K.Kundert. VCO jitter simulation and its comparision with measurements. In ASP$D A C, 1999$.

[2] S.K.Tiwary, S.Velu, R.A.Rutenbar, and T.Mukherjee. Pareto optimal modeling for efficient PLL optimization. In Modeling and Simulation of Microsystems, Nanotech, pages 195-198, 2004.

[3] G.G.E.Gielen and R.A.Rutenbar. Computer-aided design of analog and mixed-signal integrated circuits. In Proc. of IEEE, Vol:88 Issue: 12, pages 1825-1854, 2000.

[4] L.T.Pillage and R.A.Rohrer. Asymptotic waveform evaluation. In TCAD, pages 352-366, 1990.

[5] A. Odabasioglu, M. Celik, and L.T. Pileggi. PRIMA: Passive reduced-order interconnect macromodeling algorithm. In TCAD, Vol 17, No 8, pages 645-654, 1998.

[6] P.Wambacq, G.Gielen, and W.Sansen. Interactive symbolic distortion analysis of analogue integrated circuits. EDAC, pages 484-488, 1991.

[7] Joel Phillips. Projection frameworks for model reduction of weakly nonlinear systems. In DAC, pages 184-189. ACM Press, 2000.

[8] Peng Li and L.T.Pileggi. NORM: compact model order reduction of weakly nonlinear systems. In $D A C$, pages 472-477, 2003.

[9] D.Vasilyev, M.Rewienski, and J.White. A TBR-based trajectory piecewise-linear algorithm for generating accurate low-order models for non-linear analog circuits and mems. DAC, pages 490-495, 2003.

[10] Michal Rewienski and Jacob White. A trajectory piecewise-linear approach to model order reduction and fast simulation of nonlinear circuits and micromachined devices. In $T C A D$, pages 155-170, 2003.

[11] Ning Dong and J.Roychowdhury. Automated extraction of broadly applicable nonlinear analog macromodels from SPICE-level descriptions. In CICC, 2004.

[12] Ning Dong and J.Roychowdhury. Piecewise polynomial nonlinear model reduction. In DAC, pages 484-489, 2003.

[13] M.Rewienski and J.White. A trajectory piecewise linear approach to model order reduction and fast simulation of non-linear circuits and micromachined devices. TCAD, pages 155-170, 2003.

[14] T.Quarles. The SPICE3 implementation guide. In UCB/ERL M89/44, April 1989.

[15] R.W.Freund. Krylov-subspace methods for reduced order modeling in circuit simulation. Journal of Computational and Applied Mathematics, 2000.

[16] S.K.Tiwary. Scalable trajectory methods for on-demand analog macromodel extraction. In Phd Thesis (in preparation), CMU, 2005.

[17] Pillage, Rohrer, and Visweswariah. Electronic circuit and system simulation methods. McGraw-Hill, 1995.

[18] A.P.Dempster, N.Laird, and D.Rubin. Maximum-likelihood from incomplete data via the EM algorithm. In $J$. of Royal Statistics Society, B39, 1977.

[19] J.H.Friedman, J.L.Bentley, and R.A.Finkel. An algorithm for finding best matches in logarithmic expected time. In ACM Trans. On Mathematical Software 3(3), pages 209-226, 1977. 
[20] S.Arya, D.M.Mount, N.S.Netanyahu, R.Silverman, and A.Wu. An optimal algorithm for approximate nearest neighbor searching. In ACM-SIAM Symp. on Discrete algorithms, pages 573-582, 1994. 


\title{
Cognitive Radio Techniques for Wide Area Networks
}

\author{
William Krenik and Anuj Batra \\ Texas Instruments Incorporated, Dallas, Texas, USA
}

\begin{abstract}
The cellular wireless market has begun the transition to data centric services including high speed internet access, video, high quality audio, and gaming. Communications technology can meet the need for very high data link speeds, and can also improve network throughput, but dramatically more spectrum will be needed to provide ubiquitous wireless data service. Cognitive radio is a new technology that allows spectrum to be dynamically shared between users. It offers the potential to dramatically change the way spectrum is used in systems and to substantially increase the amount of spectrum available for wireless communications. This paper introduces cognitive radio and explains the promise, possible operating modes, and benefits it may offer.
\end{abstract}

Keywords: Cognitive radios, unlicensed spectrum, unlicensed wide area network

\section{REFERENCES}

[1] Federal Communications Commission, "Spectrum Policy Task Force Report," ET Docket No. 02-135, Nov. 2002.

[2] W. Lehr, "The Economic Case for Dedicated Unlicensed Spectrum Below 3GHz," New America Foundation, Spectrum Policy Program White Paper, Spectrum Series Issue Brief \#16, July 2004.

[3] Federal Communications Commission, "Unlicensed Operation in the TV Broadcast Bands," ET Docket No. 04186, 2004.

[4] J. Mitola, III, "Cognitive Radio for Flexible Mobile Multimedia Communications," Mobile Multimedia Communications, 1999. IEEE International Workshop, page 3.

[5] B. Krenik and C. Panasik, "The Potential for Unlicensed Wide Area Networks," Wireless Advanced Architectures Group, Texas Instruments White Paper, November 2004.

[6] J. Reed, L. DaSilva, J. Suris, L. Morales, "Potential for Unlicensed Wide Area Networks Using Cognitive Radios and Available Resource Maps," Mobile and Portable Radio Research Group, Bradley Department of Electrical and Computer Engineering, Virginia Tech, White Paper, Feb. 5, 2005.

[7] J. Neel, R.M. Buehrer, J.H. Reed and R.P. Gilles, "Game Theoretic Analysis of a Network of Cognitive Radios," Midwest Symposium on Circuits and Systems 2002.

[8] T. W. Rondeau, C. J. Rieser, B. Le, and C. W. Bostian, "Cognitive Radios with Genetic Algorithms: Intelligent Control of Software Defined Radios," Proc. SDR04, Phoenix, 2004, pp. C-3 - C-8.

[9] J. Mitola, III, “Cognitive INFOSEC," IEEE MTT-S Digest, 2003, page 1051. 


\title{
MIMO Technology for Advanced Wireless Local Area Networks
}

Jeffrey M. Gilbert, Won-Joon Choi, Qinfang Sun

Atheros Commications, Inc., Sunnyvale, CA 94085

\author{
ABSTRACT \\ This paper first gives a brief introduction to Multiple Input Multiple Output (MIMO) wireless \\ communication systems. Various architectures of MIMO systems and corresponding features are \\ discussed, including those proposed for the IEEE 802.11n standard. The impact on chip area and \\ required data processing rates is then presented. \\ Keywords: MIMO, 802.11n, Wireless, Networking

\section{REFERENCES} \\ [1] W. McFarland, W. J. Choi, A. Tehrani, J. Gilbert et. al. A WLAN Soc for Video Applications Including \\ Beamforming and Maximum Ratio Combining. ISSCC, 2005. \\ [2] S. Mehta, D. Weber, M. Terrovitis, K. Onodera et. al. An 802.11g WLAN SoC. ISSCC, 2005. \\ [3] A. Pauraj, R. Nabar and D. Gore. Introduction to Space-Time Wireless Communications. Cambridge University \\ Press, Cambridge, 2003. \\ [4] http://www.atheros.com \\ [5] ftp://ftp.802wirelessworld.com/11/03/11-03-0813-12-000nfunctional-requirements.doc \\ [6] http://www.tgnsync.org \\ [7] http://www.wwise.org
}


DAC05, pages 416-420

\title{
RF MEMS in Wireless Architectures
}

Clark T.-C. Nguyen

DARPA/MTO, Arlington, Virginia

(On leave from the University of Michigan, Ann Arbor, Michigan)

\begin{abstract}
Micromechanical (or " $\mu$ mechanical") communication circuits fabricated via IC-compatible MEMS technologies and capable of low-loss filtering, mixing, switching, and frequency generation, are described with the intent to miniaturize wireless transceivers. Possible MEMSbased receiver front-end architectures are then presented that use these micromechanical circuits in large quantities to enhance robustness and substantially reduce power consumption. Among the more aggressive architectures proposed are one based on a $\mu$ mechanical RF channel-selector and one featuring an all-MEMS RF front-end.

Keywords: RF MEMS, quality factor, micromechanical circuit, RF front end, resonator, switch, inductor, capacitor
\end{abstract}

\section{REFERENCES}

[1] C. T.-C. Nguyen, 2004 IEEE Custom Integrated Ckts. Conf. (CICC), Orlando, Florida, Oct. 3-6, 2004, pp. $257-$ 264.

[2] C. T.-C. Nguyen, 2000 Bipolar/BiCMOS Ckts. and Tech. Mtg (BCTM), Sept. 25-26, 2000, pp. 142-149.

[3] C. T.-C. Nguyen, IEEE Trans. Microwave Theory Tech., vol. 47, no. 8, pp. 1486-1503, Aug. 1999.

[4] C. T.-C. Nguyen, L. P.B. Katehi, and G. M. Rebeiz, Proc. IEEE, vol. 86, no. 8, pp. 1756-1768, Aug. 1998.

[5] Z. J. Yao, et al., IEEE/ASME J. Microelectromech. Syst., pp. 129-134, June 1999.

[6] D. J. Young, et al., 1996 Solid-State Sensor and Actuator Workshop, June 2-6, 1996, pp. 86-89.

[7] J.-B. Yoon, et al., 2000 IEEE Int. Electron Devices Meeting (IEDM), Dec. 11-13, 2000, pp. 489-492.

[8] C. L. Chua, et al., IEEE/ASME J. Microelectromech. Syst., vol. 12, no. 6, pp. 989-995, Dec. 2003.

[9] F. D. Bannon III, et al., IEEE J. Solid-State Circuits, vol. 35, no. 4, pp. 512-526, April 2000.

[10] K. Wang, et al., IEEE/ASME J. Microelectromech. Syst., vol. 9, no. 3, pp. 347-360, Sept. 2000.

[11] M. A. Abdelmoneum, et al., Proceedings, 2003 IEEE MEMS Conf., Kyoto, Japan, Jan. 19.-23, 2003, pp. 698701.

[12] J. Wang, et al., IEEE Trans. Ultrason., Ferroelect., Freq. Contr., vol. 51, no. 12, pp. 1607-1628, Dec. 2004.

[13] J. Wang, et al., 2004 IEEE MEMS Conf., Maastricht, The Netherlands, Jan. 25-29, 2004, pp. 641-644.

[14] S.-S. Li, et al., 2004 IEEE MEMS Conf., Maastricht, Netherlands, Jan. 25-29, 2004, pp. 821-824.

[15] A. Dec, et al., IEEE J. Solid-State Circuits, vol. 35, no. 8, pp. 1231-1237, Aug. 2000.

[16] M. L. Roukes, 2000 Solid-State Sensor and Actuator Workshop, June 4-8, 2000, pp. 367-376.

[17] J. R. Vig, et al., IEEE Trans. Utrason. Ferroelec. Freq. Contr., vol. 46, no. 6, pp. 1558-1565, Nov. 1999.

[18] G. Piazza, et al., 2005 IEEE MEMS Conf., Miami, Florida, Jan. 30 - Feb 3, 2005, pp. 20-23.

[19] Y.-W. Lin, et al., IEEE J. Solid-State Circuits, vol. 39, no. 12, pp. 2477-2491, Dec. 2004.

[20] B. P. Otis and J. M. Rabaey, IEEE J. Solid-State Circuits, vol. 38, no. 7, pp. 1271-1274, July 2003.

[21] A.-C. Wong and C. T.-C. Nguyen, IEEE/ASME J. Microelectromech. Syst., vol. 13, no. 1, pp. 100-112, Feb. 2004.

[22] J.P. Raskin, et al., IEEE/ASME J. Microelectromech. Syst, vol. 9, pp. 528-537, Dec. 2000.

[23] M. U. Demirci and C. T.-C. Nguyen, "A Low Impedance VHF Micromechanical Filter Using Coupled-Array Composite Resonators," to be published in the Technical Digest of TRANSDUCERS'05, Seoul, Korea, June 5-9, 2005.

[24] C. T.-C. Nguyen and R. T. Howe, IEEE J. Solid-State Circuits, vol. 34, no. 4, pp. 440-455, April 1999. 


\title{
Multiplexer Restructuring for FPGA Implementation Cost Reduction
}

Paul Metzgen, Dominic Nancekievill

Altera European Technology Center, High Wycombe, Buckinghamshire, UK

\begin{abstract}
This paper presents a novel synthesis algorithm that reduces the area needed for implementing multiplexers on an FPGA by an average of $18 \%$. This is achieved by reducing the number of Lookup Tables (LUTs) needed to implement multiplexers. The algorithm relies on reimplementing 2:1 multiplexer trees using efficient 4:1 multiplexers. The key to the algorithm's performance lies in exploiting the observation that most multiplexers occur in busses. New optimizations are employed which pay a small cost in logic that is shared across the bus to achieve a reduction in the logic required for every bit of the bus.

Keywords: FPGA, Multiplexers, Restructuring, Recoding, Busses, Logic Optimization, Synthesis

\section{REFERENCES}

[1] A High Performance 32-bit ALU for Programmable Logic. P. Metzgen. Proceedings of the 2004 ACM/SIGDA $12^{\text {th }}$ international symposium on Field Programmable Gate Arrays. Pp 61-70. 2004.

[2] FPGA Performance Benchmarking Methodology, White Paper, www.altera.com

[3] The Stratix Device Handbook (Vol 1). Altera Corporation, 2004.

[4] E. M. Sentovich et al. "SIS: A System for Sequential Circuit Synthesis." Technical Report, University of California at Berkeley, 1992, Memorandum No. UCB/ERL M92/41

[5] J. Cong and Y. Ding, "FlowMap: An Optimal Technology Mapping Algorithm for Delay Optimization in Lookup-Table based FPGA Designs", IEEE Trans. CAD Vol 13, No 1, pp. 1-12, 1994.

[6] V. Manohararajah, S.D. Brown and Z. Vranesic, "Heuristics for Area Minimization in LUT-Based FPGA Technology Mapping", in Proc. of the Int'l Workshop on Logic Synthesis 2004

[7] D. Lewis et al, C. Wysocki and R. Cliff, "The Stratix Routing and Logic Architecture", in Proc. ACM/SIGDA Int'l Symposium on FPGAs (FPGA 2003), pp.12-20, 2003

[8] David Lewis et al, "The Stratix-II Routing and Logic Architecture". 2005 Int'l Symposium on FPGAs (FPGA 2005)
\end{abstract}




\title{
FPGA Technology Mapping: A Study of Optimality
}

\author{
Andrew Ling ${ }^{1}$, Deshanand P. Singh ${ }^{2}$, Stephen D. Brown ${ }^{2}$ \\ ${ }^{1}$ Department of Electrical and Computer Engineering, University of Toronto, Toronto, Canada \\ ${ }^{2}$ Altera Corporation Toronto Technology Centre, Toronto, Canada
}

\begin{abstract}
This paper attempts to quantify the optimality of FPGA technology mapping algorithms. We develop an algorithm, based on Boolean satisfiability (SAT), that is able to map a small subcircuit into the smallest possible number of lookup tables (LUTs) needed to realize its functionality. We iteratively apply this technique to small portions of circuits that have already been technology mapped by the best available mapping algorithms for FPGAs. In many cases, the optimal mapping of the subcircuit uses fewer LUTs than is obtained by the technology mapping algorithm. We show that for some circuits the total area improvement can be up to $67 \%$.
\end{abstract}

Keywords: Boolean Satisfiability, Resynthesis, Optimization, Cone, FPGA, Lookup Table

\section{REFERENCES}

[1] Altera. Component selector guide ver 14.0, 2004.

[2] R. K. Brayton and G. D. H. et al. VIS: a system for verification and synthesis. In Proceedings of the Eighth International Conference on Computer Aided Verification CAV, pages 428-432, 1996.

[3] D. Chai, J. Jiang, Y. Jiang, Y. Li, A. Mishchenko, and R. Brayton. MVSIS 2.0 Programmer's Manual, UC Berkeley. Technical report, 2003.

[4] J. Cong and Y. Ding. On area/depth trade-off in LUT-based FPGA technology mapping. In Design Automation Conference, pages 213-218, 1993.

[5] J. Cong, J. Peck, and Y. Ding. RASP: A general logic synthesis system for SRAM-based FPGAs. In FPGA, pages 137-143, 1996.

[6] J. Cong, C. Wu, and Y. Ding. Cut ranking and pruning: enabling a general and efficient fpga mapping solution. In Proceedings of the 1999 ACM/SIGDA seventh international symposium on Field programmable gate arrays, pages 29-35. ACM Press, 1999.

[7] J. Cong, $\mathrm{C}$. $\mathrm{Wu}$, and $\mathrm{Y}$. Ding. Cut ranking and pruning: Enabling a general and efficient FPGA mapping solution. In FPGA, pages 29-35, 1999.

[8] F. Corno, M. Reorda, and G. Squillero. RT-level ITC 99 benchmarks and first ATPG results, 2000.

[9] L. L. C. M. R. M. A. S. H. S. P. R. S. R. K. B. E. M. Sentovich, K. J. Singh and A. Sangiovanni-Vincentelli. SIS: A system for sequential circuit synthesis. Technical report, 1992.

[10] A. Farrahi and M. Sarrafzadeh. Complexity of the Lookup-Table Minimization Problem for FPGA Technology Mapping. IEEE Transactions on Computer-Aided Design of Integrated Circuits and Systems, 13(11):1319-1332, 1994.

[11] R. J. Francis, J. Rose, and K. Chung. Chortle: a technology mapping program for lookup table-based field programmable gate arrays. In Proceedings of the 27th ACM/IEEE conference on Design automation, pages 613-619. ACM Press, 1990.

[12] W. L. Jason Cong, Joey Y. Lin. Spfd-based global rewiring. In Proceeding of International Symposium on FPGAs, pages 77-84, February 2002.

[13] K. Keutzer. Dagon: Technology binding and local optimization by dag matching. In DAC, pages 341-347, 1987.

[14] T. Larrabee. Test Pattern Generation Using Boolean Satisfiablity. IEEE Transactions on Computer-Aided Design, 11(1):6-22, 1992.

[15] I. Levin and R. Y. Pinter. Realizing Expression Graphs using Table-Lookup FPGAs. In Proceedings of the European Design Automation Conference, pages 306-311, 1993.

[16] M. W. Moskewicz, C. F. Madigan, Y. Zhao, L. Zhang, and S. Malik. Chaff: Engineering an Efficient SAT Solver. In Proceedings of the 38th Design Automation Conference (DAC'01), 2001.

[17] M. D. F. Schlag, J. Kong, and P. K. Chan. Routability-driven techology mapping for lookup-table-based fpgas. In Proceedings of the 1991 IEEE International Conference on Computer Design on VLSI in Computer \& Processors, pages 86-90. IEEE Computer Society, 1992. 
[18] Xilinx. Virtex-ii complete data sheet ver 3.3, 2004.

[19] S. Yamashita, H. Sawada, and A. Nagoya. A new method to express functional permissibilities for LUT based FPGAs and its applications. In ICCAD, pages 254-261, 1996.

[20] S. Yang. Logic synthesis and optimization benchmarks user guide version, 1991. 


\title{
Incremental Retiming for FPGA Physical Synthesis
}

Deshanand P. Singh, Valavan Manohararajah, Stephen D. Brown

Altera Corporation, Toronto Technology Center, Toronto, CANADA

\begin{abstract}
In this paper, we present a new linear-time retiming algorithm that produces near-optimal results. Our implementation is specifically targeted at Altera's Stratix [1] FPGA-based designs, although the techniques described are general enough for any implementation medium. The algorithm is able to handle the architectural constraints of the target device, multiple timing constraints assigned by the user and implicit legality constraints. It ensures that register moves do not create asynchonous problems such as creating a glitch on a clock/reset signal.
\end{abstract}

Keywords: Retiming, Physical Synthesis, FPGA

\section{REFERENCES}

[1] Altera. Altera Databook.

[2] K. Eckl, J.C. Madre, P. Zepter and C. Legl. A Practical Approach to Multiple-Class Retiming. DAC, 1999.

[3] C. Leiserson, F. Rose, and J. Saxe. Optimizing synchronous circuitry. Journal of VLSI and Computer Systems, pages 41-67, 1983.

[4] C. Leiserson and J. Saxe. Retiming synchronous circuitry. Algorithmica, 6(1):5-35, 1991.

[5] D. Lewis, V. Betz et al, The Stratix Routing and Logic Architecture. FPGA 2003.

[6] N. Maheshwari and S. S. Sapatnekar. Efficient retiming of large circuits. IEEE Transactions on VLSI Systems, 6(1):74-83, 1998.

[7] N. Shenoy and R. Rudell. Efficient implementation of retiming. In ICCAD 1994, pages 226-233, November 1994.

[8] D. Singh and S. Brown Incremental Placement for Layout-Driven Optimizations on FPGAs. ICCAD 2002.

[9] D. Singh and S. Brown. Integrated Retiming and Placement for FPGAs. FPGA 2002.

[10] P. Suaris, D. Wang and N. Chou. Smart Move: A placement-aware retiming and replication method for Field Programmable Gate Arrays. ASIC 2003.

[11] B. van Antwerpen, M. Hutton, G. Baeckler and R. Yuan. A Safe and Complete Gate-Level Register Retiming Algorithm. In IWLS 2003, pages 140-147, 2003. 


\begin{abstract}
Previous research has shown both theoretically and practically that simulated annealing can greatly benefit from the incorporation of an adaptive range limiting window to control the acceptance ratio of swaps during placement. However, the implementation of such a system is not necessarily obvious. Existing range limiting techniques have several fundamental shortcomings when dealing with both standard island-style FPGAs and more exotic architectures. In this paper we discuss the nature of these problems and present a new algorithm that attempts to deal with these issues.

Keywords: Reconfigurable logic, placement, simulated annealing, windowing, range limiting, architecture-adaptive

\section{References}

[1] Betz, Vaughn and Jonathon Rose. "VPR: A New Packing, Placement and Routing Tool for FPGA Research." International Workshop on Field Programmable Logic and Applications, 1997: 213-22.

[2] Betz, Vaughn, Jonathan Rose, and Alexander Marquardt, Architecture and CAD for Deep-Submicron FPGAs, Kluwer Academic Publishers, 1999.

[3] DeHon, A. "Balancing Interconnect and Computation in a Reconfigurable Computing Array (or, why you don't really want 100\% LUT utilization).” International Symposium on Field Programmable Gate Arrays, 1999: $125-34$.

[4] Eguro, K. and S. Hauck, "Issues of Wirelength Cost Models in Routing-Constrained FPGAs", University of Washington, Dept. of EE Technical Report UWEETR-2004-0006, 2004.

[5] Lam, J. and J. M. Delosme, "Performance of a New Annealing Schedule." Proc. 25th Design Automation Conf., 1988, pp. 306-11.

[6] Sharma, A., C. Ebeling, and S. Hauck. "Architecture Adaptive Routability-Driven Placement for FPGAs". Submitted to International Symposium on Field Programmable Logic and Applications, 2005.

[7] Tsu, W., K. Macy, A. Joshi, R. Huang, N. Walker, T. Tung, O. Rowhani, V. George, J. Wawrzynek, and A. DeHon. "HSRA: High-Speed, Hierarchical Synchronous Reconfigurable Array". International Symposium on Field Programmable Gate Arrays, 1999: 125-34.
\end{abstract}


DAC05, pages 445-450

\begin{tabular}{l} 
Word Level Predicate Abstraction and Refinement for Verifying RTL Verilo \\
\hline Himanshu Jain ${ }^{1}$, Daniel Kroening ${ }^{2}$, Natasha Sharygina ${ }^{3}$, Edmund Clarke $^{l}$ \\
${ }^{1}$ CMU SCS, Pittsburgh, PA \\
${ }^{2}$ ETH Z"urich, Switzerland \\
${ }^{3}$ CMU SCS and SEI, Pittsburgh, PA
\end{tabular}

\begin{abstract}
Model checking techniques applied to large industrial circuits suffer from the state space explosion problem. A major technique to address this problem is abstraction. The most commonly used abstraction technique for hardware verification is localization reduction, which removes latches that are not relevant to the property. However, localization reduction fails to reduce the size of the model if the property actually depends on most of the latches. This paper proposes to use predicate abstraction for verifying RTL Verilog, a technique successfully used for software verification. The main challenge when using predicate abstraction is the discovery of suitable predicates. We propose to use weakest preconditions of Verilog statements in order to obtain new predicates during abstraction refinement. This technique has not been applied to circuits before. On benchmarks taken from an industrial microprocessor, we successfully verified safety properties with more than 32,000 latches in the cone of influence. We compare the performance of our technique with a modern model checker that implements localization reduction.
\end{abstract}

Keywords: Predicate Abstraction, Verilog, SAT

\title{
REFERENCES
}

[1] Z. S. Andraus and K. A. Sakallah. Automatic abstraction and verification of Verilog models. In $D A C$, pages 218-223, 2004.

[2] T. Ball, B. Cook, S. Das, and S.K. Rajamani. Refining approximations in software predicate abstraction. In TACAS, pages 388-403, 2004.

[3] T. Ball and S.K. Rajamani. Boolean programs: A model and process for software analysis. Technical Report 2000-14, Microsoft Research, 2000.

[4] A. Biere, A. Cimatti, E. Clarke, and Y. Yhu. Symbolic model checking without BDDs. In TACAS, pages 193207, 1999.

[5] R. Bryant, S. Lahiri, and S. Seshia. Modeling and verifying systems using a logic of counter arithmetic with lambda expressions and uninterpreted functions. In $C A V, 2002$.

[6] P. Chauhan, E.M. Clarke, J. Kukula, S. Sapra, H. Veith, and D. Wang. Automated abstraction refinement for model checking large state spaces using sat based conflict analysis. In FMCAD, 2002.

[7] E. Clarke, O. Grumberg, S. Jha, Y. Lu, and Veith H. Counterexample-guided abstraction refinement. In $C A V$, pages 154-169, 2000.

[8] E. Clarke, O. Grumberg, S. Jha, Y. Lu, and H. Veith. Counterexample-guided abstraction refinement for symbolic model checking. J. $A C M, 50(5), 2003$.

[9] E. Clarke, O. Grumberg, and D.E. Long. Model checking and abstraction. In POPL, 1992.

[10] E. Clarke, O. Grumberg, and D. Peled. Model Checking. MIT Press, 1999.

[11] E. Clarke, H. Jain, and D. Kroening. Predicate Abstraction and Refinement Techniques for Verifying Verilog. Technical Report CMU-CS-04-139, Carnegie Mellon University, 2004.

[12] E. Clarke, D. Kroening, N. Sharygina, and K. Yorav. Predicate abstraction of ANSI-C programs using SAT. Formal Methods In System Design, 25, 2004.

[13] E. Clarke, M. Talupur, and D. Wang. SAT based predicate abstraction for hardware verification. In SAT, 2003.

[14] www-cad.eecs.berkeley.edu/ kenmcmil/smv/.

[15] S. Das and D. Dill. Successive approximation of abstract transition relations. In LICS, 2001. June 2001, Boston, USA.

[16] S. Graf and H. Saidi. Construction of abstract state graphs with PVS. In $C A V$, volume 1254, pages 72-83, 1997.

[17] T. A. Henzinger, R. Jhala, R. Majumdar, and G. Sutre. Lazy abstraction. In POPL, pages 58-70, 2002. 
[18] R.P. Kurshan. Computer-aided verification of coordinating processes: the automata-theoretic approach. Princeton University Press, 1994.

[19] M. Moskewicz, C. Madigan, Y. Zhao, L. Zhang, and S. Malik. Chaff: Engineering an efficient SAT solver. In $D A C$, pages 530-535, 2001.

[20] Kedar S. Namjoshi and Robert P. Kurshan. Syntactic program transformations for automatic abstraction. In $C A V$ 00, number 1855 in LNCS, 2000.

[21] http://nusmv.irst.itc.it/.

[22] http://www.cs.sfu.ca/ loryan/personal.

[23] http://www.sun.com/processors/technologies.html.

[24] D. Wang, P. Ho, J. Long, J. Kukula, Y. Zhu, T. Ma, and R. Damiano. Formal property verification by abstraction refinement with formal, simulation and hybrid engines. In $D A C$, pages 35-40, 2001.

[25] L. Zhang and S. Malik. Extracting small unsatisfiable cores from unsatisfiable boolean formulas. In SAT, 2003. 


\title{
Structural Search for RTL with Predicate Learning
}

G. Parthasarathy, M. K. Iyer, K.T. Cheng, F. Brewer

Dept of Electrical and Computer Engineering, University of California - Santa Barbara Santa Barbara, CA

\begin{abstract}
We present an efficient search strategy for satisfiability checking on circuits represented at the register-transfer-level (RTL). We use the RTL circuit structure by extending concepts from classic automatic test-pattern generation (ATPG) algorithms and interval-arithmetic to guide the search process. We extend the idea of Boolean recursive learning on predicate logic in the RTL using Boolean and interval constraint propagation in the control and data-path of the circuit. This is used as a pre-processing step to derive relations between predicate logic signals that are used to augment the search. We demonstrate experimentally that these methods provide significant improvement over current techniques on sample benchmarks.
\end{abstract}

Keywords: Interval Arithmetic, Learning, Predicate Abstraction, Satisfiability

\section{REFERENCES}

[1] M. Abramovici, M. A. Breuer, and A. D. Friedman. Digital Systems Testing and Testable Design. CS Press, 1st edition, 1990.

[2] R. Brinkmann and R. Dreschler. RTL-Datapath Verification using Integer Linear Programming . In Proc. of 15th VLSI Design Conf., pages 741-746, Jan. 2001.

[3] G. Dantzig and B. Eaves. Fourier-Motzkin Elimination and its Dual. Journal of Combinatorial Theory, A(14):288-297, 1973.

[4] M. Davis, G. Logemann, and D. Loveland. A Machine Program for Theorem Proving. Comm. of the ACM, 5(7):394-297, 1962.

[5] J.-C. Filliâtre, S. Owre, H. Rueß, and N. Shankar. ICS: Integrated Canonization and Solving. Computer-Aided Verification, CAV'2001, pages 246-249, 2001.

[6] I. Ghosh and M. Fujita. Automatic Test Pattern Generation for Functional Register-Transfer Level Circuits using Assignment Decision Diagrams. IEEE Transactions on CAD., 20(3):402-415, march 2001.

[7] S. Graf and H. Saidi. Construction of Abstract State Graphs with PVS, Computer-Aided Verification, CAV '97, pages 72-83, 1997.

[8] T. Hickey, Q. Ju, and M. H. V. Emden. Interval Arithmetic: Principles to Implementation. Journal of the ACM, 48(5):1038-1068, 2001.

[9] M. K. Iyer, G. Parthasarathy, and K.-T. Cheng. Efficient Conflict Based Learning in a Constraint Solver for RTL Circuits. in Proceedings of DATE'2004). pages 666-671, Mar 2005.

[10] W. Kunz and D. Pradhan. Recursive Learning: A New Implication Technique for Efficient Solutions to CAD Problems. IEEE Trans. on CAD, 13:1143-1158, Sept. 1994.

[11] J.P Marques-Silva and K.A. Sakallah. GRASP - A Search Algorithm for Propositional Satisfiability. IEEE Trans. on Computers, 48(5):506-521, 1999.

[12] G. Parthasarathy, M.K. Iyer, K.-T. Cheng, and Li.C. Wang. An Efficient Finite-domain Constraint Solver for RTL Circuits. In 41st DAC, June 2004.

[13] W. Kelly, et al., The Omega Calculator and Library v1.1.0. Technical report, Dept. of CS, UMCP, November 1996.

[14] R.E. Moore. Interval Analysis. Prentice-Hall, NJ, 1966.

[15] S. Seshia, S. Lahiri, and R. Bryant. A Hybrid SAT-based Decision Procedure for Separation Logic with Uninterpreted Functions. In 40th DAC, pages 425-430, June 2003.

[16] A. Stump, C. W. Barrett, and D. L. Dill. CVC: a Cooperating Validity Checker. In Computer-Aided Verification, CAV'2002, pages 500-504, July 2002.

[17] J.-K. Zhao, E. Rudnick, and J. Patel. Static Logic Implication with Application to Redundancy Identification. In Proc. of the 15th VLSI Test Symp., pages 288-293, April 1997. 


\title{
Normalization at the Arithmetic Bit Level
}

Markus Wedler, Dominik Stoffel, Wolfgang Kunz

Dept. of Electrical \& Computer Eng., University of Kaiserslautern/Germany

\begin{abstract}
We propose a normalization technique for verifying arithmetic circuits in a bounded model checking environment. Our technique operates on the arithmetic bit level $(A B L)$ description of the arithmetic circuit parts and the property. The ABL description can easily be provided by the front-end of an RTL property checker. The proposed normalization greatly simplifies the SAT instances to be solved for arithmetic circuit verification. Our approach has been applied successfully to verify the integer pipeline of an industrial microprocessor with advanced DSP capabilities.
\end{abstract}

Keywords: Property checking, arithmetic bit level normalization, SAT

\section{REFERENCES}

[1] Infineon TriCore 2 Architecural Manual. http://www.infineon.com/tricore.

[2] G. Audemard, P. Bertoli, A. Cimatti, A. Kornilowicz, and R. Sebastiani. A SAT-based approach for solving formulas over boolean and linear mathematical propositions. In Proc. Conference on Automated Deduction (CADE), pages 195-210, 2002.

[3] A. Biere, A. Cimatti, E. M. Clarke, M. Fujita, and Y. Zhu. Symbolic model checking using SAT procedures instead of BDDs. In Proc. Intl. Design Automation Conference (DAC-99), pages 317-320, June 1999.

[4] R. Brinkmann and R. Drechsler. RTL-datapath verification using integer linear programming. In Proc. Asia and South Pacific Design Automation Conference (ASPDAC-02), Bangalore, India, 2002.

[5] D. Chai and A. Kuehlmann. A fast pseudo-boolean constraint solver. In Proc. Design Automation Conference (DAC-03), pages 830-835, 2003.

[6] N. Een and N. S" orensson. An extensible SAT-solver. In Proc. 6. Intl. Conf. on Theory and Applications of Satisfiability Testing(SAT 2003), May 2003.

[7] F. Fallah, S. Devadas, and K. Keutzer. Functional vector generation for HDL models using linear programming and boolean satisfiability. IEEE Transactions on CAD, CAD-20(8), 2001.

[8] P. Johannsen. BOOSTER: Speeding up RTL property checking of digital designs by word-level abstraction. In Proc.Intl. Conf. Computer Aided Verification(CAV-01), pages 373-377, July 2001.

[9] P. Johannsen and R. Drechsler. Formal verification on the RT level computing one-to-one design abstractions by signal width reduction. In Proc. IFIP International Conference on Very Large Scale Integration(IFIP VLSI-SOC 2001), Montpellier, France, 2001.

[10] D. Stoffel and W. Kunz. Verification of integer multipliers on the arithmetic bit level. In Proc. International Conference on Computer-Aided Design (ICCAD-01), pages 183-189, San Jose, CA, November 2001.

[11] M. Wedler, D. Stoffel, and W. Kunz. Arithemetik reasoning in DPLL-based SAT solving. In Proc. Conference on Design, Automation and Test in Europe (DATE-04), Paris, France, 2004.

[12] K. Winkelmann, D. Stoffel, G. Fey, and H. Trylus. Cost-efficient block verification for a UMTS up-link chiprate coprocessor. In Proc. Conference on Design, Automation and Test in Europe (DATE-04), Paris, France, 2004.

[13] Z. Zeng, M. Ciesielski, and B. Rouzeyre. Functional test generation using constraint logic programming. In Proc. IFIP International Conference on Very Large Scale Integration (IFIP VLSI-SOC 2001), Montpellier, France, 2001.

[14] Z. Zeng, P. Kalla, and M. Ciesielski. LPSAT: A unified approach to RTL satisfiability. In Proc. Conference on Design, Automation and Test in Europe (DATE-01), Munich, Germany, 2001. 


\title{
Exploiting Suspected Redundancy without Proving It
}

\author{
Hari Mony ${ }^{1}$, Jason Baumgartner ${ }^{1}$, Viresh Paruthi ${ }^{1}$, Robert Kanzelman ${ }^{2}$ \\ ${ }^{1}$ IBM Systems Group, Austin, TX \\ ${ }^{2}$ IBM Engineering \& Technology Services, Rochester, MN
}

\begin{abstract}
We present several improvements to general-purpose sequential redundancy removal. (1) We propose using a robust variety of synergistic transformation and verification algorithms to process the individual proof obligations. This enables greater speed and scalability, and identifies a significantly greater degree of redundancy, than prior approaches. (2) We generalize upon traditional redundancy removal and utilize the speculatively-reduced model to enhance bounded search, without needing to complete any proofs.

Keywords: sequential redundancy removal, sequential equivalence checking, correctnesspreserving transformations

\section{REFERENCES}

[1] A. Kuehlmann, V. Paruthi, F. Krohm, and M. Ganai, "Robust Boolean reasoning for equivalence checking and functional property verification," TCAD, Dec. 2002.

[2] S.-Y. Huang, K.-T. Cheng, K.-C. Chen, C.-Y. Huang, and F. Brewer, "AQUILA: An equivalence checking system for large sequential designs," IEEE Trans. Computers, May 2000.

[3] C. A. J. van Eijk, "Sequential equivalence checking without state space traversal," in DATE, Mar. 1998.

[4] P. Bjesse and K. Claessen, "SAT-based verification without state space traversal," in FMCAD, Nov. 2000.

[5] K. Ng, M. R. Prasad, R. Mukherjee, and J. Jain, "Solving the latch mapping problem in an industrial setting," in $D A C, 2003$.

[6] H. Mony, J. Baumgartner, V. Paruthi, R. Kanzelman, and A. Kuehlmann, "Scalable automated verification via expert-system guided transformations," in FMCAD, Nov. 2004.

[7] A. Kuehlmann and J. Baumgartner, "Transformation-based verification using generalized retiming," in $C A V$, July 2001.

[8] M. Mneimneh and K. Sakallah, "REVERSE: Efficient sequential verification for retiming," in IWLS, May 2003.

[9] S.-Y. Huang, K.-T. Cheng, and K.-C. Chen, "On verifying the correctness of retimed circuits," in Symp. on VLSI, Mar. 1996.

[10] J. Baumgartner and A. Kuehlmann, "Min-area retiming on flexible circuit structures," in ICCAD, Nov. 2001.

[11] D. Anastasakis, L. Mcllwain, and S. Pilarski, "Efficient equivalence checking with partitions and hierarchical cut-points," in $D A C$, June 2004.

[12] D. Wang, SAT based Abstraction Refinement for Hardware Verification. PhD thesis, Carnegie Mellon University, 2003.

[13] I.-H. Moon, H. H. Kwak, J. Kukula, T. Shiple, and C. Pixley, "Simplifying circuits for formal verification using parametric representation," in FMCAD, Nov. 2002.
\end{abstract}


DAC05, pages 467-470

\title{
Multi-threaded Reachability
}

Debashis Sahoo ${ }^{1}$, Jawahar Jain ${ }^{2}$, Subramanian K. Iyer ${ }^{3}$, David L. Dill', E. Allen Emerson ${ }^{3}$

${ }^{1}$ Stanford University

${ }^{2}$ Fujitsu Labs of America, Inc.

${ }^{3}$ University of Texas at Austin

\begin{abstract}
Partitioned BDD-based algorithms have been proposed in the literature to solve the memory explosion problem in BDD-based verification. Such algorithms can be at times ineffective as they suffer from the problem of scheduling the relative order in which the partitions are processed. In this paper we present a novel multi-threaded reachability algorithm that avoids this scheduling problem while increasing the latent parallelism in partitioned state space traversal. We show that in most cases our method is significantly faster than both the standard reachability algorithm as well as the existing partitioned approaches. The gains are further magnified when our threaded implementation is evaluated in the context of a parallel framework.
\end{abstract}

Keywords: Reachability Analysis, Parallel, Multi-threaded

\section{REFERENCES}

[1] R. Bryant. Graph-based Algorithms for Boolean Function Manipulation. IEEE Trans. Comput., C-35:677-691, 1986.

[2] G. Cabodi, P. Camurati, L. Lavagno, and S. Quer. Disjunctive partitioning and partial iterative squaring: An effective approach for symbolic traversal of large circuits. In $D A C$, pages 728-733, 1997.

[3] O. Coudert, C. Berthet, and J. C. Madre. Verification of sequential machines based on symbolic execution. In Proc. Of the Workshop on Automatic Verification Methods for Finite State Systems, 1989.

[4] H. Garavel, R. Mateescu, and I. Smarandache. Parallel state space construction for model-checking. In SPIN workshop on Model checking of software, pages 217-234. Springer-Verlag New York, Inc., 2001.

[5] T. Heyman, D. Geist, O. Grumberg, and A. Schuster. Achieving scalability in parallel reachability analysis of very large circuits. In $C A V, 2000$.

[6] S. Iyer, D. Sahoo, C. Stangier, A. Narayan, and J. Jain. Improved symbolic Verification Using Partitioning Techniques. In Proc. of CHARME 2003, volume 2860 of Lecture Notes in Computer Science, 2003.

[7] J. Jain. et. al., Functional Partitioning for Verification and Related Problems. Brown/MIT VLSI Conference, 1992.

[8] K. L. McMillan. Symbolic Model Checking. Kluwer Academic Publishers, 1993.

[9] A. Narayan. et. al., Reachability Analysis Using Partitioned-ROBDDs. In ICCAD, pages 388-393, 1997.

[10] C. Pixley and J. Havlicek. A verification synergy: Constraint-based verification. In Electronic Design Processes, 2003.

[11] D. Sahoo and S. Iyer. et. al., A Partitioning Methodology for BDD-based Verification. In FMCAD, 2004.

[12] F. Somenzi. CUDD: CU Decision Diagram Package ftp://vlsi.colorado.edu/pub, 2001.

[13] U. Stern and D. L. Dill. Parallelizing the murphy verifier. In $C A V, 1997$.

[14] T. Stornetta and F. Brewer. Implementation of an efficient parallel BDD package. In DAC, pages 641-644, 1996.

[15] VIS. Verilog Benchmarks http://vlsi.colorado.edu/ vis/.

[16] B. Yang and D. R. O'Hallaron. Parallel breadth-first bdd construction. In symposium on Principles and practice of parallel programming, pages 145-156. ACM Press, 1997. 


\title{
Automatic Generation of Customized Discrete Fourier Transform IPs
}

Grace Nordin, Peter A. Milder, James C. Hoe, and Markus Püschel

Electrical and Computer Engineering Department, Carnegie Mellon University, Pittsburgh, PA, U.S.A.

\begin{abstract}
This paper presents a parameterized soft core generator for the discrete Fourier transform (DFT). Reusable IPs of digital signal processing (DSP) kernels are important time-saving resources in DSP hardware development. Unfortunately, reusable IPs, however optimized, can introduce inefficiencies because they cannot fit the exact requirements of every application context. Given the well-understood and regular computation in DSP kernels, an automatic tool can generate high-quality ready-to-use IPs customized to user-specified cost/performance tradeoffs (beyond basic parameters such as input size and data format). The paper shows that the generated DFT cores can match closely the performance and cost of DFT cores from the Xilinx LogiCore library. Furthermore, the generator can yield DFT cores over a range of different performance/cost tradeoff points that are not available from the library.

Keywords: Discrete Fourier transform, IP, design generator, FPGA

\section{REFERENCES}

[1] S. Choi, R. Scrofano, V. K. Prasanna, and J.-W. Jang. Energy-efficient signal processing using FPGAs. In Proc. International Symposium on Field Programmable Gate Arrays, 2003.

[2] P. Kumhom, J. Johnson, and P. Nagvajara. Design, optimization, and implementation of a universal FFT processor. In Proc. 13th IEEE ASIC/SOC Conference, 2000.

[3] Spiral project. www.spiral.net.

[4] J. Takala, T. Jarvinen, P. Salmela, and D. Akopian. Multi-port interconnection networks for radix-r algorithms. In Proc. IEEE Intl. Conf. Acoustics, Speech, Signal Processing, 2001.

[5] C. Van Loan. Computational Framework of the Fast Fourier Transform. SIAM, 1992.

[6] Xilinx, Inc. Xilinx LogiCore: Fast Fourier Transform v3.1, November 2004.
\end{abstract}




\title{
Race-Condition-Aware Clock Skew Scheduling
}

Shih-Hsu Huang, Yow-Tyng Nieh, Feng-Pin Lu

Department of Electronic Engineering, Chung Yuan Christian University, Chung Li, Taiwan, R.O.C.

\begin{abstract}
The race conditions often limit the smallest feasible clock period that the optimal clock skew scheduling can achieve. Therefore, the combination of clock skew scheduling and delay insertion (for resolving the race conditions) may lead to further clock period reduction. However, the interactions between clock skew scheduling and delay insertion have not been well studied. In this paper, we provide a fresh viewpoint to look at this problem. A novel approach, called racecondition-aware (RCA) clock skew scheduling, is proposed to determine the clock skew schedule by taking the race conditions into account. Our objective is not only to optimize the clock period, but also to heuristically minimize the required inserted delay. Compared with previous work, our approach has significant improvement in the time complexity.
\end{abstract}

Keywords: High performance, Sequential circuits, Timing optimization

\section{REFERENCES}

[1] J.P. Fishburn, “Clock Skew Optimization”, IEEE Trans. On Computers, Vol. 39, No. 7, pp. 945-951, 1990.

[2] R.B. Deokar and S.S. Sapatnekar, “A Graph-Theoretic Approach to Clock Skew Optimization”, Proc. of IEEE International Symposium on Circuits and Systems, Vol. 1, pp.407-410, 1994.

[3] M.C. Papaefthymiou, "Understanding Retiming through Maximum Average-Delay Cycles", Mathematical Systems Theory, Vol. 27, pp. 65-84, 1994.

[4] N.V. Shenoy, R.K. Brayton and A.L. Sangiovanni-Vincentelli, "Minimum Padding to Satisfy Short Path Constraints", Proc. of IEEE/ACM International Conference on Computer Aided Design, pp. 156-161, 1993.

[5] S.H. Huang and Y.T. Nieh, "Clock Period Minimization of Non-Zero Clock Skew Circuits", Proc. of IEEE/ACM International Conference on Computer Aided Design, pp. 809—812, 2003. 


\title{
A Novel Synthesis Approach for Active Leakage Power Reduction Using Dynamic Supply Gating
}

Swarup Bhunia, Nilanjan Banerjee, Qikai Chen, Hamid Mahmoodi, and Kaushik Roy School of Electrical and Computer Engineering, Purdue University, West Lafayette.

\begin{abstract}
:
Due to exponential increase in subthreshold leakage with technology scaling and temperature increase, leakage power is becoming a major fraction of total power in the active mode. We present a novel low-cost design methodology with associated synthesis flow for reducing both switching and active leakage power using dynamic supply gating. A logic synthesis approach based on Shannon expansion is proposed that dynamically applies supply gating to idle parts of general logic circuits even when they are performing useful computation. Experimental results on a set of MCNC benchmark circuits in a predictive 70nm process exhibits improvements of $15 \%$ to $88 \%$ in total active power compared to the results obtained by a conventional optimization flow.
\end{abstract}

General Terms: Algorithms, Design, Performance

\section{References}

[1] S. Mukhopadhyay et al., "Modeling and estimation of failure probability due to parameter variations in nanoscale SRAMs for yield enhancement," Symp. on VLSI Circuits, pp. 12-14, 2003.

[2] Y. Taur and T. H. Ning, Fundamentals of Modern VLSI Devices, New York: Cambridge Univ. Press, 1998

[3] G. Sery et al., "Life is CMOS: why chase the life after?" Design Automation Conf., 2002, pp. 78-83.

[4] R. Krishnarnurthy et al., "High-performance and low-power challenges for sub-70 nm microprocessor circuits," CICC, pp. 12-15, May 2002.

[5] J.W. Tschanz et al. "Dynamic sleep transistor and body bias for active leakage power control of microprocessors," IEEE JSSC, vol. 38, pp. 1838-1845, 2003.

[6] A. Chandrakasan, Design of High-Performance Microprocessor Circuits, IEEE Press.

[7] A. Keshavarzi et al., "Effectiveness of reverse body bias for leakage control in scaled dual Vt CMOS ICs," ISLPED, pp. 207-212, 2001.

[8] L. Lavagno et al., Design Automation Conf. pages 254-260, 1995.

[9] M. Liu et al., "Leakage power reduction by dual-Vth designs under probabilistic analysis of Vth variation," I SLPED, pp. 2-7, Aug. 2004.

[10] Predictive Technology Model,www.device.eecs.berkeley.edu

[11] SIS, University of California at Berkeley. 


\title{
Designing Logic Circuits for Probabilistic Computation in the Presence of Noise
}

\author{
K. Nepal, R. I. Bahar, J. Mundy, W. R. Patterson, and A. Zaslavsky \\ Brown University, Division of Engineering, Providence, RI
}

\begin{abstract}
As Si CMOS devices are scaled down into the nanoscale regime, current computer architecture approaches are reaching their practical limits. Future nano-architectures will confront devices and interconnections with a large number of inherent defects, which motivates the search for new architectural paradigms. In this paper, we examine probabilistic-based design methodologies for nanoscale computer architectures based onMarkov random fields (MRF). The MRF approach can express arbitrary logic circuits and the logic operation is achieved by maximizing the probability of correct state configurations in the logic network depending on the interaction of neighboring circuit nodes. The computation proceeds via probabilistic propagation of states through the circuit. Crucially, the MRF logic can be implemented in modified CMOS-based circuitry that trades off circuit area and operation speed for the crucial fault tolerance and noise immunity. This paper builds on the recent demonstration that significant immunity to faulty individual devices or dynamically occurring signal errors can be achieved by the propagation of state probabilities over an MRF network. In particular, we are interested in CMOS-based circuits that work reliably at very low supply voltages $\left(V_{D D}=0.1-0.2 \mathrm{~V}\right)$, where standard CMOS would fail due to thermal and crosstalk noise, and transistor threshold variation. In this paper, we present results for simulated probabilistic test circuits for elementary logic components and well as small circuits taken from the MCNC91 benchmark suite and we show greatly improved noise immunity operating at very low $V_{D D}$. The MRF framework extends to all levels of a design, where formally optimum probabilistic computation can be implemented as a natural element of the processing structure.
\end{abstract}

Keywords: noise immunity, reliability, subthreshold operation, probabilistic computing, Markov random fields, nanodevices

\section{REFERENCES}

[1] G. K. Celler and S. Cristoloveanu, "Frontiers of silicon-on-insulator", J. Appl. Phys. 93, 4955 (2003).

[2] S. Luryi, J. M. Xu, and A. Zaslavsky, eds., Future Trends in Microelectronics: The Nano, the Giga, and the Ultra, New York: Wiley, 2004.

[3] H. S. P. Wong, "Beyond the conventional transistor", IBM J. Res. Dev. 46, 133 (2002).

[4] The latest publicly released version of the ITRS roadmap is available on the http://public.itrs.net web site.

[5] B. Doris et al, "Extreme scaling with ultra-thin Si channel MOSFETs", Tech. Digest IEDM (2002), p. 267.

[6] H. Iwai, "The future of CMOS downscaling", in: S. Luryi, J. M. Xu, and A. Zaslavsky, eds., Future Trends in Microelectronics: The Nano, the Giga, and the Ultra, New York: Wiley, 2004, pp. 23-33.

[7] R. Chellappa, Markov Random Fields: Theory and Applications, New York: Academic Press, 1993

[8] S. Z. Li, Markov Random Field Modeling in Computer Vision, Berlin: Springer-Verlag, 1995.

[9] R. I. Bahar, J. Mundy, and J. Chen, "A Probabilistic-based Design Methodology for Nanoscale Computation”, International Conference on CAD, Nov. 2003.

[10] J. Besag, "Spatial interaction and the statistical analysis of lattice systems", J. Royal Statistical Soc. Ser. B 36, 192 (1994).

[11] Available at http://www-device.eecs.berkeley.edu/ ptm/ .

[12] V. M. Polyakov and F. Schwierz, "Excessive noise in nanoscaled double-gate MOSFETs: A Monte Carlo study", Semicond. Sci. Technol. 19, 145 (2004).

[13] S. Narendra, V. De, S. Borkar, D. A. Antoniadis, and A. P. Chandrakasan, "Full-chip subthreshold leakage power prediction and reduction techniques for sub-0.18 $\mu \mathrm{m}$ CMOS", IEEE J. Solid-State Circuits 39, 501 (2004).

[14] R. Sarpeshkar, T. Delbrueck, C. A. Mead, "White Noise in MOS Transistors and Resistors," IEEE Circuits and Devices Magazine, 623 (Nov. 1993). 
[15] E. Suzuki, K. Ishii, S. Kanemaru, T. Maeda, T. Tsutsumi, T. Sekigawa, K. Nagai, and H. Hiroshima, "Highly suppressed short-channel effects in ultrathin SOI n-MOSFETs" IEEE Trans. Electron Dev. 47, 354 (2000).

[16] T. Ernst, S. Cristoloveanu, G. Ghibaudo, T. Ouisse, S. Horiguchi, Y. Ono, Y. Takahashi, and K. Murase, "Ultimately thin double-gate SOI MOSFETs", IEEE Trans. Electron Dev. 50, 830 (2003).

[17] S. Kullback, Information Theory and Statistics, New York: Dover, 1969.

[18] E. M. Sentovich et al., "Sequential circuit design using synthesis and optimization", International Conference on Computer Design, Oct. 1992.

[19] A. J. Viterbi, "Error bounds for convolutional codes and an asymptotically optimum decoding algorithm", IEEE Trans. Information Theory 13, 260 (1967).

[20] S. Geman and K. Kochanek, "Dynamic programming and the graphical representation of error-correcting codes", IEEE Trans. Information Theory 47, 549 (2001).

[21] S. Melnikoff, S. Quigley, and M. Russell, "Implementing a hidden Markov model speech recognition system in programmable logic", 11th Intern. Workshop Field-Programmable Logic Applications, Aug. 2001. 


\title{
A Lattice-Based Framework for the Classification and Design of Asynchronous Pipelines
}

\author{
Peggy B. McGee, Steven M. Nowick \\ Department of Computer Science, Columbia University, New York, NY 10027
}

\begin{abstract}
This paper presents a unifying framework for the modeling of asynchronous pipeline circuits. A pipeline protocol is captured in a graph-based model which defines the partial ordering of both its control and data events. The relationship between an entire space of different protocols is then captured in a semi-lattice, which has well-defined top and bottom elements, corresponding to the most concurrent and least concurrent protocol variants, respectively. This framework also provides a set of correct-by-construction transformation rules which allows for the systematic exploration of the entire design space by their successive application. To the best of our knowledge, this is the first formal framework for asynchronous pipelines which can capture protocols from a variety of logic style families, including both dynamic and static. It is also the first to provide a formal foundation for the design-space exploration of asynchronous pipelines.

Keywords: pipeline, framework, asynchronous, digital design, protocols
\end{abstract}

\section{REFERENCES}

[1] P. Beerel and A. Lines. Personal communication.

[2] I. Blunno, J. Cortadella, A. Kondratyev, L. Lavagno, K. Lwin, and C. Sotiriou. Handshake protocols for desynchronization. In Proceedings of the $10^{\text {th }}$ International Symposium on Asynchronous Circuits and Systems, 2004.

[3] B. Davey and H. Priestley. Introduction to Lattices and Order. Cambridge University Press, 2002.

[4] S. B. Furber and P. Day. Four-phase micropipeline latch control circuits. IEEE Transactions on Very Large Scale Integration (VLSI) Systems, 4(2), June 1996.

[5] A. Kondratyev, J. Cortadella, M. Kishinevsky, L. Lavagno, and A. Yakovlev. Automatic synthesis and optimization of partially specified asynchronous systems. In Proceedings, 36th Design Automation Conference, 1999.

[6] A. Lines. Pipelined asynchronous circuits. Master's thesis, California Institute of Technology, June 1996, revised 1998.

[7] R. Manohar, T.-K. Lee, and A. J. Martin. Projection: A synthesis technique for concurrent systems. In Proceedings of 5th International Symposium on Asynchronous Circuits and Systems, Apr. 1999.

[8] A. J. Martin, A. Lines, R. Manohar, M. Nystroem, P. Penzes, R. Southworth, and U. Cummings. The design of an asynchronous MIPS R3000 microprocessor. In Proceedings of Conference on Advanced Research in VLSI, 1997.

[9] P. McGee and S. Nowick. A unifying lattice-based framework for the classification and design of asynchronous pipelines. ACM/IEEE International Workshop on Timing Issues, 2005.

[10] R. Ozdag and P. Beerel. High-speed QDI asynchronous pipelines. In Proceedings of 8th International Symposium on Asynchronous Circuits and Systems, 2002.

[11] C. Seitz. .System Timing,. Chapter 7 in Introduction to VLSI Systems, eds. C. Mead and L. Conway. AddisionWesley, 1980.

[12] M. Singh and S. M. Nowick. Fine-grain pipelined asynchronous adders for high-speed DSP applications. In IEEE Computer Society Annual Workshop on VLSI, 2000.

[13] M. Singh and S. M. Nowick. Mousetrap: Ultra-high-speed transition-signaling asynchronous pipelines. In International Conf. Computer Design (ICCD), 2001.

[14] I. Sutherland. Micropipelines. In Communications of the ACM, 1989.

[15] T. Williams. Self-Timed Rings and Their Applications to Division. PhD thesis, Stanford University, Jun 1991.

[16] A. Yakovlev, A. Koelmans, and L. Lavagno. High-level modeling and design of asynchronous interface logic. IEEE Design and Test of Computers, 12, 1995. 


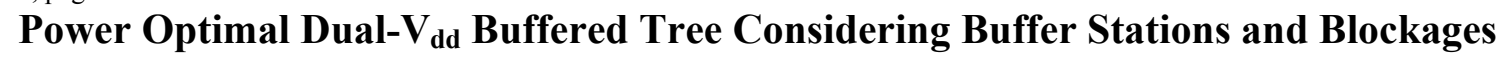

\author{
King Ho Tam and Lei He \\ Electrical Engineering Dept., Univ. of California, Los Angeles, CA, USA
}

\begin{abstract}
This paper presents the first in-depth study on applying dual $V_{\mathbf{d d}}$ buffers to buffer insertion and multi-sink buffered tree construction for power minimization under delay constraint. To tackle the problem of dramatic complexity increment due to simultaneous delay and power consideration and increased buffer choices, we develop a sampling-based sub-solutions (i.e. options) propagation method and a balanced search tree-based data structure for option pruning. We obtain 17x speedup with little loss of optimality compared to the exact option propagation. Moreover, compared to buffer insertion with single $V_{\text {dd }}$ buffers, dual- $V_{\text {dd }}$ buffers reduce power by $23 \%$ at the minimum delay specification. In addition, compared to the delay-optimal tree using single $V_{\text {dd }}$ buffers, our power-optimal buffered tree reduces power by $7 \%$ and $18 \%$ at the minimum delay specification when single $V_{\text {dd }}$ and dual $V_{\text {dd }}$ buffers are used respectively.
\end{abstract}

Keywords: Low power, buffer insertion, detail routing

\title{
REFERENCES
}

[1] J. Lillis, C. Cheng, and T. Lin, "Optimal wire sizing and buffer insertion for low power and a generalized delay model," in ICCAD, Nov. 1995.

[2] T. Okamoto and J. Cong, "Buffered Steiner tree construction with wire sizing for interconnect layout optimization," in ICCAD, Nov. 1996.

[3] J. Lillis, C. Cheng, and T. Lin, "Simultaneous routing and buffer insertion for high performance interconnect," in GLVLSI Symp., 1996.

[4] C. Alpert, G. Gandham, J. Hu, J. Neves, S. Quay, and S. Sapatnekar, "Steiner tree optimization for buffers, blockages and bays," in ISCAS, May 2001.

[5] J. Hu, C. Alpert, S. Quay, and G. Gandham, "Buffer insertion with adaptive blockage avoidance," TCAD, vol. 22, no. 4, pp. 492-498, 2003.

[6] J. Cong and X. Yuan, "Routing tree construction under fixed buffer locations," in $D A C$, Jun 2000.

[7] W. Chen, M. Pedram, and P. Buch, "Buffered routing tree construction under buffer placement blockages," in $A S P-D A C$, Jan 2002.

[8] F. Li, Y. Lin, and L. He, "Vdd programmability to reduce fpga interconnect power," in ICCAD, Nov 2004.

[9] K. H. Tam and L. He, "Power optimal dual-vdd buffered tree considering buffer stations and blockages," in University of California, Los Angeles, Technical Report, UCLA Engr 05-259, 2005.

[10] H. Bakoglu, Circuits, Interconnects and Packaging for VLSI. Addison-Wesley, 1990.

[11] K. Banerjee and A. Mehrotra, "A power-optimal repeater insertion methodology for global interconnects in nanometer designs," TCAD, vol. 49, no. 11, pp. 2001-2007, 2002.

[12] C. Alpert, D. Devgan, and C. Kashyap, "RC delay metrics for performance optimization," TCAD, vol. 20, no. 5, pp. 571-582, 2001.

[13] "Berkeley predictive technology model," in http://www-device.eecs.berkeley.edu/ ptm.

[14] D. Warme, P. Winter, and M. Zachariasen, “Geosteiner," in http://www.diku.dk/geosteiner, 2003.

[15] Y. Lin and L. He, "Leakage efficient chip-level dual-vdd assignment with time slack allocation for fpga power reduction," in $D A C$, Jun 2005. 


\title{
Net Weighting to Reduce Repeater Counts during Placement
}

\author{
Brent Goplen*, Prashant Saxena**, Sachin Sapatnekar* \\ *Department of ECE, University of Minnesota, Minneapolis, MN \\ **Advanced Technology Group, Synopsys Inc., Hillsboro, OR
}

\begin{abstract}
We demonstrate how to use placement to ameliorate the predicted repeater explosion problem caused by poor interconnect scaling. We achieve repeater count reduction by dynamically modifying net weights in a context-sensitive manner during global placement and coarse legalization. Our scheme, which models layer assignment as well as valid inter-repeater distance ranges, can decrease the repeater counts significantly with minimal impact on wirelength.

Keywords: Placement, Net weighting, Force-directed placement, Repeater, Buffering, Scaling, Interconnect

\section{REFERENCES}

[1] Bakoglu, H. B. Circuits, Interconnects and Packaging for VLSI. Addison-Wesley: Reading MA, 1990.

[2] Saxena, P., Menezes, N., Cocchini, P., and Kirkpatrick, D. A. Repeater Scaling and its Impact on CAD. TCAD, 2004, 23(4), 451-463.

[3] Cong, J., Kong T., and Pan, D. Z. Buffer Block Planning for Interconnect-Driven Floorplanning. ICCAD '99, 358-363.

[4] Tsay, R. S. and Koehl, J. An Analytic Net Weighting Approach for Performance Optimization in Circuit Placement. $D A C$ '91, 620-625.

[5] Ren, H., Pan, D. Z., and Kung, D. S. Sensitivity Guided Net Weighting for Placement Driven Synthesis. ISPD '04, 10-17.

[6] Rajagopal, K., Shaked, T., Parasuram, Y., Cao, T., Chowdhary, A., and Halpin, B. Timing Driven Force Directed Placement with Physical Net Constraints. ISPD ‘03, 60-66.

[7] Obermeier, B. and Johannes, F. M. Temperature-Aware Global Placement. ASP-DAC ‘04, 143-148.

[8] Vaishnav, H. and Pedram, M. PCUBE: A Performance Driven Placement Algorithm for Low-Power Design. Euro-DAC'93, 72-77.

[9] Cheon, Y., Ho, P.-H., Kahng, A. B., Reda, S. and Wang, Q. Power-Aware Placement. $D A C$ ‘ 05, to appear.

[10] Eisenmann, H., and Johannes, F. M. Generic Global Placement and Floorplanning. DAC '98, 269-274.

[11] Saxena, P., and Halpin, B. Modeling Repeaters Explicitly Within Analytical Placement. DAC '04, 699-704.

[12] Hur, S.-W., and Lillis, J. Mongrel: Hybrid Techniques for Standard Cell Placement. ICCAD ‘00, 165-170.

[13] Hur, S.-W., Cao, T., Rajagopal, K., Parasuram, Y., Chowdhary, A., Tiourin, V., and Halpin, B. Force Directed Mongrel with Physical Net Constraints. DAC '03, 214-219.

[14] Sigl, G., Doll, K., and Johannes, F. M. Analytical Placement: A Linear or a Quadratic Objective Function? $D A C$ '91, 427-432.

[15] Akkiraju, N., and Mohan, M. Spec-based Flip-flop and Buffer Insertion. ICCAD '03, 270-275.

[16] Alpert, C. J., Hu, J., Sapatnekar, S., and Villarubia, P. Practical Methodology for Early Buffer and Wire Resource Allocation, $D A C$ ‘01, 189-194.
\end{abstract}




\author{
Path Based Buffer Insertion \\ C. N. Sze, Charles J. Alpert†, Jiang Hu and Weiping Shi \\ EE Dept., Texas A\&M Univ., College Station, TX \\ $\uparrow$ IBM Corp., Austin, TX, USA
}

\begin{abstract}
Along with the progress of VLSI technology, buffer insertion plays an increasingly critical role on affecting circuit design and performance. Traditional buffer insertion algorithms are mostly net based and therefore often result in sub-optimal delay or unnecessary buffer expense due to the lack of global view. In this paper, we propose a novel path based buffer insertion scheme which can overcome the weakness of the net based approaches. We also discuss some potential difficulties of the path based buffer insertion approach and propose solutions to them. A fast estimation on buffered delay is employed to improve the solution quality. Gate sizing is also considered at the same time. Experimental results show that our method can efficiently reduce buffer/gate cost significantly (by $71 \%$ on average) when compared to traditional net based approaches. To the best of our knowledge, this is the first work on path based buffer insertion
\end{abstract} and simultaneous gate sizing.

Keywords: Buffer Insertion, Interconnect Synthesis, PowerMinimization, Global Routing, Layout, Physical Design

\title{
REFERENCES
}

[1] C. J. Alpert, C. Chu, G. Gandham, M. Hrkic, J. Hu, C. Kashyap, and S. T. Quay. Simultaneous driver sizing and buffer insertion using delay penalty estimation technique. IEEE Trans. on CAD, 23(1):136-141, January 2004.

[2] C. J. Alpert and A. Devgan. Wire segmenting for improved buffer insertion. In Proc. of DAC, pages 588-593, 1997.

[3] C. J. Alpert, A. Devgan, and S. T. Quay. Buffer insertion for noise and delay optimization. In Proc. of DAC, pages 362-367, 1998.

[4] C. J. Alpert, A. Devgan, and S. T. Quay. Buffer insertion with accurate gate and interconnect delay computation. In Proc. of DAC, pages 479-484, 1999.

[5] C. J. Alpert, M. Hrkic, J. Hu, and S. T. Quay. Fast and flexible buffer trees that navigate the physical layout environment. In Proc. of DAC, pages 24-29, 2004.

[6] C. J. Alpert, J. Hu, S. S. Sapatnekar, and C. N. Sze. Accurate Estimation of Global Buffer Delay within a Floorplan. In Proc. Of ICCAD, pages 706-711, 2004.

[7] H. B. Bakoglu. Circuits, interconnections and packaging for VLSI. Addison-Wesley, Reading, MA, 1990.

[8] C. C. N. Chu and D. F. Wong. A quadratic programming approach to simultaneous buffer insertion/sizing and wire sizing. IEEE Trans. On CAD, 18(6):787-798, June 1999.

[9] C. C. N. Chu and D. F. Wong. Closed form solution to simultaneous buffer insertion/sizing and wire sizing. ACM Trans. on Design Automation of Electronic Systems, 6(3):343-371, July 2001.

[10] J. A. Davis, R. Venkatesan, A. Kaloyeros, M. Beylansky, S. J. Souri, K. Banerjee, K. C. Saraswat, A. Rahman, R. Reif, and J. D. Meindl. Interconnect limits on gigascale integration (GSI) in the 21st century. Proc. of IEEE, 89(3):305-324, March 2001.

[11] S. Dhar and M. A. Franklin. Optimum buffer circuits for driving long uniform lines. IEEE Journal of SolidState Circuits, 26(1):32-38, January 1991.

[12] L. P. P P. van Ginneken. Buffer placement in distributed RC-tree networks for minimal Elmore delay. In Proc. of ISCAS, pages 865-868, 1990.

[13] C. V. Kashyap, C. J. Alpert, F. Liu, and A. Devgan. Closed-form expressions for extending step delay and slew metrics to ramp inputs for RC trees IEEE Trans. on CAD, 23(4):509-516, April 2004.

[14] Y. Jiang, S. S. Sapatnekar, C. Bamji, and J. Kim. Interleaving buffer insertion and transistor sizing into a single optimization. IEEE Trans. on VLSI Systems, 6(4):625-633, December 1998.

[15] Y.-C. Ju, and R. A. Saleh. Incremental techniques for the identification of statically sensitizable critical paths. In Proc. of DAC, pages 541-546, 1991.

[16] Z. Li, C. N. Sze, C. J. Alpert, J. Hu, and W. Shi. Making fast buffer insertion even faster via approximation techniques. In Proc. Of ASPDAC, pages 13-18, 2005. 
[17] J. Lillis, Algorithms for Performance Driven Design of Integrated Circuits. PhD Thesis, UC San Diego, August 1996.

[18] J. Lillis, C. K. Cheng, and T. Y. Lin. Optimal wire sizing and buffer insertion for low power and a generalized delay model. IEEE Journal of Solid-State Circuits, 31(3):437-447, March 1996.

[19] I.-M. Liu, A. Aziz, and D. F. Wong. Meeting delay constraints in DSM by minimal repeater insertion. In Proc. of DATE, pages 436-441, 2000.

[20] I.-M. Liu, A. Aziz, D. F. Wong, and H. Zhou. An efficient buffer insertion algorithm for large networks based on Lagrangian relaxation. In Proc. of ICCD, pages 614-621, 1999.

[21] S. S. Sapatnekar. Timing. Kluwer Academic Publishers, 2004.

[22] P. Saxena, N. Menezes, P. Cocchini, and D. A. Kirkpatrick. Repeater scaling and its impact on CAD. IEEE Trans. on CAD, 23(4):451-463, April 2004.

[23] W. Shi and Z. Li. An $O(n \log n)$ time algorithm for optimal buffer insertion. In Proc. of DAC, pages 580-585, 2003. 


\title{
Diffusion-Based Placement Migration
}

\author{
Haoxing Ren ${ }^{1}$, David Z. Pan ${ }^{2}$, Charles J. Alpert ${ }^{3}$ \\ ${ }^{1}$ IBM Corp. \& Univ. of Texas at Austin, Austin, TX \\ ${ }^{2}$ ECE Department, Univ. of Texas at Austin, Austin, TX \\ ${ }^{3}$ IBM Corp., Austin, TX
}

\begin{abstract}
Placement migration is the movement of cells within an existing placement to address a variety of post-placement design closure issues, such as timing, routing congestion, signal integrity, and heat distribution. To fix a design problem, one would like to perturb the design as little as possible while preserving the integrity of the original placement. This work presents a new diffusion-based placement method based on a discrete approximation to a closed-form solution of the continuous diffusion equation. It has the advantage of smooth spreading, which helps preserve neighborhood characteristics of the original placement. Applying this technique to placement legalization demonstrates significant improvements in wire length and timing compared to other commonly used techniques.
\end{abstract}

Keywords: Placement Migration, Diffusion, Legalization

\section{REFERENCES}

[1] H. Ren, D. Z. Pan, and P. Villarrubia, "True crosstalk aware incremental placement with noise map," in Proc. Int. Conf. on Computer Aided Design, pp. 402-409, 2004.

[2] H. Eisenmann and F. M. Johannes, "Generic global placement and floorplanning," in Proc. Design Automation Conf., pp. 269-274, 1998.

[3] U. Brenner, A. Pauli, and J. Vygen, "Almost optimum placement legalization by minimum cost flow and dynamic programming," in Proc. Int. Symp. on Physical Design, pp. 2-9, 2004.

[4] A. Agnihotri, M. C. Yildiz, A. Khatkhate, A. Mathur, S. Ono, and P. H. Madden, "Fractional cut: improved recursive bisection placement," in Proc. Int. Conf. on Computer Aided Design, pp. 307-310, 2003.

[5] S. W. Hur and J. Lilis, "Mongrel: hybrid techniques for standard cell placement," in Proc. Int. Conf. on Computer Aided Design, pp. 165-170, 2000.

[6] A. B. Kahng, P. Tucker, and A. Zelikovsky, "Optimization of linear placements for wirelength minimization with free sites," in Proc. Asia and South Pacific Design Automation Conf., pp. 18-21, 1999.

[7] J. D. Plummer, M. D. Deal, and P. B. Griffin, Silicon VLSI Technology: Fundamentals, Practice, and Modeling. Prentice Hall, 2003.

[8] W. H. Press, S. A. Teukolsky, W. T. Vetterling, and B. P. Flannery, Numerical Recipes in C++. Cambridge University Press, 2002.

[9] H. Ren, D. Z. Pan, and D. Kung, "Sensitivity guided net weighting for placement driven synthesis," in Proc. Int. Symp. on Physical Design, pp. 10-17, 2004. 


\section{Is Methodology the Highway Out of Verification Hell?}

Chair: $\quad$ Gabe Moretti - EDA Consultant \& Editor, Venice, FL

Panelists: Harry Foster - Jasper Design Automation, Mountain View, CA

Janick Bergeron - Synopsys, Ottawa, Canada

Masayuki Nakamura - Sony Corporation, Shinagawa, Japan

Shrenik Mehta - Sun Microsystems, Osaki Shinagawa-Ku, Japan

Laurent Ducousso - ST Microelectronics, Grenoble, France

\section{PANEL SUMMARY}

Few would disagree that verification takes the lion's share of today's project resources. If we examine the available research, we quickly discover that verification is a significant pain point that consumes massive amounts of time and resources across a multitude of market segments. Per Gary Smith at Gartner Dataquest, verification consumes 30\% to $70 \%$ of total schedule, depending on design size. According to Collett International Research, Inc., a majority of ASICs and integrated circuits (ICs) require at least one respin with $71 \%$ of respins are due to functional bugs "verification should have caught".

With such statistics, it is easy to understand why many contend that the verification challenge is growing at a double exponential rate (that is, exponential with respect to Moore's law). Given verification's importance and its significant impact on fundamental design quality and time-tomarket demands, what is our industry doing in response? This panel explores where the methodology highway is taking us - is the destination heaven or just another level of Dante's inferno?

Respected authors and experts in verification methodology will share their insights and opinions of the two methodologies used today: verify-after-the-fact (traditional) and verify-as-you-design (emerging). For decades, simulation has necessitated a verifyafter-the-fact methodology and yet we can see from the industry research that a high percentage of silicon requires respins. With the latest advances in simulation testbenches and languages, can the verify-after-the-fact approach scale? Or, is it time for a move to a higher level of abstraction that enables a verify-as-youdesign methodology?

Industry leading chip and systems companies will discuss the methodologies they employ today to address the enormous challenge of functional verification. Questions to be addressed by our esteemed panelists include: How can we bring in schedules? What can we do to increase design quality? What cultural and organizational changes have to take place to bring quality back to the forefront of design? Where is the measurable proof of quality? What are the questions that managers should be asking themselves? What are the engines being used? What formal techniques deliver the greatest success? How important is HW/SW verification? What are the processes or methodologies being used to overcome tool or technology limitations? What is the value of assertions? How does a geographically dispersed engineering team impact design quality? What are the metrics being used to measure progress and success? And how do you know when you are done?

Today we currently don't design quality in - we TEST it in (using simulation). But, what would happen if quality was designed in from the beginning? How much could we improve the overall quality level and reduce verification time, and what would this take to do it? Finally, can migration to a new methodology be the highway out of verification hell? 
Keywords: Verification, methodology, assertions, formal verification 


\title{
Full-Chip Analysis of Leakage Power Under Process Variations, Including Spatial Correlations
}

\author{
Hongliang Chang, Sachin S. Sapatnekar \\ Dept. of Electrical and Computer Engineering, University of Minnesota
}

\begin{abstract}
In this paper, we present a method for analyzing the leakage current, and hence the leakage power, of a circuit under process parameter variations that can include spatial correlations due to intra-chip variation. A lognormal distribution is used to approximate the leakage current of each gate and the total chip leakage is determined by summing up the lognormals. In this work, Both subthreshold leakage and gate tunneling leakage are considered. The proposed method is shown to be effective in predicting the $\mathrm{CDF} / \mathrm{PDF}$ of the total chip leakage. The average errors for mean and sigma values are $-1.3 \%$ and $-4.1 \%$.
\end{abstract}

General Terms: Algorithm, Design, Performance, Reliability

\section{REFERENCES}

[1] A. A. Abu-Dayya and N. C. Beaulieu, "Comparison of methods of computing correlated lognormal sum distributions and outages for digital wireless applications," IEEE 44th Vehicular Technology Conference, vol. 1, pp. 175-179, 1994.

[2] K. A. Bowman, L. Wang, X. Tang and J. D. Meindl, “A Circuit Level Perspective of the Optimum Gate Oxide Thickness,” IEEE Transction on Electron Devices, pp. 1800-1810, 2001.

[3] H. Chang and S. S. Sapatnekar, "Statistical Timing Analysis Considering Spatial Correlations Using a Single PERT-like Traversal," International Conference on Computer Aided Design, pp. 621-625, 2003.

[4] M. Ketkar and S. S. Sapatnekar", "Standby Power Optimization via Transistor Sizing and Dual Threshold Voltage Assignment," International Conference on Computer-Aided Design, pp. 375-378, 2002.

[5] D. Lee, W. Kwong, D. Blaauw and D. Sylvester, "Analysis and Minimization Techniques for Total Leakage Considering Gate Oxide Leakage," Design Automation Conference, pp. 175-180, 2003.

[6] S. Mukhopadhyay and K. Roy, "Modeling and Estimation of Total Leakage Current in Nano-scaled CMOS Devices Considering the Effect of Parameter Variation," International Symposium on Low Power Electronics and Design, pp. 172-175, 2003.

[7] S. Narendra, V. De, S. Borkar, D. Antoniadis and A. Chandrakasan, "Full-chip sub-threshold leakage power prediction model for sub-0.18um CMOS," International Symposium on Low Power Electronics and Design, pp. 1923, 2002.

[8] S. Nassif, "Delay Variability: Sources, Impact and Trends," IEEE International Solid-State Circuits Conference, pp. 368-369, 2000.

[9] R. Rao, A. Devgan, D. Blaauw and D. Sylvester, "Parametric yield estimation considering leakage variability," Design Automation Conference, pp. 442-447, 2003

[10] R. Rao, A. Srivastava, D. Blaauw and D. Sylvester, "Statistical Estimation of Leakage Current Considering Inter- and Intra-Die Process Variation,” International Symposium on Low Power Electronics and Design, pp. 19-23, 2003.

[11] S. Sirichotiyakul, T. Edwards, C. Oh, J. Zuo, A. Dharchoudhury, R. Panda and D. Blaauw, "Stand-by power minimization through simultaneous threshold voltage selection and circuit sizing," Design Automation Conference, pp. 436-441, 1999.

[12] A. Srivastava, R. Bai, D. Blaauw and D. Sylvester, "Modeling and analysis of leakage power considering within-die process variations," International Symposium on Low Power Electronics and Design, pp. 64-67, 2002.

[13] A. Sultania, D. Sylvester, and S. S. Sapatnekar, "Tradeoffs between Gate Oxide Leakage and Delay for Dual Tox Circuits," Design Automation Conference, pp. 761 - 766, 2004.

[14] Y. Taur and T. H. Ning, Fundamentals of Modern VLSI Devices, Cambridge Univerity Press, Cambridge, UK, 1998.

[15] "Capo: A large-scale fixed-die placer from UCLA," Available at: http://vlsicad.ucsd.edu/GSRC/bookshelf/ Slots/Placement.

[16] Semiconductor Industry Association, "International Technology Roadmap for Semiconductors,"2004. Available at : http://public.itrs.net. 


\title{
Variations-Aware Low-Power Design with Voltage Scaling
}

Navid Aziził, Muhammad M. Khellah†, Vivek De†, Farid N. Najm $\neq$

$\neq$ Department of ECE, University of Toronto, Toronto, Ontario, Canada

†Circuits Research, Intel Labs, Hillsboro, Oregon

\begin{abstract}
We present a new methodology which takes into consideration the effect of Within-Die (WID) process variations on a low-voltage parallel system. We show that in the presence of process variations one should use a higher supply voltage than would otherwise be predicted to minimize the power consumption of a parallel systems. Previous analyses, which ignored WID process variations, provide a lower non-optimal supply voltage which can underestimate the energy/operation by $8.2 \mathrm{X}$. We also present a novel technique to limit the effect of temperature variations in a parallel system. As temperatures increases, the scheme reduces the power increase by $43 \%$ allowing the system to remain at it's optimal supply voltage across different temperatures.
\end{abstract}

Keywords: Process Variations, Parallel Systems, Low-Voltage

\section{REFERENCES}

[1] J. Schutz and C. Webb. A scalable X86 CPU design for 90nm process. ISSCC, 2004.

[2] A. P. Chandrakasan and R. W. Brodersen. Low Power Digital CMOS Design. Kluwer Academic Publishers, 1995.

[3] D. Liu and C. Svensson. Trading speed for low power by choice of supply and threshold voltages. IEEE Journal of Solid-State Circuits, 28(1):10-17, January 1993.

[4] C. Kim, H. Soeleman, and K. Roy. Ultra-low-power DLMS adaptive filter for hearing aid applications. IEEE Transactions on VLSI, 11(6):1058-1067, December 2003.

[5] M. Eisele, , et al. The impact of intra-die device parameter variations on path delays and on the design for yield of low voltage digital circuits. IEEE Transactions on VLSI, 5(4):360-368, December 1997.

[6] D. Boning and S. Nassif. Models of process variations in device and interconnect. In A. Chandrakasan, W. J. Bowhill, and F. Fox, editors, Design of High-Performance Microprocessor Circuits. IEEE Press, New York, NY, 2001.

[7] http://www-device.eecs.berkeley.edu/ ptm/.

[8] D. Lee, W. Kwong, D. Blaauw, and D. Sylvester. Simultaneous subthreshold and gate-oxide tunneling leakage current analysis in nanometer CMOS design. ISQED, pages 287-292, 2003.

[9] W.K. Henson et al. Analysis of leakage currents and impact on off-state power consumption for CMOS technology in the 100-nm regime. IEEE Transactions on Electron Devices, 47(2):440-447, February 2000.

[10] Y.L. Tong. The Multivariate Normal Distribution. Springer-Verlang, 1990.

[11] B.H. Calhoun, A. Wang, and A. Chandrakasan. Device sizing for minimum energy operation in subthreshold circuits. Custom Integrated Circuits Conference, 2003. 

Variations in Leakage Power and Performance

\author{
Ashish Srivastava, Saumil Shah, Kanak Agarwal, Dennis Sylvester, \\ David Blaauw, Stephen Director \\ University of Michigan, EECS Department, Ann Arbor, MI
}

\begin{abstract}
Increasing levels of process variation in current technologies have a major impact on power and performance, and result in parametric yield loss. In this work we develop an efficient gate-level approach to accurately estimate the parametric yield defined by leakage power and delay constraints, by finding the joint probability distribution function (jpdf) for delay and leakage power. We consider inter-die variations as well as intra-die variations with correlated and random components. The correlation between power and performance arise due to their dependence on common process parameters and is shown to have a significant impact on yield in high-frequency bins. We also propose a method to estimate parametric yield given the power/delay jpdf that is much faster than numerical integration with good accuracy. The proposed approach is implemented and compared with Monte Carlo simulations and shows high accuracy, with the yield estimates achieving an average error of $2 \%$.

Keywords: Yield, Variability, Leakage, Correlation
\end{abstract}

\title{
References
}

[1] A. Chandrakasan, W.J. Bowhill, and F. Fox, Design of high-performance microprocessor circuits, IEEE Press, 2001.

[2] T. Karnik, S. Borkar, and V. De, "Sub-90 nm technologies challenges and opportunities for CAD," ACM/IEEE ICCAD, pp. 203-206, 2002.

[3] X. Bai, et al., "Uncertainty aware circuit optimization," ACM/IEEE DAC, pp.58-63, 2002.

[4] S. Raj, S. Vrudhula, and J. Wang, "A methodology to improve timing yield in the presence of process variations," ACM/IEEE DAC, pp. 448-453, 2004.

[5] S. Choi, B. Paul and K. Roy, "Novel sizing algorithm for yield improvement under process variation in nanometer technology," IEEE/ACM DAC, pp. 454-459, 2004.

[6] A. Srivastava, D. Sylvester, and D. Blaauw, "Statistical optimization of leakage power considering process variations using dual-Vth and sizing," ACM/IEEE DAC, pp. 773-778, 2004.

[7] H. Chang and S. S. Sapatnekar, "Statistical timing analysis considering spatial correlations using a single PERTlike traversal," ACM/IEEE ICCAD, pp. 621-625, 2003.

[8] C. Viswesweriah et al, "First-order incremental block-based statistical timing analysis," ACM/IEEE DAC, 2004.

[9] A. Agarwal et al., "Statistical timing analysis using bounds and selective enumeration," IEEE TCAD, pp. 12431260, Sept. 2003.

[10] S. Bhardwaj, S. Vrudhula, and D. Blaauw, "TAU: Timing analysis under uncertainty," $A C M / I E E E I C C A D$, pp. 615-620, Nov. 2003.

[11] A. Devgan and C. Kashyap, "Block-Based statistical timing analysis with uncertainty," $A C M / I E E E$ ICCAD, pp. 607-614, 2003.

[12] M. Orshansky and A. Bandyopadhyay, "Fast statistical timing analysis handling arbitrary delay correlations," $A C M / I E E E D A C$, pp. 337-342, 2004.

[13] R.R. Rao, et al., "Statistical analysis of subthreshold leakage current for VLSI circuits," IEEE Trans. VLSI Systems, pp.131-139, Feb. 2004.

[14] R.R. Rao, et al., "Parametric yield estimation considering leakage variability," $A C M / I E E E D A C$, pp. 442-447, 2004.

[15] K. Bowman et al., "Impact of die-to-die and within-die Parameter Fluctuations on the Maximum Clock Frequency Distribution for Gigascale Integration”, IEEE JSSC, pp.183-190, Feb. 2002.

[16] S. Duvall, "Statistical Circuit Modeling and Optimization,” Workshop on Statistical Metrology, pp.56-63, 2000.

[17] S. D. Samaan, "The impact of device parameter variation on the frequency and performance of VLSI chips," ACM/IEEE ICCAD, pp. 343-346, 2004. 
[18] A. Papoulis, Probability, Random Variables, and Stochastic Processes, McGraw-Hill Inc, New York, 1991. [19] S. Tsukiyama, M. Tanaka, and M. Fukui, "A new statistical static timing analyzer considering correlation between delays," Proc. TAU, pp. 27-33, Dec. 2002.

[20] C. Clark, "The greatest of a finite set of random variables," Operations Research, vol. 9, pp. 85-91, 1961.

[21] A. Abu-Dayya and N. Beaulieu, "Outage probabilities in the presence of correlated lognormal interferers," IEEE Trans. Vehicular Technology, pp.164-173, Feb. 1994.

[22] S.C. Schwartz and Y.S. Yeh, "On the distribution function and moments of power sums with lognormal components," Bell Systems Technical Journal, vol.61, pp.1441-1462, Sep. 1982.

[23] J. H. Cadwell, "The bivariate normal integral," Biometrika, pp. 31-35, Dec. 1951.

[24] F. Brglez and H. Fujiwara, "A neutral netlist of 10 combinational benchmark circuits and a target translator in Fortran,” Proc. ISCAS, pp. 695-698, May 1989.

[25] http://www.cbl.ncsu.edu 


\title{
Leakage Minimization of Nano-Scale Circuits in the Presence of Systematic and Random Variations
}

\author{
Sarvesh Bhardwaj, Sarma B.K.Vrudhula \\ Computer Science and Engineering, Arizona State University
}

\begin{abstract}
This paper presents a novel gate sizing methodology to minimize the leakage power in the presence of process variations. The leakage and delay are modeled as posynomials functions to formulate a geometric programming problem. The existing statistical leakage model of [18] is extended to include the variations in gate sizes as well as systematic variations. We propose techniques to efficiently evaluate constraints on the $\alpha$-percentile of the path delays without enumerating the paths in the circuit. The complexity of evaluating the objective function is $\mathrm{O}(|\mathrm{N}| 2)$ and that of evaluating the delay constraints is $\mathrm{O}(|\mathrm{N}|+|\mathrm{E}|)$ for a circuit with $|\mathrm{N}|$ gates and $|\mathrm{E}|$ wires. The optimization problem is then solved using a convex optimization algorithm that gives an exact solution.
\end{abstract}

Keywords: Leakage, Statistical, Optimization, Geometric Programming

\section{REFERENCES}

[1] S. Borkar, T. Karnik, S. Narendra, J. Tschanz, A. Keshavarzi, and V. De. Parameteric variations and impact on circuits and microarchitecture. In Proc. DAC, 2003.

[2] S. Boyd, S. J. Kim, L. Vandenberghe, and A. Hassibi. A tutorial on geometric programming. Technical report, www.stanford.edu/ boyd/gp tutorial.html, 2004.

[3] C. Chen and M. Sarrafzadeh. Simultaneous voltage scaling and gate sizing for low-power design. Trans. on CAS-II: Analog and Digital Signal Processing, 49(6):400-408, 2002.

[4] S. W. Director et al. Optimization of parametric yield: A tutorial. In Proc. of CICC, pages 3.1.1-8, 1992.

[5] J. Fishburn and A. Dunlop. TILOS: a posynomial programming approach to transistor sizing. In Proc. ICCAD, pages 326-328, 1985.

[6] M. Ketkar and S. S. Sapatnekar. Standby power optimization via transistor sizing and dual threshold voltage assignment. In Proc. of ICCAD, pages 375 - 378, 2002.

[7] D. Lee, H. Deogun, D. Blaauw, and D. Sylvester. Simultaneous state, vt and tox assignment for total standby power minimization. In Proc. of DATE, 2004.

[8] C. Long and L. He. Distributed sleep transistors network for power reduction. In Proc of DAC, pages 181 - 186, 2003.

[9] V. Mehrotra, S. Nassif, D. Boning, and J. Chung. Modeling the effects of manufacturing variation on high-speed microprocessor interconnect performance. In International Electronic Devices Meeting, pages 767-770. IEEE, Dec 1998.

[10] V. Mehrotra et al. A methodology for modeling the effects of systematic within-die interconnect and device variation on circuit performance. In Proc. of DAC, pages 172-175, 2000.

[11] S. Mukhopadhyay, A. Raychowdhury, and K. Roy. Accurate estimation of total leakage current in scaled CMOS logic circuits based on compact current modeling. In Proc. of DAC, pages 169-174, 2003.

[12] S. Narendra et al. Full-chip subthreshold leakage power prediction and reduction techniques for sub-0.18- $\mu / \mathrm{m}$ CMOS. Journal of Solid-State Circuits, 39(2):501-510, Feb 2004.

[13] S. R. Nassif. Modeling and analysis of manufacturing variations. In IEEE Conf. on Custom Integrated Circuits, pages 223-228, 2001.

[14] C. Neau and K. Roy. Optimal body bias selection for leakage Improvement and Process Compensation over different technology generations. In ISLPED, pages 116-121, 2003.

[15] M. Orshansky, L. Milor, P. Chang, K. Keutzer, and C. Hu. Impact of spatial intrachip gate length variability on the performance of high-speed digital circuits. In IEEE Transactions on CAD, volume 21, May 2002.

[16] E. L. Peterson. Geometric programming. SIAM Review, 18(1):1-51, Jan 1976.

[17] S. Raj, S. Vrudhula, and J. M. Wang. A methodology to improve timing yield in the presence of process variations. In Proc. of DAC, pages 448-453, 2004.

[18] R. Rao, A. Devgan, D. Blaauw, and D. Sylvester. Parametric yield estimation considering leakage variability. In Proc. Of DAC, pages 442-447, 2004. 
[19] S. S. Sapatnekar, V. B. Rao, P. M. Vaidya, and S.-M. Kang. An exact solution to the transistor sizing problem for CMOS circuits using convex optimization. Trans. on CAD, 12(11):1621-1634, Nov 1993.

[20] S. Sirichotiyakul et al. Stand-by power minimization through simultaneous threshold voltage selection and circuit sizing. In Proc. of DAC, pages 436 - 441, 1999.

[21] A. Srivastava, D. Sylvester, and D. Blaauww. Power minimization using simultaneous gate sizing, dual-vdd and dual-vth assignment. In Proc. of DAC, pages 783-787, 2004.

[22] A. Srivastava et al. Statistical optimization of leakage power considering process variations using dual-vth and sizing. In Proc. of DAC, pages 773-778, 2004.

[23] B. E. Stine, D. S. Boning, and J. E. Chung. Analysis and Decomposition of Spatial Variation in IC processes and devices. IEEE Trans. on Sem. Manuf., 10(1):24-41, Feb 1997.

[24] B. E. Stine et al. Simulating the Impact of Pattern-Dependent Poly-CD variation on circuit performance. IEEE Trans. on Sem. Man., 11(4):552-556, November 1998.

[25] M. A. Styblinski. Statistical design centering approach to minimax circuit design. In Proc. of ISCAS, pages 697-700, 1989.

[26] T. Tugbawa et al. A mathematical model of pattern dependencies in Cu CMP processes. In Proc. CMP Symp. Electrochem. Soc. Meeting, pages 605-615, 1999.

[27] C. Visweswariah, K. Ravindran, K. Kalafala, S. G. Walker, and S. Narayan. First-order incremental blockbased statistical timing analysis. In Proc. of DAC, pages 331-336, 2004.

[28] R. von Mises. Mathematical Theory of Probability and Statistics. Academic Press, 1964.

[29] Q. Wang and S. Vrudhula. Algorithms for minimizing standby power in deep submicrometer, dual-vt cmos circuits. Trans. on CAD, 21(3):306-318, 2002. 


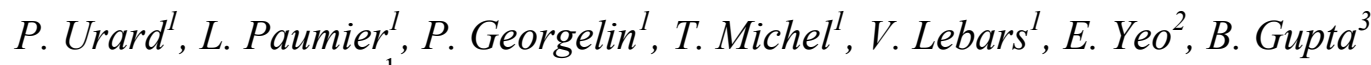 \\ ${ }^{1}$ STMicroelectronics, Crolles, France \\ ${ }^{2}$ STMicroelectronics, San Jose, CA \\ ${ }^{3}$ W5Networks, Palo Alto, CA
}

\begin{abstract}
A DVB-S2 compliant codec is implemented in both $130 \mathrm{~nm}-8 \mathrm{M}$ and $90 \mathrm{~nm}-7 \mathrm{M}$ low-leakage CMOS technologies. The system includes encoders and decoders for both Low-Density Parity Check (LDPC) codes and serially concatenated BCH codes. All requirements of the DVB-S2 standard are supported including code rates between $1 / 4$ and 9/10, block sizes of either 16,200 bits or 64,800 bits, and four digital modulation options. The $130 \mathrm{~nm}$ core design occupies $49.6 \mathrm{~mm} 2$ and operates at $200 \mathrm{MHz}$, while the $90 \mathrm{~nm}$ core design occupies $15.8 \mathrm{~mm} 2$ and operates at $300 \mathrm{MHz}$.
\end{abstract}

Keywords: DVB-S2, LDPC, FEC (forward error correction)

\title{
REFERENCES
}

[1] Draft ETSI EN 302307 V1.1.1, 2004-06

[2] R. Bose and D. Ray-Chaudhuri, On a class of error correcting binary group codes, Inform. Contr., vol. 3, pp. 68 69,1960

[3] R. Gallager, "Low density parity check codes," IRE Trans. Inform. Theory, vol. IT-8, pp. 21, Jan 28, 1962

[4] A. Blanksby and C. Howland, "A 690-mW 1-Gbit/s 1024-bit rate-1/2 low density parity check code decoder," IEEE J. Solid-State Circuits, vol. 37, no. 3, Mar 2002, pp. 404-412

[5] E. Yeo, et. al., "High throughput low-density parity-check architectures," Proc. IEEE Globecom 2001, pp. 30193024, San Antonio, TX, Nov 25-29, 2001

[6] P. Urard, et. al., "A Generic $350 \mathrm{Mb} / \mathrm{s}$ Turbo-Codec Based on a 16-states SISO Decoder, "Proc. IEEE ISSCC 2004, pp. 424-425, Feb 2004, San Francisco, CA

[7] P. Georgelin "Formal verification of synchronous digital systems, based on symbolic simulation". PhD Thesis, Université Joseph Fourier, France, 18 Oct 2001

[8] M. Kaufmann, P. Manolios, and J.S. Moore, "Computer-Aided Reasoning: An Approach", Kluwer Academic Publishers, June 2000.

[9] P. Urard, et al. "A 135Mbps DVB-S2 Compliant Codec based on 64800-bit LDPC and BCH Codes" Proc. IEEE ISSCC 2005, pp. 446-447, Feb 2005, San Francisco, CA 


\title{
A Design Platform for 90-nm Leakage Reduction Techniques
}

Philippe Royannez, Hugh Mair, Franck Dahan, Mike Wagner, Mark Streeter, Laurent Bouetel, Joel Blasquez, H. Clasen, G. Semino, Julie Dong, D. Scott, B. Pitts, Claudine Raibaut, Uming Ko

Texas Instruments, KILBY - BP 5 - 06271 Villeneuve-Loubet Cedex

\begin{abstract}
Methodology, EDA Flow, scripts, and documentation plays a tremendous role in the deployment and standardization of advanced design techniques. In this paper we focus not only on leakage reduction techniques but also on their deployment as a worldwide infrastructure as the addedvalue resides not only in the techniques themselves but also in the way they are implemented to build an efficient, re-usable, robust, low cost and portable platform. Techniques have been silicon proven on the 90-nm TI CMOS technology and is commonly used to design SoC with complexities over 100 Million transistors
\end{abstract}

Keywords: SoC Design, Leakage Power Management, Wireless Application processor

\section{REFERENCES}

[1] P. Royannez, H. Mair, F. Dahan, M. Wagner et. al.; "90nm Low Leakage SoC Design Techniques for Wireless Applications"; ISSCC'05, Feb 2005 


\title{
A 24 GHz Phased-Array Transmitter in $0.18 \mu \mathrm{m}$ CMOS
}

Arun Natarajan, Abbas Komijani, and Ali Hajimiri

California Institute of Technology (Caltech), Pasadena, CA, USA

\begin{abstract}
A fully-integrated 4-element phased array transmitter at $24 \mathrm{GHz}$ with on-chip PAs is demonstrated in $0.18 \mu \mathrm{m}$ CMOS. It has a beam-forming resolution of $10^{\circ}$, a peak-to-null ratio of $23 \mathrm{~dB}$, and $28 \mathrm{~dB}$ isolation between paths. Each PA can deliver up to $+14 \mathrm{dBm}$ into $50 \Omega$ in saturation. The die size is $6.8 \mathrm{~mm} \times 2.1 \mathrm{~mm}$. The transmitter bandwidth is more than $400 \mathrm{MHz}$ and supports up to $1 \mathrm{Gbit} / \mathrm{s}$ QPSK, facilitating a Gigabit wireless LAN solution.

Keywords: Wireless, Transmitters, Phased-Array, 24GHz, CMOS, IC
\end{abstract}


Cache Coherence Support for Non-Shared Bus Architecture on Heterogeneous MPSoCs

Taeweon Suh*, Daehyun Kim**, Hsien-Hsin S. Lee*
*School of Electrical and Computer Engineering, Georgia Institute of Technology, Atlanta, GA
**Microprocessor Technology Labs, Intel Corporation, Santa Clara, CA

\begin{abstract}
We propose two novel integration techniques -- bypass and bookkeeping -- in the memory controller to address the cache coherence compatibility issue of a non-shared bus heterogeneous MPSoC. The bypass approach is an inexpensive and efficient solution for computation-bound applications while the bookkeeping approach eliminating unnecessary forwarding traffic offers an alternative for bandwidth-limited applications. Our RTOS kernel simulations show up to $6.65 x$ speedup over the conventional software solution.

Keywords: Cache coherence, Inter-processor communication, Heterogeneous MPSoC, Realtime and embedded systems
\end{abstract}

\title{
REFERENCES
}

[1] L. Barroso, K. Gharachorloo, R. McNamara, A. Nowatzyk, S. Qadeer, B. Sano, S. Smith, R. Stets, and B. Verghese. Piranha: A Scalable Architecture Based on Single-Chip Multiprocessing. In Proc. of the Int'l Symp. on Computer Architecture, 2000.

[2] D. Chaiken, J. Kubiatowicz, and A. Agarwal. LimitLESS Directories: A Scalable Cache Coherence Scheme. In Proc. of the In'tl Conf. on Architectural Support of Programming Languages and Operating Systems, pages 224-234, Apr. 1991.

[3] D. E. Culler, J. P. Singh, and A. Gupta. Parallel Computer Architecture: A Hardware/Software Approach. Morgan Kaufmann Publishers, 1999.

[4] K. Gharachorloo, M. Sharma, S. Steely, and S. V. Doren. Architecture and Design of AlphaServer GS314. In Proc. Of the Int'l Conf. on Architecture Support for Programming Languages and Operating Systems, 2000.

[5] J. Kuskin, D. Ofelt, M. Heinrich, J. Heinlein, R. Simoni, K. Gharachorloo, J. Chapin, D. Nakahira, J. Baxter, M. Horowitz, A. Gupta, M. rosenblum, and J. Hennessy. The Stanford FLASH Multiprocessor. In Proc. of the Int'1 Symp. on Computer Architecture, 1994.

[6] J. Laudon and D. Lenoski. The SGI Origin: A ccNUMA Highly Scalable Server. In Proc. of the Int'l Symp. on Computer Architecture, 1997.

[7] D. Lenoski, J. Laudon, K. Gharachorloo, A. Gupta, and J. Hennessy. The Directory-Based Cache Coherence Protocol for the DASH Multiprocessor. In Proc. of the Int'l Symp. on Computer Architecture, 1990.

[8] Mentor Graphics. Hardware/Software Co-Verification: Seamless. http://www.mentor.com/seamless.

[9] T. Suh, D. M. Blough, and H.-H. S. Lee. Supporting Cache Coherence in Heterogeneous Multiprocessor Systems. In Proc. of the Conf. on Design, Automation and Test in Europe, 2004.

[10] T. Suh, H.-H. S. Lee, and D. M. Blough. Integrating Cache Coherence Protocols for Heterogeneous Multiprocessor Systems, Part 1. IEEE Micro, July/August 2004.

[11] T. Suh, H.-H. S. Lee, and D. M. Blough. Integrating Cache Coherence Protocols for Heterogeneous Multiprocessor Systems, Part 2. IEEE Micro, September/October 2004.

[12] D.-S. Sun, D. M. Blough, and V. Mooney. Atalanta: A New Multiprocessor RTOS Kernel for System-on-aChip Applications. Technical Report GIT-CC-02-19, CERCS, Georgia Institute of Technology, 2002.

[13] W. Wolf. The Future of Multiprocessor Systems-on-Chips. In Proc. of the 42th Design Automation Conference, 2004.

[14] S. Yoo, G. Nicolescu, D. Lyonnard, A. Baghdadi, and A. A. Jerraya. A Generic Wrapper Architecture for Multi-Processor SoC Cosimulation and Design. In Proc. Of the Int'1 Symp. on Hardware/Software Codesign, 2001. 


\title{
A Low Latency Router Supporting Adaptivity for On-Chip Interconnects
}

Jongman Kim, Dongkook Park, T. Theocharides, N. Vijaykrishnan and Chita R. Das Department of Computer Science and Engineering, The Pennsylvania State University University Park, PA

\begin{abstract}
The increased deployment of System-on-Chip designs has drawn attention to the limitations of on-chip interconnects. As a potential solution to these limitations, Networks-on-Chip (NoC) have been proposed. The NoC routing algorithm significantly influences the performance and energy consumption of the chip. We propose a router architecture which utilizes adaptive routing while maintaining low latency. The two-stage pipelined architecture uses look ahead routing, speculative allocation, and optimal output path selection concurrently. The routing algorithm benefits from congestion-aware flow control, making better routing decisions. We simulate and evaluate the proposed architecture in terms of network latency and energy consumption. Our results indicate that the architecture is effective in balancing the performance and energy of NoC designs.
\end{abstract}

Keywords: Networks-On-Chip, Adaptive Routing, Interconnection Networks

\section{REFERENCES}

[1] Berkeley predictive technology model. http://www-device.eecs.berkeley.edu/ptm/.

[2] The nostrum backbone project. http://www.imit.kth.se/info/FOFU/Nostrum/.

[3] Stanford-bologna netchip project. http://akebono.stanford.edu/users/nanni/research/net/netchip/.

[4] L. Benini and G. D. Micheli. Networks on Chips: A New SoC Paradigm. IEEE Computer, 35(1):70-78, 2002.

[5] W. J. Dally and B. Towles. Route Packets, Not Wires: On-Chip Interconnection Networks. In Proceedings of the 38th Design Automation Conference, June 2001.

[6] W. J. Dally and B. Towles. Principles and Practices of Interconnection Networks. Morgan Kaufmann, 2003.

[7] J. Dielissen and et. al. Concepts and implementation of the Philips network-on-chip. In IP-Based SOC Design, Nov. 2003.

[8] N. Eisley and L.-S. Peh. High-level power analysis for on-chip networks. In Proceedings of CASES, pages 104115. ACM Press, 2004.

[9] P. Guerrier and A. Greiner. A generic architecture for on-chip packet-switched interconnections. In Proc. of DATE, pages 250-256. ACM Press, 2000.

[10] M. Horowitz, R. Ho, and K. Mai. The future of wires. In Proc. of SRC Conference, 1999.

[11] J. Hu and R. Marculescu. DyAD - Smart Routing for Networks-on-Chip. In In Proceedings of the 41 'st ACM/IEEE DAC, 2004.

[12] A. Jalabert, S. Murali, L. Benini, and G. D. Micheli. xpipes compiler: a tool for instantiating application specific networks on chip. In Proc. of DATE, pages 884-889, 2004.

[13] R. M. Jingcao Hu. Energy-aware mapping for tile-based noc architectures under performance constraints. In Proc. of ASPDAC, January 2003.

[14] R. Mullins and et. al. Low-latency virtual-channel routers for on-chip networks. In Proc. of the 31st ISCA, page 188. IEEE Computer Society, 2004.

[15] S. Y. Nam and D. K. Sung. Decomposed crossbar switches with multiple input and output buffers. In Proc. of IEEE GLOBECOM, 2001.

[16] L.-S. Peh and W. J. Dally. A Delay Model and Speculative Architecture for Pipelined Routers. In Proceedings of the 7th International Symposium on High-Performance Computer Architecture, 2001.

[17] P. Rickert. Problems or opportunities? beyond the 90nm frontier, 2004. ICCAD - Keynote Address.

[18] L. Shang, L.-S. Peh, A. Kumar, and N. K. Jha. Thermal Modeling, Characterization and Management of OnChip Networks. In Proc. of the $37^{\text {th }}$ MICRO, 2004.

[19] H.-S. Wang, L.-S. Peh, and S. Malik. Power-Driven Design of Router Microarchitectures in On-Chip Networks. In Proceedings of the 36th MICRO, November 2003. 


\title{
Floorplan-Aware Automated Synthesis of Bus-based Communication Architectures
}

\author{
Sudeep Pasricha†, Nikil Dutt†, Elaheh Bozorgzadeh $†$, Mohamed Ben-Romdhane $\neq$ \\ $\dagger$ Center for Embedded Computer Systems, University of California, Irvine, CA \\ †Conexant Systems Inc., Newport Beach, CA
}

\begin{abstract}
As System-on-Chip (SoC) designs become more complex, it is becoming harder to design communication architectures to handle the ever increasing volumes of inter-component communication. Manual traversal of the vast communication design space to synthesize a communication architecture that meets performance requirements becomes infeasible. In this paper, we address this problem by proposing an automated approach for synthesizing costeffective, bus-based communication architectures that satisfy the performance constraints in a design. Our synthesis flow also incorporates a high-level floorplanning and wire delay estimation engine to evaluate the feasibility of the synthesized bus architecture and detect timing violations early in the design flow. We present case studies of network communication SoC subsystems for which we synthesized bus architectures, detected timing violations and generated core placements in a matter of hours instead of several days it took for a manual effort.
\end{abstract}

Keywords: Communication Synthesis, Systems-on-Chip

\section{REFERENCES}

[1] D. Sylvester and K. Keutzer, "Getting to the bottom of deep submicron", In Proc. of ICCAD 1998

[2] Flynn, "AMBA: enabling reusable on-chip designs" In IEEE Micro, 1997

[3] IBM CoreConnect http://www.chips.ibm.com/products/powerpc/cores

[4] Open Core Protocol International Partnership (OCP-IP). OCP datasheet, http://www.ocpip.org

[5] S. Pasricha, N. Dutt, M. Ben-Romdhane, "Fast Exploration of Busbased On-chip Communication Architectures", In Proc. of CODESISSS 2004

[6] S. Narayan, D. Gajski, "Synthesis of system level bus interfaces", In Proc. of DATE 1994

[7] J. Daveau, et al "Protocol selection and interface generation for HWSW codesign", In IEEE Trans. on VLSI System, Vol. 5, No. 1, March 1997

[8] M. Gasteier, M. Glesner "Bus-based communication synthesis on system level", In ACM TODAES, January 1999

[9] K. K. Ryu, V. J. Mooney III “Automated Bus Generation for Multiprocessor SoC Design”, In Proc. of DATE 2003

[10] A. Pinto et al "Constraint-driven communication synthesis", DAC 2002

[11] D. Lyonnard et al "Automatic generation of application-specific architectures for heterogeneous multiprocessor system-on-chip", DAC 2001

[12] K. Lahiri et al, "Efficient exploration of the SoC communication architecture design space", In Proc. of ICCAD 2000

[13] C. Shin, et al "Fast Exploration of Parameterized Bus Architecture for Communication-Centric SoC Design", In Proc. of DATE 2004

[14] N. Thepayasuwan, A. Doboli "Layout Conscious Bus Architecture Synthesis for Deep Submicron Systems on Chip", In Proc. of DATE 2004

[15] M. Drinic et al. "Latency-guided on-chip bus network design", In Proc. of ICCAD 2000

[16] J. Hu et al, "System-Level Point-to-Point Communication Synthesis Using Floorplanning Information", In Proc. of ASP-DAC 2002

[17] R. P. Dick, N. K. Jha "MOCSYN: multiobjective core-based singlechip system synthesis", In Proc. of DATE 1999

[18] R. A. Bergamaschi et al "SEAS: a system for early analysis of SoCs", In Proc. of CODES-ISSS 2003

[19] S. N. Adya, I. L. Markov, "Fixed-outline Floorplanning: Enabling Hierarchical Design", In IEEE Trans TVLSI, Dec. 2003

[20] A. E. Caldwell, et al, "On Wirelength Estimations for Row-based Placement", In IEEE Trans. on ICCAD, vol.18, (no.9), IEEE, Sept 1999 
[21] J. Cong, D. Z. Pan, "Interconnect Performance Estimation Models for Design Planning”, In IEEE Trans. on ICCAD, Vol 20, No. 6, June 2001

[22] Semiconductor Industry Association, "National Technology Roadmap for Semiconductors", SIA 1997

[23] SystemC initiative. www.systemc.org

[24] S. Pasricha, "Transaction Level Modeling of SoC with SystemC 2.0", In Proc. of Synopsys User Group Conference (SNUG), 2002

[25] AMBA AXI Specification www.arm.com/armtech/AXI

[26] S. Pasricha, N. Dutt, M. Ben-Romdhane, "Extending the Transaction Level Modeling Approach for Fast Communication Architecture Exploration", In Proc. of DAC 2004 


\title{
FLEXBUS: A High-Performance System-on-Chip Communication Architecture with a
} Dynamically Configurable Topology

\author{
Krishna Sekar ${ }^{1}$, Kanishka Lahiri ${ }^{2}$, Anand Raghunathan ${ }^{2}$, Sujit Dey ${ }^{1}$ \\ ${ }^{1}$ Dept. of ECE, UC San Diego, CA \\ ${ }^{2}$ NEC Laboratories America, Princeton, NJ
}

\begin{abstract}
In this paper, we describe FLEXBUS, a flexible, high-performance on-chip communication architecture featuring a dynamically configurable topology. FLEXBUS is designed to detect runtime variations in communication traffic characteristics, and efficiently adapt the topology of the communication architecture, both at the system-level, through dynamic bridge by-pass, as well as at the component-level, using component re-mapping. We describe the FLEXBUS architecture in detail and present techniques for its run-time configuration based on the characteristics of the on-chip communication traffic. The techniques underlying FLEXBUS can be used in the context of a variety of on-chip communication architectures. In particular, we demonstrate its application to AMBA AHB, a popular commercial on-chip bus. Detailed experiments conducted on the FLEXBUS architecture using a commercial design flow, and its application to an IEEE 802.11 MAC processor design, demonstrate that it can provide significant performance gains as compared to conventional architectures (up to $31.5 \%$ in our experiments), with negligible hardware overhead.
\end{abstract}

Keywords: Communication architectures, On-chip bus

\section{REFERENCES}

[1] "CoreConnect BusArchitecture." http://www.chips.ibm.com/products/coreconnect/.

[2] "AMBA 2.0 Specification." http://www.arm.com/armtech/AMBA.

[3] D. Wingard and A. Kurosawa, "Integration Architecture for System-on-a-Chip Design," in Proc. Custom Integrated Circuits Conf., pp. 85-88, 1998.

[4] A. Adriahantenaina, H. Charlery, A. Greiner, L. Mortiez, and C. A. Zeferino, "SPIN: A Scalable, Packet Switched, On-ChipMicro-Network," in Proc. Design Automation \& Test Europe (DATE) Conf., pp. 70-73, 2003.

[5] S. Han, A. Baghdadi, M. Bonaciu, S. Chae, and A. A. Jerraya, "An Efficient Scalable and Flexible Data Transfer Architecture for Multiprocessor SoC with Massive Distributed Memory," in Proc. Design Automation Conf., pp. 250-255, June 2004.

[6] R. Yoshimura, K. T. Boon, T. Ogawa, S. Hatanaka, T. Matsuoka, and K. Taniguchi, "DS-CDMAWired BusWith Simple Interconnection Topology for Parallel Processing System LSIs," in Proc. Int. Solid-State Circuits Conf., pp. 370-371, 2000.

[7] K.Lahiri, A.Raghunathan, and S.Dey, "Design of High-Performance System-on-Chips Using Communication Architecture Tuners," IEEE Trans. on CAD, vol. 23, no. 6, pp. 919-932, 2004.

[8] T. Meyerowitz, C. Pinello, and A. Sangiovanni-Vincentelli, "A Tool for Describing and Evaluating Hierarchical Real-Time Bus Scheduling Policies," in Proc. Design Automation Conf., pp. 312-317, June 2003.

[9] J. Hu and R. Marculescu, "DyAD - Smart Routing For Networks-on-Chip," in Proc. Design Automation Conf., pp. 260-263, June 2004.

[10] A. Pinto, L. P. Carloni, and A. Sangiovanni-Vincentelli, "Constraint-Driven Communication Synthesis," in Proc. Design Automation Conf., pp. 783-788, June 2002.

[11] S.Pasricha, N.Dutt, and M.B.Romdhane, "Fast Exploration of Bus-based On-chip Communication Architectures," in Proc. Int. Symp. HW/SW Codesign, Sept. 2004.

[12] "Wireless LAN Medium Access Control (MAC) and Physical Layer (PHY) Specifications." IEEE Computer Society LAN/MAN Standards Committee, IEEE Std 802.11-1999 Edition.

[13] L.Benini, A.Bogliolo, G.Paleologo, and G.D.Micheli, "Policy Optimization for Dynamic Power Management," IEEE Trans. on CAD, vol. 18, pp. 813-833, June 1999.

[14] F. Douglis, P.Krishnan, and B. Bershad, "Adaptive Disk Spin-Down Policies for Mobile Computers," in USENIX Symp. Mobile and Location Independent Computing, pp. 121-137, Apr. 1995.

[15] "Synopsys DesignWare Intellectual Property." http://www.synopsys.com/products/designware/designware.html. 
[16] "Modelsim 5.7e." http://www.model.com.

[17] W. Dai, L. Wu, and S. Zhang, "UCSC Floorplanning Tool." http://www.soe.ucsc.edu/research/surf/GSRC/ progress.html.

[18] "CB-12." http://www.necel.com/cbic/en/cb12/cb12.html.

[19] H. B. Bakoglu, Circuits, Interconnections, and Packaging for VLSI. Addison-Wesley, Menlo Park, CA, 1990.

[20] “Design Compiler 2003.12, Synopsys Inc..” http://www.synopsys.com/products/logic/design_compiler.html. 


\title{
Trafffic Shaping for an FPGA based SDRAM Controller with Complex QoS Requirements
}

Sven Heithecker, Rolf Ernst

Institute of Computer and Communication Network Engineering, Technical University of Braunschweig

\begin{abstract}
Today high-end video and multimedia processing applications require huge amounts of memory. For cost reasons, the usage of conventional dynamic RAM (SDRAM) is preferred. However, SDRAM access optimization is a complex task, especially if multi-stream access with different QoS requirements is involved. In [8], a multi-stream DDR-SDRAM controller IP covering combinations of low latency requirements for processor cache access, hard realtime constraints for periodic video signals and hard real-time bursty accesses for video coprocessors was described. To handle these contradictory QoS requirements at high system performance, a combination of a 2-stage scheduling algorithm and static priorities were used. This paper describes an additional flow control which enhances the overall performance. Experiments with an FPGA based high-end video platform demonstrate the superiority of this architecture.
\end{abstract}

Keywords: SDRAM, memory access, QoS, trafffic shaping, priorities, flow control, FPGA

\section{REFERENCES}

[1] http://microlib.org.

[2] http://www.discreet.com.

[3] http://www.flexfilm.org.

[4] http://www.ida.ing.tu-bs.de: svenh/.

[5] http://www.quantel.com.

[6] http://www.thomsonbroadcast.com.

[7] AHN, J. H., DALLY, W. J., KHAILANY, B., KAPASI, U. J., AND DAS, A. Evaluating the Imagine Stream Architecture. SIGARCH Comput. Archit. News 32, 2 (2004), 14.

[8] HEITHECKER, S., DO CARMO LUCAS, A., AND ERNST, R. A Mixed QoS SDRAM Controller for FPGABased High-End Image Processing. In Workshop on Signal Processing Systems Design and Implementation (2003), IEEE, p. TP.11.

[9] KHAILANY, B., DALLY, W. J., AND RIXNER, S. Imagine: Media Processing with Streams. IEEE Micro (March/April 2001), 35.46.

[10] LEE, C., POTKONJAK, M., AND MANGIONE-SMITH, W. H. Mediabench: a Tool for Evaluating and Synthesizing Multimedia and Communicatons Systems. In International Symposium on Microarchitecture (1997), pp. 330.335.

[11] LEITJEN, J. A. J., VAN MEERBERGEN, J. L., AND TIMMER, A. H. PROPHID: a Heterogeneous MultiProcessor Architecture for Multimedia. In International Conference on Computer Design (October 1997), pp. 164.169.

[12] MISHRA, P., GRUN, P., AND DUTT, N. Processor-Memory Co-Exploration driven by a Memory-Aware Architecture Description Language. In $14^{\text {th }}$ International Conference on VLSI Design (Jan 2001).

[13] PANDA, P., CATTHOOR, F., AND DUTT, N. Data and Memory Optimization Techniques for Embedded Systems. 140.206.

[14] RIXNER, S., DALLY, W. J., AND KAPASI, U. J. Memory Access Scheduling. In International Symposium on Computer Architecture (2000), pp. 128.138.

[15] SONICS, INC. Sonics SiliconBackplane MicroNetwork Overview.

[16] TINDELL, K. W. An Extendible Approach for Analysing Fixed Priority Hard Real-Time Systems. 133.152.

[17] VERHAEGH, W., LIPPENS, P., AND AARTS, E. Multi-dimensional periodic scheduling: model and complexity. Springer Verlag, pp. 226.235.

[18] WEBER, W.-D. Sonics MemMax Memory Scheduler.

[19] WU, P.-C., AND CHEN, L.-G. An Efficient Architecture for Two-Dimensional Discrete Wavelet Transform. IEEE Transactions on circuits and systems for video technology 11, 4 (April 2001). 


\title{
Microarchitecture-Aware Floorplanning Using a Statistical Design of Experiments Approach
}

Vidyasagar Nookala, Ying Chen, David J. Lilja, Sachin S. Sapatnekar

Department of Electrical and Computer Engineering, University of Minnesota, Minneapolis, MN

\begin{abstract}
Since across-chip interconnect delays can exceed a clock cycle in nanometer technologies, it has become essential in high performance designs to add flip-flops on wires with multi-cycle delays. Although such a wire pipelining strategy allows higher operating frequencies, it can reduce the delivered performance of a microarchitecture, since the extra flip-flops inserted may increase the operation latencies and stall cycles. Moreover, the addition of latencies on some wires can have a large impact on the overall performance while other wires are relatively insensitive to additional latencies. This varying sensitivity suggests the need for a throughput-aware strategy for pipelining the interconnects that interacts closely with the physical design step, which determines the lengths of these multicycle wires. We use a statistical design of experiments strategy based on a multifactorial design, which intelligently uses a limited number of simulations to rank the importance of the wires. When applied at the floorplanning level, our results show improvements both in the overall system performance and in the total wire length when compared with an existing technique.
\end{abstract}

Keywords: Wire pipelining, Microarchitecture, Floorplanning

\section{REFERENCES}

[1] S. Borkar, "Obeying Moore's law beyond 0.18 micron," in Proc. IEEE ASIC/SOC, pp. 26-31, Sep. 2000.

[2] P. Cocchini, "Concurrent flip-flop and repeater insertion for high performance integrated circuits," in Proc. IEEE/ACM ICCAD, pp. 268-273, Nov. 2002.

[3] S. Hassoun et al., "Optimal buffered routing path constructions for single and multiple clock domain systems," in Proc. IEEE/ACMICCAD, pp. 247-253, Nov. 2002.

[4] V. Nookala and S. S. Sapatnekar, "Correcting the functionality of a wirepipelined circuit," in Proc. ACM/IEEE $D A C$, pp. 570-575, Jun. 2004.

[5] L. Scheffer, "Methodologies and tools for pipelined on-chip interconnect," in Proc. IEEE ICCD, pp. 152-157, Oct. 2002.

[6] D. C. Burger and T.M. Austin, "The SimpleScalar tool set, version 2.0," Technical Report CS-TR-97-1342, The University ofWisconsin, Madison, Jun. 1997.

[7] J. L. Henning, "SPEC CPU 2000: Measuring CPU performance in the new millennium," IEEE Computers, vol. 33, pp. 28-55, Jul. 2000.

[8] C. Long et al., "Floorplanning optimization with trajectory piecewise-linear model for pipelined interconnects," in Proc. ACM/IEEE DAC, pp. 640-645, Jun. 2004.

[9] M. Ekpanyapong et al., "Profile-guided microarchitectural floorplanning for deep submicron processor design," in Proc. ACM/IEEE DAC, pp. 634-639, Jun. 2004.

[10] A. Jagannathan et al., "Microarchitecture evaluation with floorplanning and interconnect pipelining," in Proc. ACM/IEEE ASPDAC, pp. 32-35, Jan. 2005.

[11] D. C. Montgomery, Design and analysis of experiments. New York, NY: John Wiley, 1991.

[12] A. J. KleinOsowski and D. J. Lilja, "MinneSPEC: A new SPEC benchmark workload for simulation-based computer architecture research," IEEE Computer Architecture Letters, vol. 1, Jun. 2002.

[13] J. Cong et al., "Microarchitecture evaluation with physical planning," in Proc. ACM/IEEE DAC, pp. 32-35, Jun. 2003.

[14] J. Yi et al., "A statistically rigorous approach for improving simulation methodology," in Proc. ACM HPCA, pp. 281-291, Feb. 2003.

[15] R. Plackett and J. Burman, "The design of optimum multifactorial experiments," Biometrika, vol. 33, pp. 305325, Jun. 1956.

[16] C. F. J. Wu and M. Hamada, Experiments: Planning, analysis, and parameter design optimization. New York, NY: John Wiley, 2000. 
[17] S. N. Adya and I. L. Markov, "Fixed-outline floorplanning through better local search," in Proc. IEEE ICCD, pp. 228-334, Oct. 2001.

[18] J. Cong, "An interconnect-centric design flow for nanometer technologies," Proc. IEEE, vol. 89, pp. 505-528, Apr. 2001.

[19] M. Ekpanyapong. Private communication, 2004. 


\title{
Timing-Driven Placement by Grid-Warping
}

Zhong Xiu, Rob A. Rutenbar

Dept. of ECE, Carnegie Mellon University, Pittsburgh, Pennsylvania, 15213 USA

\begin{abstract}
Grid-warping is a recent placement strategy based on a novel physical analogy: rather than move the gates to optimize their location, it elastically deforms a model of the 2-D chip surface on which the gates have been coarsely placed via a standard quadratic solve. In this paper, we introduce a timing-driven grid-warping formulation that incorporates slack-sensitivity-based net weighting. Given inevitable concerns about wirelength and runtime degradation in any timingdriven scheme, we also incorporate a more efficient net model and an integrated local improvement ("rewarping") step. An implementation of these ideas, WARP2, can improve worst-case negative slack by $37 \%$ on average, with very modest increases in wirelength and runtime.
\end{abstract}

Keywords: Algorithms, Placement

\section{References}

[1] S. Kirkpatrick, C. D. Gelatt Jr., and M. P. Vecchi, “Optimization by simulated annealing," Science, vol. 220, no. 4598, 13 May 1983.

[2] R. S. Tsay, E. Kuh, C. P Hsu, "PROUD: A sea-of-gates placement algorithm," IEEE Design \& Test of Computers, vol.5, Dec. 1988.

[3] Kleinhans, G. Sigl, F. Johannes, and K. Antreich, "Gordian: VLSI placement by quadratic programming and slicing optimization," IEEE Trans. CAD, vol. 10, no.3, March 1991.

[4] K. Doll, F. M. Johannes, K. J. Antreich, "Iterative placement improvement by network flow methods," Proc. IEEE Trans. CAD, vol. 13, no. 10, Oct 1994.

[5] H. Eisenmann, F. M. Johannes, "Generic global placement and floorplanning," Proc ACM/IEEE DAC, June 1998.

[6] J. Vygen, “Algorithms for large-scale flat placement," Proc ACM/IEEE DAC, June 1997.

[7] G. Karypis, R. Agarwal, V. Kumar, S. Shekhar, "Multilevel hypergraph partitioning: Applications in VLSI design," Proc ACM/IEEE DAC, June 1997.

[8] T. F. Chan, J. Cong, T. Kong, J. R. Shinner, "Multilevel optimization for large-scale circuit placement.," Proc. ACM/IEEE ICCAD, Nov. 2000.

[9] T. F. Chan, J. Cong, T. Kong, J. R. Shinner, K. Sze, “An enhanced multi-level algorithm for circuit placement,” Proc. ACM/IEEE ICCAD, Nov. 2003

[10] G. Sigl, K. Doll, F. M. Johannes, “Analytical placement: A linear or a quadratic objective function?" Proc. ACM/IEEE DAC, June 1991.

[11] A. Caldwell, A. Kahng, I. Markov, "Can recursive bisection alone produce routable placements?" Proc. ACM/IEEE DAC, June 2000.

[12] C. Chang, J. Cong, M. Xie, "Optimality and scalability study of existing placement algorithms," Proc. ASP$D A C, 2003$.

[13] N. Viswanathan, C. C.-N. Chu, "FastPlace: Efficient analytical placement using cell shifting, iterative local refinement and a hybrid net model," Proc. ACM ISPD, April 2004.

[14] Z. Xiu, J. D. Ma, S. M. Fowler, R. A. Rutenbar, "Large-scale placement by grid-warping," Proc. ACM/IEEE $D A C$, June 2004.

[15] P. Villarrubia, "Important considerations for modern VLSI chips," Proc. ACM ISPD, April 2003.

[16] C. J. Alpert, "The ISPD98 circuit benchmark suite," Proc. ACM ISPD, April 1998.

[17] M. Wang, X. Yang, M. Sarrafzadeh, "Dragon 2000: Fast standard-cell placement for large circuits," Proc. ACM/IEEE ICCAD, Nov. 2000.

[18] S. Ou, M. Pedram, "Timing-driven placement based on partitioning with dynamic cut-net control," Proc. ACM/IEEE DAC, June 2000.

[19] K. Rajagopal, T. Shaked, Y. Parasuram, T. Cao, A. Chowdhary, B. Halpin, "Timing driven force directed placement with physical net constraints," Proc. ACM ISPD, April 2003. 
[20] H. Ren, D. Z. Pan, D. S. Kung, "Sensitivity guided net weighting for placement driven synthesis," Proc. ACM ISPD, April 2004.

[21] http://openeda.si2.org/.

[22] Z. Xiu, D. A. Papa, P. Chong, C. Albrecht, A. Kuehlmann, R. A. Rutenbar, I. L. Markov, "Early research experience with OpenAcess Gear: an open source development environment for physical design" Proc. ACM ISPD, April 2005.

[23] OA Gear homepage: http://openedatools.si2.org/oagear/. 


\title{
Faster and Better Global Placement by a New Transportation Algorithm
}

\author{
Ulrich Brenner, Markus Struzyna \\ Research Institute for Discrete Mathematics, University of Bonn, Bonn, Germany
}

\begin{abstract}
We present BonnPlace, a new VLSI placement algorithm that combines the advantages of analytical and partitioning-based placers. Based on (non-disjoint) placements minimizing the total quadratic netlength, we partition the chip area into regions and assign the circuits to them (meeting capacity constraints) such that the placement is changed as little as possible. The core routine of our placer is a new algorithm for the Transportation Problem that allows to compute efficiently the circuit assignments to the regions. We test our algorithm on a set of industrial designs with up to 3.6 millions of movable objects and two sets of artificial benchmarks showing that it produces excellent results. In terms of wirelength, we can improve the results of leadingedge placement tools by about $5 \%$.
\end{abstract}

Keywords: VLSI-Placement, Global Placement, Transportation Problem

\section{REFERENCES}

[1] S.N. Adya, I.L. Markov: Consistent Placement of Macro-Blocks using oorplanning and standard-cell placement. ISPD (2002) 12-17.

[2] S.N. Adya, I.L. Markov, P.G. Villarubia: On whitespace in mixed-size placement and physical synthesis. ICCAD (2003), 311-318.

[3] S.N. Adya, I.L. Markov: Combinatorial techniques for mixed-size placement. to appear in: ACM Transactions on Design Automation of Electronic Systems (2004).

[4] C.J. Alpert: The ISPD98 circuit benchmark suite. ISPD (1998) 85-90.

[5] C.J. Alpert, T. Chan., D. J.-H. Huang, I.L. Markov, K. Yan: Quadratic placement Revisited, Design Automation Conference (1997) 752-757.

[6] C.J. Alpert, A.B. Kahng: Recent directions in netlist partitioning: a survey. Integration, the VLSI Journal 19 (1995), 1-81.

[7] U. Brenner, A. Pauli, J. Vygen: Almost optimum placement legalization by minimum cost ow and dynamic programming. ISPD (2004) 2-9.

[8] M.A. Breuer: Min-cut placement. Journal of Design, Automation and Fault-Tolerant Computing 1, 4 (1977) 343382.

[9] A.E. Caldwell, A.B. Kahng, I.L. Markov: Can recursive bisection alone produce routable placements? DAC (2000) 477-482.

[10] T. Chan, J. Cong, K. Sze, K: Multilevel generalized force-directed method for circuit placement. ISPD (2005).

[11] C.C. Chang, J. Cong, M. Xie: Optimality and scalability of existing placement algorithms. ASP-DAC (2003), 621-627.

[12] C.C. Chang, J. Cong, X. Yuan: Multi-level placement for large-scale mixed-size ic designs. ASP-DAC (2003), 325-330.

[13] H. Eisenmann, F.M. Johannes: Generic global placement and oorplanning. DAC (1998), 269-274.

[14] D.J. Huang, A.B. Kahng: Partitioning-based standard cell global placement with an exact objectice. ISPD (1997), 18-25.

[15] ISPD02 benchmarks: http://vlsicad.eecs.umich.edu/BK/ISPD02bench/

[16] A. Khatkate, C. Li, A.R. Angihotri, M.C. Yildiz, S. Ono, C.-K. Koh, P. Madden.: Recursive bisection based mixed block placement, ISPD (2004), 84-89.

[17] J. Kleinhans, G. Sigl, F. Johannes, K. Antreich: GORDIAN: VLSI Placement by Quadratic Programming and Slicing Optimization, IEEE Trans. on Computer-Aided Design 10 (3), 356-365 (1991).

[18] B. Korte, J. Vygen: Combinatorial Optimization: Theory and Algorithms. Springer, Berlin 2002, second edition 2002.

[19] J.B. Orlin: A faster strongly polynomial minimum cost flow algorithm. Operations Research 41 (1993), 338350.

[20] PEKO benchmarks: http://ballade.cs.ucla.edu/pubbench/placement

[21] M. Struzyna: Analytisches Placement im VLSI-Design, Diploma thesis, University of Bonn (2004) (in German) 
[22] T. Tokuyama, J. Nakano: Efficient algorithms for the Hitchcock transportation problem. SIAM Journal on Computing 24 (1995), 563-578.

[23] N. Viswanathan, C. Chu: FastPlace: efficient analytical placement using cell shifting, iterative local refinement and a hybrid net model, ISPD(2004), $26-33$.

[24] K. Vorwerk, A. Kennings, A. Vannelli: Engineering Details of a Stable Force-Directed Placer, ICCAD (2004), $7-11$.

[25] J. Vygen: Plazierung im VLSI-Design und ein zweidimensionales Zerlegungsproblem. Ph.D thesis. University of Bonn (1997) (in German).

[26] J. Vygen: Algorithms for large-scale at placement. Design Automation Conference (1997) 746-751. 


\title{
Multilevel Full-Chip Routing for the X-Based Architecture
}

Tsung-Yi Ho*, Chen-Fong Chang*, Yao-Wen Chang**, Sao-Jie Chen**

*Department of Electrical Engineering, National Taiwan University, Taipei, Taiwan

**Graduate Institute of Electronics Engineering and Department of Electrical Engineering,

National Taiwan University, Taipei, Taiwan

\begin{abstract}
As technology advances into the nanometer territory, the interconnect delay has become a firstorder effect on chip performance. To handle this effect, the X-architecture has been proposed for high-performance integrated circuits. The X-architecture presents a new way of orienting a chip's microscopic interconnect wires with the pervasive use of diagonal routes. It can reduce the wirelength and via count, and thus improve performance and routability. Furthermore, the continuous increase of the problem size of IC routing is also a great challenge to existing routing algorithms. In this paper, we present the first multilevel framework for full-chip routing using the $\mathrm{X}$-architecture. To take full advantage of the $\mathrm{X}$-architecture, we explore the optimal routing for three-terminal nets on the X-architecture and develop a general X-Steiner tree algorithm based on the delaunay triangulation approach for the $\mathrm{X}$-architecture. The multilevel routing framework adopts a two-stage technique of coarsening followed by uncoarsening, with a trapezoid-shaped track assignment embedded between the two stages to assign long, straight diagonal segments for wirelength reduction. Compared with the state-of-the-art multilevel routing for the Manhattan architecture, experimental results show that our approach reduced wirelength by $18.7 \%$ and average delay by $8.8 \%$ with similar routing completion rates and via counts.
\end{abstract}

Keywords: Physical design, routing, multilevel optimization, Xarchitecture

\section{REFERENCES}

[1] http://www.xinitiative.org/

[2] S. H. Batterywala, N. Shenoy, W. Nicholls, and H. Zhou, "Track assignment: A desirable intermediate step between global routing and detailed routing," Proc. of Int. Conf. Computer-Aided Design, pp. 59-66, 2002.

[3] M. Berg, M. Kreveld, M. Overmars, and O. Schwarzkopf, Computational Geometry: Algorithms and Applications, $2^{\text {nd }}$ Edition, Springer-Verlag 2000.

[4] Y.-W. Chang, K. Zhu, and D. F. Wong, "Timing-driven routing for symmetrical-array-based FPGAs," Trans. on Design Automation of Electronic Systems, vol. 5, no. 3, pp. 433-450, 2000.

[5] H. Chen, C. K. Cheng, A. B. Khang, I. I. Mandoiu, Q. Wang, and B. Yao, "The Y-Architecture for on-chip interconnect: Analysis and methodology," Proc. of Int. Conf. Computer-Aided Design, pp. 13-19, 2003.

[6] H. Chen, B. Yao, F. Zhou, and C. K. Cheng, "The Y-Architecture: Yet another on-chip interconnect solution," Proc. of Asia and South Pacific Design Automation Conf., pp. 840-846, 2003.

[7] B. Choi, C. Chiang, J. Kawa, and M. Sarrafzadeh, "Routing resources consumption on M-arch and X-arch," Proc. of Int. Symp. on Circuits and Systems, 2004.

[8] J. Cong, J. Fang, and Y. Zhang, "Multilevel approach to full-chip gridless routing," Proc. of Int. Conf. Computer-Aided Design, pp. 396-403, 2001.

[9] J. Cong, M. Xie, and Y. Zhang, "An enhanced multilevel routing system," Proc. of Int. Conf. Computer-Aided Design, pp. 51-58, 2002.

[10] J. Cong and J. Shinnerl, Multilevel optimization in VLSICAD, Kluwer Academic Publishers, 2003.

[11] C. S. Coulston, "Constructing exact octagonal steiner minimal trees," Proc. of Great Lake Symp. on VLSI, pp. $1-6,2003$.

[12] M. R. Garey, R. L. Graham, and D. S. Johnson, "The complexity of computing steiner minimal trees," SIAM Journal on Applied Mathematics, pp. 835-859, 1977.

[13] A. Hashimoto and J. Stevens , "Wire routing by optimizing channel assignment within large apertures," Proc. of Design Automation Conf., pp. 155-169, 1971.

[14] T.-Y. Ho, Y.-W. Chang, S.-J. Chen, and D. T. Lee, "A fast crosstalk- and performance-driven multilevel routing system," Proc. of Int. Conf. Computer-Aided Design, pp. 382-387, 2003. 
[15] T.-Y. Ho, Y.-W. Chang, and S.-J. Chen, "Multilevel routing with antenna avoidance," Proc. of Int. Symp. on Physical Design, pp. 34-40, 2004.

[16] A. B. Kahng, I. Mandoiu, and A. Zelikovsky, "High scalable algorithms for rectilinear and octilinear steiner trees," Proc. of Asia and South Pacific Design Automation Conf., pp. 827-833, 2003.

[17] R. Kastner, E. Bozorgzadeh, and M. Sarrafzadeh, "Pattern routing: use and theory for increasing predictability and avoiding coupling," IEEE Trans. on Computer-Aided Design, pp. 777-790, 2002.

[18] C. K. Koh and P. H. Madden, "Manhattan or Non-Manhattan? A study of alternative VLSI routing architectures," Proc. of Great Lake Symp. on VLSI, pp. 47-52, 2000.

[19] C. Y. Lee, "An algorithm for path connection and its application," IRE Trans. Electronic Computer, EC-10, 1961.

[20] S.-P. Lin and Y.-W. Chang, "A novel framework for multilevel routing considering routability and performance," Proc. of Int. Conf. Computer-Aided Design, pp. 44-50, 2002.

[21] M.Paluszewski, P. Winter, and M. Zachariasen, "A new paradigm for general architecture routing," Proc. of Great Lake Symp. on VLSI, pp. 202-207, 2004.

[22] M. R. Stan, F. Hamzaoglu, and D. Garrett, "Non-manhattan maze routing," Proc. of Brazilian Symp. on Integrated Circuit Design, pp. 260-265, 2004.

[23] S. Teig, "The X Architecture: not your father's diagonal wiring," Proc. of System Level Interconnect Predicition, pp. 33-37, 2002.

[24] Q. Zhu, H. Zhou, T. Jing, X. Hong, and Y. Yang, "Efficient octilinear steiner tree construction based on spanning graphs," Proc. of Asia and South Pacific Design Automation Conf., pp. 687-690, 2004. 


\title{
Matlab Extensions for the Development, Testing and Verification of Real-Time DSP Software
}

David P. Magee

Texas Instruments, Dallas, TX

\begin{abstract}
The purpose of this paper is to present the required tools for the development, testing and verification of DSP software in Matlab. The paper motivates a DSP Simulator concept that can be combined with the MATLAB executable interface to develop, evaluate and test DSP software within a single environment. Programming guidelines and optimization results are also provided to demonstrate the effectiveness of the intrinsics software development approach.

Keywords: C Intrinsics, Matlab, DSP Software, Optimization, Verification

\section{REFERENCES}

[1] Magee, David P., "Intrinsic Modeling within Matlab to Accelerate DSP Software Development", Texas Instruments Developer's Conference, Houston, TX, August 2002.

[2] Magee, David P., "Programming the C6x Family of DSPs: A C Intrinsics Tutorial", Texas Instruments Developer's Conference, Houston, TX, February 2005.

[3] Catalytic, Inc. Web Page, http:// www.catalyticinc.com/, April 2005.

[4] Matlab External Interfaces Reference, Version 6, July 2002.

[5] TMS320C28x Optimizing C/C++ Compiler User's Guide, SPRU514, August 2001.

[6] TMS320C2x/C2xx/C5x Optimizing C Compiler User's Guide, SPRU025e, August 1999.

[7] TMS320C6000 Programmer's Guide, SPRU198D, March 2004.
\end{abstract}




\title{
Matlab as a Development Environment for FPGA Design
}

Tejas M. Bhatt, Dennis McCain

Nokia Research Center, Irving, TX

\begin{abstract}
In this paper we discuss an efficient design flow from Matlab ${ }^{\circledR}$ to FPGA. Employing Matlab for algorithm research and as system level language allows efficient transition from algorithm development to implementation. We show that integrating Matlab with HDL design tools such as HDL designer ${ }^{\circledR}$ and Precision-C $($, an efficient design flow, suitable for rapid prototyping, can be obtained. The design flow accelerates process of algorithm development and simplifies testbench formulation and verification process. The overall development time thus can be significantly reduced. We elaborate on the advantages and disadvantages of the design flow. It will be shown that Matlab based design flow generates functional specifications that are useful for RTL development.
\end{abstract}

Keywords: System Design Flows, Rapid Prototyping

\section{REFERENCES}

[1] http://www.mathworks.com/products/fixed/

[2] http://www.catalyticinc.com/deltafx.html

[3] Guo, Y., Xu, G., McCain, D and Cavallaro, J. R. Rapid Scheduling of Efficient FPGA Architectures for NextGeneration HSDPA Wireless System Using Precision C Synthesizer. IEEE International Workshop on Rapid Systems Prototyping, pp. 179-185, San Diego, CA, (June 2003).

[4] 3GPP, Technical Specification, 25.211, v5.5.0, 2003-09.

[5] Zhang, J. Bhatt, T. and Mandyam, G., Efficient Linear Equalization for High Data Rate Downlink CDMA Signaling. 37th IEEE Asilomar Conference on Signals, Systems, and Computers, pp. 141 - 145, vol. 1, Monterey, CA, (Nov. 2003).

[6] http://www.mathworks.com/products/modelsim/ 


\title{
Should Our Power Approach Be Current?
}

Chair: $\quad$ Tim Fox - Deutsche Bank Equity Research

Panelists: $\quad$ David Heacock - Texas Instruments, Inc., Dallas, TX

Ed Huijbregts - Magma Design Automation, Inc., Santa Clara, CA

Vess Johnson - Nascentric, Inc., Austin, TX

Avner Kornfeld - Intel Corp., Santa Clara, CA

Andrew Yang - Apache Design Solutions, Inc., Mountain View, CA

Paul Zuchowski - IBM Corp., Armonk, NY

\begin{abstract}
In the past, power consumption was of little concern to the IC designer. Time-to-market drove the design deadlines, and power consumption was a secondary, if not tertiary, concern. If there were power issues, they could typically be accounted for by tweaking the fabrication process, redesigning after the initial design ship, or even just waiting for the next process change from the fab.

Today power has become one of the sign-off qualifiers prior to fabrication, and the metric for success has changed from performance and area to power consumption in nanometer SoC designs, especially in the huge market for handheld/wireless consumer electronics. Although "power" is often the stated concern, current is the real issue. This fundamental paradigm shift requires changes to both the design flow and the tools used for electrical sign-off.

Keywords: Low-power design, power analysis, leakage current, energy consumption, static power, dynamic power
\end{abstract}




\title{
DTM: Dynamic Tone Mapping for Backlight Scaling
}

\author{
Ali Iranli, Massoud Pedram \\ University of Southern California
}

\begin{abstract}
This paper proposes an approach for pixel transformation of the displayed image to increase the potential energy saving of the backlight scaling method. The proposed approach takes advantage of human visual system characteristics and tries to minimize distortion between the perceived brightness values of the individual pixels in the original image and those of the backlight-scaled image. This is in contrast to previous backlight scaling approaches which simply match the luminance values of the individual pixels in the original and backlight-scaled images. Moreover, the proposed dynamic backlight scaling approach, which is based on tone mapping, is amenable to highly efficient hardware realization because it does not need information about the histogram of the displayed image. Experimental results show that the dynamic tone mapping for backlight scaling method results in about $35 \%$ power saving with an effective distortion rate of $5 \%$ and $55 \%$ power saving for a $20 \%$ distortion rate.
\end{abstract}

Keywords: LCDs, Backlight-scaling, Power Management

\section{References}

[1] J. Williams, “A fourth generation of LCD backlight technology,” Linear Tech. App. Note 65, Nov. 1995.

[2] N. Chang, I. Choi, H. Shim, "DLS: Dynamic Backlight Luminance Scaling of Liquid Crystal Display," IEEE Transactions on Very Large Scale Integration Systems, Vol. 12, No. 8, Aug. 2004, pp.837-846.

[3] W-C. Cheng and M. Pedram, "Power Minimization in a Backlit TFT-LCD by Concurrent Brightness and Contrast Scaling," IEEE Transactions on Consumer Electronics, Vol. 50, No. 1, Feb. 2004, pp. 25-32.

[4] A. Iranli, M.Pedram, "HEBS: Histogram Equalization for Backlight Scaling," Proc. of Design Automation and Test in Europe, Feb. 2005.

[5] H.R. Blackwell, "Contrast thresholds of human eye," Journal of Optical Society of America, No. 36, Vol. 11, Nov. 1946.

[6] S. S. Stevens, J. C. Stevens, "Brightness Function: Effects on Adaptation", Journal of Optical Society of America, vol. 53, No. 3, pp. 375-385, Mar. 1963

[7] J. Tumblin, H. Rushmeier, "Tone reproduction for computer generated images," IEEE Computer Graphics and Applications, Vol, 13, No. 6, Nov. 1993, pp42-48.

[8] J.A.Ferwerda, S.N.Pattanaik, P. Shirley, D.P.Greenberg, "A model of visual adaptation for realistic image synthesis," Proc. SIGGRAPH 1996, ACM SIGGRAPH: Addison Wesley.

[9] G. Ward, "A contrast-based scalefactor for luminance display,” Graphics Gem IV, P. Heckbert Ed., Cambridge.

[10] K.Chiu, M.Herf, P.Shirley, S.Swamy, C.Wang, and K.Zimmerman, "Spatially Non-uniform Scaling Function for High Contrast Images," Proc. Graphics Interface, MAY 1993.

[11] L. Ward, H. Rushmeier, C. Piatko, “A visibility matching tone reproduction operator for high dynamic range scenes," IEEE Transactions on Visualization and Computer Graphics, Vol.3, No. 4, Oct. 1997, pp291-306

[12] S.N.Pattanaik, J.A. Ferwerda, M.D. Fairchild, D. Greenberg, "A multiscale model of adaptation and spatial vision for realistic image display”, Proc. ACM SIGGRAPH, 1998, pp.287-298.

[13] M.Ashikhmin, "A tone mapping algorithm for high contrast images," Proc. of Eurographics Workshop on Rendering, 2002.

[14] P. Choudhury, J. Tumblin, "The trilateral filter for high contrast images and meshes," In Proc. of the Eurographics Symposium on Rendering, 2003, pp.186-196.

[15] F.Durand, J.Dorsey, "Fast bilateral filtering for display of highdynamic-range images," ACM Transactions of Graphics, Vol.21, No.3, 2002, pp.257-266.

[16] Zhou Wang and Alan C. Bovik, “A Universal Image Quality Index,” IEEE Signal Processing Letters, vol. 9, no. 3, Mar. 2002.

[17] A. G. Weber, "The USC-SIPI image database version 5," USCSIPI Report \#315, Oct. 1997. Also http://sipi.usc.edu/services/database/Database.html.

[18] Digital Image Processing, William K. Pratt, Third Edition, John Wiley \& Sons, 2003.

[19] LG Philips, LP064V1 Liquid Crystal Display. 
[20] H. Aoki, "Dynamic characterization of a-Si TFT-LCD pixels," HP Labs 1996 Technical Reports (HPL-9619),Feb. 1996. 


\title{
Application/Architecture Power CoOptimization for Embedded Systems Powered by Renewable Sources
}

\author{
Dexin Li and Pai H. Chou \\ Department of EECS, University of California, Irvine, CA, USA
}

\begin{abstract}
Embedded systems are being built with renewable power sources such as solar cells to replenish the energy of batteries. The renewable power sources have a wide range of efficiency levels that depend on environment parameters and the current drawn from the circuit. Unlike low-power designs whose goal is to minimize energy consumption, systems with renewable power sources should maximize the efficiency of the sources by load matching. To match the wide dynamic range of solar output, it is necessary to exploit multiple power "knobs" simultaneously. This paper combines computation vs. communication trade-offs, algorithm selection, scheduling and dynamic voltage scaling to maximize the dynamic range of the load over time. Experimental results show one to two orders of magnitude performance improvement for a wireless handheld system running image compression applications.
\end{abstract}

Keywords: power management, renewable power source, power utilization, load matching, architectural optimization

\section{REFERENCES}

[1] T. Sterken, K. Baert, R. Puers, and S. Borghs. Power extraction from ambient vibration. In Proc. of WorkshoponSemiconductor Sensors, pages 680-683, November 2002.

[2] U. Pasaogullari and C.Y. Wang. Computational fluid dynamics modeling of solid oxide fuel cells. In Proc. of Solid Oxide Fuel Cells VIII, Eds. S.C. Singhal and M. Dokiya, pages 1403-1412, 2003.

[3] K. Lahiri, S. Dey, and A. Raghunathan. Communication architecture based power management for battery efficient system design. In Proc. of Design Automation Conference, pages 991-996, June 2002.

[4] J. Luo and N. K. Jha. Battery-aware static scheduling for distributed real-time embedded systems. In Proc. of Design Automation Conference, pages 444-449, June 2001.

[5] S. Park and M. B. Srivastava. Dynamic battery state aware approaches for improving battery utilization. In Proc. of Compilers, Architectures and Synthesis for Embedded Systems, pages 225-231, 2002.

[6] D. Bruni, L. Benini, and B. Ricc. System lifetime extension by battery management: an experimental work. In Proc. of Compilers, Architectures and Synthesis for Embedded Systems, pages 232-237, 2002.

[7] D. Rakhmatov, S. Vrudhula, and D.A. Wallach. A model for battery lifetime analysis for organizing applications on a pocket computer. IEEE Transactions on Very Large Scale Integration (VLSI) Systems, 11:1019-1030, December 2003.

[8] W. Yuana, K. Nahrstedta, S. V. Advea, D. L. Jonesb, and R. H. Kravetsa. Design and evaluation of a cross-layer adaptation framework for mobile multimedia systems. In Proc. of the SPIE/ACM Conference on Multimedia Computing and Networking, January 2003.

[9] S. Mohapatra, R. Cornea, N. Dutt, A. Nicolau, and N. Venkatasubramanian. Integrated power management for video streaming to mobile handsets. In Proc. of ACM Multimedia, November 2003.

[10] P. Stanley-Marbell and D. Marculescu. Dynamic fault-tolerance and metrics for battery powered, failure-prone systems. In in Proc. IEEE/ACM Intl. Conference on Computer-Aided Design (ICCAD), November 2003.

[11] N. Taylor and S.Dey. Adaptive image compression for enabling mobile multimedia communicatiodesigon. In Proc. IEEE Intl. Conference on Communications, pages 1925-1929, 2001.

[12] D.G. Lee and S. Dey. Adaptive and energy efficient wavelet image compression for mobile multimedia data services. In Proc. of Conference on Communications, pages 2484-2490, 2002.

[13] D. Li and P.H. Chou. Maximizing efficiency of solar-powered systems by load matching. In Proc. International Symposium on Low Power Electronic Design (ISLPED), pages 162-167, August 2004.

[14] D.L King, J.A. Kratochvil, and W.E. Boyson. Field experience with a new performance characterization procedure for photovoltaic arrays. In Proc. 2nd World Conference and Exhibition on Photovoltaic Solar Energy Conversion, 1998.

[15] M.W. Davis, A. Hunter Fanney, and B.P. Dougherty. Measured versus predicted performance of building integrated photovoltaics. Journal of Solar Energy Engineering-Transactions of the ASME, 125:21-7, 2003. 


\title{
User-perceived Latency Driven Voltage Scaling for Interactive Applications
}

\author{
Le Yan, Lin Zhong, Niraj K. Jha \\ Dept. of Electrical Engineering, Princeton University, Princeton, NJ
}

\begin{abstract}
Power has become a critical concern for battery-driven computing systems, on which many applications that are run are interactive. System-level voltage scaling techniques, such as dynamic voltage scaling (DVS) and adaptive body biasing (ABB), have been shown to reduce energy consumption effectively. Previous works on DVS and ABB exploit low CPU utilization of the processor to drive voltage scaling. This has become inadequate for modern interactive applications involving high CPU usage. In this work, we target computer responsiveness during voltage scaling to exploit more opportunities for energy reduction. Instead of CPU utilization, we use the user-perceived latency, the delay between user input and computer response, to drive voltage scaling. Considering the tradeoff between energy consumption and computer responsiveness during voltage scaling not only reduces energy consumption effectively, but also ensures good computer responsiveness for interactive applications. Experimental results show that for the 70nm technology, during the execution of seven commonly-used interactive applications, the energy consumption of the processor using userperceived latency driven DVS is reduced by an average of $37.3 \%$, and the user-perceived latency by an average of $18.3 \%$, compared to CPU utilization driven DVS. If both DVS and ABB are performed simultaneously based on the user-perceived latency, then the energy consumption is reduced by another $38.9 \%$ compared to when DVS is performed alone, while maintaining a similar computer responsiveness level. We have implemented user-perceived latency driven voltage scaling under Linux with $\mathrm{X}$ Window system. However, the methodology is extensible to other operating systems as well.
\end{abstract}

Keywords: Adaptive body biasing, computer responsiveness, dynamic voltage scaling, power consumption

\section{REFERENCES}

[1] Intel Xscale, http://www.intel.com.

[2] M. Weiser, B. Welch, A. Demers, and S. Shenker, "Scheduling for reduced CPU energy," in Proc. Symp. Operating System Design \& Implementation, Nov. 1994, pp. 13-23.

[3] T. Pering, T. Burd, and R. Brodersen, "The simulation and evaluation of dynamic voltage scaling algorithms," in Proc. Int. Symp. Low Power Electronics \& Design, Aug. 1998, pp. 76-81.

[4] C. H. Kimand K. Roy, "Dynamic VTH scaling scheme for active leakage power reduction," in Proc. Design, Automation \& Test in Europe Conf., Mar. 2002, pp. 163-167.

[5] K. Nose, M. Hirabayashi, H. Kawaguchi, S. Lee, and T. Sakurai, "VTH-hopping scheme for $82 \%$ power saving in low-voltage processors," in Proc. Custom Integrated Circuits Conf., May 2001, pp. 93-96.

[6] M. Miyazaki, J. Kao, and A. Chandrakasan, "A $175 \mathrm{mV}$ multiply-accumulate unit using an adaptive supply voltage and body bias architecture," in Proc. Int. Conf. Solid-State Circuits, Feb. 2002, pp. 58-59.

[7] S. M. Martin, K. Flautner, T. Mudge, and D. Blaauw, "Combined dynamic voltage scaling and adaptive body biasing for lower power microprocessors under dynamic workloads," in Proc. Int. Conf. Computer-Aided Design, Nov. 2002, pp. 721-725.

[8] L. Yan, J. Luo, and N. K. Jha, "Combined dynamic voltage scaling and adaptive body biasing for heterogeneous distributed real-time embedded systems," in Proc. Int. Conf. Computer-Aided Design, Nov. 2003, pp. 357-364.

[9] D. P. Siewiorek, "New frontiers of application design," Commun. ACM, vol. 45, no. 12, pp. 79-82, Dec. 2002.

[10] B. Schneiderman, Designing the User Interface: Strategies for Effective Human-Computer Interaction, 3rd ed. Addison Wesley Longman, 1998.

[11] S. K. Card, T. P. Moran, and A. Newell, The Psychology of Human-computer Interaction. Lawrence Erlbaum Associates, 1983. 
[12] A. Stratakos, High-efficiency Low-voltage DC-DC Conversion for Portable Applications. Ph.D. dissertation, Univ. of California, Berkeley, 1998. 


\title{
System-Level Energy-Efficient Dynamic Task Scheduling
}

Jianli Zhuo, Chaitali Chakrabarti

Department of Electrical Engineering, Arizona State University, Tempe, AZ

\begin{abstract}
Dynamic voltage scaling (DVS) is a well-known low power design technique that reduces the processor energy by slowing down the DVS processor and stretching the task execution time. But in a DVS system consisting of a DVS processor and multiple devices, slowing down the processor increases the device energy consumption and thereby the system-level energy consumption. In this paper, we present dynamic task scheduling algorithms for periodic tasks that minimize the system-level energy (CPU energy + device standby energy). The algorithms use a combination of (i) optimal speed setting, which is the speed that minimizes the system energy for a specific task, and (ii) limited preemption which reduces the numbers of possible preemptions. For the case when the CPU power and device power are comparable, these algorithms achieve up to $43 \%$ energy savings compared to [1], but only up to $12 \%$ over the nonDVS scheduling. If the device power is large compared to the CPU power, we show that DVS should not be employed.

Keywords: Dynamic task scheduling, energy minimization, optimal scaling point, DVS system, real-time
\end{abstract}

\section{REFERENCES}

[1] W. Kim, J. Kim and S. Min, "Preemption-aware dynamic voltage scaling in hard real-time systems," Proc. ISLPED, pp. 393-398, 2004.

[2] F.Yao, A.Demers, and S.Shenker, "A scheduling model for reduced cpu energy," IEEE Annual Foundations of Computer Science, pp. 374-382, 1995.

[3] G. Quan, and X. Hu, "Energy efficient fixed-priority scheduling for real-time systems on variable voltage processors," Proc. DAC, pp. 828-833, 2001.

[4] R. Jejurikar, and R. Gupta, "Leakage aware dynamic voltage scaling for real-time embedded systems," Proc. $D A C$, pp. 275-280, 2004.

[5] Y.Shin, K.Choi, and T.Sakurai, "Power optimization of real-time embedded systems on variable speed processors," Proc. ICCAD, pp. 365-368, 2000.

[6] C.M Krishna and Y.H Lee, "Voltage-clock-scaling adaptive scheduling techniques for low power in hard real time systems," Proc. Real-Time Technology \& Applications Symp., pp. 156-165, 2000.

[7] F. Gruian, "Hard real-time scheduling for low energy using stochastic data and dvs processors," Proc. ISLPED, pp. 46-51, 2001.

[8] D. Shin, J.Kim and S. Lee, "Low-energy intra task voltage scheduling using static timing analysis," Proc. DAC, pp. 438-443, 2001.

[9] W. Kim, J.Kim and S. Min, "A dynamic voltage scaling algorithm for dynamic-priority hard real-time systems using slack time analysis," Proc. DATE., pp. 788-794, 2002.

[10] J.Zhuo and C.Chakrabarti, "An efficient dynamic task scheduling algorithm for battery powered dvs system," Proc.ASP-DAC, pp. 846-849, 2005.

[11] R. Jejurikar, C. Pereira, and R. Gupta, "Dynamic voltage scaling for systemwide energy minimization in realtime embedded systems," Proc. ISLPED, pp. 78-81, 2004.

[12] Intel Corp., http://www.intel.com.

[13] B.Zhai, D.Blaauw, D.Sylvester and K.Flautner, "Theoretical and practival limits of dynamic voltage scaling," Proc. DAC, pp. 868-873, 2004.

[14] Micro Technology, Inc., http://www.micron.com. 
DAC05, pages 632-637

OPERA: Optimization with Ellipsoidal uncertainty for Robust Analog IC design

\author{
Yang Xu ${ }^{1}$, Kan-Lin Hsiung ${ }^{2}$, Xin Li $^{1}$, Ivan Nausieda ${ }^{3}$, Stephen Boyd ${ }^{2}$, Lawrence Pileggi ${ }^{1}$ \\ ${ }^{1}$ Elec. \& Comp. Eng. Dept., Carnegie Mellon University, Pittsburgh, PA \\ ${ }^{2}$ Elec. Eng. Dept., Stanford University, Stanford, CA \\ ${ }^{3}$ Eng. \& Appl. Sci. Div., Harvard University, Cambridge, MA
}

\begin{abstract}
As the design- anufacturing interface becomes increasingly complicated with IC technology scaling, the corresponding process variability poses great challenges for nanoscale analog/RF design. Design optimization based on the enumeration of process corners has been widely used, but can suffer from inefficiency and overdesign. In this paper we propose to formu ate the analog and RF design with variability problem as a special type of robust optimization problem, namely robust geometric programming. The statistical variations in both the process parameters and design variables are captured by a pre-specified confidence ellipsoid. Using such optimization with ellipsoidal uncertainty approach, robust design can be obtained with guaranteed yield bound and lower design cost, and most importantly, the problem size grows linearly with number of uncertain parameters. Numerical examples demonstrate the efficiency and reveal the trade-off between the design cost versus the yield requirement. We will also demonstrate significant improvement in the design cost using this approach compared with corner-enumeration optimization.
\end{abstract}

Keywords: Statistical optimization

\title{
REFERENCES
}

[1] J. Carballo and S. Nassif. Impact of design-manufacturing interface on SoC design methodologies. IEEE Design \& Test of Computers, 2 1(3 ):183-19 1, May-June 2004.

[2] S. D irector, P. Feldmann, and K . Krishna. Statistical integrated circuit design. IEEE JSSC, 28 (3):193 - 202 , March 1993 .

[3] T. Mukherjeee, L. Carley, and R. Rutenbar. Efficient handling of operating range and manufacturing line variations in analog cell synthesis. IEEE TC AD, 19(8):825 - 839, August 2000.

[4] A. Dharchoudhury and S. Kang. Worst-case analysis and optimization of VLSI circuit performance. IEEE TCAD, 14(4):481- 492, April 1995.

[5] P. McNamara, S. Saxena, and C. Guardiani et al. Design for manufacurability characterization and optimization of mixed-signal IP. In Proc . IEEE CICC, pages 271-274, 2001.

[6] M. Hershenson, S. Boyd, and T. H . Lee . Optimal design of a CMOS op-amp via geometric programming . IEEE TCAD, 20(1):1-21, January 2001.

[7] K.-L. Hsiung, S.-J. Kim, and S. Boyd. Tractable approximate robust geometric programming. Revised for publication in Mathematical Programming, April 2005. Available at http://www.stanford.edu/ boyd/rgp.html.

[8] S. Nassif. Design for variability in DSM technologies. In Proc. First Int. Symp. on Quality Electronic Design, pages 451-454, San Jose, California, March 2000.

[9] A. Ben-Tal and A. Nemirovski. Robust optimization: methodology and applications. Mathematical Programming Series B, 92(3):453 - 480, May 2002.

[10] S. Boyd and L. Vandenberghe. Convex Optimization. Cambridge University Press, 2004.

[11] S. Boyd, S.-J. Kim, L. Vandenberghe, and A. Hassibi. A tutorial on geometric programming. Technical report, Electrical Engineering Department, Stanford University, September 2004. Available at www.stanford.edu/ bod/ gp_tutorial.html.

[12] W. Daems, G. Gielen, and W. Sansen. Simulation-based generation of posynomial performance models for the sizing of analog integrated circuits. IEEE TCAD, 22(5 ):517 - 534, May 2003.

[13] M. Hershenson, S. S. Mohan, S. P. Boyd, and T. H. Lee. Optimization of inductor circuits via geometric programming. In Proc. 36th ACM/IEEE Design Automation Conference, pages 994 - 998, June 1999.

[14] M. Romeo, V. Da Costa, and F. Bardou . Broad distribution effects in sums of lognormal random variables. European Physical Journal, pages 513 - 525, May 2003. 
[15] K. Chen, C. Hu, P. Fang, M. R. Lin, and D. L. Wollesen. Predicting CMOS speed with gate oxide and voltage scaling and interconnect loading effects. IEEE Trans. Electron Devices, 44(11):1951-1957, November 1997.

[16 ] K. Chen and C. Hu. Performance and $V_{d d}$ scaling in deep submicrometer CMOS. IEEE JSSC, 33(10):1586 1589, October 1998.

[17 ] A. Hajimiri, S. Limotyrakis, and T. H. Lee. Jitter and phase noise in ring oscillators. IEEE JSSC, 34(6):790 804, June 1999. 


\title{
A Unified Optimization Framework for Equalization Filter Synthesis
}

Jihong Ren and Mark Greenstreet

Dept. of Computer Science, University of British Columbia, Vancouver, BC

\begin{abstract}
We present a novel method for jointly optimizing FIR filters for pre-equalization, decision feedback equalization, and near-end crosstalk cancellation. The unified optimization problem is a linear program, and we describe sparse matrix techniques for its efficient solution. We illustrate our approach with uni- and bi-directional buses using differential signaling in both intra-board and cross-backplane scenarios.
\end{abstract}

Keywords: crosstalk, equalizing filters, linear programming, optimal synthesis

\section{REFERENCES}

[1] International Technology Roadmap for Semiconductors. http://public.itrs.net/Files/2003ITRS/Test2003.pdf, 2003.

[2] G. Balamurugan and N. Shanbhag. Modeling and mitigation of jitter in multiGbps source-synchronous I/O links.

21st Int'l. Conf. on Computer Design, pages 254-260, 2003.

[3] W. Dally and J. Poulton. Transmitter equalization for 4-GBPs signaling. IEEE Micro, 1:48-56, 1997.

[4] W. Dally and J. Poulton. Digital Systems Engineering. Cambridge University Press, 1998.

[5] A. Fiedler, R. Mactaggart, et al. A $1.0625 \mathrm{Gbps}$ transceiver with $2 \mathrm{x}$-oversampling and transmit signal preemphasis. In Proc. of ISSCC97, pages 238-239, 1997.

[6] G.H. Golub and C.F. Van Loan. Matrix Computations, 7th ed. Johns Hopkins, page 51, 1996.

[7] Ron Ho. Personal Communication, 2004

[8] R. Kollipara, G.J. Yeh, et al. Design, Modeling and Characterization of High Speed Backplane Interconnects. High-Performance System Design Conf., 2003.

[9] J. Nocedal and S. Wright. Numerical Optimization, pages 395-417, Springer Press, 1999.

[10] J. Ren and M. Greenstreet. Crosstalk Cancellation for Realistic PCB Buses. In Proc. of the 14th Int. Workshop on Power and Timing Modeling, Optimization and Synthesis (PATMOS), Springer - LNCS, 2004.

[11] J. Ren and M. Greenstreet. A Signal Integrity Test Bed for PCB Buses. In Proc. of the Int'l. Conf. Computer Design, pages 2004.

[12] S. Sidiropoulos and M. Horowitz. A $700-\mathrm{Mb} / \mathrm{s} / \mathrm{pin}$ CMOS signaling interface using current integrating receivers. IEEE J. Solid State Circuits, 32(5):681-690, May 1997.

[13] V. Stojanovic, G. Ginis, and M. Horowitz. Transmit pre-emphasis for high-speed time-division-multiplexed serial-link transceiver. IEEE Trans. on Communications, 38:551-558, 2001.

[14] The Mathworks Inc. http://www.mathworks.com

[15] J.L. Zerbe, C.W. Werner, et al. Equalization and Clock Recovery for a 2.5-10 Gb/s 2-PAM/4-PAM Backplane Transceiver cell. IEEE J. Solid-State Circuits, 38(12):2121-2130, Dec. 2003. 


\title{
Template-Driven Parasitic-Aware Optimization of Analog Integrated Circuit Layouts
}

Sambuddha Bhattacharya, Nuttorn Jangkrajarng and C-J. Richard Shi

Department of Electrical Engineering, University of Washington, Seattle, WA

\begin{abstract}
Layout parasitics have great impact on analog circuit performance. This paper presents an algorithm for explicit parasitic control during layout retargeting of analog integrated circuits. In order to ensure desired circuit performance, bounds on layout parasitics' magnitudes are determined first. Then, graph techniques are coupled with mathematical programming to constrain layout geometry based on these parasitic bounds. The algorithm has been demonstrated to ensure desired circuit performance during technology migration and performance specification changes.

Keywords: Analog Layout Automation, Parasitics, Sensitivity, Optimization

\section{REFERENCES}

[1] B. Razavi, Design of Analog CMOS Integrated Circuits, McGraw Hill, 2001.

[2] K. Lampaert, G. Gielen and W. Sansen, "A performance- driven placement tool for analog integrated circuits", IEEE Jour. Solid State Circuits, vol. 30, pp. 773-780, Jul. 1995.

[3] S. Bhattacharya, N. Jangkrajarng, R. Hartono and C.-J. R. Shi, "Correct-by-construction layout-centric retargeting of large analog designs”, Proc. IEEE/ACM Design Automation Conference, Jun. 2004, pp. $139-144$.

[4] R. Okuda, T. Sato, H. Onodera and K. Tamaru, "An efficient algorithm for layout compaction problem with symmetry constraints", Proc. IEEE/ACM Int. Conference on Computer-Aided-Design, Nov. 1989, pp. $148-151$.

[5] U. Choudhury and A. Sangiovanni-Vincentelli, "Use of performance sensitivities in routing analog circuits", Proc. IEEE Int. Symp. Circuits and Systems, vol.4, May 1990, pp. 348-351.

[6] Mosek manual, http://www.mosek.com/documentation.html.
\end{abstract}




\title{
Multi-level Approach for Integrated Spiral Inductor Optimization
}

\author{
Arthur Nieuwoudt and Yehia Massoud \\ Department of Electrical and Computer Engineering, Rice University, Houston, Texas
}

\begin{abstract}
The efficient optimization of integrated spiral inductors remains a fundamental barrier to the realization of effiective analog and mixed-signal design automation. In this paper, we develop a scalable multi-level optimization methodology for spiral inductors that integrates the exibility of constrained global optimization using Mesh-Adaptive Direct Search (MADS) algorithms with the rapid convergence of local nonlinear convex optimization techniques. Experimental results indicate that our methodology locates optimal spiral inductor geometries with significantly fewer function evaluations than current techniques.
\end{abstract}

Keywords: Spiral Inductor, Inductor Optimization, Analog Synthesis

\section{REFERENCES}

[1] C. Audet and J.E. Dennis. Mesh Adaptive Direct Search Algorithms for Constrained Optimization. Les Journees de l'optimisation, 2004.

[2] M. del Mar Hershenson, S. Mohan, S. Boyd, and T. Lee. Optimization of Inductor Circuits via Geometric Programming. In Proceedings IEEE/ACM Design Automation Conference, 1999 June.

[3] H. Greenhouse. Design of Planar Rectangular Microelectronic Inductors. IEEE Trans. on Parts, Hybrids, and Packaging, pages 101 - 109, June 1974.

[4] D. Melendy and A. Weisshaar. A New Scalable Model for Spiral Inductors on Lossy Silicon Substrate. In 2003 IEEE MTT-S International Microwave Symposium Digest, pages 1007 - 1010, June 2003.

[5] A. Niknejad and R. Meyer. Design, Simulation and Applications of Inductors and Transformers for Si RF ICs. Kluwer Academic Publishers, 2000.

[6] J. Post. Optimizing the Design of Spiral Inductors on Silicon. IEEE Trans. on Circuits and Systems II, $47(1): 15$ 17, Jan. 2000.

[7] J. Sieiro, J. Lopez-Villegas, J. Cabanillas, J. Osorio, and J. Samitier. A Physical Frequency-Dependent Compact Model for RF Integrated Inductors. IEEE Trans. on Microwave Theory and Techniques, pages 384 - 392 , Jan. 2002.

[8] P. Venkataraman. Applied Optimization with Matlab Programming. Wiley, 2002.

[9] C. Wu, C. Tang, and S. Liu. Analysis of On-Chip Spiral Inductors Using the Distributed Capacitance Model. IEEE Journal of Solid-State Circuits, 38(6):1040 - 1044, June 2003.

[10] C. Yue and S. Wong. Physical Modeling of Spiral Inductors on Silicon. IEEE Trans. on Electron Devices, pages 560 - 568, March 2000.

[11] Y. Zhan and S. Sapatneker. Optimization of Integrated Spiral Inductors Using Sequential Quadratic Programming. In Proceedings of the Design, Automation and Test in Europe Conference and Exhibition, January 2004. 


\title{
Statistical Static Timing Analysis: How simple can we get?
}

\author{
Chirayu S. Amin †, Noel Menezes $\neq$, Kip Killpack $\neq$, Florentin Dartuł, \\ Umakanta Choudhuryf, Nagib Hakim $\neq$, Yehea I. Ismail † \\ $\uparrow$ ECE, Northwestern University, Evanston, ILUSA \\ †Intel Corporation, Hillsboro, OR,USA
}

\begin{abstract}
With an increasing trend in the variation of the primary parameters affecting circuit performance, the need for statistical static timing analysis (SSTA) has been firmly established in the last few years. While it is generally accepted that a timing analysis tool should handle parameter variations, the benefits of advanced SSTA algorithms are still questioned by the designer community because of their significant impact on complexity of STA flows. In this paper, we present convincing evidence that a path-based SSTA approach implemented as a post-processing step captures the effect of parameter variations on circuit performance fairly accurately. On a microprocessor block implemented in $90 \mathrm{~nm}$ technology, the error in estimating the standard deviation of the timing margin at the inputs of sequential elements is at most 0.066 FO4 delays, which translates in to only $0.31 \%$ of worst case path delay.
\end{abstract}

Keywords: Statistical Static Timing Analysis (SSTA), Process Variations

\section{References}

[1] H. Chang and S. S. Sapatnekar, "Statistical timing analysis considering spatial correlations using a single Pertlike traversal," ICCAD '03, pp. 621-625.

[2] C. Visweswariah, K. Ravindran, K. Kalafala, S. G. Walker, and S. Narayan, "First-order incremental blockbased statistical timing analysis," $D A C^{\prime}$ '04, pp. 331-336.

[3] J. A. G. Jess et. al., "Statistical timing for parametric yield prediction of digital integrated circuits," $D A C$ '03, pp. 932-937.

[4] A. Devgan and C. Kashyap, "Block-based static timing analysis with uncertainty," ICCAD '03, pp. 607-614.

[5] M. Orshansky and A. Bandyopadhyay, "Fast statistical timing analysis handling arbitrary delay correlations," $D A C^{\prime} 04$, pp. 337-342.

[6] M. Orshansky and K. Keutzer, “A general probabilistic framework for worst case timing analysis," $D A C$ '02, pp. 556-561.

[7] A. Agarwal, V. Zolotov, and D. Blaauw, "Statistical timing analysis using bounds and selective enumeration," IEEE Trans. on CAD, vol. 22, no. 9, pp. 1243-1260, September 2003.

[8] A. Agarwal, D. Blaauw, and V. Zolotov, "Statistical timing analysis for intra-die process variations with spatial correlations," ICCAD '03, pp. 900-907.

[9] F. N. Najm and N. Menezes, "Statistical timing analysis based on a timing yield model," $D A C$ '04, pp. 460-465.

[10] A. Gattiker, S. Nassif, R. Dinakar, and C. Long, "Timing yield estimation from static timing analysis," ISQED '01, pp. 437-442.

[11] S. Nassif, "Delay variability: sources, impact and trends," ISSCC '00, pp. 368-369.

[12] A. Papoulis and S. U. Pillai, Probability, Random Variables, and Stochastic Processes, fourth edition, McGraw Hill, 2002.

[13] J. K. Ousterhout, “A switch-level timing verifier for Digital MOS VLSI," IEEE Trans. on CAD, vol. 4, no. 3, pp. 336-349, July 1985.

[14] N. P. Jouppi, "Timing analysis and performance improvement of MOS VLSI Designs," IEEE Trans. on CAD, vol. CAD-6, no. 4, pp. 650-665, July 1987.

[15] International Technology Roadmap for Semiconductors (ITRS), 2003 edition, Semiconductor Industry Association (http://public.itrs.net/).

[16] T. Sakurai and A. Newton, "Alpha-power law MOSFET model and its application to CMOS inverter delay and other formulas," IEEE JSSC, vol. 25, no. 2, pp. 584-594, April 1990.

[17] S. Samaan, "The Impact of Device Parameter Variations on the Frequency and Performance of VLSI Chips," ICCAD '04. (Also published in ISSCC '04.) 


\title{
Mapping Statistical Process Variations Toward Circuit Performance Variability: An Analytical Modeling Approach
}

\author{
Yu Cao, Lawrence T. Clark \\ Department of EE, ASU, Tempe, AZ
}

\begin{abstract}
A physical yet compact gate delay model is developed integrating short-channel effects and the Alpha-power law based timing model. This analytical approach accurately predicts both nominal delay and delay variability over a wide range of bias conditions, including sub-threshold. Excellent model scalability enables efficient mapping between process variations and delay variability at the circuit level. Based on this model, relative importance of physical effects on delay variability has been identified. While effective channel length variation is the leading source for variability at current $90 \mathrm{~nm}$ node, performance variability is actually more sensitive to threshold variation at the sub-threshold region. Furthermore, this model is applied to investigate the limitation of low power design techniques in the presence of process variations, particularly dual $V_{t h}$ and $L$ biasing. Due to excessive variability under low $V_{D D}$, these techniques become ineffective.
\end{abstract}

Keywords: Process Variations, Delay, Variability

\section{REFERENCES}

[1] International Technology Roadmap for Semiconductors, 2003.

[2] K. A. Bowman, S. G. Duvall, J. D. Meindl, "Impact of die-to-die and within-die parameter fluctuations on the maximum clock frequency distribution for gigascale integration," JSSC, vol. 37, no. 2, pp. 183-190, Feb. 2002.

[3] D. Boning and S. Nassif, "Models of process variations in device and interconnect," Design of HighPerformance Microprocessor Circuits, Chapter 6, pp. 98-115, IEEE Press, 2000.

[4] C. Viswesvariah, "Statistical timing of digital integrated circuits," ISSCC, 2004.

[5] M. Orshansky and K. Keutzer, "A general probabilistic framework for worst-case timing analysis," $D A C$, pp. 556-561, 2002.

[6] H. Chang, S. S. Sapatnekar, "Statistical timing analysis considering spatial correlations using a single PERT-like traversal," ICCAD, pp. 621-625, 2003.

[7] A. Agarwal, D. Blaauw, V. Zolotov, "Statistical timing analysis for intra-die process variations with spatial correlations," ICCAD, pp. 900-907, Nov. 2003.

[8] S. Nassif, N. Hakim, D. Boning, "The care and feeding of your statistical static timer," embedded tutorial, ICCAD, Nov. 2004.

[9] M. Orshansky, J. C. Chen, C. Hu, "Direct sampling methodology for statistical analysis of scaled CMOS technologies," TSM, vol. 12, no. 4, pp. 403-408, Nov. 1999.

[10] T. Sakurai and A. R. Newton, "Alpha-power law MOSFET model and its applications to CMOS inverter delay and other formulas," JSSC, vol. 25, no. 2, pp. 584-594, Apr. 1990.

[11] M. Eisele et al, "The impact of intra-die device parameter variations on path delays and on the design for yield of low voltage digital circuits," TVLSI, vo. 5, no. 4, pp. 360-368, Dec. 1997

[12] BSIM4.2.1 MOSFET Model - User's Manual, 2001.

[13] K. A. Bowman et al., “A physical Alpha-power law MOSFET model,” ISLPED, pp. 218-222, Aug. 1999.

[14] P. Friedberg et al., "Modeling within-die spatial correlation effects for process-design co-optimization," ISQED, Mar. 2005.

[15] P. A. Stolk, F. P. Widdershoven, and D. B. M. Klaassen, "Modeling statistical dopant fluctuations in MOS transistors," TED, vo. 45, no. 9, pp. 1960-1971, Sep. 1998.

[16] J. Tschanz et al., "Adaptive body bias for reducing impacts of die-to-die and within-die parameter variations on microprocessor frequency and leakage,” JSSC, vol. 1, pp. 422-478, Feb. 2002 


\title{
Power Grid Simulation Via Efficient Sampling-Based Sensitivity Analysis and Hierarchical Symbolic Relaxation
}

\author{
Peng $\mathrm{Li}$ \\ Department of Electrical Engineering, Texas A\&M University, College Station, TX, USA
}

\begin{abstract}
On-chip supply networks are playing an increasingly important role for modern nanometer-scale designs. However, the ever growing sizes of power grids make the analysis problem extremely difficult thereby introducing severe challenges in design and optimization. The inherent analysis complexity calls for innovations in simulation techniques that must provide appropriate accuracy, efficiency as well as the tradeoff thereof to aid design verification and optimization. In this paper, we first present a sampling-based sensitivity analysis by employing the notation of importance sampling in a Monte Carlo based circuit simulation framework. This technique allows the extraction of multiparameter sensitivities for the node voltages of interest in the same Monte Carlo runs that are used for computing the nominal voltage values. For more efficient nonstructured whole-grid solution approaches, we further introduce a new direct solution method by embedding symbolic relaxation steps in a hierarchical fashion. As a direct method, the proposed hierarchical symbolic relaxation is suitable to both dc and transient analyses. Circuit examples are included to demonstrate the efficacy of the proposed techniques.
\end{abstract}

Keywords: Power grids, sensitivity and hierarchical analysis

\section{REFERENCES}

[1] S. Nassif and J. Kozhaya, "Fast power grid simulation," IEEE/ACM DAC, 2000.

[2] J. Kozhaya, S. Nassif and F. Najm, "A multi-grid like technique for power grid analysis," IEEE Trans. CAD, vol. 21, no. 10, Oct. 2002.

[3] M. Zhao, R. Panda, S. Sapatnekar and D. Blaauw, "Hierarchical analysis of power distribution networks," IEEE Trans. on CAD, vol. 21, no. 2, Feb. 2002.

[4] H. Su, E. Acar and S. Nassif, "Power grid reduction based on algebraic multigrid principles", Proc. IEEE/ACM $D A C, 2003$.

[5] H. Qian, S. Nassif and S. Sapatnekar, "Random walks in supply network," Proc. of IEEE/ACM DAC, 2003.

[6] R. Rubinstein,"Simulation and Monte Carlo Method," John Wiley \& Sons, 1981.

[7] H. Qian and S. Sapatnekar, "Hierarchical random-walk algorithms for power grid analysis," Proc. of IEEE/ACM $A S P-D A C, 2004$.

[8] E. Chiprout, "Fast flip-chip power grid analysis via locality and grid shells," IEEE/ACM ICCAD, 2004.

[9] Y. Coz and R. Iverson, "A stochastic algorithm for high speed capacitance extraction in integrated circuits," Solid State Electronics, vol. 35, no. 7, pp. 1005-1012, 1992.

[10] X. Tan, C. Shi, D. Lungeanu, J. Lee and L. Yuan, "Reliability-constrained area optimization of VLSI power/ground networks via sequence of linear programmings," Proc. of IEEE/ACM DAC, 1999.

[11] S. Director and R. Rohrer, "The generalized adjoint network and network sensitivities," IEEE Trans. Circ. Theory, vol. 16, no. 8, pp. 318-323, Aug 1969.

[12] S. Zhao, K. Roy and C.-K. Koh, "Decoupling capacitance allocation for power supply noise suppression," Proc. of ISPD, 2001.

[13] H. Zheng, L. Pileggi and B. Krauter "Electrical modeling of integrated-package power and ground distributions," IEEE Design \& Test of Computer, vol. 20, issue 3, May-June 2003.

[14] H. Su, S. Sapatnekar and S. Nassif, "Optimal decoupling capacitor sizing and placement for standard-cell layout designs," IEEE Trans. on CAD, vol. 22, no. 4, April 2003.

[15] H. Chen and D. Ling, "Power supply noise analysis methodology for deepsubmicron VLSI chip designs," IEEE/ACM DAC, 1997.

[16] T. Hesterberg, "Advances in importance sampling," Ph.D. Dissertation, Dept. of Statistics, Standford University, 1988.

[17] M. Iosifescu, "Finite Markov processes and their applications," John Wiley \& Sons, 1980. 


\section{Formal Verification - Is It Real Enough?}

Yaron Wolfsthal*, Rebecca M. Gott**

*IBM Haifa Research Laboratory, ISRAEL

**IBM Systems and Technology Group, Poughkeepsie, NY, USA

Keywords: Formal Verification, Functional Verification

\section{REFERENCES}

[1] Ben-David, S., Eisner, C., Geist, D., and Wolfsthal, Y. Model Checking in IBM, Formal Methods in System Design 22, 2 (2003), 101-108.

[2] See pertinent experience reported at the RuleBase homepage http://www.haifa.il.ibm.com/projects/verification/ RB_Homepage

[3] Ginzburg, Y., Using Sugar at the IBM Haifa Design Labs, PSL/Sugar Consortium Meeting at DAC'03, http://www.pslsugar.org/papers/ABV-in-IBM-Haifa.pdf 
Can We Really Do Without the Support of Formal Methods in the Verification of Large Designs?

Umberto Rossi

STMicroelectronics, Agrate Brianza - Italy

General Terms: verification, reliability 


\title{
Streamline Verification Process with Formal Property Verification to Meet Highly Compressed Design Cycle
}

\author{
Prosenjit Chatterjee \\ NVIDIA Corporation, Santa Clara, California
}

\author{
ABSTRACT \\ In this paper, I describe a methodology and tool flow for using formal verification effectively to \\ reduce the verification burden in large custom ASIC designs. \\ Keywords: Formal verification

\section{REFERENCES} \\ [1] Malachowsky, C., "When 10M Gates Just Isn't Enough: The GPU Challenge", DAC, 2002. \\ [2] Smith, D., "NVIDIA: Scaling metholodogy", Proceedings of EDP, 2002. \\ [3] Magellan product description web site, http://www.synopsys.com/products/magellan/magellan.html, 2005. \\ [4] Ibid. \\ [5] Ip, N., and Foster, H., "Design Illumination". DesignCon 2005. \\ [6] Jasper Design Automation, "JasperGold 3.1 Reference Manual", http://www.jasper-da.com/, 2005.
}




\title{
TCAM Enabled On-Chip Logic Minimization
}

Seraj Ahmad, Rabi Mahapatra

Department of Computer Science, Texas A \& M University, College Station, Texas

\begin{abstract}
This paper presents an efficient hardware architecture of an on-chip logic minimization coprocessor. The proposed architecture employs TCAM cells to provide fastest and memory efficient implementation suitable for emerging on-chip minimization applications. The paper presents a detailed design of the on-chip minimizer and shows that it requires very little hardware resources to achieve acceptable quality of minimization. An incremental insertion and bulk deletion is achieved in $0.25 \mu$ s and $3.8 \mathrm{~ms}$ respectively and a compaction of 100000 entries in $25 \mathrm{~ms}$ using just 300 TCAM entries.
\end{abstract}

Keywords: TCAM, Logic Minimization,On-Chip

\section{REFERENCES}

[1] S. Ahmad and R. Mahapatra. m-Trie - A Fast and Efficient Approach to On-Chip Logic Minimization. In Proc. Intl. Conf. on Computer Aided Design. IEEE, November 2004.

[2] S. Choi, K. Sohn, M.-W. Lee, S. Kim, H.-M. Choi, D. Kim, U.-R. Cho, H.-G. Byun, Y.-S. Shin, and H.-J. Yoo. A $0.7 \mathrm{fJ} / \mathrm{bit} / \mathrm{search}, 2.2 \mathrm{~ns}$ search time hybrid type TCAM architecture. In Solid-State Circuits Conference, 2004. Digest of Technical Papers, volume 1, pages 498-542, Feb 2004.

[3] J. Cong and J. Peck. On Acceleration of Logic Synthesis Algorithms using FPGA-based Reconfigurable Coprocessors. Technical Report TR-970010, Computer Science Department, University of California, Los Angeles, CA, 1997.

[4] J. Cong and J. Peck. On Acceleration of the Check Tautology Logic Synthesis Algorithm using an FPGA-based Reconfigurable Coprocessor. In Proceedings of the 5th IEEE Symposium on FPGA-Based Custom Computing Machines (FCCM '97), page 246. IEEE Computer Society, 1997.

[5] B. Gamache, Z. Pfeffer, and S. P. Khatri. A Fast Ternary CAM Design for IP Networking Applications. In 12th International Conference on Computer Communications and Networks (IC3N-03), Dallas, TX, volume 1, pages 498-542, October 2003.

[6] Intrinsyc. Cerfcube 255 with Embedded Linux. http://www.intrinsyc.com/products/cerfcube.

[7] H. Liu. Routing Table Compaction in Ternary-CAM. IEEE, Micro, 15(5):58-64, Jan/Feb 2002.

[8] R. Lysecky and F. Vahid. A Codesigned On-Chip Logic Minimizer. In Proc. CODES+ISSS, pages 109-113. ACM, October 2003.

[9] R. Lysecky and F. Vahid. On-chip logic minimization. In Proc. Design Automation Conference, pages 334-337. ACM, June 2003.

[10] H. Noda, K. Inoue, H. Mattausch, T. Koide, and K. Arimoto. A Cost-Efficient Dynamic Ternary CAM in 130 nm CMOS Technology with Planar Complementary Capacitors and TSR Architecture. In Symposium on VLSI Circuits Digest of Technical Papers, pages 83-84, 2003. 


\title{
Hardware Speech Recognition for User Interfaces in Low Cost, Low Power Devices
}

Sergiu Nedevschi, Rabin K. Patra, Eric A. Brewer

Department of Electrical Engineering and Computer Science, University of California at Berkeley

\begin{abstract}
We propose a system architecture for real-time hardware speech recognition on low-cost, powerconstrained devices. The system is intended to support real-time speech-based user interfaces as part of an effort to bring Information and Communication Technologies (ICTs) to underdeveloped regions of the world. Our system architecture exploits a shared infrastructure model. The computationally intensive task of speech model training and retraining is performed offine by shared servers, while the actual recognition of speech is conducted on low-cost handheld devices using custom hardware. The recognizer is extremely exible and can support multiple languages or dialects with speaker-independent recognition.Dynamic loading of speech models is used for changing language grammar and retraining, while reprogramming is used to support evolution of recognition algorithms. The focus on small sets of words (at one time) reduces the complexity, cost and power consumption. We design the speech decoder, the central component of the recognizer, and we validate it via a prototype FPGA implementation. We then use ASIC synthesis to estimate power and size for the design. Our evaluations demonstrate an order of magnitude improvement in power compared with optimized recognition software running on a low-power embedded general-purpose processor of the same technology and of similar capabilities. The synthesis also estimates the area of the design to be about $2.5 \mathrm{~mm} 2$, showing potential for lower cost. In designing and testing our recognizer we use datasets in both English and Tamil languages.
\end{abstract}

Keywords: Speech recognition, low power, ASIC, tamil, TIER

\section{REFERENCES}

[1] A. Chandrakasan and S. Sheng and R. Brodersen. Low-Power CMOS Digital Design. JSSC, V27, N4, April 1992, pp 473-484.

[2] Amit Sinha, Anantha Chandrakasan. JouleTrack: A Web Based Tool for Software Energy Profiling. DAC, 2001.

[3] ATMEL. ATMEL ATC18 64K x 32-bit Low-power Flash. 2002. http://www.atmel.com.

[4] Binu Mathew and Al Davis and Zhen Fang. A Low-power Accelerator for the SPHINX 3 Speech Recognition System. In International Conference on Compilers, Architectures and Synthesis for Embedded Systems, pages 210219. ACM Press, 2003.

[5] CMU Speech. List of Speech Recognition Hardware Products. 2002. http://www.speech.cs.cmu.edu/ comp.speech/Section6/Q6.5.html.

[6] Danesh Tavana, Bob Colwell, Bill Harris, Barry K. Britton, Bob Brodersen, Chris Rowen. Future Systems-onChip: Software or Hardware Design? DAC Panel Discussion, 2000.

[7] Hsiao-Wuen Hon. A Survey of Hardware Architectures Designed for Speech Recognition. Technical Report CMU-CS-91-169, 1991.

[8] Intel Corporation. Intel PXA 26x Specifications. http://www.intel.com/design/pca/prodbref/251671.htm.

[9] Intel Corporation. Intel SA-1100 Specifications. http: //www.intel.com/design/pca/applicationsprocessors/ 1110_brf.htm.

[10] J. Adam Butts and Gurindar S.Sohi. A Static Power Model for Architects . Proc.of the Intl. Symp. On Micro architecture, 2000.

[11] J.A.Pineiro, M.D.Ercegovac, and J.D.Bruguera. Analysis of the Tradeoffs for the Implementation of a HighRadix Logarithm. IEEE International Conference on Computer Design: VLSI in Computers and Processors, 2002.

[12] Rajeev Krishna, Scott Mahlke, and Todd Austin. Architectural optimizations for low-power, real-time speech recognition. In International Conference on Compilers, Architectures and Synthesis for Embedded Systems, pages 220-231. ACM Press, 2003. 
[13] M. Borgatti, L. Cali, G. De Sandre. A 1GOPS Reconfigurable Signal Processing IC with Embedded FPGA and 3-port 1.2GB/s Flash Memory Subsystem. IEEE International Solid-State Circuits Conference, San Francisco, CA, USA, 2003.

[14] Mentor Graphics. Modelsim: HDL Simulator. http://www.model.com.

[15] Micro Device. BSI Very Low Power CMOS SRAM. www.micro-device.co.jp/ bs62lv256.pdf.

[16] M. Ravishankar. Parallel Implementation of Fast Beam Search for Speaker-Independent Continuous Speech Recognition. Computer Science and Automation, IISc, India , 1993.

[17] Semiconductor Industry Association. International Technology Roadmap for Semiconductors. http://public.itrs.net.

[18] Sensory Technologies. Sensory Speech Products. http://www.sensoryinc.com/.

[19] S.J. Melnikoff, S.F. Quigley, M.J. Russell. Speech recognition on an FPGA using Discrete and Continuous Hidden Markov models. In International Conference on Field Programmable Logic and Applications, pages 202211, 2002.

[20] Speech Vision and Robotics Group,Cambridge University Engineering Department . HTK Tool Kit. http://htk.eng.cam.ac.uk/.

[21] T. S. Anantharaman and R. Bisiani. A Hardware Accelerator for Speech Recognition Algorithms. ISCA, pages 216-223, 1986.

[22] UC Berkeley. The TIER Project. http://tier.cs.berkeley.edu.

[23] United Nations. Unesco Institute of Statistics. http://www.uis.unesco.org.

[24] University of Wisconsin-Madison. Simpescalar Architectural Research Tool Set. http://www.cs.wisc.edu / mscalar/simplescalar.html.

[25] Young. SJ and R. NH and T. JHS. Token Passing: A Simple Conceptual Model for Connected Speech Recognition Systems. Tech Report CUED/F-INFENG/TR38, Cambridge University, 1989. 


\title{
Improving Java Virtual Machine Reliability for Memory-Constrained Embedded Systems
}

Guangyu Chen and Mahmut Kandemir

\author{
Computer Science and Engineering Department, The Pennsylvania State University
}

University Park, PA, USA

\begin{abstract}
Dual-execution/checkpointing based transient error tolerance techniques have been widely used in the high-end mission critical systems. These techniques, however, are not very attractive for cost-sensitive embedded systems because they require extra resources (e.g., large memory, special hardware, etc), and thus increase overall cost of the system. In this paper, we propose a transient error tolerant Java Virtual Machine (JVM) implementation for embedded systems. Our JVM uses dual-execution and checkpointing to detect and recover from transient errors. However, our technique does not require any special hardware support (except for the memory page protection mechanism, which is commonly available in modern embedded processors), and the memory space overhead it incurs is not excessive. Therefore, it is suitable for memoryconstrained embedded systems. We implemented our approach and performed experiments with seven embedded Java applications.
\end{abstract}

Keywords: Java Virtual Machine, dual execution, transient error

\section{REFERENCES}

[1] J2ME, CLDC 1.0.4 reference implementation. http://wwws.sun.com/software/communitysource/j2me/.

[2] J2ME technology for creating mobile devices (white paper). http://java.sun.com/products/clde/wp/KVMwp.pdf.

[3] Us mobile devices to 2006: A land of opportunity. Based on an extensive research program conducted by Datamonitor on mobile device markets in the US.

[4] J. F. Aiegler. Terrestrial cosmic rays. IBM Journal of Research and Development, 40(1), 1996.

[5] D. W. Caldwell and D. A. Rennels. A minimalist fault-tolerant microcontroller design for embedded spacecraft computing. Journal of Supercomputing, 16(1-2), 2000.

[6] D. Chen, A. Messer, P. Bernadat, G. Fu, Z. Dimitrijevic, D. J. F. Lie, D. Mannaru, A. Riska, and D. Milojicic. JVM susceptibility to memory errors. In the 1st USENIX Sysmposium on Java Virtual Machine Research and Technology, 1999.

[7] G. Chen, M. Kandemir, N. Vijaykrishnan, A. Sivasubramaniam, and M. J. Irwin. Analyzing object error behavior in embedded JVM environments. In the IEEE/ACM/IFIP International Conference on Hardware/Software Codesign and System Synthesis, 2004.

[8] B. Cmelik and D. Keppel. Shade: A fast instruction-set simulator for execution profiling. ACM SIGMETRICS Performance Evaluation Review, 22(1), 1994.

[9] M. Gomaa, C. Scarbrough, T. N. Vijaykumar, and I. Pomeranz. Transient-fault recovery for chip multiprocessors. SIGARCH Comput. Archit. News, 31(2), 2003.

[10] J. Gosling, B. Joy, G. Steele, and G. Bracha. Java Language Specification, 2nd Edition. Addison Wesley Professional, 2000.

[11] J. Ray, J. C. Hoe, and B. Falsafi. Dual use of superscalar datapath for transient-fault detection and recovery. In Proceedings of ACM/IEEE international symposium on Microarchitecture. IEEE Computer Society, 2001.

[12] S. K. Reinhardt and S. S. Mukherjee. Transient fault detection via simultaneous multithreading. In ISCA, 2000.

[13] M. Satyanarayanan, H. H. Mashburn, P. Kumar, D. C. Steere, and J. J. Kistler. Lightweight recoverable virtual memory. ACM Trans. Comput. Syst., 12(1), 1994.

[14] R. N. Williams. A painless guide to crc error detection algorithms. ftp.adelaide.edu.au/pub/rocksoft/crc v3.txt. 


\title{
Frequency-Based Code Placement for Embedded Multiprocessors
}

Corey Goldfeder

Columbia University, New York, New York

\begin{abstract}
Multiprocessor embedded systems often have processor-local caches and a shared memory. If the system's code is available at design time we can maximize cache hits by rearranging code in memory so that frequently executed tasks reside in reserved areas of the caches and are not overwritten by less frequent tasks.

Keywords: Embedded Systems, Multiprocessors, Caching, Memory, Code Placement, Frequent Code
\end{abstract}

\section{REFERENCES}

[1] Parameswaran, S. "Code placement in hardware/software co-synthesis to improve performance and reduce cost." In Proceedings of the Conference on Design, Automation and Test in Europe. pages 626-632, 2001

[2] Parameswaran, S. "I-CoPES: fast instruction code placement for embedded systems to improve performance and energy efficiency." In Proceedings of the 2001 IEEE/ACM international conference on Computer-aided design. pages 635-641, 2001

[3] Yanbing, L. and W. Wolf. "A Task-Level Hierarchical Memory Model for System Synthesis of Multiprocessors." In Proceedings of the 34th annual conference on Design automation - Volume 00. pages 153-156, 1997.

[4] Tomiyama, H. and H. Yasuura. "Size-Constrained Code Placement for Cache Miss Rate Reduction." In Proceedings of the 9th International Symposium on System Synthesis. pages 96-104, 1996.

[5] McFarling, S. "Program Optimization for Instruction Caches." In Proceedings of 3rd International Conference on Architectural Support for Programming Languages and Operating Systems, pages 183-191, 1989.

[6] Hennessy, J.L. and D.A. Patterson. Computer Architecture: A Quantitative Approach. Morgan Kaufmann Publishers, Inc. 2nd edition, 1996.

[7] Corman, T., et. al. Introduction to Algorithms. MIT Press, 2001.

[8] Tannenbaum, A. Structured Computer Architecture. Prentice Hall, Inc. 4th edition, 2001 


\title{
Power Emulation: A New Paradigm for Power Estimation \\ Joel Coburn, Srivaths Ravi, and Anand Raghunathan \\ NEC Laboratories America, Princeton, NJ
}

\begin{abstract}
In this work, we propose a new paradigm called power emulation, which exploits hardware acceleration to drastically speedup power estimation. Power emulation is based on the observation that most power estimation tools typically perform the following sequence of operations: simulating the circuit to obtain value traces or statistics for the inputs of its constituent components, evaluating power models for each circuit component based on the input values seen during simulation, and aggregating the power consumption of individual components to compute the circuit's power consumption. We further recognize that the steps involved in power estimation (power model evaluation, aggregation) can themselves be thought of as synthesizable functions and implemented as hardware circuits. Thus, any given design can be enhanced by adding to it .power estimation hardware., and the resulting power model enhanced circuit can be mapped onto a hardware prototyping platform. While drastic speedups in power estimation (orders of magnitude) are possible using this approach, a significant challenge arises due to the increase in circuit size as a result of adding power estimation hardware. We propose a systematic methodology to reduce the size of the power model enhanced circuit through a suite of techniques, including power model reuse across different circuit components, regulating the granularity of components for power modeling, exploiting inter-component power correlations, resource sharing for power model computations, and the use of block memories for efficient storage within power models. We demonstrate the benefits of the proposed power emulation paradigm by applying it to register-transfer level (RTL) power estimation for industrial designs, resulting in speedups from around $10 \mathrm{X}$ to over $500 \mathrm{X}$ compared to state-of-the-art commercial power estimation tools.
\end{abstract}

Keywords: Power Estimation, Emulation, Design, Design Methodologies, Macromodels, FPGA, Hardware Acceleration, Register-Transfer Level, Simulation

\section{REFERENCES}

[1] "International Technology Roadmap for Semiconductors, 2001 Edition". http://public.itrs.net/Files/2001ITRS/ Home.htm.

[2] "Galaxy Power Products, Synopsys Inc" (http://www.synopsys.com)".

[3] "PowerTheater, Sequence Design Inc. (http://www.sequencedesign.com).”.

[4] "PowerChecker, BullDAST s.r.l. (http://www.bulldast.com).".

[5] "ORINOCO, ChipVision Design Systems. (http://www.chipvision.com).".

[6] S. Ravi, A. Raghunathan, and S. Chakradhar, "Efficient RTL power estimation for large designs," in Proc. Int. Conf. VLSI Design, Jan. 2003.

[7] J. Rabaey and M. Pedram (Editors), Low Power Design Methodologies. Kluwer Academic Publishers, Norwell, MA, 1996.

[8] E. Macii, M. Pedram, and F. Somenzi, "High-level power modeling, estimation, and optimization," in Proc. Design Automation Conf., pp. 504-511, June 1997.

[9] A. Raghunathan, N. K. Jha, and S. Dey, High-level Power Analysis and Optimization. Kluwer Academic Publishers, Norwell, MA, 1998.

[10] D. Marculescu, R. Marculescu, and M. Pedram, "Information theoretic measures for energy consumption at the register-transfer level," in Proc. Int. Symp. Low Power Design, pp. 81-86, Apr. 1995.

[11] M. Nemani and F. Najm, "High-level area and power estimation for VLSI circuits," IEEE Trans. ComputerAided Design, vol. 18, pp. 697-713, June 1999.

[12] P. Landman and J. M. Rabaey, "Architectural power analysis: The dual bit type method," IEEE Trans. VLSI Systems, vol. 3, pp. 173-187, June 1995. 
[13] L. Benini, A. Bogliolo, M. Favalli, and G. De Micheli, "Regression models for behavioral power estimation," in Proc. Int. Wkshp. Power \& Timing Modeling, Optimization, and Simulation, 1996.

[14] Q. Wu and Q. Qiu and M. Pedram and C. -S. Ding, "Cycle-accurate macro-models for RT-level power analysis," IEEE Trans. VLSI Systems, vol. 6, pp. 520-528, Dec. 1998.

[15] R. P. Llopis and F. Goossens, "The Petrol approach to high-level power estimation," in Proc. Int. Symp. Low Power Electronics \& Design, pp. 130-132, Aug. 1998.

[16] R. Mehra and J. Rabaey, "Behavioral level power estimation and exploration," in Proc. Int. Wkshp. Low Power Design, pp. 197-202, Apr. 1994.

[17] F. Ferrandi, F. Fummi, E. Macii, M. Poncina, and D. Sciuto, "Power estimation of behavioral descriptions," in Proc. Design Automation \& Test Europe (DATE) Conf., pp. 762-766, Feb. 1998.

[18] L. Kruse, E. Schmidt, G. jochens, A. Stammermann, A. Schulz, E. Macii, and W. Nebel, "Estimation of lower and upper bounds on the power consumption from scheduled data flow graphs," IEEE Trans. VLSI Systems, vol. 9, pp. 3-14, Feb. 2001.

[19] V. Tiwari, S. Malik, and A. Wolfe, "Power analysis of embedded software: A first step towards software power minimization," IEEE Trans VLSI Systems, vol. 2, pp. 437-445, Dec. 1994.

[20] M. Kamble and K. Ghose, "Analytical energy dissipation models for low power caches," in Proc. Int. Symp. Low Power Electronics \& Design, pp. 143-148, Aug. 1997.

[21] M. Chinosi, R. Zafalon, and C. Guardiani, "Automatic characterization and modeling of power consumption in static RAMs," in Proc. Int. Symp. Low Power Electronics \& Design, pp. 112-114, Aug. 1998.

[22] T. D. Givargis, F. Vahid, and J. Henkel, "Evaluating Power Consumption of Parameterized Cache and Bus Architectures in System-on-a-Chip Designs," IEEE Trans. VLSI Systems, vol. 9, pp. 500-508, Aug. 2001.

[23] K. Lahiri and A. Raghunathan, "Power Analysis of System-Level On-Chip Communication Architectures," in Proc. Int. Conf. Hardware/Software Codesign and System Synthesis (CODES+ISSS), pp. 236-241, Sept. 2004.

[24] R. Burch, F. N. Najm, P. Yang, and T. Trick, "A Monte Carlo approach for power estimation," IEEE Trans. VLSI Systems, vol. 1, pp. 63-71, Mar. 1993.

[25] M. Chinosi, R. Zafalon, and C. Guardiani, "Parallel mixed-level power simulation based on spatiotemporal circuit partitioning," in Proc. Design Automation Conf., pp. 562-567, June 1999.

[26] J. Coburn, S. Ravi, and A. Raghunathan, "Hardware Accelerated Power Estimation," in Proc. Design Automation \& Test Europe (DATE) Conf., pp. 528-529, Mar.

[27] "Synplify Pro, Synplicity Inc. (http://www.synplicity.com).".

[28] "Virtex-II FPGA and tools, Xilinx Inc. (http://www.xilinx.com).".

[29] G. G. Roussas, A Course in Mathematical Statistics, Second Edition. Academic Press, London, UK, 1997.

[30] A. K. Jain and R. C. Dubes, Algorithms for Clustering Data. Prentice Hall, Englewood Cliffs, NJ, 1988.

[31] "Using AMPL/MINOS (http://www.ampl.com/BOOKLETS/ampl-minos.pdf).”.

[32] B. Kernighan and S. Lin, "An Efficient Heuristic Procedure for Partitioning Graphs," The Bell System Tech J., vol. 49, pp. 291.307, Feb. 1970.

[33] K. Wakabayashi, C-Based High-Level Synthesis System, .CYBER.-Design Experience-, vol. 41, pp. 264-268. July 2000 .

[34] CB130 Family 0.13um CMOS CBIC http://www.necel.com/cbic/en/cb130/cb130.html.NEC Electronics, Inc., Sept. 2000 


\title{
Implementing Low-Power Configurable Processors - Practical Options and Tradeoffs
}

John Wei and Chris Rowen

Tensilica, Inc., Santa Clara, CA

\begin{abstract}
Configurable processors enable dramatic gains in energy efficiency, relative to traditional fixed instruction-set processors. This energy advantage comes from three improvements. First, configuration of the instruction set permits a much closer fit of the processor to the target applications, reducing the number of execution cycles required. Second, configuring the processor removes unneeded features, reducing power and area overhead. Third, automatic processor generation tools enable logic optimization, signal switching reductions, and seamless mapping into low-voltage circuits and processes, for very low-power operation. The first improvement has been well-studied. Analysis of the second and third improvements requires detailed circuit and layout experiments, which is the primary focus of this paper.
\end{abstract}

Starting from a range of existing available power saving options, this work explores the tradeoff and analyzes the results: the design priority tradeoff, the process technology impact, and implementing low-power configurable processor using commercial scaled-VDD cell libraries compatible with mainstream SOC practices. These real processor designs can achieve power dissipation approaching $20 \mu \mathrm{W} / \mathrm{MHz}$ at $0.8 \mathrm{~V}$ and close to $10 \mu \mathrm{W} / \mathrm{MHz}$ at $0.6 \mathrm{~V}$, using production 0.13 um libraries. Finally, this work quantifies the dramatic process, voltage and temperature dependence in post-layout leakage power for small processor designs.

Keywords: Configurable embedded processor, SOC (system on chip), PVT (process, voltage, temperature), Low-power, Leakage Power, Dynamic Power, Dynamic power efficiency, Scaled VDD

\section{References}

[1] Rowen, C. Engineering the Complex SOC - Fast, Flexible Design with Configurable Processors. Prentice Hall, Upper Saddle River, NJ, 2004, Chapters $7 \& 8$.

[2] System Drivers, International Technology Roadmap for Semiconductors, 2003, 10-11. http://public.itrs.net/.

[3] Artisan Components, Inc. http://www.artisan.com/promo/metro.html

[4] Virtual Silicon Technology, Inc. http://www.virtualsilicon. com/view_press_release.cfm?prid=83

[5] Peters, E., Taglieri, G., and Vemury, L. Low Power Synthesis Flow For a Configurable Core. SNUG (Synopsys Users Group) Boston 2000.

[6] Hillman, D., and Wei, J. Implementing Power Management IP. SNUG (Synopsys Users Group) Boston 2004.

[7] EEMBC press release. EEMBC Developing Standardized Methodology to Measure Energy Consumption. Nov.9, 2004. http://www.eembc.hotdesk.com 


\title{
Low Power Network Processor Design Using Clock Gating
}

Yan Luo, Jia Yu, Jun Yang, Laxmi Bhuyan

University of California Riverside, Riverside, CA

\begin{abstract}
Network processors (NPs) have emerged as successful platforms to providing both high performance and flexibility in building powerful routers. Typical NPs incorporate multiprocessing and multi-threading to achieve maximum parallel processing capabilities. We observed that under low incoming traffic rates, most processing elements (PEs) in NPs are nearly idle and yet still consume dynamic power. This paper develops a low power technique to reduce the activities of PEs according to the varying traffic volume. We propose to monitor the average number of idle threads in a time window, and gate off the clock network of unused PEs when a subset of PEs is enough to handle the network traffic. We show that our technique brings significant reduction in power consumption (up to $30 \%$ ) of NPs with no packet loss and little impact to the overall throughput.
\end{abstract}

Keywords: Network Processors, Low Power

\section{REFERENCES}

[1] D. Brooks, V. Tiwari, M. Martonosi, "Wattch: a framework for architectural-level power analysis and optimizations," ISCA-27, pp. 83-94, 2000.

[2] D. E. Duarte, N. Vijaykrishnan, M. J. Irwin, "A Clock Power Model to Evaluate Impact of Architectural and Technology Optimizations," IEEE TVLSI, pp. 844-855, Vol. 10, Iss. 6, 2002.

[3] M. Franklin and Tilman Wolf, "Power Considerations in Network Processor Design," Workshop on Network Processors - NP2, in conjunction with HPCA9, pp. 10-22, 2003.

[4] S. Kaxiras and G. Keramindas, "IPStash: a Power-Efficient Memory Architecture for IP-lookup," MICRO-36, pp. 361-372, 2003.

[5] H. Li, S. Bhunia, Y. Chen, T.N. Vijaykumar, K. Roy, "Deterministic Clock Gating for Microprocessor Power Reduction," HPCA-9, pp. 113-122, 2003.

[6] Y. Luo, J. Yang, L. Bhuyan, L. Zhao, "NePSim: A Network Processor Simulator with Power Evaluation Framework," IEEE Micro Special Issue on Network Processors for Future High-End Systems and Applications, Sept/Oct 2004.

[7] A. Mallik and G. Memik, "A Case for Clumsy Packet Processors," MICRO-37, pp. 147-156, 2004.

[8] M. D. Powell, S. Yang, B. Falsafi, K. Roy, T. N. Vijaykumar, "Gated-Vdd: A Circuit Technique to Reduce Leakage in Deep-Submicron Cache Memories," ISLPED, pp. 90-95, 2000. [9] The NLANR Measurement and Network Analysis, http://www.nlanr.net/

[10] Intel Corporation, "IXP1200 Network Processor Family Hardware Reference Manual," http://developer.intel.com/design/netwrok/ixa.html, 2001.

[11] Intel IXP2XXX Product Line of Network Processors, http://www.intel.com/design/network/products/ npfamily/ixp2xxx.htm 


\title{
A Variation-tolerant Subthreshold Design Approach
}

Nikhil Jayakumar, Sunil P. Khatri

Department of Electrical Engineering, Texas A\&M University, College Station, TX

\begin{abstract}
Due to their extreme low power consumption, sub-threshold design approaches are appealing for a widening class of applications which demand low power consumption and can tolerate larger circuit delays. However, sub-threshold circuits are extremely sensitive to variations in supply, temperature and processing factors. In this paper, we present a sub-threshold design methodology which dynamically self-adjusts for inter and intra-die process, supply voltage and temperature (PVT) variations. This adjustment is achieved by performing bulk voltage adjustments in a closed-loop fashion, using a charge pump and a phase-detector.

Keywords: Sub-threshold, Body-biasing, Self-adjusting, Variation-tolerant
\end{abstract}

\section{REFERENCES}

[1] H. Soeleman, K. Roy, and B. Paul, "Robust subthreshold logic for ultra-low power operation," IEEE Transactions on Very Large Scale Integration (VLSI) Systems, vol. 9, pp. 90-99, Feb 2001.

[2] H. Soeleman and K. Roy, "Digital CMOS logic operation in the sub-threshold region," in Tenth Great Lakes Symposium on VLSI, pp. 107-112, Mar 2000.

[3] H. Soeleman and K. Roy, "Ultra-low power digital subthreshold logic circuits," in International Symposium on Low Power Electronic Design, pp. 94-96, 1999.

[4] B. Paul, H. Soeleman, and K. Roy, "An 8x8 sub-threshold digital CMOS carry save array multiplier," in European Solid State Circuits Conference, Sept 2001.

[5] J. Tschanz, J. Kao, S. Narendra, R. Nair, D. Antoniadis, A. Chandrakasan, and V. De, "Adaptive body bias for reducing impacts of die-to-die and within-die parameter variations on microprocessor frequency and leakage," in IEEE Journal of Solid-State Circuits, vol. 37, pp. 1396-1402, Nov 2002.

[6] L. Nagel, "Spice: A computer program to simulate computer circuits," in University of California, Berkeley UCB/ERL Memo M520, May 1995.

[7] Y. Cao, T. Sato, D. Sylvester, M. Orshansky, and C. Hu, "New paradigm of predictive MOSFET and interconnect modeling for early circuit design," in Proc. of IEEE Custom Integrated Circuit Conference, pp. 201204, Jun 2000. http://www-device.eecs.berkeley.edu/ ptm.

[8] P. Zarkesh-Ha, T. Mule, and J. D. Meindl, "Characterization and modelling of clock skew with process variation," in IEEE 1999 Custom Integrated Circuits Conference, 1999.

[9] S. Khatri, A. Mehrotra, R. Brayton, A. Sangiovanni-Vincentelli, and R. Otten, "A novel VLSI layout fabric for deep sub-micron applications," in Proceedings of the Design Automation Conference, (New Orleans), June 1999.

[10] S. Khatri, R. Brayton, and A. Sangiovanni-Vincentelli, "Cross-talk immune VLSI design using a network of PLAs embedded in a regular layout fabric," in IEEE/ACM International Conference on Computer Aided Design, pp. 412-418, Nov 2000.

[11] N. Jayakumar and S. Khatri, "A METAL and VIA maskset programmable VLSI design methodology using PLAs," in IEEE/ACM International Conference on Computer Aided Design, pp. 590-594, Nov 2004. 


\title{
Leakage Efficient Chip-Level Dual-Vdd Assignment with Time Slack Allocation for FPGA Power Reduction
}

\author{
Yan Lin and Lei He \\ Electrical Engineering Department, University of California, Los Angeles, CA
}

\begin{abstract}
To reduce power, Vdd programmability has been proposed recently to select Vdd-level for interconnects and to powergate unused interconnects. However, Vdd-level converters used in the Vdd-programmable method consume a large amount of leakage. In this paper, we develop chiplevel dual-Vdd assignment algorithms to guarantee that no low-Vdd interconnect switch drives high-Vdd interconnect switches. This removes the need of Vdd-level converters and reduces interconnect leakage and interconnect device area by $91.78 \%$ and $25.48 \%$, respectively. The assignment algorithms include power sensitivity based heuristics with implicit time slack allocation and a linear programming (LP) based method with explicit time slack allocation. Both first allocate time slack to interconnects with higher transition density and assign low-Vdd to them for more power reduction. Compared to the aforementioned Vdd-programmable method using Vdd-level converters, the LP based algorithm reduces interconnect power by 65.13\% without performance loss for the MCNC benchmark circuits. Compared to the LP based algorithm, the sensitivity based heuristics can obtain slightly smaller power reduction but run $4 X$ faster.
\end{abstract}

Keywords: FPGA, low power, time slack, programmable-Vdd

\section{REFERENCES}

[1] K. Poon, A. Yan, and S. Wilton, "A flexible power model for FPGAs," in Proc. of 12th International conference on Field-Programmable Logic and Applications, Sep 2002.

[2] F. Li, D. Chen, L. He, and J. Cong, "Architecture evaluation for power-efficient FPGAs," in Proc. ACM Intl. Symp. Field-Programmable Gate Arrays, Feb 2003.

[3] J. H. Anderson, F. N. Najm, and T. Tuan, "Active leakage power optimization for FPGAs," in Proc. ACM Intl. Symp. Field-Programmable Gate Arrays, Februray 2004.

[4] J. Lamoureux and S. J. Wilton, "On the interaction between power-aware FPGA CAD algorithms," in Proc. Intl. Conf. Computer-Aided Design, pp. 701-708, November 2003.

[5] A. Gayasen, Y. Tsai, N. Vijaykrishnan, M. Kandemir, M. J. Irwin, and T. Tuan, "Reducing leakage energy in FPGAs using region-constrained placement," in Proc. ACM Intl. Symp. Field-Programmable Gate Arrays, February 2004.

[6] F. Li, Y. Lin, L. He, and J. Cong, "Low-power FPGA using pre-defined dual-vdd/dual-vt fabrics," in Proc. ACM Intl. Symp. Field-Programmable Gate Arrays, Februray 2004.

[7] F. Li, Y. Lin, and L. He, "FPGA power reduction using configurable dual-vdd," in Proc. Design Automation Conf., June 2004.

[8] Fei Li and Yan Lin and Lei He, "Vdd programmability to reduce FPGA interconnect power," in Proc. Intl. Conf. Computer-Aided Design, November 2004.

[9] Jason H. Anderson and Farid N. Najm, "Low-power programmable routing circuitry for FPGAs," in Proc. Intl. Conf. Computer-Aided Design, November 2004.

[10] Y. Lin, F. Li, and L. He, "Power modeling and architecture evaluation for FPGA with novel circuits for vdd programmability," in Proc. ACM Intl. Symp. Field-Programmable Gate Arrays, Februray 2005.

[11] Y. Lin and L. He, "Leakage efficient chip-level dual-vdd assignment with time slack allocation for FPGA power reduction,” Tech. Rep. 05-257, UCLA Engr., available at http://eda.ee.ucla.edu

[12] V. Betz, J. Rose, and A. Marquardt, Architecture and CAD for Deep-Submicron FPGAs. Kluwer Academic Publishers, Feb 1999.

[13] M Berkelaar, lp-solver 3.2: a public domain (MI)LP solver. ftp://ftp.ics.ele.tue.nl/pub/lp solve/. 


\begin{abstract}
In this paper we present a system level technique for mapping large, multiple-IP-block designs to channel-width constrained FPGAs. Most FPGA clustering tools [2, 3, 11] aim to reduce the amount of inter-cluster connections, hence reducing channel width needs. However, if this exceeds the FPGA's channel width (a hard constraint), then the circuit still cannot be routed. Previous work [11, 12] depopulates logic clusters (CLBs) to reduce channel width. By depopulating non-uniformly, i.e. depopulate more in hard-to-route regions, we show a graceful trade-off between channel width and CLB count. This makes it possible to target specific channel-width constraints during clustering with minimal CLB inflation. Results show channel width decreases of up to $20 \%$ with a $5 \%$ increase in area. Further decreases of nearly $50 \%$ are possible at 3.3 times the original area. Despite the area increase, this technique creates routable solutions from otherwise-unroutable circuits.

Keywords: Field-Programmable Gate Arrays (FPGA), Clustering, Packing, Channel Width Constraints
\end{abstract}

\title{
REFERENCES
}

[1] E. Ahmed and J. Rose. The effect of LUT and cluster size on deep-submicron FPGA performance and density. In Int'l Symp. on FPGAs, pages 3-12, 2000.

[2] V. Betz, J. Rose, and A. Marquardt. Architecture and CAD for Deep-Submicron FPGAs. Kluwer Academic Publishers, Boston, 1999.

[3] E. Bozorgzadeh, S. Ogrenci-Memik, et al. Routability-driven packing: Metrics and algorithms for cluster-based FPGAs. Journal of Circuits, Systems, and Computers, 13(1):77-100, February 2004.

[4] Collaborative Benchmarking Laboratory. LGSynth93 suite. North Carolina State University.

[5] J. Cong and Y. Ding. FlowMap: An optimal technology mapping algorithm for delay optimization in lookuptable based FPGA designs. IEEE Trans. on Computer-Aided Design, pages 1-12, January 1994.

[6] A. DeHon. Balancing interconnect and computation in a reconfigurable computing array. In Int'l Symp. on FPGAs, pages 69-78, 1999.

[7] M. Hutton, J. Rose, and D. Corneil. Automatic generation of synthetic sequential benchmark circuits. IEEE Trans. on Computer-Aided Design, 21(8), August 2002.

[8] P. Kundarewich and J. Rose. Synthetic circuit generation using clustering and iteration. IEEE Trans. on Computer-Aided Design, 23(6), June 2004.

[9] P. Leventis, M. Chan, et al. Cyclone: A low-cost, high-performance FPGA. In IEEE Custom Integrated Circuits Conference, San Jose, CA, September 2003.

[10] D. Lewis, E. Ahmed, et al. The Stratix II logic and routing architecture. In Int'l Symp. on FPGAs, Monterey, CA, February 2005.

[11] A. Singh and M. Marek-Sadowska. Efficient circuit clustering for area and power reduction in FPGAs. In Int'l Symp. on FPGAs, pages 59-66, 2002.

[12] R. Tessier and H. Giza. Balancing logic utilization and area efficiency in FPGAs. In Int'l Workshop on Field Programmable Logic and Applications, 2000.

[13] P. Verplaetse, D. Stroobandt, and J. van Campenhout. Synthetic benchmark circuits for timing-driven physical design applications. In Int'l Conference on VLSI, pages 31-37, Las Vegas, NV, June 2002. 


\title{
Dynamic Reconfiguration with Binary Translation: Breaking the ILP Barrier with Software Compatibility
}

\author{
Antonio Carlos S. Beck, Luigi Carro \\ Universidade Federal do Rio Grande do Sul \\ Instituto de Informática, Campus do Vale, Porto Alegre, Brasil
}

\begin{abstract}
In this paper we present the impact of dynamically translating any sequence of instructions into combinational logic. The proposed approach combines a reconfigurable architecture with a binary translation mechanism, being totally transparent for the software designer. Besides ensuring software compatibility, the technique allows porting the same code for different machines tracking technological evolutions. The target processor is a Java machine able to execute Java bytecodes. Experimental results show that even code without any available parallelism can benefit from the proposed approach. Algorithms used in the embedded systems domain were accelerated 4.6 times in the mean, while spending 10.89 times less energy in the average. We present results regarding the impact of area and power, and compare the proposed approach with other Java machines, including a VLIW one.
\end{abstract}

Keywords: Java, Reconfigurable Processors, Binary Translation, Power Consumption

\section{REFERENCES}

[1] Schlett, M., "Trends in Embedded-Microprocessor Design". In Computer, vol. 31, n. 8, 1998, 44-49

[2] Nokia N-GAGE Home Page, available at http://www.n-gage.com

[3] Stitt, G., Lysecky, R., Vahid, F., "Dynamic Hardware/Software Partitioning: A First Approach". In Design Automation Conference (DAC), 2003

[4] Lysecky, R., Vahid, F., "A Configurable Logic Architecture for Dynamic Hardware/Software Partitioning”. In Design Automation And Test in Europe Conference (DATE), 2004

[5] Gschwind, M., Altman, E., Sathaye, P., Ledak, Appenzeller, D., "Dynamic and Transparent Binary Translation". In IEEE Computer, vol. 3 n. 33, 2000, 54-59

[6] Bingxiong $\mathrm{Xu}$, Albonesi, D., "Runtime Reconfiguration Techniques for Efficient General-Purpose Computation". In Design \& Test of Computers, vol. 17, n. 1, Jan.-Mar. 2000, $42-52$

[7] Tiwari, V., Malik, S., Wolfe, A., "Power Analysis of Embedded Software: A First Step Towards Software Power Minimization". In IEEE Transactions on VLSI Systems, vol. 2, n. 4, Dec. 1994, 437-445

[8] Henkel, J., Ernst, R., "A Hardware/Software Partitioner using a Dynamically Determined Granularity". In Design Automation Conference, 1997

[9] Venkataramani, G., Najjar, W., Kurdahi, F., Bagherzadeh, N., Bohm W., "A Compiler Framework for Mapping Applications to a Coarsegrained Reconfigurable Computer Architecture. Conf. on Compiler". In Architecture and Synthesis for Embedded Systems (CASES), 2001

[10] Henkel, J., "A low power hardware/software partitioning approach for core-based embedded systems". In Design Automation Conference, 1999

[11] Stitt, G., Vahid F., "The Energy Advantages of Microprocessor Platforms with On-Chip Configurable Logic". In IEEE Design and Test of Computers, 2002

[12] Hauck, S., Fry, T., Hosler, M., Kao, J., “The Chimaera reconfigurable functional unit”. In Proc. IEEE Symp. FPGAs for Custom Computing Machines, 1997, 87-96.

[13] Kastrup, B., Bink, A., Hoogerbrugge, J., "ConCISe: a compiler-driven CPLD-based instruction set accelerator”. In Proc. 7th Annu. IEEE Symp Field-Programmable Custom Computing Machines, Napa Valley, 92-100.

[14] Klaiber, A., "The Technology Behind Crusoe Processors". In Transmeta Corporation White Paper, 2000.

[15] Stitt, G., Vahid, F., "Hardware/Software Partitioning of Software Binaries". In IEEE/ACM International Conference on Computer Aided Design, 2002

[16] Takahashi, D., "Java Chips Make a Comeback". In Red Herring, 2001

[17] Lawton, G., "Moving Java into Mobile Phones". In Computer, vol. 35, n. 6, 2002, 17-20

[18] Beck, A.C.S., Carro, L. "Low Power Java Processor for Embedded Applications". In: IFIP 12th International Conference on Very Large Scale Integration, Germany, December, 2003 
[19] Ito, S.A., Carro, L., Jacobi, R.P. "Making Java Work for Microcontroller Applications". In IEEE Design \& Test of Computers, vol. 18, n. 5, 2001, 100-110

[20] Beck, A.C.S., Carro, L. "A VLIW Low Power Java Processor for Embedded Applications". In 17th Brazilian Symp. Integrated Circuit Design (SBCCI 2004), Sep. 2004

[21] Callaway, T. K., Swartzlander Jr, E. E., "Power Delay Characteristics of Multipliers". In IEEE 13th Symposium on Computer Arithmetic (ARITH '97), Mar. 1997

[22] Beck, A.C.S., Mattos, J.C.B., Wagner, F.R., Carro, L. "CACO-PS: A General Purpose Cycle-Accurate Configurable Power-Simulator”. In 16th Brazilian Symp. Integrated Circuit Design, Sep. 2003

[23] Chen, R., Irwin, M. J., Bajwa, R., "Architecture-Level Power Estimation and Design Experiments". In $A C M$ Transactions on Design Automation of Electronic Systems, v. 6, n. 1, Jan. 2001, 50-66

[24] Leonardo Spectrum, available at homepage: http://www.mentor.com 


\title{
Beyond Safety: Customized SAT-based Model Checking
}

Malay K Ganai, Aarti Gupta and Pranav Ashar

NEC Laboratories America, Princeton, NJ USA

\begin{abstract}
Model checking of safety properties has taken a significant lead over non-safety properties in recent years. To bridge the gap, we propose dedicated SAT-based model checking algorithms for properties beyond safety. Previous bounded model checking (BMC) approaches have relied on either converting such properties to safety checking, or finding proofs by deriving termination criteria using loop-free path analysis. Instead, our approach uses a customized SAT-based formulation for bounded model checking of non-safety properties, and determines the completeness bounds for liveness using unbounded SAT-based analysis. Our main contributions are: 1) Customized property translations for LTL formulas for BMC, with novel features that utilize partitioning, learning, and incremental formulation. Customized translations not only improve the BMC performance significantly in comparison to standard monolithic LTL translations, but also allow efficient derivation and use of completeness bounds. Though we discuss the translation schemas for liveness, they can be easily extended to handle other LTL properties as well. 2) Customized formulations for determining completeness bounds for liveness using SAT-based unbounded model checking (UMC) rather than using loop-free path analysis. These formulations comprise greatest fixed-point and least fixed-point computations to efficiently handle nested properties using SAT-based quantification approaches. We show the effectiveness of our overall approach for checking liveness on public benchmarks and several industry designs.
\end{abstract}

Keywords: Formal verification, bounded model checking, unbounded model checking, LTL, liveness, SAT, circuit cofactoring

\section{REFERENCES}

[1] B. Alpern and F. B. Schneider, "Defining liveness," Information Processing Letters, 1985.

[2] A. Biere, A. Cimatti, E. M. Clarke, and Y. Zhu, "Symbolic Model Checking without BDDs," in Proceedings of TACAS, 1999.

[3] A. Cimatti, M. Pistore, M. Roveri, and R. Sebastiani, "Improving the Encoding of \{LTL\} Model Checking into SAT," Proceedings of VMCAI, 2002.

[4] M. Sheeran, S. Singh, and G. Stalmarck, "Checking Safety Properties using Induction and a SAT Solver," in Proceedings of FMCAD, 2000.

[5] A. Gupta, M. Ganai, C. Wang, Z. Yang, and P. Ashar, "Abstraction and Bdds Complement SAT-Based BMC in DiVer," in Proceedings of CAV, 2003.

[6] A. Gupta, Z. Yang, P. Ashar, and A. Gupta, "SAT-based Image Computation with Application in Reachability Analysis," in Proceedings of FMCAD, 2000.

[7] K. McMillan, "Applying SAT methods in Unbounded Symbolic Model Checking," in Proceedings of CAV, 2002.

[8] K. McMillan, "Interpolation and SAT-based Model Checking," in Proceedings of CAV, 2003.

[9] M. Ganai, A. Gupta, and P. Ashar, "Efficient SAT-based Symbolic Unbounded Model Checking Using Circuit Cofactoring," in Proceedings of ICCAD, 2004.

[10] K. McMillan and N. Amla, "Automatic Abstraction without Counterexamples," in Proceedings of TACAS, 2003.

[11] A. Gupta, M. Ganai, P. Ashar, and Z. Yang, "Iterative Abstraction using SAT-based BMC with Proof Analysis," in Proceedings of ICCAD, 2003.

[12] E. M. Clarke, O. Grumberg, and D. Peled, Model Checking: MIT Press, 1999.

[13] K. L. McMillan, Symbolic Model Checking: An Approach to the State Explosion Problem: Kluwer Academic Publishers, 1993.

[14] "Liveness Manifesto. Beyond Safety International Workshop. 2004." 
[15] P. Wolper, M. Y. Vardi, and A. P. Sistla, "Reasoning about infinite computation paths.," Proceedings of Symposium on FCS, 1983.

[16] R. Gerth, D. Peled, M. Y. Vardi, and P. Wolper, "Simple on-the-fly automatic verification of linear temporal logic," Protocol Specification, Testing and Verification, 1995.

[17] M. Daniele, F. Giunchiglia, and M. Y. Vardi, "Improved automata generation for linear time temporal logic," Proceedings of CAV, 1999.

[18] F. Somenzi and R. Bloem, "Efficient Buchi Automata from LTL formulae," Proceedings of CAV, 2000.

[19] D. Kroening and O. Shtrichman, "Efficient computation of recurrence diameter," Proceedings of VMCAI, 2003.

[20] A. Biere, C. Artho, and V. Schuppan, "Liveness Checking as Safety Checking," Proceedings of FMICS, 2002.

[21] M. Awedh and F. Somenzi, "Proving more properties with Bounded Model Checking," Proceedings of CAV, 2004.

[22] O. Shtrichman, "Pruning Techniques for the SAT-based bounded model checking," in Proceedings of TACAS, 2001.

[23] J. Whittemore, J. Kim, and K. Sakallah, "SATIRE: A New Incremental Satisfiability Engine," Proceedings of $D A C, 2001$.

[24] M. Ganai and A. Aziz, "Improved SAT-based Bounded Reachability Analysis," in Proceedings of VLSI Design Conference, 2002.

[25] M. Ganai, L. Zhang, P. Ashar, and A. Gupta, "Combining Strengths of Circuit-based and CNF-based Algorithms for a High Performance SAT Solver," in Proceedings of DAC, 2002.

[26] "The VIS Home Page. http://wwwcad.eecs.berkeley.edu/Respep/Research/vis/."

[27] M. Moskewicz, C. Madigan, Y. Zhao, L. Zhang, and S. Malik, "Chaff: Engineering an Efficient SAT Solver," in Proceedings of DAC, 2001.

[28] M. Ganai, A. Gupta, and P. Ashar, "DiVer: SAT-based Model Checking Platform for Verifying Large Scale Systems", in Proceedings of TACAS, 2005. 


\title{
Efficient SAT Solving: Beyond Supercubes
}

\author{
Domagoj Babić, Jesse Bingham, Alan J. Hu \\ Department of Computer Science, University of British Columbia
}

\begin{abstract}
SAT (Boolean satisfiability) has become the primary Boolean reasoning engine for many EDA applications, so the efficiency of SAT solving is of great practical importance. Recently, Goldberg et al. introduced supercubing, a different approach to search-space pruning, based on a theory that unifies many existing methods. Their implementation reduced the number of decisions, but no speedup was obtained. In this paper, we generalize beyond supercubes, creating a theory we call B-cubing, and show how to implement B-cubing in a practical solver. On extensive benchmark runs, using both real problems and synthetic benchmarks, the new technique is competitive on average with the newest version of ZChaff, is much faster in some cases, and is more robust. Categories and Subject Descriptors
\end{abstract}

Keywords: SAT, formal verification, learning, search space pruning

\section{REFERENCES}

[1] D. Babić and A. J. Hu. Integration of Supercubing and Learning in a SAT Solver. In Asia South Pacific Design Automation Conference, pages 438-444. ACM/IEEE, 2005.

[2] F. Bacchus and J. Winter. Effective Preprocessing with Hyper-Resolution and Equality Reduction. In SAT, pages 341-355, 2003.

[3] A. Bhalla, I. Lynce, J. de Sousa, and J. Marques-Silva. Heuristic backtracking algorithms for SAT. In $4^{\text {th }}$ International Workshop on Microprocessor Test and Verification, pages 69- 74, 2003.

[4] A. Biere. The Evolution from LIMMAT to NANOSAT. Technical Report 444, Dept. of Computer Science, ETH Zürich, 2004.

[5] A. Biere, A. Cimatti, E. M. Clarke, M. Fujita, and Y. Zhu. Symbolic model checking using SAT procedures instead of BDDs. In 36th ACM/IEEE Design Automation Conference, pages 317-320. ACM Press, 1999.

[6] R. E. Bryant. Graph-based algorithms for boolean function manipulation. IEEE Trans. Comput., 35(8):677-691, 1986.

[7] M. Davis and H. Putnam. A Computing Procedure for Quantification Theory. J. ACM, 7(3):201-215, 1960.

[8] D.-Z. Du and K. Ko. Theory of Computational Complexity. John Wiley and Sons, 2000.

[9] E. Goldberg, M. R. Prasad, and R. K. Brayton. Using Problem Symmetry in Search Based Satisfiability Algorithms. In Proceedings of the conference on Design, Automation, and Test in Europe, pages 134-142, 2002.

[10] J. N. Hooker and V. Vinay. Branching rules for satisfiability. Journal of Automated Reasoning, 15(3):359-383, 1995.

[11] S. i. Minato. Zero-suppressed bdds for set manipulation in combinatorial problems. In 30th Design Automation Conference, pages 272-277. ACM Press, 1993.

[12] J. P. Marques-Silva and K. A. Sakallah. GRASP: A Search Algorithm for Propositional Satisfiability. IEEE Trans. Comput., 48(5):506-521, 1999.

[13] K. L. McMillan. Interpolation and SAT-based model checking. In CAV 03: Computer-Aided Verification, LNCS 2725, pages 1-13. Springer, 2003.

[14] M. W. Moskewicz, C. F. Madigan, Y. Zhao, L. Zhang, and S. Malik. Chaff: engineering an efficient SAT solver. In Proceedings of the Design Automation Conference, pages 530-535. ACM Press, 2001.

[15] A. Nadel. Backtrack Search Algorithms for Propositional Logic Satisfiability: Review and Innovations. Master's thesis, Tel-Aviv University, 2002.

[16] G. Nam, K. Sakallah, and R. Rutenbar. A Boolean satisfiability-based incremental rerouting approach with application to FPGAs. In Proceedings of the conference on Design, Automation and Test in Europe, pages 560-565. IEEE Press, 2001.

[17] M. R. Prasad. Propositional Satisfiability Algorithms in EDA Applications. PhD thesis, University of California at Berkeley, 2001.

[18] L. Ryan. Efficient algortihtms for clause-learning SAT solvers. Master's thesis, Simon Fraser University, 2004.

[19] P. Stephan, R. Brayton, and A. Sangiovanni-Vincentelli. Combinational test generation using satisfiability. IEEE Transactions on Computer-Aided Design of Integrated Circuits and Systems, 15(9):1167-1176, Sept 1996. 
[20] J. P. Warners and H. van Maaren. A two phase algorithm for solving a class of hard satisfiability problems. Operations Research letters, 23:81-88, 1998.

[21] J. P. Warners and H. van Maaren. Recognition of tractable satisfiability problems through balanced polynomial representations. In 5th Twente Workshop on Graphs and Combinatorial Optimization, pages 229-244. Elsevier Science Publishers B. V., 2000.

[22] H. Zhang and M. E. Stickel. An efficient Algorithm for Unit Propagation. In Proceedings of the Fourth International Symposium on Artificial Intelligence and Mathematics (AI-MATH'96), Fort Lauderdale (Florida USA), 1996.

[23] L. Zhang, C. F. Madigan, M. H. Moskewicz, and S. Malik. Efficient conflict driven learning in a boolean satisfiability solver. In Proceedings of the International Conference on Computer-Aided Design, pages 279-285. IEEE Press, 2001.

[24] L. Zhang and S. Malik. The quest for efficient Boolean satisfiability solvers. In Proceedings of the 18th International Conference on Automated Deduction, pages 295-313. Springer-Verlag, 2002. 


\title{
Prime Clauses for Fast Enumeration of Satisfying Assignments to Boolean Circuits
}

HoonSang Jin, Fabio Somenzi

University of Colorado at Boulder

\begin{abstract}
Finding all satisfying assignments of a propositional formula has many applications in the design of hardware and software. An approach to this problem augments a clause-recording propositional satisfiability solver with the ability to add blocking clauses, which prevent the solver from visiting the same solution more than once. One generates a blocking clause from a satisfying assignment by taking its complement. In this paper, we present an improved algorithm for finding all satisfying assignments for a generic Boolean circuit. An optimization based on lifting-which generates minimal satisfying assignments-yields prime blocking clauses. Thanks to the primality of the blocking clauses, the derived conflict clauses usually prune both satisfiable and unsatisfiable points at once. The efficiency of our new algorithm is demonstrated by our preliminary results on SAT-based unbounded model checking.
\end{abstract}

Keywords: SAT, CNF, AllSAT, minimal satisfying assignment

\section{References}

[1] R. K. Brayton et al. VIS: A system for verification and synthesis. In $C A V^{\prime} 96$, pages 428-432. Springer-Verlag, Berlin, 1996. LNCS 1102.

[2] M. Davis, G. Logemann, and D. Loveland. A machine program for theorem proving. Communications of the ACM, 5:394-397, 1962.

[3] M. Davis and H. Putnam. A computing procedure for quantification theory. Journal of the ACM, 7(3):201-215, July 1960.

[4] N. Eén and N. Sörensson. Temporal induction by incremental SAT solving. First Intl.Workshop on Bounded Model Checking. ENTCS, 89(4), 2003.

[5] M. K. Ganai, A. Gupta, and P. Ashar. Efficient SAT-based unbounded symbolic model checking using circuit cofactoring. In ICCAD, pages 408-415, Nov. 2004.

[6] H. Jin, H. Han, and F. Somenzi. Efficient conflict analysis for finding all satisfying assignments of a Boolean circuit. In TACAS'05, pages 287-300, Apr. 2005. LNCS 3440.

[7] H. Jin and F. Somenzi. An incremental algorithm to check satisfiability for bounded model checking. Second Intl.Workshop on Bounded Model Checking. Boston, MA, July 2004.

[8] K. L. McMillan. Applying SAT methods in unbounded symbolic model checking. In CAV'02, pages 250-264. Springer-Verlag, Berlin, July 2002. LNCS 2404.

[9] K. Ravi and F. Somenzi. Minimal assignments for bounded model checking. In TACAS'04, pages 31-45, Barcelona, Spain, Mar.-Apr. 2004. LNCS 2988.

[10] URL: http://vlsi.colorado.edu/ vis. 


\title{
Dynamic Abstraction Using SAT-based BMC
}

\author{
Liang Zhang $\dagger$, Mukul R Prasad $\neq$, Michael S Hsiao§, Thomas Sidle $\neq$ \\ $\dagger$ Cadence Design Systems, San Jose, CA \\ †Advanced CAD Technology, Fujitsu Laboratories of America, Sunnyvale, CA \\ $\S$ Department of Electrical \& Computer Engineering, Virginia Tech, Blacksburg, VA
}

\begin{abstract}
We propose a new dynamic method of abstraction, which can be applied during successive steps of the model checking algorithm to further reduce the model produced by traditional static abstraction methods. This is facilitated by information gathered from an analysis of the proof of unsatisfiability of SAT-based bounded model checking problems formulated on the original design. The dynamic abstraction effectively allows the model checker to work with smaller abstract models. Experiments on several industrial benchmarks demonstrate that dynamic abstraction can significantly improve both the performance and the capacity of typical abstraction refinement flows.
\end{abstract}

Keywords: Abstraction Refinement, Model Checking, SAT

\section{REFERENCES}

[1] P. Bjesse and J. Kukula. Using Counter Example Guided Abstraction Refinement to Find Complex Bugs. In Proc. of the Design Automation and Test in Europe Conf., pages 156-161, Feb. 2004.

[2] C. Wang et al. Improving Ariadne's Bundle by Following Multiple Threads in Abstraction Refinement. In Proc. of the Intl. Conf. on CAD, pages 408-415, Nov. 2003.

[3] E. Clarke, A. Biere, R. Raimi, and Y. Zhu. Bounded Model Checking Using Satisfiability Solving. Formal Methods in System Design, 19(1):7-34, July 2001.

[4] E. Clarke, A. Gupta, J. Kukula, and O. Strichman. SAT-based Abstraction Refinement Using ILP and Machine Learning Techniques. In Intl. Conf. on Computer Aided Verification, pages 265-279, July 2002.

[5] D. Wang et al. Formal Property Verification by Abstraction Refinement with Formal, Simulation and Hybrid Engines. In Proc. of the Design Automation Conf., pages 35-40, June 2001.

[6] A. Gupta, M. Ganai, Z. Yang, and P. Ashar. Iterative Abstraction Using SAT-based BMC with Proof Analysis. In Proc. of the Intl. Conf. on CAD, pages 416-423, Nov. 2003.

[7] R. P. Kurshan. Computer-Aided Verification of Coordinating Processes: The Automata-Theoretic Approach. Princeton University Press, 1995.

[8] B. Li and F. Somenzi. Efficient Computation of Small Abstraction Refinements. In Proc. of the Intl. Conf. on CAD, Nov. 2004.

[9] M. Glusman et al. Multiple-Counterexample Guided Iterative Abstraction Refinement: An Industrial Evaluation. In Tools and Algorithms for the Construction and Analysis of Systems, pages 176-191, April 2003.

[10] F. Y. C. Mang and P.-H. Ho. Abstraction Refinement by Controllability and Cooperativeness Analysis. In Proc. of the Design Automation Conf., pages 224-229, June 2004.

[11] K. L. McMillan and N. Amla. Automatic abstraction without counterexamples. In Tools and Algorithms for the Construction and Analysis of Systems, LNCS 2619, pages 2-17, April 2003.

[12] P. Chauhan et al. Automated Abstraction Refinement for Model Checking Large State Spaces using SAT based Conflict Analysis. In Formal Methods in Computer-Aided Design, pages 33-51, Nov. 2002.

[13] R. Brayton et al. VIS: A system for Verification and Synthesis. In Intl. Conf. on Computer Aided Verification, pages 428-432, July 1996.

[14] http://ee.princeton.edu/ chaff/zchaff.php, Dec. 2003.

[15] L. Zhang and S. Malik. Validating SAT Solvers using an Independent Resolution-based Checker: Practical Implementations and Other Applications. In Proc. of the Design Automation and Test in Europe, pages 880-885, March 2003. 


\title{
BEOL Variability and Impact on RC Extraction
}

\author{
Nagaraj NS, Tom Bonifield, Abha Singh, Clive Bittlestone, Usha Narasimha, \\ Viet Le, Anthony Hill \\ Texas Instruments Inc., Dallas TX
}

\begin{abstract}
Historically, Back End of Line (BEOL) or interconnect resistance and capacitance have been viewed as parasitic components. They have now become key parameters with significant impact on circuit performance and signal integrity. This paper examines the types of BEOL variations and their impact on $\mathrm{RC}$ extraction. The importance of modeling systematic effects in $\mathrm{RC}$ extraction is discussed. The need for minimizing the computational error in $\mathrm{RC}$ extraction before incorporating random process variations is emphasized.
\end{abstract}

Keywords: Process variation, Interconnect, Extraction

\section{Reference}

[1] Nagaraj NS et. al., "Benchmarks for Interconnect Parasitic Resistance and Capacitance” in proc. of ISQED 2003. 


\section{An Effective DFM Strategy Requires Accurate Process and IP Pre-Characterization}

Carlo Guardiani, Massimo Bertoletti, Nicola Dragone, Marco Malcotti, and Patrick McNamara PDF Solutions, San Jose, CA

Keywords: DFM, yield, test chips, yield models

\section{REFERENCES}

[1] C. Hess, L. Weiland, "Determination of Defect Size Distributions Based on Electrical Measurements at a Novel Harp Test Structure"; Proc. IEEE 1997 Int. Conference on Microelectronic Test Stuctures, Vol.10, March 1997

[2] C. Hess, D. Stashower, B. Stine, G. Verma, L. Weiland, "Fast Extraction of Killer Defect Density and Size Distribution Using a Single Layer Short Flow NEST Structure", IEEE 2000 Int. Conference on Microelectronic Test Structures, Vol.13, March 2000

[3] C. Hess, B. E. Stine, L. H. Weiland, T. Mitchell, M. Karnett, K. Gardner, "Passive Multiplexer Test Structure for Fast and Accurate Contact and Via Fail Rate Evaluation”, ICMTS 2002

[4] M. Quarantelli et al, "Characterization and Modeling of MOSFET Mismatch of a Deep Submicron Technology", IEEE 2003 Int. Conference on Microelectronic Test Structures 2003

[5] C. Guardiani, et al., "An Asymptotically Constant, Linearly Bounded Methodology for the Statistical Simulation of Analog Circuits Including Component Mismatch Effects" In Proc. IEEE/ACM 37th Design Automation Conference, Los Angeles (CA), Jun. 2000

[6] John Kibarian, et Al. "Design For manufacturability in Nanometer Era: System Implementation and Silicon Results", Proceedings of ISSCC, San Francisco, CA, Feb 2005 


\title{
Variation-Tolerant Circuits: Circuit Solutions and Techniques
}

Jim Tschanz, Keith Bowman, Vivek De

\author{
Circuit Research Lab, Intel Corporation, Hillsboro, OR
}

\begin{abstract}
Die-to-die and within-die variations impact the frequency and power of fabricated dies, affecting functionality, performance, and revenue. Variation-tolerant circuits and post-silicon tuning techniques are important for minimizing the impacts of these variations. This paper describes several circuit techniques that can be employed to ensure efficient circuit operation in the presence of ever-increasing variations.
\end{abstract}

Keywords: Parameter variation, high-performance design, body bias

\section{REFERENCES}

[1] S. Borkar et. al., "Design and reliability challenges in nanometer technologies," Proc. DAC 2004, p. 75.

[2] S. Borkar et. al., "Parameter variations and impact on circuits and microarchitecture," Proc. DAC 2003, pp. 338342.

[3] K. A. Bowman et. al., "Impact of die-to-die and within-die parameter fluctuations on the maximum clock frequency distribution for gigascale Integration," IEEE J. Solid-State Circuits, pp. 183-190, Feb. 2002.

[4] J. Tschanz et. al., "Adaptive body bias for reducing impacts of die-to-die and within-die parameter variations on microprocessor frequency and leakage”, IEEE J. Solid-State Circuits, pp. 1396-1402, Nov. 2002.

[5] J. Tschanz et. al., "Effectiveness of adaptive supply voltage and body bias for reducing impact of parameter variations in low power and high performance microprocessors," IEEE J. Solid-State Circuits, pp. 826-829, May 2003. 


\title{
On the Need for Statistical Timing Analysis
}

Farid N. Najm

ECE Dept., University of Toronto, Ontario, Canada

\begin{abstract}
Traditional corner analysis fails to guarantee a target yield for a given performance metric. However, recently proposed solutions, in the form of statistical timing analysis, which work by propagating delay distributions, do not conform to modern design methodology. Instead, new statistical techniques are needed to modify corner analysis in ways that overcome its weaknesses without violating usage models of timing tools in modern flows.
\end{abstract}

Keywords: Variability, Statistical timing analysis

\section{REFERENCES}

[1] M. Sengupta, et al. Application specific worst case corners using response surfaces and statistical models. In IEEE International Symposium on Quality Electronic Design, pages 351-356, San Jose, CA, March 22-24 2004.

[2] S. B. Samaan. The impact of device parameter variations on the frequency and performance of VLSI chips. In IEEE/ACM International Conference on Computer-Aided Design (ICCAD), pages 343-346, San Jose, CA, November 7-11 2004.

[3] F. N. Najm and N. Menezes. Statistical timing analysis based on a timing yield model. In ACM/IEEE 41st Design Automation Conference, pages 460-465, San Diego, CA, June 7-11 2004. 


\title{
CAD Tools for Variation Tolerance
}

\author{
David Blaauw and Kaviraj Chopra \\ University of Michigan, Ann Arbor, MI
}

\begin{abstract}
Process variability greatly affects power and timing of nanometer scale CMOS circuits, leading to parametric yield loss due to both timing and power constraint violations. This parametric yield loss will continue to worsen in future technologies as a result of increasing process variations [1] and the increased importance of leakage power. Hence, statistical techniques are required to maximize parametric yield under given power and frequency constraints. Recently, much progress has been reported in the area of statistical modeling of leakage power [6] and circuit timing [2-5]. These techniques are useful in analyzing the impact of process variations on performance and power in nanometer CMOS designs. In this extended abstract, we outline the need for statistical optimization methods.
\end{abstract}

Keywords: Yield, Variability, Design Flows

\section{REFERENCES}

[1] S. Nassif, "Design for variability in DSM technologies," IEEE ISQED, pp. 451-454, 2000.

[2] H. Chang and S. S. Sapatnekar, "Statistical timing analysis considering spatial correlations using a single PERT like traversal," ACM/IEEE ICCAD, pp. 621-625, 2003.

[3] C. Viswesweriah et al, "First-order incremental block-based statistical timing analysis," ACM/IEEE DAC, 2004.

[4] M. Orshansky, A. Bandyopadhyay, "Fast statistical timing analysis handling arbitrary delay correlations," ACM/IEEE DAC, pp. 337 - 342, DAC 2004.

[5] A. Agarwal, D. Blaauw, and V. Zolotov, "Statistical timing analysis for intra-die process variations with spatial correlations," IEEE ICCAD, pp. 900-907, 2003.

[6] R. Rao, A. Srivastava, D. Blaauw, and D. Sylvester, "Statistical analysis of subthreshold leakage current for VLSI circuits," IEEE Trans. VLSI Systems, pp.131-139, Feb. 2004.

[7] S. Borkar, et al, "Parameter variation and Impact on Circuits and Microarchitecture," ACM/IEEE DAC, pp.338$342,2003$. 


\title{
Are There Economic Benefits in DFM? \\ Matt Nowak*, Riko Radojcic** \\ *Qualcomm Inc, San Diego, CA \\ **Consultant, San Diego, CA
}

\begin{abstract}
A fabless company perspective is presented on the roles of the foundries, design entities and EDA providers in the DFM arena, and the requirements for measurement of the economic benefits of DFM

Keywords: DFM, Design for Manufacturability, Foundries, Fabless
\end{abstract}




\title{
A Generic Micro-Architectural Test Plan Approach for Microprocessor Verification
}

Allon Adir, Hezi Azatchi, Eyal Bin, Ofer Peled, Kirill Shoikhet

IBM Research Laboratory in Haifa, IBM Haifa Labs, Haifa University, Mt Carmel, Haifa

\begin{abstract}
Modern microprocessors share several common types of microarchitectural building blocks. The rising complexity of the microarchitecture increases the risk of bugs and the difficulty of achieving comprehensive verification. We propose a methodology to exploit the commonality in the different microprocessors to create a design-independent micro-architectural test plan. Our method allows the testing of the huge micro-architectural test space by using systematic partitioning, which offers a high level of comprehensiveness of the tested behaviors. We show how this method was used to find bugs during verification of an actual high-end microprocessor. Our results show the advantages of this approach over the more traditional test methods that use design specific test plans or that use tools with little micro-architectural knowledge for covering micro-architectural aspects of the design.
\end{abstract}

Keywords: Micro-architecture, Coverage, Generic Test Plan, Dynamic Verification, Test Generation

\section{REFERENCES}

[1] A. Aharon, D. Goodman, M. Levinger, Y. Lichtenstein, Y. Malka, C. Metzger, M. Molcho, and G. Shurek. Test program generation for functional verification of PowerPC processors in IBM. In Proceedings of the $32 \mathrm{nd}$ Design Automation Conference, pages 279-285, June 1995.

[2] D. V. Campenhout, T. Mudge, and J. P. Hayes. High-level test generation for design verification of pipelined microprocessors. In Proceedings of the 36th Design Automation Conference, pages 185-188, June 1999.

[3] L. Fournier, Y. Arbetman, and M. Levinger. Functional verification methodology for microprocessors using the Genesys test-program generator. In Proceedings of the 1999 Design, Automation and Test in Europe Conference (DATE), pages 434-441, March 1999.

[4] H. Iwashita, S. Kowatari, T. Nakata, and F. Hirose. Auto-matic test program generation for pipelined processors. In Proceedings of the International Conference on Computer Aided Design, pages 580-583, November 1994.

[5] K. Kohno and N. Matsumoto. A new verification methodology for complex pipeline behavior. In Proceedings of the $38^{\text {th }}$ Design Automation Conference, pages 816-821, 2001.

[6] S. Ur and Y. Yadin. Micro-architecture coverage directed generation of test programs. In Proceedings of the 36th Design Automation Conference, pages 175-180, June 1999.

[7] J.-T. Yen and Q. R. Yin. Multiprocessing design verification methodology for Motorola MPC74XX PowerPC microprocessor. In Proceedings of the 37th Design Automation Conference, pages 718-723, June 2000.

[8] A. Adir, E Bin, O. Peled, and A. Ziv. A Test Program Generator for Micro-Architecture Flow Verification. In Proceedings of the HLDVT 2003, pagse 23-28.

[9] Adir, A., Almog, E., Fournier, L., Marcus, E., Rimon, M., Vinov, M. and Ziv, A. Genesys-Pro: Innovations in Test Program Generation for Functional Processor Verification. IEEE Design \& Test of Computers, Mar-Apr. 2004, 84-93.

[10] A. Ziv . Cross-Product Functional Coverage Measurement with Temporal Properties-based Assertions. In Proceedings of the 2003 Design Automation and Test in Europe (DATE'03)

[11] R. Grinwald, E. Harel, M. Orgad, S. Ur and A. Ziv. User Defined Coverage - A Tool Supported Methodology for Design Verification. In Proceedings of the 35th Design Automation Conference 1998. pages 158-165

[12] M. Aharoni, S. Asaf, L Fournier, A. Koyfman and R. Nagel. FPgen A Deep-Knowledge Test Generator for Floating Point Verification, HLDVT 2003, pages 17-22

[13] C. May, E. Silha, R. Simpson and H. Warren. The PowerPC Architecture. In Morgan Kaufmann, 1994.

[14] Julia Dushina, Mike Benjamin, Daniel Geist. Semi-Formal Test Generation with Genevieve. DAC 2001. 
IODINE: A Tool to Automatically Infer Dynamic Invariants for Hardware Designs

\author{
Sudheendra Hangal*, Naveen Chandra*, Sridhar Narayanan**, Sandeep Chakravorty* \\ *Sun Microsystems, Bangalore, India \\ **P. A. Semi Inc, Santa Clara, CA
}

\begin{abstract}
We describe IODINE, a tool to automatically extract likely design properties using dynamic analysis. A practical bottleneck in the formal verification of hardware designs is the need to manually specify design-specific properties. IODINE presents a way to automatically extract properties such as state machine protocols, request-acknowledge pairs, and mutual exclusion between signals from design simulations. We show that dynamic invariant detection for hardware designs can infer relevant and accurate properties.

Keywords: Dynamic Invariants, Dynamic Analysis, Formal Specification

\section{REFERENCES}

[1] A. Biere, A. Cimatti, E. Clarke, and Y. Zhu. Symbolic model checking without BDDs. In Tools and Algorithms for the Construction and Analysis of Systems, LNCS, 1579:193-207, 1999.

[2] M. D. Ernst, J. Cockrell, W. G. Griswold, and D. Notkin. Dynamically discovering likely program invariants to support program evolution. IEEE Transactions on Software Engineering, 27(2):1-25, Feb 2001.

[3] S. Hangal and M. S. Lam. Tracking down software bugs using automatic anomaly detection. In Proceedings of the International Conference on Software Engineering, May 2002.

[4] S. Kapil, H. McGhan, and J. Lawrendra. A chip multithreaded processor for network-facing workloads. IEEE Micro, pages 20-30, March-April 2004.

[5] J. W. Nimmer and M. D. Ernst. Invariant inference for static checking: An empirical evaluation. In Proceedings of the ACM SIGSOFT 10th International Symposium on the Foundations of Software Engineering, pages 11-20, 2002.

[6] S. Pollock and J. Zhang. Early and automatic error detection with Verix formal functional verification.

[7] S. Bensalem, Y. Lakhnech, and H. Saidi. Powerful techniques for the automatic generation of invariants. In Proceedings of Computer Aided Verification. Springer Verlag, 1996.

[8] J. Yang and D. Evans. Automatically inferring temporal properties for program evolution. Fifteenth IEEE International Symposium on Software Reliability Engineering, November 2004.
\end{abstract}




\title{
VLIW - A Case Study of Parallelism Verification
}

\author{
Allon Adir ${ }^{1}$, Yaron Arbetman ${ }^{1}$, Bella Dubrov ${ }^{1}$, Yossi Lichtenstein ${ }^{1}$, Michal Rimon ${ }^{1}$, \\ Michael Vinov ${ }^{1}$, Massimo A Calligaro ${ }^{2}$, Andrew Cofler ${ }^{2}$, Gabriel Duffy ${ }^{2}$ \\ ${ }^{1}$ IBM Research Laboratory, Haifa, Israel \\ ${ }^{2}$ ST Microelectronics Design Center, Grenoble, France
}

\begin{abstract}
Parallelism in processor architecture and design imposes a verification challenge as the exponential growth in the number of execution combinations becomes unwieldy. In this paper we report on the verification of a Very Large Instruction Word processor. The verification team used a sophisticated test program generator that modeled the parallel aspects as sequential constraints, and augmented the tool with manually written test templates. The system created large numbers of legal stimuli, however the quality of the tests was proved insufficient by several post silicon bugs. We analyze this experience and suggest an alternative, parallel generation technique. We show through experiments the feasibility of the new technique and its superior quality along several dimensions. We claim that the results apply to other parallel architectures and verification environments.

Keywords: Functional verification, Processor verification, Test generation, VLIW, Parallelism

\section{REFERENCES}

[1] http://www.intel.com/pressroom/archive/releases/20040907corp.htm

[2] http://domino.research.ibm.com/comm/pr.nsf/pages/news.20041110_bluegene.html

[3] Adir, A. and Shurek, G. Generating Concurrent Test-Programs with Collisions for Multi-Processor Verification. In Proceedings of the IEEE International High Level Design Validation and Test Workshop (HLDVT'02), 2002, 7982.

[4] Sullivan, M., Wilson, P., Montemayor, C., Evers, R. and Yen, J. Multiprocessor Design Verification with Generated Realistic MP Programs. In Proceedings of IEEE 14'th Annual IPCCC, 1995, 389-395.

[5] Aharon, A., Goodman, D., Levinger, M., Lichtenstein, Y., Malka, Y., Metzger, C., Molcho, M. and Shurek, G. Test Program Generation for Functional Verification of PowerPC Processors in IBM. In Proceedings of 32nd Design Automation Conference (DAC '95), 1995, 279-285.

[6] Malandain, D., Palmen, P., Taylor, M., Aharoni, M., Arbetman, Y. An Effective and Flexible approach to Functional Verification of Processor Families. In Proceedings of the IEEE International High Level Design Validation and Test Workshop (HLDVT'02), 2002, 93-98

[7] http://www.st.com/st100

[8] Homewood, F., Faraboschi, P. ST200: A VLIW Architecture for Media-Oriented Applications. Microprocessor Forum, Oct 2000.

[9] Adir, A., Almog, E., Fournier, L., Marcus, E., Rimon, M., Vinov, M. and Ziv, A. Genesys-Pro: Innovations in Test Program Generation for Functional Processor Verification. IEEE Design \& Test of Computers, Mar-Apr. 2004, 84-93.

[10] Bin, E., Emek, R., Shurek, G. and Ziv, A. Using Constraint Satisfaction Formulations and Solution Techniques for Random Test Program Generation. IBM Systems Journal, Aug. 2002, 386-402.

[11] Palnitkar, S. Design Verification with e. Prentice Hall, 2003.

[12] Haque, F., Michelson, J. and Khan, K. The Art of Verification with Vera. Verification Central, 2001
\end{abstract}




\title{
StressTest: An Automatic Approach to Test Generation Via Activity Monitors
}

\author{
Ilya Wagner, Valeria Bertacco, Todd Austin
}

Advanced Computer Architecture Lab, The University of Michigan - Ann Arbor, MI

\begin{abstract}
The challenge of verifying a modern microprocessor design is an overwhelming one: Increasingly complex micro-architectures combined with heavy time-to-market pressure have forced microprocessor vendors to employ immense verification teams in the hope of finding the most critical bugs in a timely manner. Unfortunately, too often size doesn't seem to matter for verification teams, as design schedules continue to slip and microprocessors find their way to the marketplace with design errors. In this paper, we describe a simulation-based random test generation tool, called StressTest, that provides assistance in locating hard-to-find corner-case design bugs and performance problems. StressTest is based on a Markov-model-driven random instruction generator with activity monitors. The model is generated from the user-specified template programs and is used to generate the instructions sent to the design under test (DUT). In addition, the user specifies key activity points within the design that should be stressed and monitored throughout the simulation. The StressTest engine then uses closed-loop feedback techniques to transform the Markov model into one that effectively stresses the points of interest. In parallel, StressTest monitors the correctness of the DUT response to the supplied stimuli, and if the design behaves unexpectedly, a bug and a trace that leads to it are reported. Using two micro-architectures as example testbeds, we demonstrate that StressTest finds more bugs with less effort than open-loop random instruction test generation techniques.

Keywords: Architectural simulation, High-performance simulation, Directed-random simulation
\end{abstract}

\section{REFERENCES}

[1] Constrained-random test generation and functional coverage with Vera. Technical report, Synopsys, Inc, Feb. 2003.

[2] Specman elite - testbench automation, 2004. http://www.verisity.com/products/specman.html.

[3] A. Allan, D. Edenfeld, J. William H. Joyner, A. B. Kahng, M. Rodgers, and Y. Zorian. 2001 technology roadmap for semiconductors. IEEE Computer, pages 42-53, Jan. 2002.

[4] M. Behm, J. Ludden, Y. Lichtenstein, M. Rimon, and M. Vinov. Industrial experience with test generation languages for processor verification. DAC 2004, June 2004.

[5] B. Bentley. Validating the Intel Pentium 4 microprocessor. In DAC, Proceedings of Design Automation Conference, pages 224-228, 2001.

[6] E.A.Poe. Introduction to random test generation for processor verification. Technical report, Obsidian Software, 2002.

[7] J. M. L. et.al. Functional verification of the POWER4 microprocessor and POWER4 multiprocessor systems. IBM Journal of Research and Development, 46:53-76, Jan. 2002.

[8] S. Fine and A. Ziv. Coverage directed test generation for functional verification using bayesian networks. In DAC, Proceedings of Design Automation Conference, pages 286-281, June 2003.

[9] Y. Levhari. Verification of the PalmDSPCore using pseudo random techniques. Technical report, VeriSure Consulting, Ltd., 2002.

[10] I. Silas, I. Frumkin, E. Hazan, E. Mor, and G. Zobin. System-level validation of the Intel Pentium M processor. Intel Technology Journal, 07:38-43, May 2003.

[11] S. Tasiran, F. Fallah, D. G. Chinnery, S. J. Weber, and K. Keutzer. A functional validation technique: Biasedrandom simulation guided by observability-based coverage. ICCD, Proceedings of the International Conference on Computer Design, pages 82-88, 2001.

[12] S. Taylor, M. Quinn, D. Brown, N. Dohm, S. Hildebrandt, J. Huggins, and C. Ramey. Functional verification of a multiple-issue, out-of-order, superscalar Alpha processor: The DEC Alpha 21264 microprocessor. In $D A C$, Proceedings of Design Automation Conference, pages 638-644, 1998. 


\title{
Smart Diagnostics for Configurable Processor Verification
}

\author{
Sadik Ezer, Scott Johnson \\ Tensilica Inc., Santa Clara, CA
}

\begin{abstract}
This paper describes a novel technique called Embedded Test-bench Control (ETC), extensively used in the verification of Tensilica's latest configurable processor. Conventional simulationbased verification methodologies that employ assembly programs for testing cannot easily link the diagnostic program to the test-bench for interactive control, consequently resulting in weaker coverage. ETC links the diagnostic program execution and the test-bench functions, thereby increasing the flexibility and power of the diagnostics to create more complex corner cases in fewer simulation cycles and with smaller code size. This method also enables dynamic selfchecking and dynamic coverage analysis by either passing or failing the diagnostic based on the coverage goal, or terminating runaway random diagnostics much earlier. The presented simulation results show that ETC augments verification in two major areas: the creation of more maintainable, efficient, and smart diagnostics, and the reduction of the regression time. Some of the techniques presented in this paper can apply to non-processor verification methodologies as well.
\end{abstract}

Keywords: Functional Verification, Configurable Processors, Embedded Test-bench Control, Diagnostics, Coverage

\section{REFERENCES}

[1] Bergeron, J. Writing Testbenches: Functional Verification of HDL Models. Kluwer Academic Publishers, January 2000.

[2] Gluska, A. Coverage-Oriented Verification of Banias. ACM/IEEE Design Automation Conference, pages 280285, June 2003.

[3] Bentley, B. Validating the Intel Pentium 4 Microprocessor. ACM/IEEE Design Automation Conference, pages 244-248, June 2001.

[4] Jani, D., Ezer, G., and Kim, J. Long Words and Wide Ports. Hot Chips, August 2004.

[5] Rowen, C. Engineering the Complex SOC. Prentice-Hall PTR, 2004.

[6] Puig-Medina, M., Ezer, G., and Konas, P. Verification of Configurable Processors. ACM/IEEE Design Automation Conference, pages 426-431, June 2000.

[7] Behm, M., Ludden, J., Lichtenstein, Y., Rimon, M., and Vinov, M. Industrial Experience with Test Generation Languages for Processor Verification. ACM/IEEE Design Automation Conference, pages 36-40, June 2004.

[8] Gonzales, R. Xtensa: A Configurable and Extensible Processor. IEEE Micro, 20(2), March/April 2000.

[9] Ho, R., and Horowitz, M. Validation Coverage Analysis for Complex Digital Designs. IEEE/ACM International Conference of Computer Aided Design, 1996.

[10] Fine, S., Ur, S., and Ziv, A. Probabilistic Regression Suites for Functional Verification. ACM/IEEE Design Automation Conference, pages 49-54, June 2004.

[11] Iwashita, H. Automatic Test Program Generation for Pipelined Processors. IEEE/ACM International Conference on Computer-Aided Design, pages 580-588, 1994.

[12] Synopsys Inc., Mountain View, California, Vera ${ }^{\circledR}$ User Guide. March 2004

[13] Kohno, K., and Matsumoto, N. A New Verification Methodology for Complex Pipeline Behavior. ACM/IEEE Design Automation Conference, pages 49-54, June 2004.

[14] Hennenhoefer, E., Typaldos, M. The Evolution of Processor Test Generation Technology. Obsidian Software Inc. 
DAC05, pages 795- 800

\title{
Power-Aware Placement
}

\author{
Yongseok Cheon*, Pei-Hsin Ho*, Andrew B. Kahng †, Sherief Reda†, Qinke Wangt \\ *Advanced Technology Group, Synopsys, Inc. \\ †CSE Department, University of California at San Diego
}

\begin{abstract}
Lowering power is one of the greatest challenges facing the IC industry today. We present a power-aware placement method that simultaneously performs (1) activity-based register clustering that reduces clock power by placing registers in the same leaf cluster of the clock trees in a smaller area and (2) activity-based net weighting that reduces net switching power by assigning a combination of activity and timing weights to the nets with higher switching rates or more critical timing. The method applies to designs with multiple clocks and gated clocks. We implemented the method and obtained experimental results on 8 real-world designs after placement, routing, extraction and analysis. The power-aware placement method achieved on average $25.3 \%$ and $11.4 \%$ reduction in net switching power and total power respectively, with $2.0 \%$ timing, $1.2 \%$ cell area and $11.5 \%$ runtime impact. This method has been incorporated into a commercial physical design tool.

Keywords: Net Switching Power, Clock Tree, Dynamic Power
\end{abstract}

\section{REFERENCES}

[1] V. Adler and E. G. Friedman, "Repeater Insertion to Reduce Delay and Power in RC Tree Structures," IEEE Asilomar COnf. on Signals, Systems and Computers, 1997, pp. 749-752.

[2] L. Benini, P. Siefel, and G. D. Micheli, "Automatic Synthesis of Gated Clocks for Power Reduction in Sequential Circuits," IEEE Trans. on Computer-Aided Design of Integrated Circuits and Systems, 15(6) (1996), pp. 630-643.

[3] K.-Y. Chao and D.-F. Wong, "Floorplanning for Low Power Designs," Proc. IEEE Int. Symp. Circuits and Systems, 1(28) (1995), pp. 45-48.

[4] T. Chao, Y. Hsu, J. Ho, K. Boese, and A. Kahng, "Zero Skew Clock Routing with Minimum Wirelength," IEEE Trans. on Computer-Aided Design of Integrated Circuits and Systems, 39(11) (1992), pp. 799-814.

[5] J. Cong, C. K. Koh, and K. S. Leung, "Simultaneous Buffer and Wire Sizing for Performance and Power Optimization," ACM/IEEE Int. Symp. Low-Power Electronics and Design, 1996, pp. 271-276.

[6] M. Donno, E. Macci, and L. Mazzoni, "Power-Aware Clock Tree Planning," Proc. ACM/IEEE Int. Symp. Physical Design, 2004, pp. 138-147.

[7] A. Farrahi, C. Chen, A. Srivastava, G. Tellez, and M. Sarrafzadeh, “Activity-Driven Clock Design," IEEE Trans. on Computer-Aided Design of Integrated Circuits and Systems, 20(6) (2001), pp. 705-714.

[8] P. E. Gronowski, W. J. Bowhill, R. P. Preston, M. K. Gowan, and R. L. Allmon, "High-Performance Microproccesor Design," IEEE Journal of Solid-State Circuits, 33(5) (1998), pp. 676-686.

[9] M. Igarashi et al., "A Low-Power Design Method Using Multiple Supply Voltages," ACM/IEEE Int. Symp. LowPower Electronics and Design, 1999, pp. 145-150.

[10] M. Jackson, A. Srinivason, and E. Kuh, "Clock Routing for High-Perfomance ICs," Proc. ACM/IEEE Design Automation Conf., 1990, pp. 573-579.

[11] A. Kahng, J. Cong, and G. Robins, "High-Perfomance Clock Routing Based on Recursive Geometric Matching," Proc. ACM/IEEE Design Automation Conf., 1991, pp. 322-327.

[12] A. Krishnamoorthy, "Minimize IC Power without Sacrificing Performance," EEdesign, July 15, 2004. Available at http://www.eedesign.com/article/showArticle.jhtml?articleId=23901143.

[13] N. Magen, A. Kolodny, U. Weiser, and N. Shamir, "Interconnect-power Dissipation in a Microprocessor," Proc. Workshop on System Level Interconnect Prediction, 2004, pp. 7-13.

[14] B. Obermeier and F. M. Johannes, "Temperature-Aware Global Placement," Proc. Asia and South Pacific Design Automation Conf., 2004, pp. 143-148.

[15] J. Oh and M. Pedram, "Gated Clock Routing for Low-Power Microprocessor Design," IEEE Trans. on Computer-Aided Design of Integrated Circuits and Systems, 20(6) (2001), pp. 715-714.

[16] J. Pangjun and S. Sapatnekar, "Clock Distribution Using Multiple Voltages," Proc. ACM/IEEE Int. Symp. LowPower Electronics and Design, 1999, pp. 145-150. 
[17] S. M. Sait, M. R. Minhas and J. A. Khan, "Performance and Low Power Driven VLSI Standard Cell Placement Using Tabu Search," Proc. Congress on Evolutionary Computation, 2002, pp. 372-377.

[18] V. Tiwari, D. Singh, S. Rajgopal, G. Mehta, R. Patel, and F. Baez, "Reducing Power in High-Performance Microprocessors," Proc. ACM/IEEE Design Automation Conf., 1998, pp. 732-737.

[19] N. Togawa, K. Ukai, M. Yanagisawa and T. Ohtsuki, "A Simultaneous Placement and Global Routing Algorithm for FPGAs with Power Optimization," Proc. IEEE Asia-Pacific Conf. Circuits and Systems, 1998, pp. $125-128$.

[20] A. Vittal and M. Marek-Sadowska, "Low-Power Buffered Clock Tree Design," IEEE Trans. on ComputerAided Design of Integrated Circuits and Systems, 16(9) (1997), pp. 965-975.

[21] H. Vaishnav and M. Pedram, "PCUBE: A Performance Driven Placement Algorithm for Low Power Designs", Proc. Design Automation Conf. with EURO-VHDL, 1993, pp. 72-77. 


\title{
How Accurately Can We Model Timing In A Placement Engine?
}

\author{
Amit Chowdhary*, Karthik Rajagopal*, Satish Venkatesan*, Tung Cao*,Vladimir Tiourin*, \\ Yegna Parasuram $†$, Bill Halpin $\neq$ \\ *Intel Corporation, Santa Clara, CA \\ $\uparrow$ Sierra Design Automation, Santa Clara, CA \\ †Synplicity, Inc., Sunnyvale, CA
}

\begin{abstract}
This paper presents a novel placement algorithm for timing optimization based on a new and powerful concept, which we term differential timing analysis. Recognizing that accurate optimization requires timing information from a signoff static timing analyzer, we propose an incremental placement algorithm that uses timing information from a signoff static timing engine. We propose a set of differential timing analysis equations that accurately capture the effect of placement perturbations on changes in timing from the signoff timer. We have formulated an incremental placement optimization problem based on differential timing analysis as a single linear programming (LP) problem which is solved to generate the new timingoptimized placement.
\end{abstract}

Our experiments show that the worst negative slack (WNS) improves by an average of 30\% and the total negative slack (TNS) improves by $33 \%$ on average for a set of circuits from a $3.0 \mathrm{GHz}$ microprocessor that were already synthesized and placed by a leading industrial physical synthesis tool. We also show that multiple iterations of our engine give further TNS improvements - an average improvement of $51 \%$, which implies that our placer will significantly speed up timing convergence.

Keywords: Timing-driven placement, static timing analysis, linear programming, differential timing analysis

\section{REFERENCES}

[1] W. C. Elmore, "The transient response of Damped Linear network with particular regard to wideband amplifier", Journal of Applied Physics, pp.55-63, 1948.

[2] ILOG, ILOG CPLEX 8.0 User's Manual. ILOG, 2002.

[3] B. Halpin, C. Y. R. Chen, N. Sehgal, "Timing driven placement using physical net constraints", Proc. Design Automation Conf., pp. 780-783, 2001.

[4] K. Rajagopal, T. Shaked, Y. Parasuram, T. Cao, A. Chowdhary, B. Halpin, "Force directed timing driven placement with physical net constraints", Proc. Intl Symp. on Physical Design, pp. 147-152, 2003.

[5] A.H. Ajami, M. Pedram, "Post-layout timing driven cell placement using an accurate net length model", Proc. Design Automation Conf., pp. 595-600, 2001.

[6] A. B. Kahng, S. Mantik, I. L. Markov, "Min-max placement for large-scale timing optimization", Proc. Intl. Symp. of Physical Design, pp. 143-148, 2002.

[7] W.Choi, K.Bazargan, "Incremental Placement for Timing Optimization", Proc. Intl Conf. on CAD, 2003.

[8] C.-C. Chang, J.Cong, M. Xie, "Optimality and scalability study of existing placement algorithms", Proc. of the $A S P D A C$, Jan. 2003. 


\title{
Efficient and Accurate Gate Sizing with Piecewise Convex Delay Models
}

\author{
Hiran Tennakoon and Carl Sechen \\ Department of Electrical Engineering, University of Washington, Seattle WA
}

\begin{abstract}
We present an efficient and accurate gate sizing tool that employs a novel piecewise convex delay model, handling both rise and fall delays, for static CMOS gates. The delay model is used in a new version of a gate-sizing tool called Forge, which not only exhibits optimality, but also efficiently produces the area versus delay trade-off curve for a block in one step. Forge includes a realistic delay propagation scheme that combines arrival times and slew-rates. Forge is $6.4 \mathrm{X}$ faster than a commercial transistor sizing tool, while achieving better delay targets and uses $28 \%$ less transistor area for specific delay targets, on average.

Keywords: Delay modeling, gate sizing, Lagrangian relaxation, piecewise convex, optimization
\end{abstract}

\section{References}

[1] M. delM. Hershenson, S.P. Boyd, and T.H. Lee, "Optimal design of a CMOS op-amp via geometric programming," IEEE Trans. on Computer-Aided Design, vol. 20, no 1, pp. 1-21, Jan 2001.

[2] M. Vujkovic and C. Sechen "Optimized power-delay curve generation for standard cell ICs," Proc. Int'l. Conf. on Compter-Aided Des. (ICCAD), pp. 387-394, Nov 2002.

[3] M. Vujkovic, D Wadkins, B Shwartz, and C. Sechen, "Efficient timing closure without timing driven placement and routing," Proc. Des. Auto. Conf. (DAC), pp. 268-273, June 2004.

[4] M.R.C.M. Berkelaar, P.H.W. Buurman, and J. A. G. Jess, "Computing the entire active area/power consumption versus delay tradeoff curve for gate sizing with a piecewise linear simulator," IEEE Trans. on Computer-Aided Design, vol. 15, no. 11, pp. 1424-1434, Nov 1996.

[5] J. P. Fishburn and A. E. Dunlop, "TILOS: A posynomial programming approach to transistor sizing," IEEE Trans. on Computer-Aided Design, pp. 326-328, Nov 1985.

[6] W.C. Elmore, "The transient analysis of damped linear networks with particular regard to wideband amplifiers." Journal of Applied Physics, vol. 19, no. 1, pp. 55-63, 1948.

[7] K. Kasamsetty, M. Ketkar, and S. S. Sapatnekar, "A New Class of Convex Functions for Delay Modeling and their Application to the Transistor Sizing Problem," IEEE Trans. on Computer-Aided, vol. 19, no. 7, pp. 779-788, July 2000.

[8] Jim-Fuw Lee, D.L. Ostapko, J. Soreff, C.K. Wong, "On the signal bounding problem in timing analysis", Proc. Int'l Conf. on Compter-Aided Design, pp. 507-514, Nov 2001.

[9] S. S. Sapatnekar, V. B. Rao, P. M. Vaidya, and S. M. Kang, "An exact solution of the transistor sizing problem for CMOS circuits using convex optimization," IEEE Trans. on Computer-Aided Design, vol. 12, pp. 1621-1634, Nov 1993.

[10] V. Sundararajan, S. S. Sapatnekar, and K. K. Parhi, "Fast and Exact Transistor Sizing Based on Iterative Relaxation," IEEE Trans. on Computer-Aided Design, vol. 21, no. 5, pp. 568-581, May 2002.

[11] C. P. Chen, C. C. N. Chu, and D.F. Wong, "Fast and Exact Simultaneous Gate and Wire Sizing by Lagrangian Relaxation," Proc. Int'l Conf. on Computer-Aided Design, pp. 617- 624, Nov 1998.

[12] H Tennakoon, and C. Sechen, "Gate sizing using Lagrangian relaxation combined with a fast gradient-based pre-processing step,” Proc. Int'l. Conf. on Computer-Aided Design, pp. 395-402, Nov 2002.

[13] A. R. Conn, P. K. Coulman, R. A. Harring, G. L. Morril, C. Visweshwariah, and C. W. Wu, "JiffyTune: Circuit optimization using time-domain sensitivities," IEEE Trans. on Computer-Aided Design, vol. 17, no. 12, pp. 12921309, Dec 1998.

[14] A. R. Conn, I. M . Elfadel, W. W. Molzen, Jr, P. R. O’Brien, P. N. Strenski, C. Visweswariah, and C. B. Whan, "Gradient-Based optimization of custom circuits using static-timing formulation," Proc. Des. Auto. Con. (DAC) pp. 452-459, June 1999.

[15] Ivan E. Sutherland, Robert F. Sproull, David Harris, Logical Effort: Designing Fast CMOS Circuits, First Edition, Morgan Kaufmann, 1999.

[16] J.E. Dennis, D. M. Gay, and R. E. Welsch, "An adaptive nonlinear least-squares algorithm," ACM Trans. on Math. Software 7,3 Sept. 1981.

[17] Jorge Nocedal, and Stephen J. Wright, Numerical Optimization, Springer-Verlag, 1999. 
[18] M. S. Bazaraa, H. D. Sherali, C. M. Shetty, Nonlinear Programming: Theory and Algorithms, Second Edition, John Wiley and Sons, 1993 


\title{
Freeze: Engineering a Fast Repeater Insertion Solver for
} Power Minimization Using the Ellipsoid Method

\author{
Yuantao Peng, Xun Liu \\ Department of Electrical and Computer Engineering, \\ North Carolina State University, Raleigh, NC
}

\begin{abstract}
This paper presents a novel repeater insertion algorithm for the power minimization of realistic interconnect trees under given timing budgets. Our algorithm judiciously combines a local optimizer based on the dynamic programming technique and a global search engine using the ellipsoid method. As a result, our approach is capable of producing high-quality solutions at a very fast speed. Furthermore, our scheme is robust and does not need any manual tuning of the iteration-control parameters. We have developed a repeater insertion tool, called FREEZE, using the proposed algorithm and applied it to various interconnect trees with different timing targets. Experimental results demonstrate the high effectiveness of our approach. In comparison with the state-of-the-art low-power repeater insertion schemes, FREEZE requires 5.8 times fewer iterations on the average, achieving up to 27 times speedup with even better power savings. When compared with a dynamic programming based scheme, which guarantees the optimal solution, our tool delivers up to 50 times speedup with $0.9 \%$ power increase on the average.
\end{abstract}

Keywords: Interconnect, Repeater Insertion, Low Power

\section{REFERENCES}

[1] C. Alpert, A. Devgan, and S. T. Quay. Buffer insertion for noise and delay optimization. IEEE Trans. ComputerAided Design of Integrated Circuits and Systems, 18(11):1633-1645, Nov. 1999.

[2] C. J. Alpert and A. Devgan. Wire segmenting for improved buffer insertion. In Design Automation Conference, June 1997.

[3] C. J. Alpert, A. Devgan, and S. T. Quay. Buffer insertion with accurate gate and interconnect delay computation. In Design Automation Conference, June 1999.

[4] C. J. Alpert, J. Hu, S. S. Sapatnekar, and P. G. Villarrubia. A practical methodology for early buffer and wire resource allocation. IEEE Trans. CAD, 22(5), May 2003.

[5] H. B. Bakoglu. Circuits, Interconnects, and Packaging for VLSI. Reading, MA: Addison-Wesley, 1990.

[6] K. Banerjee and A. Mehrotra. A power-optimal repeater insertion methodology for global interconnects in nanometer designs. IEEE Trans. VLSI Systems, 49(11):2001-2007, Nov. 2002.

[7] C. P. Chen and N. Menezes. Noise-aware repeater insertion and wire sizing for on-chip interconnect using hierarchical moment-matching. In Design Automation Conference, June 1999.

[8] C.-C. N. Chu and D. F.Wong. Closed form solution to simultaneous buffer insertion/sizing and wire sizing. In Inter. Symp. on Physical Design, Apr. 1997.

[9] J. Cong, L. He, C. K. Koh, and P. H. Madden. Performance optimization of VLSI interconnect layout. Integration, the VLSI Journal, 21(1):1-94, Jan. 1996.

[10] G. S. Garcea, N. P. van der Meijs, and R. H. Otten. Simultaneous analytical area and power optimization for repeater insertion. In Inter. Conf. on CAD, Nov. 2003.

[11] L. P. P. P. van Ginneken. Buffer placement in distributed RC-tree networks for minimal Elmore delay. In Proc. Intl. Symposium on Circuits and Systems, 1990.

[12] N. Hedenstierna and K. O. Jeppson. CMOS circuit speed and buffer optimization. IEEE Trans. CAD, 6(2):270280, Feb. 1987.

[13] P. Kapur, G. Chandra, and K. C. Saraswat. Power estimation in global interconnect and its reduction using a novel repeater optimization methodology. In Design Automation Conference, June 2002.

[14] J. Lillis, C. K. Cheng, and T.-T. Y. Lin. Optimal wire sizing and buffer insertion for low power and a generalized delay model. J. of Solid-State Circuits, 31(3):437-447, Mar. 1996.

[15] I.-M. Liu, A. Aziz, and D. F. Wong. Meeting delay constraints in DSM by minimal repeater insertion. In Proc. IEEE Int. Conf. Design, Automation and Test Eur., Mar. 2000. 
[16] X. Liu, Y. Peng, and M. C. Papaefthymiou. Practical repeater insertion for low power: What repeater library do we need? In Design Automation Conference, June 2004.

[17] X. Liu, Y. Peng, and M. C. Papaefthymiou. RIP: An efficient hybrid repeater insertion scheme for low power. In Design, Automation, and Test in Europe, Mar. 2005.

[18] A. Nalamalpu and W. P. Burleson. A practical approach to DSM repeater insertion: Satisfying delay constraints while minimizing area and power. In IEEE International ASIC/SOC Conference, Sept. 2001.

[19] M. Nekili and Y. Savaria. Optimal methods of driving interconnections in VLSI circuits. In International Symposium on Circuits and Systems, May 1993.

[20] A. Nemirovsky and D. Yudin. Informational complexity and efficient methods for solution of convex extremal problems. J. Wiley \& Sons, New York, 1983.

[21] R. Otten. Global wires harmful? In Inter. Symp. on Physical Design, Apr. 1998.

[22] J. F. Shapiro. Mathematical Programming: Structures and Algorithms. Wiley-Interscience Publication, 1979.

[23] D. Sylvester and K. Keutzer. Getting to the bottom of deep submicron. In Inter. Conf. on CAD, Nov. 1998.

[24] D. Sylvester and K. Keutzer. A global wiring paradigm for deep submicron design. IEEE Trans. CAD, 19(2):242-252, Feb. 2000. 


\title{
Minimising Buffer Requirements of Synchronous Dataflow Graphs with Model Checking
}

\author{
Marc Geilen, Twan Basten and Sander Stuijk \\ Eindhoven University of Technology, Department of Electrical Engineering
}

\begin{abstract}
Signal processing and multimedia applications are often implemented on resource constrained embedded systems. It is therefore important to find implementations that use as little resources as possible. These applications are frequently specified as synchronous dataow graphs. Communication between actors of these graphs requires storage capacity. In this paper, we present an exact method to determine the minimum storage capacity required to execute the graph using model-checking techniques. This can be done for different measures of storage capacity. The problem is known to be NP-complete and because of this, existing buffer minimisation techniques are heuristics and hence not exact. Modern model-checking tools are quite efficient and they have been successfully applied to scheduling-related problems. We study the feasibility of this approach with examples.
\end{abstract}

Keywords: Synchronous Dataow, buffering, model-checking, optimization

\section{REFERENCES}

[1] M. Adé, R. Lauwereins, and J. A. Peperstraete. Data memory minimisation for synchronous data flow graphs emulated on dsp-fpga targets. In Proc. 34th DAC, pages 64-69, 1997. ACM Press.

[2] K. Altisen, G. Gößler, and J. Sifakis. A methodology for the construction of scheduled systems. In Proc. FTRTFT 2000, pages 106-120. Springer, 2000.

[3] T. Amnell, E. Fersman, L. Mokrushin, P. Pettersson, and W. Yi. Times: a tool for schedulability analysis and code generation of real-time systems. In Proc. of FORMATS'03, number 2791 in LNCS, pages 60-72. SpringerVerlag, 2004.

[4] S.S. Bhattacharyya, P.K. Murthy, and E.A. Lee. Software Synthesis from Dataflow Graphs. Kluwer, 1996.

[5] S.S. Bhattacharyya, P.K. Murthy, E.A. Lee. Synthesis of embedded software from synchronous dataow specifications. VLSI Sig. Proc. Syst., 21(2):151-166, 1999.

[6] J.T. Buck. Scheduling Dynamic Dataow Graphs with Bounded Memory Using the Token Flow Model. PhD thesis, University of California, EECS Dept., Berkeley, CA, 1993.

[7] S. Goddard and K. Jeffray. Managing memory requirements in the synthesis of real-time systems from processing graphs. In Proc. 4th IEEE Real-Time Technology and Applications Symp., June 1998, pages 59-70, 1998.

[8] R. Govindarajan, G.R. Gao, and P. Desai. Minimizing buffer requirements under rate-optimal schedule in regular dataflow networks. J. of VLSI Sig. Proc., 31:207-229, 2002.

[9] G.J. Holzmann. The SPIN Model Checker: Primer and Reference Manual. Addison Wesley, 2004.

[10] E.A. Lee and D.G. Messerschmitt. Synchronous data flow. IEEE Proceedings, 75(9):1235-1245, September 1987.

[11] P. K. Murthy. Scheduling Techniques for Synchronous and Multidimensional Synchronous Dataflow. PhD thesis, University of California, Berkeley, December 1996.

[12] P. K. Murthy and S. S. Bhattacharyya. Shared memory implementations of synchronous dataflow specifications. In Proc. DATE, March 2000. Paris, France, pages 404-410. IEEE Computer Society Press, 2000.

[13] H. Oh and S. Ha. Efficient code synthesis from extended dataflow graphs for multimedia applications. In Proc. $39^{\text {th }}$ DAC, 2002, New Orleans, LA, USA, pages 275-280. IEEE Computer Society, June 2002.

[14] P. Poplavko, T. Basten, M. Bekooij, J. van Meerbergen, and B. Mesman. Task-level timing models for guaranteed performance in multiprocessor networks-on-chip. In Proc. of CASES 2003, pages 63 - 72, IEEE CS Press , 2003.

[15] A. Roychoudhury, T. Mitra, and S. R. Karri. Formal verification of a system-on-chip bus protocol. In Proc. of DATE 2003, Munich, Germany, IEEE CS Press, 2003.

[16] S.K. Shukla and R.K. Gupta. A model checking approach to evaluating system level dynamic power management policies for embedded systems. In Proc. HLDVT'01, pages 53-57. IEEE Comp. Soc., 2001.

[17] W. Sung, J. Kim, and S. Ha. Memory efficient software synthesis from dataflow graph. In Proc. ISSS '98, pages 137-144. IEEE Computer Society, 1998. 
[18] M. Čubrić and P. Panangaden. Minimal memory schedules for dataflow networks. In Proc. CONCUR'93, LNCS Vol. 715, Springer, 1993. 


\title{
Unified High-Level Synthesis and Module Placement for Defect-Tolerant Microfluidic Biochips
}

\author{
Fei Su and Krishnendu Chakrabarty
}

Department of Electrical and Computer Engineering, Duke University, Durham, NC, USA

\begin{abstract}
Microfluidic biochips promise to revolutionize biosensing and clinical diagnostics. As more bioassays are executed concurrently on a biochip, system integration and design complexity are expected to increase dramatically. This problem is also identified by the 2003 ITRS document as a major system-level design challenge beyond 2009. We focus here on the automated design of droplet-based microfluidic biochips. We present a synthesis methodology that unifies operation scheduling, resource binding, and module placement for such "digital" biochips. The proposed technique, which is based on parallel recombinative simulated annealing, can also be used after fabrication to bypass defective cells in the microfluidic array. A real-life protein assay is used to evaluate the synthesis methodology.
\end{abstract}

Keywords: Synthesis, placement, defect tolerance, microfluidics, biochip

\section{REFERENCES}

[1] R. B. Fair et al., "Electrowetting-based on-chip sample processing for integrated microfluidics", Proc. IEDM, pp. 32.5.1-32.5.4, 2003.

[2] International Technology Roadmap for Semiconductors (ITRS), http://public.itrs.net/Files/2003ITRS/ Home2003.htm.

[3] J. Zeng and T. Korsmeyer, "Principles of droplet electrohydrodynamics for lab-on-a-chip", Lab on a Chip, pp. 265-277, 2004.

[4] F. Su and K. Chakrabarty, "Architectural-level synthesis of digital microfluidics-based biochips", Proc. ICCAD, pp. 223-228, 2004.

[5] G. De Micheli, Synthesis and Optimization of Digital Circuits. New York: McGraw-Hill, 1994.

[6] W. E. Dougherty and D. E. Thomas, "Unifying behavioral synthesis and physical design", Proc. DAC, pp. 756$760,2000$.

[7] K. Bazargan et al, "Integrating scheduling and physical design into a coherent compilation cycle for reconfigurable computing architectures", Proc. DAC, pp. 635-640, 2001.

[8] I. Koren and A. Singh, "Fault tolerance in VLSI circuits", IEEE Computer, vol. 23, pp. 73-83, 1990.

[9] G. Li and N. Aluru, "Efficient mixed-domain analysis of electrostatic MEMS", IEEE Trans. CAD, vol. 22, pp. 1228-1242, 2003.

[10] T. Mukherjee and G. K. Fedder, "Design methodology for mixeddomain systems-on-a-chip [MEMS design]", Proc. IEEE VLSI System Level Design, pp. 96-101, 1998.

[11] S. K. Tewksbury, "Challenges facing practical DFT for MEMS”, Proc. Defect and Tolerance in VLSI Systems, pp. 11-17, 2001.

[12] T. Zhang et al., Microelectrofluidic Systems: Modeling and Simulation, CRC Press, Boca Raton, FL, 2002.

[13] V. Srinivasan et al., "Protein stamping for MALDI mass spectrometry using an electrowetting-based microfluidic platform", Proc. SPIE, vol. 5591, pp. 26-32, 2004.

[14] M. Garey and D. Johnson, Computers and Intractability-a Guide to the Theory of NP-Completeness, Freeman, New York, 1979.

[15] S. W. Mahfoud and D. E. Goldberg, "Parallel recombinative simulated annealing: a genetic algorithm," Parallel Computing, vol. 21, pp. 1-28, 1995.

[16] J. C. Bean, "Genetics and random keys for sequencing and optimization", ORSA J. Computing, vol. 6, pp. 154160, 1994. 


\title{
Towards Scalable Flow and Context Sensitive Pointer Analysis
}

\section{Jianwen Zhu}

Department of Electrical and Computer Engineering,
University of Toronto, Toronto, Ontario, Canada

\begin{abstract}
Pointer analysis, a classic problem in software program analysis, has emerged as an important problem to solve in design automation, at a time when complex designs, specified in the form of $\mathrm{C}$ code, need to be synthesized or verified. However, precise pointer analysis algorithms that are both context and flow sensitive (FSCS), have not been shown to scale. In this paper, we report a new solution for FSCS analysis, which can evaluate the program states of all program points under billions of different calling paths. Our solution extends the recently proposed symbolic pointer analysis (SPA) technology, which exploits the efficiency of Binary Decision Diagrams (BDDs). With our new strategy of problem solving, called superposed symbolic computation, and its application on our generic pointer analysis framework, we are able to report the first result on all SPEC2000 benchmarks that completes context sensitive, flow insensitive analysis in seconds, and context sensitive, flow sensitive analysis in minutes.
\end{abstract}

Keywords: Pointer analysis, binary decision diagrams, High-level synthesis

\section{REFERENCES}

[1] M. Berndl, O. Lhoták, F. Qian, L. Hendren, and N. Umanee. Point-to analysis using BDD. In Proceedings of the ACM SIGPLAN Conference on Programming Language Design and Implementation, San Diego, June 2003.

[2] R. E. Bryant. Graph-based algorithms for Boolean function manipulation. IEEE Transactions on Computer, C35(8):677-691, August 1986.

[3] J. R. Burch, E. M. Clarke, K. L. McMillan, D. L. Dill, and L. J. Hwang. Symbolic model checking: 1020 states and beyond. In Proceedings of the Fifth Annual IEEE Symposium on Logic in Computer Science, Washington, DC, 1990.

[4] V. T. Chakaravarthy. New results on the computability and complexity of points-to analysis. In Proceedings of Priciple of Programming Languages (POPL'03), January 2003.

[5] B.-C. Cheng and W.-M. W. Hwu. Modular interprocedural pointer analysis using access paths: Design implementation and evaluation. In Proceedings of SIGPLAN Conference on Programming Language Design and Implementation, pages 57-69, Vancouver, British Columbia, Canada, June 2000.

[6] O. Coudert, C. Berthet, and J. C. Madre. A unified framework for the formal verification of sequential circuits. In Proceedings of the International Conference on Computer-Aided Design, pages 126-129, November 1990.

[7] R. Cytron, J. Ferrante, B. K. Rosen, M. N. Wegman, and F. K. Zadeck. Efficiently computing static single assignment form and the control dependence graph. ACM Trans. Prog. Lang. Syst., 13(4), October 1991.

[8] S. A. Edwards. The challenges of hardware synthesis from C-like languages. In Proceedings of the International Workshop of Logic and Synthesis (IWLS), June 2004.

[9] M. Emami, R. Ghiya, and L. J. Hendren. Context-sensitive interprocedural points-to analysis in the presence of function pointers. In Proceedings of SIGPLAN Conference on Programming Language Design and Implementation, pages 242-256, 1994.

[10] M. Fähndrich, J. Rehof, and M. Das. Scalable context-sensitive flow analysis using instantiation constraints. In Proceedings of the ACM SIGPLAN Conference on Programming Language Design and Implementation, pages 253263, Vancouver, British Columbia, Canada, June 2000.

[11] J. S. Foster, M. Fähndrich, and A. Aiken. Polymorphic versus monomorphic flow-insensitive points-to analysis for C. In Proceedings of Static Analysis Symposium, pages 175-198, June 2000.

[12] D. Gajski, J. Zhu, D. Doemer, A. Gerstlauer, and S. Zhao. SpecC: Specification Language and Methodology. Kluwer Academic Publishers, Boston, March 2000.

[13] N. Heintze and O. Tardieu. Ultra-fast aliasing analysis using CLA: A million lines of C code in a second. In Proceedings of SIGPLAN Conference on Programming Language Design and Implementation, pages 254-263, 2001.

[14] M. Hind. Pointer analysis: Haven't we solved this problem yet. In ACM SIGPLAN-SIGSOFT Workshop on Program Analysis for Software Tools and Engineering (PASTE), June 2001. 
[15] O. Lhoták and L. Hendren. Jedd: A BDD-based relational extension of Java. In Proceedings of SIGPLAN Conference on Programming Language Design and Implementation, June 2004.

[16] D. Liang and M. J. Harrold. Efficient computation of parameterized pointer information for interprocedural analyses. In Proceedings of Static Analysis Symposium, pages 279-298, 2001.

[17] S. Liao, S. Tjiang, and R. Gupta. An efficient implementation of reactivity for modeling hardware in the Scenic design environment. In Proceeding of the $34^{\text {th }}$ Design Automation Conference, 1997.

[18] G. D. Micheli. Hardware synthesis from C/C++ models. In Proceedings of the Design Automation and Test Conference in Europe, March 1999.

[19] P. Panda, L. Semeria, and G. D. Micheli. Cache-efficient memory layout of aggregate data structures. In Proceedings of the International Symposium on System Synthesis, September 2001.

[20] E. Rieffel and W. Polak. An introduction to quantum computing for non-physicists. ACM Computing Surveys, (32(2)), September 1997.

[21] L. Semeria and G. D. Micheli. Resolution, optimization, and encoding of pointer variables for the behavioral synthesis from C. IEEE Transactions on Computer-Aided Design of Integrated Circuits and Systems, February 2001.

[22] L. Semeria, K. Sata, and G. D. Micheli. Synthesis of hardware models in C with pointers and complex data structures. IEEE Transactions on Computer-Aided Design of Integrated Circuits and Systems, February 2001.

[23] F. Somenzi. CUDD: Binary decision diagram package release. http://vlsi.Colorado.EDU/fabio/CUDD/ cuddIntro.html, 1998.

[24] SystemC Web Site. http://www.systemc.org.

[25] J. Whaley and M. Lam. Cloning-based context-sensitive pointer alias analysis using binary decision diagrams. In Proceedings of SIGPLAN Conference on Programming Language Design and Implementation, June 2004.

[26] R. Wilson and M. Lam. Efficient context-sensitive pointer analysis for C programs. In Proceedings of the ACM SIGPLAN Conference on Programming Language Design and Implementation, pages 1-12, June 1995.

[27] J. Zhu. Static memory allocation by pointer analysis and coloring. In Design Automation and Test in Europe, March 2001.

[28] J. Zhu. Symbolic pointer analysis. In Proceedings of the International Conference in Computer Aided Design, San Jose, November 2002.

[29] J. Zhu and S. Calman. Symbolic pointer analysis revisited. In Proceedings of SIGPLAN Conference on Programming Language Design and Implementation, June 2004.

[30] J. Zhu, R. Doemer, and D. Gajski. Syntax and semantics of SpecC+ language. In Proceedings of the Ninth Workshop on Synthesis and System Integration of Mixed Technologies, Japan, December 1997. 


\title{
MiniBit: Bit-Width Optimization via Affine Arithmetic
}

Dong-U Lee, Altaf Abdul Gaffar, Oskar Mencer and Wayne Luk

\author{
Department of Computing, Imperial College, London, United Kingdom
}

\begin{abstract}
MiniBit, our automated approach for optimizing bit-widths of fixed-point designs is based on static analysis via affine arithmetic. We describe methods to minimize both the integer and fraction parts of fixed-point signals with the aim of minimizing circuit area. Our range analysis technique identifies the number of integer bits required. For precision analysis, we employ a semi-analytical approach with analytical error models in conjunction with adaptive simulated annealing to find the optimum number of fraction bits. Improvements for a given design reduce area and latency by up to $20 \%$ and $12 \%$ respectively, over optimum uniform fraction bit-widths on a Xilinx Virtex-4 FPGA.

Keywords: Affine Arithmetic, Bit-Width, Fixed-Point, FPGA, Simulated Annealing

\section{REFERENCES}

[1] A. Abdul Gaffar, O. Mencer, W. Luk, and P. Cheung. Unifying bit-width optimisation for fixed-point and floating-point designs. In Proc. IEEE Symp. Field-Programmable Custom Computing Machines, pages 79-88, 2004.

[2] R. Cmar, L. Rijnders, P. Schaumont, S. Vernalde, and I. Bolsens. A methodology and design environment for DSP ASIC fixed point refinement. In Proc. ACM/IEEE Design Automation and Test in Europe Conf., pages 271276, 1999.

[3] C. Fang, R. Rutenbar, and T. Chen. Fast, accurate static analysis for fixed-point finite-precision effects in DSP designs. In Proc. ACM/IEEE Int'l Conf. on Computer-Aided Design, pages 275-282, 2003.

[4] C. Fang, R. Rutenbar, M. P"uschel, and T. Chen. Toward efficient static analysis of finite-precision effects in DSP applications via affine arithmetic modeling. In Proc. ACM/IEEE Design Automation Conf., pages 496-501, 2003.

[5] L. Ingber. Adaptive Simulated Annealing (ASA) 25.15, 2004. http://www.ingber.com/\#ASA.

[6] L. de Figueiredo and J. Stolfi. Self-validated numerical methods and applications. In Brazilian Mathematics Colloquium monograph. IMPA, Brazil, 1997.

[7] D. Menard and O. Sentieys. Automatic evaluation of the accuracy of fixed-point algorithms. In Proc. ACM/IEEE Design Automation and Test in Europe Conf., pages 1530-1591, 2002.

[8] O. Mencer, D. Pearce, L. Howes, and W. Luk. Design space exploration with A Stream Compiler. In Proc. IEEE Int'l Conf. Field-Programmable Technology, pages 270-277, 2003.
\end{abstract}




\title{
A Non-Parametric Approach for Dynamic Range Estimation of Nonlinear Systems
}

Bin Wu, Jianwen Zhu, Farid N. Najm

Department of Electrical and Computer Engineering, University of Toronto, Toronto, Ontario, Canada

\begin{abstract}
It has been widely recognized that the dynamic range information of an application can be exploited to reduce the datapath bitwidth of either processors or ASICs, and therefore the overall circuit area, delay, and power consumption. Recent advances in analytical dynamic range estimation methods indicate that by systematically decomposing the system inputs into orthonormal random variables using a mathematical procedure called polynomial chaos expansion (PCE), output statistics of interest can be obtained for both linear and nonlinear systems. Despite its power for capturing both spatial and temporal correlation, the application of this method has been limited only to near-Gaussian inputs. In this paper, we propose the first algorithm with the capacity of handling both near-Gaussian and non-Gaussian input signals. Our method is based on the use of independent component analysis (ICA). Our experiments show that the new algorithm can reduce the original relative errors of 2 nd order moments from $25 \%-$ $65 \%$ to $1 \%-2 \%$.
\end{abstract}

Keywords: Dynamic range estimation, Non-Gaussian, Nonlinear, Independent component analysis, Non-parametric

\section{REFERENCES}

[1] B. Wu, J. Zhu, and F. N. Najm. Dynamic range estimation for nonlinear systems. In ACM/IEEE International Conference on Computer-Aided Design (ICCAD-04), San Jose, CA, November 7-11 2004.

[2] R. G. Ghanem and P. D. Spanos. Stochastic Finite Elements: A Spectral Approach. Dover Publications, Inc., Mineola, NY, revised edition, 2003.

[3] R. G. Ghanem. The nonlinear gaussian spectrum of lognormal stochastic processes and variables. ASME Journal of Applied Mechanics, 66(4):964-973, 1999.

[4] A. Hyvarinen, J. Karhunen, and E. Oja. Independent Component Analysis. John Wiley \& Sons, New York, 2001.

[5] A. Hyvarinen. Fast and robust fixed-point algorithms for independent component analysis. IEEE Transactions on Neural Networks, 10(3):626-634, 1999. 


\title{
Path Delay Test Compaction with Process Variation Tolerance
}

\author{
Seiji Kajiharal, Masayasu Fukunaga ${ }^{1}$, Xiaoqing Wen $^{1}$, Toshiyuki Maeda ${ }^{2}$, \\ Shuji Hamada ${ }^{2}$, Yasuo Sato ${ }^{2}$ \\ ${ }^{1}$ Kyushu Institute of Technology, 680-4 Kawazu, Iizuka, Japan \\ ${ }^{2}$ Semiconductor Technology Academic Research Center, 3-17-2 Shinyokohama, Kita-ku, \\ Yokohama, Japan
}

\begin{abstract}
In this paper we propose a test compaction method for path delay faults in a logic circuit. The method generates a compact set of two-pattern tests for faults on long paths selected with a criterion. While the proposed method generates each two-pattern test for more than one fault in the target fault list as well as ordinary test compaction methods, secondary target faults are selected from the fault list such that many other faults, which may not be included in the fault list, are detected by the test pattern. Even if faults on long paths in a manufactured circuit are not included in the fault list due to a process variation or noise, the compact test set would detect the longer untargeted faults, i.e., the test set has a noise or variation tolerant nature. Experimental results show that the proposed method can generate a compact test set and it detects longer untargeted path delay faults efficiently.
\end{abstract}

Keywords: delay testing, test compaction, path delay fault, process variation

\section{REFERENCES}

[1] M. L. Bushnell, and V. D. Agrawal, Essentials of Electronic Testing for Digital, Memory \& Mixed-Signal VLSI Circuits, Kluwer Academic Publishers, 2000.

[2] G. L. Smith, "Model for delay faults based upon paths," Int'l Test Conf., pp.342-349, 1985.

[3] Z.Barzilai and B.K.Rosen, "Comparison of AC Self-testing Procedures," Int'l Test Conf., pp.89-01, 1983.

[4] W.-N.Li, S.M.Reddy, S.K.Sahni, "On Path Selection in Combinational Logic Circuits," IEEE Trans. on CAD., vol.8, pp.56-63, 1989

[5] A. Murakami, S. Kajihara, T. Sasao, I. Pomeranz, and S. M. Reddy, "Selection of Potentially Testable Path Delay Faults for Test Generation," Int'l Test Conf., pp. 376-384, 2000.

[6] M. Sharma and J. H. Patel, "Finding a Small Set of Longest Testable Paths that Cover Every Gate," Int'l Test Conf., pp. 974-982, Oct. 2002.

[7] Y. Shao, S. M. Reddy, I. Pomeranz, S. Kajihara, "On Selecting Paths to Test in Scan Designs," Journal of Electronic Testing Theory and Applications, volume 19, pp. 447-456, August 2003.

[8] W. Qiu and D. M. H. Walker, "An Efficient Algorithm for Finding the K Longest Testable Paths Through Each Gate in a Combinational Circuit," Int'l Test Conf., pp. 592-601, Sept. 2003.

[9] L.-C. Chen, S. K. Gupta and M. A. Breuer, "High Quality Robust Tests for Path Delay Faults," VLSI Test Symp., pp. 88-93, April 1997.

[10] K-T Cheng, S. Dey, M. Rodgers, K. Roy. "Test Challenges for Deep Sub-Micron Technologies," Design Automation Conf., pp.142-149, June 2000.

[11] J.-J. Liou, A. Krstic, Y.-M. Jiang and K.-T. Cheng, "Path Selection and Pattern Generation for Dynamic Timing Analysis Considering Power Supply Noise Effects," Intl. Conf. on Computer-Aided Design, pp. 493-496, Nov. 2000.

[12] J.-J. Liou, A. Krstic, L.-C. Wang, K.-T. Cheng. "False-Path-Aware Statistical Timing Analysis and Efficient

Path Selection for Delay Testing and Timing Validation," Design Automation Conf., pp.566-569, 2002.

[13] S. Tragoudas, S. Padmanaban, “A Critical Path Selection Method for Delay Testing," Int'l Test Conf., pp. 232241 , Oct. 2004.

[14] I. Pomeranz and S. M. Reddy, "Test Enrichment for Path Delay Faults Using Multiple Sets of Target Faults," Conf. on Design Automation and Test in Europe, pp. 722-729, March 2002.

[15] I. Pomeranz and S. M. Reddy, "A Postprocessing Procedure of Test Enrichment for Path Delay Faults," Asian Test Symposium, pp. 448-453, Nov. 2004.

[16] S. Bose, P. Agrawal, V. Agrawal, "Generation of compact delay tests by multiple path activation," Int'l Test Conf., pp. 714-723, Oct. 1993.

[17] J. Saxena; D.K.Pradhan, "A method to derive compact test sets for path delay faults in combinational circuits," Int'l Test Conf., pp. 724-733, Oct. 1993. 
[18] S.Kajihara, K.Kinoshita, I.Pomeranz, S.M.Reddy, "A Method for Identifying Robust Dependent and Functionally Unsensitizable Paths," Int'l Conf. on VLSI Design, pp.82-87, 1997.

[19] Z.Li, Y.Min, R.K.Brayton, "Efficient Identification of Non-Robustly Untestable Path Delay Faults," Int'l Test Conf., pp.992-997, 1997.

[20] K.Heragu, J.H.Patel, V.D.Agrawal, "Fast Identification of Untestable Delay Faults Using Implications," Intl. Conf. on Computer-Aided Design, pp.642-647, 1997.

[21] P. Goel and B. C. Rosales, "Test Generation \& Dynamic Compaction of Tests," in Digest of Papers 1979 Test Conf. , pp. 189-192, Oct. 1979.

[22] I. Hamzaoglu, J.H. Patel, "Compact two-pattern test set generation for combinational and full scan circuits," Int'l Test Conf., pp. 944-953, Oct. 1998.

[23] S. Kajihara, I. Pomeranz, K. Kinoshita and S. M. Reddy, "Cost-Effective Generation of Minimal Test Sets for Stuck-at Faults in Combinational Logic Circuits," IEEE Trans. Computer-Aided Design of Integrated Circuits and Systems, Vol. 14, No. 12, pp.1496-1504, Dec. 1995.

[24] I. Hamzaoglu and J. H. Patel, "Test Set Compaction Algorithms for Combinational Circuits," Intl. Conf. on Computer-Aided Design, pp. 283-289, Oct. 1998.

[25] M. Abramovici, M. A. Breuer, A. D. Friedman, Digital Systems Testing and Testable Design, Piscataway, New Jersey: IEEE Press, 1990. 


\title{
A DFT Approach for Diagnosis and Process Variation-Aware Structural Test of Thermometer Coded Current Steering DACs
}

\author{
Rasit Onur Topaloglu and Alex Orailoglu \\ University of California San Diego, Computer Science and Engineering Department \\ La Jolla, CA
}

\begin{abstract}
A design for test (DFT) hardware is proposed to increase the controllability of a thermometer coded current steering digital to analog converter. A procedure is introduced to reduce the diagnosis and structural test time from quadratic to linear using the proposed DFT hardware. To evaluate the applicability of the proposed technique, principal component analysis is used to create virtual process variations to simulate in lieu of semiconductor fabrication data. An architecture specific soft fault model is suggested for the diagnosis problem. Random errors according to the fault model are introduced in the virtual test environment on top of the process variations and it is shown that diagnosis of a fault is possible with high accuracy with the proposed method. The same technique employing principal component analysis is furthermore used to provide process variation-aware reference test comparison values for a structural test of the DAC. The structural test provides a mechanism to test for even unmodeled manufacturing faults. The process variation-aware test values help detect defects even under process variations. The proposed DFT hardware and method are low cost and quite suitable for a built-in self diagnosis and test implementation.
\end{abstract}

Keywords: DFT, BIST, CS-DAC, current steering, test, process variation-aware test, diagnosis

\section{REFERENCES}

[1] C. J. Abel, C. Michael, M. Ismail, C. S. Teng, and R. Lahri. Characterization of transistor mismatch for statistical cad of submicron cmos analog circuits. In ISCAS, pages 1401-1404, 1993.

[2] M. Albiol, J. L. Gonzales, and E. Alarcon. Mismatch and dynamic modeling of current sources in currentsteering cmos d/a converters: An extended design procedure. IEEE TCAS-I, 51(1):159-169, Jan. 2004.

[3] K. Arabi and B. Kaminska. Oscillation test strategy for analog and mixed-signal integrated circuits. In VTS, pages 476-482, 1996.

[4] S. Chakrabarty, V. Rajan, J. Ying, M. Mansjur, K. Pattipati, and S. Deb. A virtual test-bench for analog circuit testability analysis and fault diagnosis. In AUTOTESTCON, pages 337-352, 1998.

[5] K. Doris, A. van Roermund, and D. Leenaerts. Mismatch-based timing errors in current steering dacs. In ISCAS, pages I.977-I.980, 2003.

[6] P. G. Drennan and C. C. McAndrew. A comprehensive mosfet mismatch model. In IEDM, pages 167-170, 1999.

[7] F. J. Ferguson and J. P. Shen. A cmos fault extractor for inductive fault analysis. IEEE TCAD of Integrated Circuits and Systems, 7(11):1181-1194, Nov. 1988.

[8] J. Hou and A. Chatterjee. Concurrent transient fault simulation for analog circuits. IEEE TCAD, 22(10):13851398, Oct. 2003.

[9] L. Milor and A. Sangiovanni-Vincentelli. Optimal test set design for analog circuits. In IEEE ICCAD, pages 294-297, 1990.

[10] K. O'Sullivan, M. H. C. Gorman, and V. Callaghan. A 12-bit 320-msample/s current-steering cmos d/a converter in $0.44 \mathrm{~mm} 2$. IEEE JSSC, 39(7):1064-1072, July 2004.

[11] J. M. Pelgrom, C. J. Duinmaijer, and P. G. Welbers. Matching properties of mos transistors. IEEE JSSC, 24(5):1433-1439, Oct. 1989.

[12] E. J. Peralias, A. Rueda, and J. L. Huertas. Structural testing of pipelined analog to digital converters. In ISCAS, pages 436-439, 2001.

[13] J. Roh and J. A. Abraham. A comprehensive signature analysis scheme for oscillation-test. IEEE TCAD, 22(10):1409-1423, Oct. 2003.

[14] J. Savir and Z. Guo. On the detectability of parametric faults in analog circuits. In ICCD, pages 273-276, 2002.

[15] M. Slamani and B. Kaminska. Analog circuit fault diagnosis based on sensitivity computation and functional testing. IEEE Design and Test of Computers, 9(1):30-39, Mar. 1992. 
[16] M. Slamani and B. Kaminska. A cmos fault extractor for inductive fault analysis. IEEE TCAD, 9(1):30-39, Mar. 1992.

[17] J. A. Starzyk, R. P. Mohn, and J. Liang. A cost-effective approach to the design and layout of a 14-b currentsteering dac macrocell. IEEE TCAS-I, 51(1):196-200, Jan. 2004.

[18] S. Sunter and N. Nagi. Test metrics for analog parametric faults. In VTS, pages 226-234, 1999.

[19] Q. Zhang, J. J. Liou, J. R. McMacken, J. Thomson, and P. Layman. Spice modeling and quick estimation of mosfet mismatch based on bsim3 model and parametric tests. IEEE JSSC, 36(10):1592-1595, Oct. 2001. 


\title{
Resistive-Open Defect Injection in SRAM Core-Cell: Analysis and Comparison between $0.13 \mu \mathrm{m}$ and $90 \mathrm{~nm}$ Technologies
}

\author{
L. Dilillo*, P. Girard*, S. Pravossoudovitch*, A. Virazel*, M. Bastian** \\ *LIRMM - Université de Montpellier II / CNRS, Montpellier Cedex 5, France \\ **Infineon Technologies France, Sophia-Antipolis, France
}

\begin{abstract}
Resistive-open defects appear more and more frequently in VDSM technologies. In this paper we present a study concerning resistive-open defects in the core-cell of SRAM memories. The first target of this work is a comparison of the effect produced by resistive-open defects in the 0.13 $\mu \mathrm{m}$ and $90 \mathrm{~nm}$ core-cell. We show that the $90 \mathrm{~nm}$ core-cell is more robust than the $0.13 \mu \mathrm{m}$ corecell in presence of resistive-open defects. On the other hand we show that dynamic faults are most likely to occur in the $90 \mathrm{~nm}$ than in $0.13 \mu \mathrm{m}$ core-cell. Finally we propose a unique March test solution that ensures the complete coverage of all the extracted fault models for both technologies.
\end{abstract}

Keywords SRAM Memories, Core-cell, Dynamic Faults, March Test

\section{REFERENCES}

[1] Semiconductor Industry Association (SIA), "International Technology Roadmap for Semiconductors (ITRS)", 2003 Edition.

[2] A.J. van de Goor, "Using March Tests to Test SRAMs", IEEE Design \& Test of Computers, vol.10, n¹, Jun 1993, pp.8-14.

[3] R.D. Adams and E.S. Cooley, "Analysis of Deceptive Destructive Read Memory Fault Model and Recommended Testing”, IEEE North Atlantic Test Workshop, May 1996.

[4] K. Baker et al., "Defect-Based Delay Testing of Resistive Vias-Contacts. A Critical Evaluation", Proc. Int. Test Conference, 1999, pp. 467-476.

[5] C.-M. James et al., "Testing for Resistive Opens and Stuck Opens”, Proc. Int. Test Conference, 2001, pp. 10491058 .

[6] W. Needham et al., "High Volume Microprocessor Test Escapes - An Analysis of Defects Our Tests are Missing", Proc. Int. Test Conference, 1998, pp. 25-34.

[7] A.J. van de Goor and Z. Al-Ars, "Functional Memory Faults: A Formal Notation and a Taxonomy", Proc. IEEE VLSI Test Symposium, May 2000, pp.281-289.

[8] Z. Al-Ars and A.J. van de Goor, "Static and Dynamic Behavior of Memory Cell Array Opens and Shorts in Embedded DRAMs", Proc. Design, Automation and Test in Europe, 2001, pp. 496-503.

[9] S. Hamdioui, Z Al-Ars and A.J. van de Goor, "Testing Static and Dynamic Faults in Random Access Memories", Proc. IEEE VLSI Test Symposium, 2002, pp. 395-400.

[10] Borri, M. Hage-Hassan, P. Girard, S. Pravossoudovitch, A. Virazel, "Defect-Oriented Dynamic Fault Models for Embedded-SRAMs", Proc. IEEE European Test Workshop, 2003, pp. 23-28.

[11] L. Dilillo, P. Girard, S. Pravossoudovitch, A. Virazel, S. Borri, M. Hage-Hassan, "Dynamic Read Destructive Fault in Embedded-SRAMs: Analysis and March Test Solutions", Proc. IEEE European Test Symposium, 2004.

[12] D. Niggemeyer, M. Redeker and J. Otterstedt, "Integration of Non-classical Faults in Standard March Tests", Records of the IEEE Int. Workshop on Memory Technology, Design and Testing, 1998, pp. 91-96.

[13] M. Nicolaidis, "Theory of Transparent BIST for RAMs", IEEE Trans. On Computers, vol. 45, N 10, October 1996, pp. 1141-1155. 


\title{
Asynchronous Circuits Transient Faults Sensitivity Evaluation
}

\author{
Y. Monnet, M. Renaudin, R. Leveugle \\ TIMA Laboratory, GRENOBLE cedex-FRANCE
}

\begin{abstract}
This paper presents a transient faults sensitivity evaluation for Quasi Delay Insensitive (QDI) asynchronous circuits. Because of their specific architecture, asynchronous circuits have a very different behavior than synchronous circuits in the presence of faults. We address the effects of transient faults in QDI circuits and describe the causes that lead the faults to be memorized into one or more soft errors. Therefore, a refined fault sensitivity criterion is defined for this class of circuits. This methodology enables us to point out the weak parts of a circuit. An analysis tool is implemented to support this evaluation. This tool provides a quantitative study of the fault sensitivity, and enables us to compare the robustness of different architectures of a circuit along the steps of its design flow. The objective of this work is to evaluate the circuits robustness against natural faults (single fault model) and intentional fault injection (multiple faults model).

Keywords: Asynchronous circuits, Quasi Delay Insensitive, transient fault, fault model, simulation
\end{abstract}

\section{REFERENCES}

[1] R. Leveugle, K. Hadjiat, "Multi-level fault injections in VHDL descriptions: alternative approaches and experiments", Journal of Electronic Testing: Theory and Applications (JETTA), Kluwer, vol. 19, no. 5, October 2003, pp. 559-575.

[2] D. Alexandrescu, L. Anghel, M. Nicolaidis, "New methods for evaluating the impact of single event transients in VDSM ICs", The IEEE International Symposium on Defect and Fault Tolerance in VLSI Systems, Vancouver, Canada, November 6-8, 2002, IEEE Computer Society Press, Los Alamitos, California, 2002, pp. 99-107.

[3] M. Bellato, P. Bernardi, D. Bortolato, A. Candelori, M. Ceschia, A. Paccagnella, M. Rebaudengo, M. Sonza Reorda, M. Violante, P. Zambolin , "Evaluating the effects of SEUs affecting the configuration memory of an SRAM-based FPGA", Design Automation and Test in Europe Conference (DATE), February 16-20, 2004, pp. 584589.

[4] M. Sonza-Reorda, M. Violante, "Accurate and efficient analysis of single event transients in VLSI circuits", $9^{\text {th }}$ IEEE International On-Line Testing symposium, Kos, Greece, July 7-9, 2003, pp. 101-105.

[5] Y. Monnet, M. Renaudin, R. Leveugle, "Asynchronous circuits sensitivity to fault injection", 10th IEEE International On-Line Testing Symposium, Madeira Island, Portugal, July 12-14, 2004.

[6] M. Renaudin, "Asynchronous Circuits and Systems: a promising design alternative", MicroelectronicsEngineering Journal, Elsevier Science, Guest Editors: P.Senn, M. Renaudin, J. Boussey, Vol54, Nº1-2, 2000, pp.133-149.

[7] D. Alexandrescu, L. Anghel, M. Nicolaidis, "Simulating single event transients in DVSM ICs for ground level radiation", 3rd IEEE Latin American Test Workshop (LATW'02), Montevideo, Uruguay, February 10-13, 2002.

[8] E. Biham, A. Shamir, "Differential Fault Analysis of Secret Key Cryptosystems", Advances in Cryptology CRYPTO 1997, LNCS 1294, pp. 513-525, 1997.

[9] M. Renaudin, G. F. Bouesse, P. Proust, J-P Tual, L. Sourgen, F. Germain, "High-Security Smartcards", Design Automation and Test in Europe Conference (DATE), Feb 16-20, 2004.

[10] NIST, Data Encryption Standard (DES), FIPS PUB 46-2, National Institute of Standards and Technology. http://csrc.nist.gov/csrc/fedstandards.html

[11] C. LaFrieda, R.Manohar, "Fault Detection and Isolation Techniques for Quasi Delay-Insensitive Circuits", International Conference on Dependable Systems and Networks (DSN'04), Italy, June 28 - July 01, 2004, p.41

[12] Wonjin Jang, Alain J. Martin, "SEU-Tolerant QDI Circuits",11th IEEE International Symposium on Asynchronous Circuits and Systems, New York City, USA, March 13-16, 2005, pp. 156-165.

[13] Y. Monnet, M. Renaudin, R. Leveugle, "Hardening Techniques against Transient Faults for Asynchronous Circuits", 11th IEEE International On-Line Testing Symposium, Saint Raphael, France, July 6-8, 2005. 


\title{
Deterministic Approaches to Analog Performance Space Exploration (PSE)
}

\author{
Daniel Mueller, Guido Stehr, Helmut Graeb, Ulf Schlichtmann \\ Institute for Electronic Design Automation, Techn. Univ. Muenchen, Munich, Germany
}

\begin{abstract}
Performance space exploration (PSE) determines the range of feasible performance values of a circuit block for a given topology and technology. In this paper, we present two deterministic approaches for PSE. One approximates the feasible performance space based on linearized circuit models and is suitable for investigating a large number of performances. The other one computes discretizations of the Pareto front of competing performances. In addition, a motivation and application of PSE using a hierarchical design example is presented.
\end{abstract}

Keywords: Performance Space Exploration, Analog Integrated Circuits, Pareto Optimization, Fourier Motzkin Elimination

\section{REFERENCES}

[1] G. Stehr, H. Graeb and K. Antreich. Performance Trade-off Analysis of Analog Circuits By Normal-Boundary Intersection. ACM/IEEE DAC 2003.

[2] G. Stehr, H. Graeb and K. Antreich. Analog Performance Space Exploration by Fourier-Motzkin Elimination with Application to Hierarchical Sizing. IEEE ICCAD 2004.

[3] H. Chang, E. Charbon, U. Choudhury, A. Demir, E. Felt,E. Liu, E. Malavasi, A. Sangiovanni-Vincentelli, and I. Vassiliou. A Top-Down, Constraint-Driven Design Methodology for Analog Integrated Circuits. Kluwer Acad. Pub. 1997.

[4] I. Vassiliou, H. Chang, A. Demir, E. Charbon, P. Miliozzi and A. Sangiovanni-Vincentelli. A Video Driver System Designed Using a Top-Down, Constraint-Driven Methodology. IEEE ICCAD 1996.

[5] G.J. Gomez, S.H.K. Embabi, E. Sanchez-Sinencio and M.C. Levebvre. A nonlinear macromodel for CMOS OTAs. IEEE ISCAS 1995.

[6] F. De Bernardinis, M. I. Jordan, and A. Sangiovanni-Vincentelli. Support vector machines for analog circuit performance representation. ACM/IEEE DAC, 2003.

[7] M. del Mar Hershenson, S. P. Boyd, and T. H. Lee. Optimal design of a CMOS Op-Amp via geometric programming. IEEE Trans. CAD 2001.

[8] R. Harjani and J. Shao. Feasibility and performance region modeling of analog and digital circuits. Analog Integrated Circuits and Signal Processing 1996.

[9] B. D. Smedt and G. G. E. Gielen. WATSON: Design space boundary exploration and model generation for analog and RF IC design. IEEE Trans. CAD 2003.

[10] G. Van der Plas, G. Debyser, F. Leyn, K. Lampaert, J. Vandenbussche, G. Gielen, W. Sansen, P. Veselinovic, and D. Leenaerts. AMGIE.A synthesis environment for CMOS analog integrated circuits. IEEE Trans. CAD 2001.

[11] M. Lightner, T. Trick, and R. Zug. Circuit optimization and design. Circuit Analysis, Simulation and Design, Part 2 (A. Ruehli). Advances in CAD for VLSI 3, 1987.

[12] G. Stehr, M. Pronath, F. Schenkel, H. Graeb, and K. Antreich. Initial sizing of analog integrated circuits by centering within topology-given implicit specifications. IEEE ICCAD 2003.

[13] A. Fuad Mas'ud, T. Ohtsuka, and H. Kunieda. Translation of specifications in hierarchical analog LSI design. In IEEE ISCAS 1996.

[14] W. Nye, D. Riley, A. Sangiovanni-Vincentelli, and A. Tits. DELIGHT.SPICE: An optimization-based system for the design of integrated circuits. IEEE Trans. CAD 1988.

[15] R. Phelps, M. Krasnicki, R. Rutenbar, L.R. Carley, and J. Hellums. Anaconda: Simulation-based synthesis of analog circuits via stochastic pattern search. IEEE Trans. CAD 2000.

[16] H. Graeb, S. Zizala, J. Eckmueller, and K. Antreich. The sizing rules method for analog integrated circuit design. IEEE/ACM ICCAD 2001.

[17] A Somani, P P Chakrabarti and A Patra. Mixing global and local competition in genetic optimization-based design space exploration of analog circuits, DATE 2005.

[18] $\mathrm{T}$ Eeckelaert, $\mathrm{T}$ McConaghy and $\mathrm{G}$ Gielen. Efficient multiobjective synthesis of analog circuits using hierarchical Pareto-optimal hypersurfaces, DATE 2005.

[19] Maria del Mar Hershenson. Efficient Description of the Design Space of Analog Circuits. ACM/IEEE DAC 2003. 
[20] M. Vogels, G. Gielen. Architectural Selection of A/D Converters. ACM/IEEE DAC 2003.

[21] Hongzhou Liu, Amit Singhee, Rob A. Rutenbar, L. Richard Carley. Remembrance of Circuits Past: Macromodeling by Data Mining in Large Analog Design Spaces. ACM/IEEE DAC 2002.

[22] J. Vandenbussche, K. Uyttenhove, E. Lauwers, M. Steyaert and G. Gielen. Systematic Design of a 200 Ms/s 8bit Interpolating/Averaging A/D Converter. ACM/IEEE DAC 2002.

[23] F De Bernardinis, S Gambini, F Vincis, F Svelto, R Castello, A Sangiovanni Vincentelli. Design Space Exploration for a UMTS Front-end Exploiting Analog Platforms. IEEE ICCAD 2004.

[24] Glenn A Wolfe, Ranga Vemuri. Adaptive Sampling and Modeling of Analog Circuit Performance Parameters with Pseudo-Cubic Splines. IEEE ICCAD 2004.

[25] O. Bajdechi, G. Gielen, J. Huijsing. Optimal Design of Delta-Sigma ADCs by Design Space Exploration. ACM/IEEE DAC 2002.

[26] S. Ganesan, R. Vemuri. Behavioral Partitioning in the Synthesis of Mixed Analog-Digital Systems, ACM/IEEE DAC 2001.

[27] J. Dawson, S. Boyd, M. del Mar Hershenson, T. Lee. Optimal Allocation of Local Feedback in Multistage Amplifiers via Geometric Programming. IEEE Trans. CAS-I 2001.

[28] S.K. Tiwary, S.Velu, R.A. Rutenbar and T. Mukherjee: Pareto-Optimal Modeling for Efficient PLL Optimization. NSTI Nanotech 2004, Boston

[29] K. Antreich, H. Graeb, C. Wieser: Circuit analysis and optimization driven by worst-case distances. IEEE Trans. CAD 1994.

[30] www.muneda.com 


\title{
Mixed Signal Design Space Exploration through Analog Platforms
}

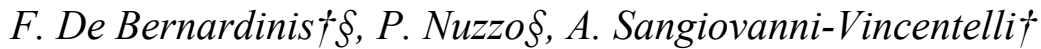

Department of Electrical Engineering, University of California, Berkeley

$\S$ Dipartimento di Ingegneria and Computer Science dell'Informazione, Università di Pisa, Italy

\begin{abstract}
We propose a hierarchical mixed signal design methodology based on the principles of PlatformBased Design (PBD). The methodology is a meet-in-the-middle approach where design components are modeled bottom-up at various abstraction levels and performance constraints are mapped top-down to select among the available components the ones that best meet the constraints. The design methodology can seamlessly operate on both analog and digital designs, thus dealing with mixed signal designs in a consistent way. We demonstrate the effectiveness of the approach optimizing an $80 \mathrm{MS} / \mathrm{s} 14$ bit pipelined Analog-to-Digital Converter (ADC) including digital calibration, yielding $64 \%$ power reduction compared to the original hand optimized design.
\end{abstract}

General Terms: Algorithms

\section{REFERENCES}

[1] H. Liu, A. Singhee, R. Rutenbar, and L. R. Carley, "Remembrance of circuits past: Macromodeling by data mining in large analog design spaces," in Proceedings of DAC, 2002.

[2] T. Kiely and G. Gielen, "Performance modeling of analog integrated circuits using least-squares support vector machines," in Proc. of DATE, 2004.

[3] G. Stehr, H. Graeb, and K. Antreich, "Analog performance space exploration by fourier-motzkion elimination with application to hierarchical sizing," in Proc. of ICCAD, 2004.

[4] A. Sangiovanni Vincentelli, L. Carloni, F. De Bernardinis, and M. Sgroi, "Benefits and challenges for platformbased design," in Proceedings of DAC, pp. 409-414, June 2004.

[5] F. D. Bernardinis, M. I. Jordan, and A. S. Vincentelli, "Support Vector Machines for Analog Circuit Performance Representation," in Proceedings of DAC, June 2003.

[6] J. Platt, "Sequential minimal optimization: A fast algorithm for training support vector machines," Tech. Rep. MSR-TR-98-14, Microsoft Research, 1998.

[7] P. Bunus and P. Fritzson, "A debugging scheme for declarative equation based modeling languages," in Practical Aspects of Decl. Languages : 4th Int. Symp., p. 280, 2002.

[8] B. Murmann and B. E. Boser, "A 12-bit 75-MS/s, Pipelined ADC Using Open-Loop Residue Amplification," IEEE JSSC, vol. 38, pp. 2040-2050, December 2003. 


\title{
Performance Space Modeling for Hierarchical Synthesis of Analog Integrated Circuits
}

\author{
Georges Gielen, Trent McConaghy, Tom Eeckelaert \\ Katholieke Universiteit Leuven, ESAT-MICAS, Leuven, Belgium
}

\begin{abstract}
Automated analog sizing is becoming an unavoidable solution for increasing analog design productivity. The complexity of typical analog SoC subsystems however calls for efficient methods that can handle design hierarchy, in terms of both performance estimation and hierarchical design optimization method. This paper discusses and compares recent developments in this area, with special emphasis on automated modeling and on multi-objective bottom-up hierarchical design.
\end{abstract}

Keywords: Hierarchical Synthesis

\section{REFERENCES}

[1] A. Agarwal, H. Sampath, V. Yelamanchili, and R. Vemuri. Fast and accurate parasitic capacitance models for layout-aware. In Proceedings Design Automation Conference, pages 145-150, 2004.

[2] G. Alpaydin, S. Balkir, and G. Dundar. An Evolutionary Approach to Automatic Synthesis of High-Performance Analog Integrated Circuits. IEEE Trans. on Evol. Computation, 7(3):240-252, 2003.

[3] K. Antreich, J. Eckmueller, H. Graeb, M. Pronath, F. Schenkel, R. Schwencker, and S. Zizala. WiCkeD: Analog Circuit Synthesis Incorporating Mismatch. In Proceedings Custom Integrated Circuits Conference, 2000.

[4] Cadence. NeoCircuit Product. Inc http://www.cadence.com, 2005.

[5] Cadence. UltraSim Product. http://www.cadence.com, 2005.

[6] H. Chang, E. Charbon, U. Choudhury, A. Demir, E. Felt, E. Liu, A. Malvasi, A. L. Sangiovanni-Vincentelli, and I. Vassiliou. A Top-Down, Constraint-Driven Design Methodology for Analog Integrated Circuits. Kluwer Academic Publishers, 1997.

[7] J. M. Cohn, D. J. Garrod, R. A. Rutenbar, and L. R. Carley, editors. Analog Device-Level Layout Automation. Kluwer Academic Publishers, 1994.

[8] W. Daems, G. Gielen, and W. Sansen. Simulation-Based Generation of Posynomial Performance Models for the Sizing of Analog Integrated Circuits. IEEE Transactions on Computer-Aided Design of Integrated Circuits and Systems, 22(5):517-534, 2003.

[9] F. De Bernardinis, M. I. Jordan, and A. L. Sangiovanni-Vincentelli. Support Vector Machines for Analog Circuit Performance Representation. In Proceedings Design Automation Conference, pages 964-969, 2003.

[10] B. De Smedt and G. G. E. Gielen. Holmes: Capturing the Yield-Optimized Design Space Boundaries of Analog and RF Integrated Circuits. In Proceedings Design Automation and Test in Europe Conference, page 10256, 2003.

[11] B. De Smedt and G. G. E. Gielen. WATSON: Design Space Boundary Exploration and Model Generation for Analog and RF IC Design. IEEE Transactions on Computer-Aided Design of Integrated Circuits and Systems, 22(2):213-224, Feb. 2003.

[12] A. Doboli, N. Dhanwada, A. Nunez-Aldana, and R. Vemuri. A Two-Layer Library-Based Approach to Synthesis of Analog Systems from VHDL-AMS Specifications. ACM Transactions on Design Automation of Electronic System, 9(2):238-271, Apr. 2004.

[13] N. Dong and J. Roychowdhury. Piecewise Polynomial Nonlinear Model Order Reduction. In Proceedings Design Automation Conference, pages 484-489, 2003.

[14] S. Donnay, G. G. E. Gielen, W. Sansen, W. Kruiskamp, D. Leenaerts, S. Buytaert, K. Marent, M. Buckens, and C. Das. Using Top-Down CAD Tools for Mixed Analog/Digital ASIC's: a Practical Design Case. Journal of Analog Integrated Circuits and Signal Processing, 10:101-117, 1996.

[15] T. Eeckelaert, T. McConaghy, and G. G. E. Gielen. Efficient Multiobjective Synthesis of Analog Circuits using Hierarchical Pareto-optimal Performance Hypersurfaces. In Proceedings Design Automation and Test in Europe Conference, pages 1070-1075, 2005.

[16] G. Gielen and W. Sansen. Symbolic Anaysis for Automated Design of Analog Integrated Circuits. Kluwer, 1991.

[17] G. E. Gielen. Computer-Aided Design of Analog and Mixed-Signal Integrated Circuits. In R. Rutenbar, G. Gielen, , and B. Antao, editors, Computer Aided Design of Analog Integrated Circuits and Systems, chapter 1, pages 3-30. IEEE Press, Piscataway, NJ, 2002. 
[18] R. Harjani and J. Shao. Feasibility and Performance Region Modeling of Analog an Digital Circuits. Journal of Analog Integrated Circuits and Signal Processing, 10(1):23-43, June 1996.

[19] M. Hershenson. Design of Pipeline Analog-To-Digital Converters Via Geometric Programming. In Proceedings IEEE/ACM International Conference on Computer Aided Design, pages 317-324, 2002.

[20] M. Hershenson, S. Boyd, and T. Lee. GPCAD: A Tool for CMOS Op-Amp Synthesis. In Proceedings IEEE/ACM International Conference on Computer Aided Design, pages 296-303, 1998.

[21] T. Kiely and G. G. E. Gielen. Performance Modeling of Analog Integrated Circuits Using Least-Squares Support Vector Machines. In Proceedings Design Automation and Test in Europe Conference, pages 448-453, 2004.

[22] M. Krasnicki, R. Phelps, R. A. Rutenbar, and L. R. Carley. MAELSTROM: Efficient Simulation-Based Synthesis for Custom Analog Cells. In Proceedings Design Automation Conference, pages 945-950, 1990.

[23] H. Liu, A. Singhee, R. Rutenbar, and L. Carley. Remembrance of Circuits Past: Macromodeling by Data Mining in Large Analog Design Spaces. In Proceedings Design Automation Conference, pages 437-442, 2002.

[24] T. McConaghy, T. Eeckelaert, and G. G. E. Gielen. CAFFEINE: Template-Free Symbolic Model Generation of Analog Circuits via Canonical Form Functions and Genetic Programming. In Proceedings Design Automation and Test in Europe Conference, Mar 2005.

[25] T. McConaghy and G. Gielen. Analysis of Simulation-Driven Numerical Performance Modeling Techniques for Application to Analog Circuit Optimization. In ISCAS, May 2005.

[26] MentorGraphics. Eldo Mach Product. http://www.mentor.com, 2005.

[27] Muneda. WiCkeD Product. http://www.muneda.com, 2005.

[28] Nassda. Hsim Product. http://www.nassda.com, 2005.

[29] Orora. Arsyn Product. http://www.orora.com, 2005.

[30] R. Phelps, M. Krasnicki, R. A. Rutenbar, L. R. Carley, and J. R. Hellums. A Case Study of Synthesis for Industrial-Scale Analog IP: Redesign of the Equalizer/Filter Frontend for an ADSL CODEC. In Proceedings Design Automation Conference, pages 1-6, 2000.

[31] J. Phillips. A Statistical Perspective on Nonlinear Model Order Reduction. In Behavioral Modeling and Simulation Workshop, 2003.

[32] J. Phillips:2003a, J. Afonso, A. Oliveira, and L. Silveira. Analog Macromodeling Using Kernel Methods. In Proceedings IEEE/ACM International Conference on Computer Aided Design, pages 446-443, 2003.

[33] L. Pillage and R. Rohrer. Asymptotic Waveform Evaluation. IEEE Transactions on Computer-Aided Design of Integrated Circuits and Systems, pages 352-366, April 1990.

[34] M. Rewienski and J. White. A Trajectory Piecewise-Linear Approach to Model-Order Reduction and Fast Simulation of Nonlinear Circuits and Micromachined Devices. In Proceedings IEEE/ACM International Conference on Computer Aided Design, pages 252-257, 2001.

[35] D. Root, J. Wood, and N. Tufillaro. New Techniques for Non-linear Behavioral Modeling of Microwave/RF ICs from Simulation and Nonlinear Microwave Methods. In Proc. the ACM International Symposium on Physical Design, pages 76-83, 2000.

[36] J. Roychowdhury. Automated Macromodel Generation for Electronic Systems. In Behavioral Modeling and Simulation Workshop, 2003.

[37] F. Schenkel, M. Pronath, S. Zizala, R. Schwencker, H. Graeb, and K. Antreich. Mismatch Analysis and Direct Yield Optimization by Spec-Wise Linearization and Feasibility-Guided Search. In Proceedings Design Automation Conference, 2001.

[38] G. Stehr, H. Graeb, and K. Antreich. Feasibility Regions and their Significance to the Hierarchical Optimization of Analog and Mixed-Signal Systems. International Series of Numerical Mathematics, 146:167-184, 2003.

[39] G. Stehr, H. Graeb, and K. Antreich. Performance Trade-off Analysis of Analog Circuit By Normal-Boundary Intersection. In Proceedings Design Automation Conference, pages 958-963, 2003.

[40] Synopsys. Circuit Explorer Product. http://www.synopsys.com, 2005.

[41] Synopsys. Nanosim Product. http://www.synopsys.com, 2005.

[42] G. Van der Plas, G. Debyser, F. Leyn, K. Lampaert, J. Vandenbussche, G. G. E. Gielen, W. Sansen, P. Veselinovic, and D. Leenaerts. AMGIE - A Synthesis Environment for CMOS Analog Integrated Circuits. IEEE Transactions on Computer-Aided Design of Integrated Circuits and Systems, 20(9):1037-1058, Sept. 2001.

[43] D. A. Van Veldhuizen. Multiobjective Evolutionary Algorithms: classifications, analysis, and new innovations. $\mathrm{PhD}$ thesis, Air Force Institute of Technology, Wright-Patterson AFB, USA, June 1999.

[44] P. Vancorenland, G. V. der Plas, M. Steyaert, G. Gielen, and W. Sansen. A Layout-aware Synthesis Methodology for RF Circuits. In Proceedings IEEE/ACM International Conference on Computer Aided Design, pages 358-, 2001. 
[45] G. Wolfe, M. Ding, and R. Vemuri. Adaptive Sampling and Modeling of Analog Circuit Performance Parameters. In Proc. VLSI-SOC, pages 142-, 2003.

[46] G. Wolfe and R. Vemuri. Extraction and Use of Neural Network Models in Automated Synthesis of Operational Amplifiers. IEEE Transactions on Computer-Aided Design of Integrated Circuits and Systems, 22(2), 2003.

[47] G. Zhang, E. A. Dengi, R. A. Rohrer, R. A. Rutenbar, and L. R. Carley. A Synthesis Flow Toward Fast Parasitic Closure for Radio-Frequency Integrated Circuits. In Proceedings Design Automation Conference, pages 155-158, 2004.

[48] E. Zitzler, K. Deb, and L. Thiele. Comparison of Multiobjective Evolutionary Algorithms: Empirical Results. Evolutionary Computation, 8(2):173-195, 2000. 
Panel Moderator: Ron Wilson- Electronic Engineering Times

Panelists:

Chris Hamlin - LSI Logic Corp., Milpitas, CA

Ken McElvain - Synplicity Inc., Sunnyvale, CA

Steve Leibson - Tensilica Inc., Santa Clara, CA

Ivo Bolson - Xilinx Inc., San Jose, CA

Rich Tobias - Toshiba America Inc., New York, NY

Raul Camposano - Synopsys Inc., Mountain View, CA

\begin{abstract}
Moore's law delivers higher performance and lower cost for FPGAs and ASICs alike, but at the 90nm process node and below, design schedules using the traditional cell-based ASIC design methodology hit a wall of uncertainty. At 90nm and below an emerging alternative ASIC design platform is either Platform ASIC or FPGAs. Which way will the cell-based ASIC designer turn for their next design?

Over time, FPGAs and structured/platform ASICs are together poised to replace today's cellbased ASIC market, but which is the real answer to future digital design? Can companies really use these platforms to achieve the system cost reduction and functionality that they need to stay competitive? Which applications will migrate to these platforms the fastest? Is it possible to just tweak the existing cell-based methodology to more efficiently reach the benefits of 90nm process nodes and below? This lively panel will discuss whether it is FPGAs, structured/platform ASICs, or something else that stand to gain the most ground from the projected \$25B ASIC market, and why.

Keywords: Digital Design and Programmable ASIC Platforms
\end{abstract}



Real-Time Systems with Energy Constraints

\author{
Luis Alejandro Cortés ${ }^{1,2}$, Petru Eles ${ }^{2}$, Zebo Peng ${ }^{2}$ \\ ${ }^{1}$ Volvo Truck Corporation, Gothenburg, Sweden \\ ${ }^{2}$ Linköping University, Linköping, Sweden
}

\begin{abstract}
There exist real-time systems for which it is possible to trade off precision for timeliness. In these cases, a function assigns reward to the application depending on the amount of computation allotted to it. At the same time, many such applications run on battery-powered devices with stringent energy constraints. This paper addresses the problem of maximizing rewards subject to time and energy constraints. We propose a quasi-static approach where the problem is solved in two steps: first, at design-time, a number of solutions are computed and stored (off-line phase); second, one of the precomputed solutions is selected at run-time based on actual values of time and energy (on-line phase). Thus our approach is able to exploit, with low on-line overhead, the dynamic slack caused by tasks executing less number of cycles than in the worst case. We conduct numerous experiments in order to show the advantages of our approach.
\end{abstract}

Keywords: Quasi-Static, Dynamic Voltage Scaling

\title{
REFERENCES
}

[1] L. A. Cortés, P. Eles, and Z. Peng. Quasi-Static Scheduling for Real-Time Systems with Hard and Soft Tasks. In Proc. DATE Conference, pp. 1176-1181, 2004.

[2] L. A. Cortées. Verification and Scheduling Techniques for Real-Time Embedded Systems. PhD thesis, Department of Computer and Information Science, Linköping University, Mar. 2005.

[3] D. L. Hull. An Environment for Imprecise Computations. PhD thesis, Department of Computer Science, University of Illinois, Urbana-Champaign, Jan. 2000.

[4] J. W. Liu, W.-K. Shih, K.-J. Lin, R. Bettati, and J.-Y. Chung. Imprecise Computations. Proc. IEEE, 82(1):83-94, Jan. 1994.

[5] S. M. Martin, K. Flautner, T. Mudge, and D. Blaauw. Combined Dynamic Voltage Scaling and Adaptive Body Biasing for Low Power Microprocessors under Dynamic Workloads. In Proc. ICCAD, pp. 721-725, 2002.

[6] NEC Memories. http://www.necel.com/memory/index_e.html.

[7] Y. Nesterov and A. Nemirovski. Interior-Point Polynomial Algorithms in Convex Programming. SIAM, Philadelphia, 1994.

[8] T. Okuma, H. Yasuura, and T. Ishihara. Software Energy Reduction Techniques for Variable-Voltage Processors. IEEE Design \& Test of Computers, 18(2):31-41, Mar. 2001.

[9] C. Rusu, R. Melhem, and D. Mossé. Maximizing Rewards for Real-Time Applications with Energy Constraints. ACM Trans. on Embedded Computing Systems, 2(4):537-559, Nov. 2003. 


\title{
DC-DC Converter-Aware Power Management for Battery-Operated Embedded Systems
}

\author{
Yongseok Choi*, Naehyuck Chang*, Taewhan Kim** \\ *School of Computer Science \& Engineering, Seoul National University, Seoul, Korea \\ **School of Electrical Engineering, Seoul National University, Seoul, Korea
}

\begin{abstract}
Most digital systems are equipped with DC-DC converters to supply various levels of voltages from batteries to logic devices. DC-DC converters maintain legal voltage ranges regardless of the load current variation as well as battery voltage drop. Although the efficiency of DC-DC converters is changed by the output voltage level and the load current, most existing power management techniques simply ignore the efficiency variation of DC-DC converters. However, without a careful consideration of the efficiency variation of DC-DC converters, finding a true optimal power management will be impossible. In this work, we solve the problem of energy minimization with the consideration of the characteristics of power consumption of DC-DC converter. Specifically, the contributions of our work are: (1) We analyze the effects of the efficiency variation of DC-DC converters on a single task execution in DVS (dynamic voltage scaling) scheme, and propose a technique, called DC DVS, of DC-DC converter-aware energyminimal DVS; (2) DC DVS is then extended to combine the effects of DC-DC converters with the procedures of general DVS techniques with multiple tasks; (3) Conversely, we propose a technique, called DC CONF, of generating a DC-DC converter that is best suited, in terms of total energy efficiency, to the intended application, and (4) finally, we complete our integrated framework DC-lp, which is based on DC DVS and DC CONF, that attempts to solve the DC-DC converter configuration selection problem and the DVS problem simultaneously. To show the effectiveness of the proposed techniques, a set of experimental results is provided. In summary, it is shown that DC-1p is able to save $16.0 \% » 22.1 \%$ of energy on the average, which otherwise was dissipated in the previous power management schemes with no consideration of DC-DC converter efficiency variation.
\end{abstract}

Keywords: Low power, DC-DC converter, Voltage scaling

\section{REFERENCES}

[1] V. Kursun, S. G. Narendra, V. K. De and E. G. Friedman, "Monolithic DC-DC converter analysis and MOSFET gate voltage optimization," ISQED, 2003.

[2] M. M. Jovanovic, M. T. Zhang and F. C. Lee, "Evaluation of synchronous-rectification efficiency improvement limits in forward converters," IEEE Transactions on Industrial Electronics, August 1995.

[3] T. Simunic, L. Benini and G. De Micheli, "Cycle-accurate simulation of energy consumption in embedded systems," $D A C, 1999$.

[4] M. Pedram and Q. Wu, "Design considerations for battery-powered electronics," $D A C, 1999$.

[5] F. Yao, A. Demers and A. Shenker, "A scheduling model for reduced CPU energy," IEEE Foundations of Computer Science, 1995.

[6] G. Quan and X. S. Hu, "Minimum energy fixed-priority scheduling for variable voltage processors," DATE, 2002.

[7] W. Kim, D. Shin, H. S. Yun, J. Kim and S. L. Min, "Performance comparison of dynamic voltage scaling algorithms for hard real-time systems," RTAS, 2002.

[8] D. Shin, J. Kim and S. Lee, "Intra-task voltage scheduling for low-energy hard real-time applications," IEEE Design and Test of Computers, March 2001.

[9] W. Kwon and T. Kim, "Optimal voltage allocation techniques for dynamically variable voltage processors," $D A C, 2003$.

[10] L. H. Chandrasena, P. Chandrasena, and M. J. Liebelt, "An Energy Efficient Rate Selection Algorithm for Voltage Quantized Dynamic Voltage Scaling," ISSS, 2001.

[11] C. Locke, D. Vogel, and T. Mesler, "Building a Predictable Avionics Platform in Ada: A Case Study," RTSS, 1991. 
[12] N. Kim, M. Ryu, S. Hong, M. Saksena, C. Choi, and H. Shin "Visual Assessment of a Real-time System Design: A Case Study on a CNC Controller," RTSS, 1996.

[13] Micrel Semiconductor, "MIC4685 Datasheet," "http://www.micrel.com". 


\title{
Energy Optimal Speed Control of Devices with Discrete Speed Sets
}

\author{
Ravishankar Rao and Sarma Vrudhula \\ NSF Center for Low Power Electronics, Computer Science and Engineering Department, \\ Arizona State University, Tempe, AZ
}

\begin{abstract}
We obtain analytically, the energy optimal speed profile of a generic multi-speed device with a discrete set of speeds, to execute a given task within a given time. Current implementations of energy efficient speed control policies (including DVFS) almost exclusively use the minimum feasible speed pair, which has been shown before to be suboptimal. Unlike previous works, ours does not require an explicit functional relationship between the device's power and speed (e.g. the CMOS power model), but only assumes that the power-speed relationship is a W-convex (a discrete equivalent of a convex) function. This assumption allowed us to show that the optimal speed profile uses at most two speeds, and that all the essential characteristics of the power-speed relationship can be encapsulated within a single speed, wu. The latter speed is intrinsic to the device (i.e. task independent) and can be readily computed from its power-speed values (without any curve fit). Further, wu is also the speed at which the the device consumes the least energy per unit work done. The problem formulation reduces to a linear program in the number of supported speeds, which in general, is difficult to solve analytically. However, the optimum solution has a very simple form - it is either wu, or the minimum feasible speed pair for the given task. We verified that a number of commercial DVFS processors, and other devices like
\end{abstract} disk drives satisfied our model of the $\mathrm{W}$-convex power-speed relationship.

Keywords: voltage scaling, frequency scaling, speed control, low-power, convex functions, energy optimization

\section{REFERENCES}

[1] Advanced Micro Devices. AMD Athlon 64 Processor Power and Thermal Data Sheet.

[2] M. S. Bazaara, H. D. Sherali, and C. M. Shetty. Nonlinear Programming: Theory and Algorithms. John Wiley and Sons, second edition, 1993.

[3] L. Benini, A. Bogliolo, G. A. Paleologo, and G. De Micheli. Policy optimization for dynamic power management. IEEE Trans. CAD, 18(6):813-833, June 1999.

[4] N. Chang, I. Choi, and H. Shim. DLS: Dynamic backlight luminance scaling of liquid crystal display. IEEE Trans. VLSI Sys., 12(8):837-846, August 2004.

[5] M. Fleischmann. Longrun power managementTM: Dynamic power management for Crusoe ${ }^{\mathrm{TM}}$ processors.

[6] S. Gurumurthi, A. Sivasubramaniam, M. Kandemir, and H. Franke. Reducing disk power consumption in servers with DRPM. IEEE Computer, 36(12):59-66, December 2003.

[7] Intel Corp. Enhanced Intel SpeedStep Technology for the Intel Pentium M Processor.

[8] Intel Corp. Intel Pentium M Processor on 90nm Process with 2-MB L2 Cache.

[9] Intel Corp. Intel PXA26x Processor Family : Electrical, Mechanical, and Thermal Specification.

[10] S. Irani, S. Shukla, and R. Gupta. Algorithms for power savings. In Proc. ACM-SIAM Symposium on Discrete Algorithms, pages 37-46, Philadelphia, PA, USA, 2003. Society for Industrial and Applied Mathematics.

[11] S. Irani, S. Shukla, and R. Gupta. Online strategies for dynamic power management in systems with multiple power-saving states. ACM Trans. Embedded Computing Sys. (TECS), 2(3):325-346, 2003.

[12] T. Ishihara and H. Yasuura. Voltage scheduling problem for dynamically variable voltage processors. In Proc. Intl' Symp. Low Power Electronics and Design (ISLPED), pages 197-202, 1998.

[13] R. Jejurikar, C. Pereira, and R. Gupta. Leakage aware dynamic voltage scaling for real-time embedded systems. In Proc. Design Automation Conf. (DAC), pages 275-280, 2004.

[14] J. R. Lorch and A. J. Smith. PACE: A new approach to dynamic voltage scaling. IEEE Trans. Computers, 53(7):856-869, July 2004.

[15] S. M. Martin, K. Flautner, T. Mudge, and D. Blaauw. Combined dynamic voltage scaling and adaptive body biasing for lower power microprocessors under dynamic workloads. In Proc. ICCAD, pages 721-725, 2002. 
[16] A. Miyoshi, C. Lefurgy, E. V. Hensbergen, R. Rajamony, and R. Rajkumar. Critical power slope: Understanding the runtime effects of frequency scaling. In Proc. Intl' Conf. Supercomputing (ICS), pages 35-44, 2002.

[17] K. Okada, N. Kojima, and K. Yamashita. A novel drive architecture of HDD: "multimode hard disc drive". In Proc. Intl' Conf. Consumer Electroncis (ICCE), pages 92-93. IEEE Press, 2000.

[18] J. Pouwelse, K. Langendoen, and H. Sips. Application-directed voltage scaling. IEEE Trans. VLSI Sys., 11(5):812-826, October 2003.

[19] Q. Qiu, Q. Wu, and M. Pedram. Stochastic modeling of a power-managed system-construction and optimization. IEEE Trans. CAD, 20(10):1200-1217, October 2001.

[20] R. Rao. Energy optimal speed control for components of portable systems. Master's thesis, University of Arizona, Tucson, 2004.

[21] R. Rao and S. Vrudhula. Energy optimization for a two-device data flow chain. In Proc. Intl' Conf. ComputerAided Design (ICCAD), pages 268-274, November 2004.

[22] R. Rao, S. Vrudhula, and M. S. Krishnan. Disk drive energy optimization for audio-video applications. In Proc. Conf. Compilers, Arch., Synth. Emb. Sys. (CASES), pages 93-103, September 2004.

[23] C. Schurgers, O. Aberthorne, and M. Srivastava. Modulation scaling for energy aware communication systems. In Proc. Intl' Symp. Low Power Electronics and Design (ISLPED), pages 96-99. ACM Press, 2001.

[24] M. Weiser, B. Welch, A. Demers, and S. Shenker. Scheduling for reduced CPU energy. In Proc. Symp. Operating Sys. Design and Implementation (OSDI), pages 13-23, 1994. 


\title{
Optimal Procrastinating Voltage Scheduling for Hard Real-Time Systems
}

Yan Zhang, Zhijian Lu, John Lach, Kevin Skadron, Mircea R. Stan

University of Virginia, Charlottesville, VA, U.S.A.

\begin{abstract}
This paper presents an optimal procrastinating voltage scheduling (OP-DVS) for hard real-time systems using stochastic workload information. Algorithms are presented for both single-task and multi-task workloads. Offline calculations provide real-time guarantees for worst-case execution, and online scheduling reclaims slack time and schedules tasks accordingly. The OPDVS algorithm is provably optimal in terms of energy minimization with no deadline misses. Simulation results show up to $30 \%$ energy savings for single-task workloads and $74 \%$ for multitask workloads compared to using a constant worst-case execution voltage. The complexity of the algorithm for multi-task workloads is linear to the number of tasks involved.

Keywords: Power Management, Dynamic Voltage Scaling, Real-time Scheduling, Optimization Algorithm

\section{REFERENCES}

[1] D. Grunwald, et al. Policies for Dynamic Clock Scheduling. In Proc. of the 4th Symposium on OSDI, 2000.

[2] Y-H. Lee and C. Krishna. Voltage-Clock Scaling for Low Power Energy Consumption in Real-Time Embedded Systems. In Proc. of the 6th Intl. Conference RTCSA, 1999.

[3] T. Pering, T. Burd, and R. Brodersen. Voltage Scheduling in the lpARM Microprocessor System. In Proc. of ISLPED, 2000.

[4] J. R. Lorch and A.J. Smith. PACE: A New Approach to Dynamic Voltage Scaling. IEEE Tran. on Computers, July 2004.

[5] F. Gruian. Hard Real-Time Scheduling for Low-Energy Using Stochastic Data and DVS Processors. In Proc. of ISLPED, 2001.

[6] W. Yuan and K. Nahrstedt. Energy-Efficient Soft Real-Time CPU Scheduling for Mobile Multimedia Systems. In Proc. of SOSP, 2003.

[7] D. Roychowdhury, I. Koren, C. M. Krishna, Y.H.Lee. A voltage scheduling heuristic for real-time task graphs. In Proc. of Intl. Conf. On Dependable Systems and Networks, June 2003.

[8] F. Gruian and K. Krzysztof. Uncertainty-Based Scheduling: Energy-Efficient Ordering for Tasks with Variable Execution Time. In Proc. of ISLPED, 2003.

[9] C. Rusu, et al. Maximizing the System Value while Satisfying Time and Energy Constraints. In Proc. RTSS'02, 2002.

[10] L. Leung, C. Tsui, and W. Ki, Minimizing Energy Consumption of Hard Real-Time Systems with Simultaneous Tasks Scheduling and Voltage Assignment using Statistical Data, ASP-DAC, 2004.
\end{abstract}




\title{
Flexible ASIC: Shared Masking for Multiple Media Processors
}

\author{
Jennifer L. Wong*, Farinaz Kourshanfar**, Miodrag Potkonjak* \\ *Univ. of Calif., Los Angeles, Los Angeles, California \\ **Univ. of Calif., Berkeley, Berkeley, California
}

\begin{abstract}
ASIC provides more than an order of magnitude advantage in terms of density, speed, and power requirement per gate. However, economic (cost of masks) and technological (deep micron manufacturability) trends favor FPGA as an implementation platform. In order to combine the advantages of both platforms and alleviate their disadvantages, recently a number of approaches, such as structured ASIC/regular fabrics, have been proposed. Our goal is to introduce an approach that has the same objective, but is orthogonal to those already proposed. The idea is to implement several ASIC designs in such a way that they share the datapath, memory structure, and several bottom layers of interconnect, while each design has only a few unique metal layers. We identified and addressed two main problems in our quest to develop a CAD flow for realization of such designs. They are: (i) the creation of the datapath, and (ii) the identification of common and unique interconnects for each design. Both problems are solved optimally using ILP formulations. We assembled a design flow platform using two new programs and the Trimaran and Shade tools. We quantitatively analyzed the advantages and disadvantages of the approach using the Mediabench benchmark suite.
\end{abstract}

Keywords: ASIC, interconnect, optimization

\section{REFERENCES}

[1] R. F. Cmelik and D. Keppel. Shade: A fast instruction-set simulator for execution profiling. Technical Report SMLI 93-12, UWCSE 93-06-06, 1993.

[2] J. Cong, Y. Fan, X. Yang, and Z. Zhang. Architecture and synthesis for multi-cycle communication. In International Symposium on Physical Design, pages 190-196, 2003.

[3] M. Garey and D. Johnson. Computers and Intractability. W.H. Freeman, 1979.

[4] http://www.fma.fujitsu.com/accel/main01.asp.

[5] http://www.lsilogic.com/products/rapidchip_platform_asic/index.html.

[6] B. Hu, H. Jiang, Q. Liu, and M. Marek-Sadowska. Synthesis and placement flow for gain-based programmable regular fabrics. In International Symposium on Physical Design, pages 197-203, 2003.

[7] A. Kahng, I. Bolsens, J. Cohn, B. Gupta, C. Hamlin, Z. Orbach, and L. Pileggi. What is the next implementation fabric? IEEE Design and Test of computers, 20(6):86-95, Nov. 2003.

[8] V. Kheterpal, A. J. Strojwas, and L. Pileggi. Routing architecture exploration for regular fabrics. In Conference on Design Automation, pages 204-207, 2004.

[9] D. E. Lackey, P. S. Zuchowski, and J. Koehl. Designing mega-ASICs in nanogate technologies. In Conference on Design Automation, pages 770-775, 2003.

[10] C. Lee, W. Mangione-Smith, and M. Potkonjak. Mediabench: A tool for evaluating multimedia and communication systems. In MICRO-30 Conference, pages 330-335, Nov. 1997.

[11] F. Mo and R. K. Brayton. Fishbone: a block-level placement and routing scheme. In International Symposium on Physical Design, pages 204-209, 2003.

[12] T. Okamoto, T. Kimoto, and N. Maeda. Design methodology and tools for NEC electronics' structured ASIC ISSP. In International Symposium on Physical Design, pages 90-96, 2004.

[13] L. Pileggi, et al. Exploring regular fabrics to optimize the performance-cost trade-off. In Conference on Design Automation, pages 782-787, 2003.

[14] Y. Ran and M. Marek-Sadowska. On designing via-configurable cell blocks for regular fabrics. In Conference on Design Automation, pages 198-203, 2004.

[15] D. D. Sherlekar. Design considerations for regular fabrics. In International Symposium on Physical Design, pages $97-102,2004$.

[16] Trimaran. http://www.trimaran.org/. 
[17] K. Wu and Y. Tsai. Structured ASIC, evolution or revolution? In International Symposium on Physical Design, pages 103-106, 2004.

[18] P. S. Zuchowski, C. B. Reynolds, R. J. Grupp, S. G. Davis, B. Cremen, and B. Troxel. A hybrid ASIC and FPGA architecture. In International Conference on Computer-aided Design, pages 187-194, 2002. 


\title{
Device And Architecture Co-Optimization for FPGA Power Reduction
}

Lerong Cheng, Phoebe Wong, Fei Li, Yan Lin, and Lei He

Electrical Engineering Department, University of California, Los Angeles, CA

\begin{abstract}
Device optimization considering supply voltage Vdd and threshold voltage Vt tuning does not increase chip area but has a great impact on power and performance in the nanometer technology. This paper studies the simultaneous evaluation of device and architecture optimization for FPGA. We first develop an efficient yet accurate timing and power evaluation method, called trace-based model. By collecting trace information from cycle-accurate simulation of placed and routed FPGA benchmark circuits and re-using the trace for different $\mathrm{Vdd}$ and $\mathrm{Vt}$, we enable the device and architecture co-optimization for hundreds of combinations. Compared to the baseline FPGA which has the architecture same as the commercial FPGA used by Xilinx, and has Vdd suggested by ITRS but Vt optimized by our device optimization, architecture and device co-optimization can reduce energy-delay product by $20.5 \%$ without any chip area increase compared to the conventional FPGA architecture. Furthermore, considering power-gating of unused logic blocks and interconnect switches, our cooptimization method reduces energy-delay product by $54.7 \%$ and chip area by $8.3 \%$. To the best of our knowledge, this is the first in-depth study on architecture and device co-optimization for FPGAs.
\end{abstract}

Keywords: FPGA, low power, powergating, Ptrace, Psim

\section{REFERENCES}

[1] J. Rose, R. J. Francis, D. Lewis, and P. Chow, "Architecture of field-programmable gate arrays: The effect of logic functionality on area efficiency," Proc. IEEE Int. Solid-State Circuits Conf., 1990.

[2] J. Kouloheris and A. E. Gamal, "FPGA area vs. cell granularity - lookup tables and PLA cells," in $I^{s t} A C M$ Workshop on FPGAs, berkeley, CA, Feb 1992.

[3] E. Ahmed and J. Rose, "The effect of LUT and cluster size on deep-submicron FPGA performance and density," in Proc. ACM Intl. Symp. Field-Programmable Gate Arrays, pp. 3-12, Feb 2000.

[4] F. Li, D. Chen, L. He, and J. Cong, "Architecture evaluation for power-efficient FPGAs," in Proc. ACM Intl. Symp. Field-Programmable Gate Arrays, Feb 2003.

[5] K. Poon, A. Yan, and S. Wilton, "A flexible power model for FPGAs," in Proc. of 12th International conference on Field-Programmable Logic and Applications, Sep 2002.

[6] A. Gayasen, Y. Tsai, N. Vijaykrishnan, M. Kandemir, M. J. Irwin, and T. Tuan, "Reducing leakage energy in FPGAs using region-constrained placement," in Proc. ACM Intl. Symp. Field-Programmable Gate Arrays, February 2004.

[7] F. Li, Y. Lin, L. He, and J. Cong, "Low-power FPGA using pre-defined dual-vdd/dual-vt fabrics," in Proc. ACM Intl. Symp. Field-Programmable Gate Arrays, February 2004.

[8] F. Li, Y. Lin, and L. He, "FPGA power reduction using configurable dual-vdd," in Proc. Design Automation Conf., June 2004.

[9] Fei Li, Yan Lin and Lei He, "Vdd programmability to reduce fpga interconnect power," in Proc. Intl. Conf. Computer-Aided Design, November 2004.

[10] Y. Lin, F. Li and L. He, "Circuits and architectures for vdd programmable FPGAs," in Proc. ACM Intl. Symp. Field-Programmable Gate Arrays, Feb 2005.

[11] International Technology Roadmap for Semiconductor in http://public.itrs.net/, 2002.

[12] L. Cheng, P. Wong, F. Li, Y. Lin, and L. He, "Device and architecture co-optimization for FPGA power reduction," Tech. Rep. 05-258, UCLA Engr. 


\title{
Exploring Technology Alternatives for Nano-Scale FPGA Interconnects
}

\author{
Aman Gayasen, N. Vijaykrishnan, M. J. Irwin \\ Penn State University, University Park
}

\begin{abstract}
Field Programmable Gate Arrays (FPGAs) are becoming increasingly popular. With their regular structures, they are particularly amenable to scaling to smaller technologies. On the other hand, there have been significant advances in nano-electronics fabrication over the past few years. In this paper we explore FPGA devices of the next decade using nano-wires and molecular switches for programmable interconnect, and compare them to traditional SRAM-based FPGAs that use pass transistors as switches (scaled to $22 \mathrm{~nm}$ ). We show that by using nano-wires and molecular switches, it is possible to reduce the area of the FPGA by $70 \%$ and improve performance.
\end{abstract}

Keywords: FPGA, nanotechnology, nanoelectronics, interconnect

\section{REFERENCES}

[1] "Berkeley Predictive Technology Model," http://www-device.eecs.berkeley.edu/ ptm/interconnect.html.

[2] "Floating gate flash demise sees Actel update ProASIC," http://www.electronicsweekly.com, Mar 19, 2003.

[3] International technology roadmap for semiconductors. <http://public.itrs.net/Files/2003ITRS/Home2003.htm> 2003.

[4] Xilinx Product Datasheets, http://www.xilinx.com/literature.

[5] V. Betz and J. Rose, "VPR: A New Packing, Placement and Routing Tool for FPGA Research," In International Workshop on Field-programmable Logic and Applications, 1997.

[6] V. Betz, J. Rose, and A. Marquardt, "Architecture and CAD for Deep-Submicron FPGAs," Kluwer Academic Publishers, February 1999.

[7] Yong Chen et al., "Nanoscale molecular-switch devices fabricated by imprint lithography, " in Applied Physics Letters 82 (2003), no. 10, 1610-1612.

[8] S.C. Goldstein and M. Budiu, "Nanofabrics: Spatial computing using molecular electronics," In Proceedings of the International Symposium on Computer Architecture (ISCA 2001), July 2001.

[9] Andre Dehon and Michael J. Wilson, "Nanowire-Based Sublithographic Programmable Logic Arrays," in Proc. of International Symposium on Field Programmable Gate Arrays, 2004.

[10] Andre Dehon, Patrick Lincoln, John E. Savage, "Stochastic Assembly of Sublithographic Nanoscale Interfaces," in IEEE Transactions on Nanotechnology, Vol. 2, No. 3, Sep 2003.

[11] Jonathan Greene, Esmat Hamdy, and Sam Beal, “Antifuse Field Programmable Gate Arrays,", in Proceedings of the IEEE, Vol. 81, No. 7, Jul 1993.

[12] Jung-Hyurk Lim, Chad A. Mirkin, "Electrostatically Driven Dip-Pen Nanolithography of Conducting Polymers," Adv. Mat., 2002, 14(20), 1474-1477.

[13] Brent A. Mantooth and Paul S. Weiss, "Fabrication, Assembly, and Characterization of Molecular Electronic Components," in Proceedings of the IEEE, Vol 91, No. 11, Nov 2003.

[14] M. C. McAlpine, R. S. Friedman, and C. M. Lieber, "Nanoimprint Lithography for Hybrid Plastic Electronics," Nano Lett. 3, 443-445, 2003.

[15] Arijit Raychowdhury, Kaushik Roy, "A Circuit Model for Carbon Nanotube Interconnects: Comparative Study with $\mathrm{Cu}$ Interconnects for Scaled Technologies," in Proc. of International Conference on Computer Aided Design 2004.

[16] L. Shang, A. S. Kaviani, and K. Bathala, "Dynamic power consumption in Virtex[tm]-II FPGA family,". In Proceedings of ACM/SIGDA International Symposium on Field-programmable gate arrays, 2002.

[17] D. R. Stewart, D. A. A. Ohlberg, P. A. Beck, Y. Chen, R. S. Williams, J. O. Jeppesen, K. A. Nielsen, J. F. Stoddart, "Molecule-Independent Electrical Switching in Pt/Organic Monolayer/Ti Devices," Nano Letters 2004 Vol. 4, No. 1, 133-136.

[18] J. M. Tour, W. L. Van Zandt, C. P. Husband, S. M. Husband, L. S. Wilson, P. D. Franzon, D. P. Nackashi, "Nanocell Logic Gates for Molecular Computing," IEEE Transactions on Nanotechnology 2002, 1, $100-109$.

[19] Yue Wu, Jie Xiang, Chen Yang, Wei Lu, and C. M. Lieber, "Single-crystal metallic nanowires and metal/semiconductor nanowire heterostructures," Nature, Vol. 430, Jul 2004. 


\title{
Piece-wise Approximations of RLCK Circuit Responses using Moment Matching
}

\author{
Chirayu S. Amin*, Yehea I. Ismail*, Florentin Dartu** \\ *ECE, Northwestern University, Evanston, IL \\ **SCL, Intel Corporation, Hillsboro, OR
}

\begin{abstract}
Capturing $R L C K$ circuit responses accurately with existing model order reduction (MOR) techniques is very expensive. Direct metrics for fast analysis of $R C$ circuits exist but there is no such technique for $R L C K$ circuits. This paper introduces a new family of MOR techniques based on piece-wise functions to capture $R L C K$ circuit responses accurately using only four or five moments. The time-domain response is approximated using a piece-wise function whose pieces are simple polynomials. The proposed method is fast and guaranteed stable and it avoids the calculation of poles and residues associated with existing model order reduction techniques. Results for many different industrial netlists indicate that delay and transition time can be captured within 5\% error using only four moments. To the authors' knowledge, there is no existing method that can extract as much information about $R L C K$ circuits with only four or five moments.
\end{abstract}

Keywords: Interconnect timing analysis, moments, $R C, R L C, R L C K$ circuits

\section{REFERENCES}

[1] L. T. Pillage and R. A. Rohrer, "Asymptotic waveform evaluation for timing analysis," TCAD, vol. 9, no. 4, pp. 352-366, April 1990.

[2] P. Feldmann and R. W. Freund, "Efficient linear circuit analysis by Pade approximation via Lanczos process," TCAD, vol. 14, no. 5, pp. 639-649, May 1995.

[3] A. Odabasioglu, M. Celik, L. T. Pileggi, "PRIMA: Passive Reduced-Order Interconnect Macromodeling Algorithm," TCAD, vol. 17, no. 8, pp. 645-654, August 1998.

[4] International Technology Roadmap for Semiconductors (ITRS), 2003 edition, Semiconductor Industry Association (http://public.itrs.net/).

[5] Y. I. Ismail and E. G. Friedman, "Effects of inductance on the propagation delay and repeater insertion in VLSI circuits," TVLSI, vol. 8, no. 2, pp. 195-206, April 2000.

[6] W. C. Elmore, "The transient response of damped linear networks with particular regard to wideband amplifiers," Journal of Applied Physics, v. 19, pp. 55-63, January 1948.

[7] J. L. Wyatt, Circuit Analysis, Simulation and Design, North-Holland, The Netherlands: Elsevier Science, 1987.

[8] T. Lin, E. Acar, and L. Pileggi, "h-gamma: An $R C$ delay metric based on a Gamma distribution approximation to the homogenous response," ICCAD, pp. 19-25, 1998.

[9] F. Liu, C. Kashyap, and C. J. Alpert, "A delay metric for $R C$ circuits based on the Weibull distribution," ICCAD, pp. 620-624, 2002.

[10] C. J. Alpert, F. Liu, C. Kashyap, and A. Devgan, "Delay and slew metrics using the lognormal distribution," $D A C$, pp. 382-385, 2003.

[11] C. J. Alpert, A. Devgan, and C. Kashyap, "A two moment $R C$ delay metric for performance optimization," ISPD, pp. 69-74, 2000.

[12] C. V. Kashyap, C. J. Alpert, and A. Devgan, "An 'effective' capacitance based delay metric for $R C$ interconnect," ICCAD, pp. 229-234, November 2000.

[13] K. Agarwal, D. Sylvester, and D. Blaauw, "Simple metrics for slew rate of $R C$ circuits based on two circuit moments," DAC, pp. 950-953, 2003.

[14] C. Kashyap, C. J. Alpert, A. Devgan, and F. Liu, "PERI: A technique for extending delay and slew metrics for ramp inputs," Proc. of Workshop on Timing Issues in Digital Systems, pp. 57-62, 2002.

[15] B. Tutuianu, F. Dartu, and L. Pileggi, "An explicit Rc-circuit delay approximation based on the first three moments of the impulse response," DAC, pp. 611-616, 1996.

[16] C. L. Ratzlaff, N. Gopal, and L. T. Pillage, "RICE: Rapid Interconnect Circuit Evaluator,” DAC, pp. 555-560, June 1991.

[17] M. Celik, L. Pileggi, A. Odabasioglu, IC Interconnect Analysis, Kluwer Academic Publications, Boston, 2002. 


\author{
Kin Cheong Sou, Alexandre Megretski, Luca Daniel \\ Department of Electrical Engineering and Computer Science, Massachusetts Institute of \\ Technology, Cambridge MA, USA
}

\begin{abstract}
In this paper an optimization based model order reduction (MOR) framework is proposed. The method involves setting up a quasiconvex program that explicitly minimizes a relaxation of the optimal Honorm MOR problem. The method generates guaranteed stable and passive reduced models and it is very flexible in imposing additional constraints. The proposed optimization approach is also extended to parameterized model reduction problem (PMOR). The proposed method is compared to existing moment matching and optimization based MOR methods in several examples. A PMOR model for a large RF inductor is also constructed.
\end{abstract}

Keywords: parameterized model order reduction, quasi-convex optimization, ellipsoid algorithm, RF inductor

\title{
REFERENCES
}

[1] B. Anderson, M. Mansour, and F. Kraus. A New Test For Strict Positive Realness. IEEE Trans on Circuit and Systems I: Fundamental Theory and Applications, 42:226-229, 1995.

[2] D. Bertsimas and J. Tsitsiklis. Introduction to Linear Optimization. Athena Scientific, 1997.

[3] W. Beyene and Schutt-Aine. Efficient Transient Simulation of High-Speed Interconnects Characterized by Sampled Data. IEEE Trans on Components, Packaging, and Manufacturing Technology-Part B, 21(1):105-114, February 1998.

[4] C. Coelho, J. Phillips, and L. Silveira. Robust Rational Function Approximation Algorithm for Model Generation. In $D A C$, pages 207-212, June 1999.

[5] C. Coelho, J. Phillips, and L. Silveira. A Convex Programming Approach to Positive Real Rational Approximation. In ICCAD, pages 4-8, November 2001.

[6] C. Coelho, J. Phillips, and L. Silveira. Optimization Based Passive Constrained Fitting. In the 2002 ICCAD, pages 10-14, San Jose, California, November 2002.

[7] L. Daniel, O. Siong, C. L., K. Lee, and J. White. A multiparameter moment matching model reduction approach for generating geometrically parameterized interconnect performance models. IEEE Trans. on CAD, 23(5):678-693, 2004.

[8] B. Gustavsen and A. Semlyen. Rational Approximation of Frequency Domain Responses by Vector Fitting. IEEE Trans on Power Delivery, 14(3):1052-1061, July 1999.

[9] P. Heydari and M. Pedram. Model reduction of variable-geometry interconnects using variational spectrallyweighted balanced truncation. In ICCAD, San Jose, CA, November 2001.

[10] X. Hu, J. White, and L. Daniel. Analysis of full-wave conductor system impedance over substrate using novel integration techniques. In $D A C$, June 2005.

[11] T. Kailath. Linear Systems. Prentice-Hall, 1980.

[12] M. Kamon, N. Marques, and J. White. FastPep: A Fast Parasitic Extraction Program for Complex ThreeDimension Geometries. In ICCAD, pages 456-460, November 1997.

[13] P. Li, F. Liu, S. Nassif, and L. Pileggi. Modeling interconnect variability using efficient parametric model order reduction. In Design, Automation and Test Conference in Europe, March 2005.

[14] J. Lim and A. Oppenheim. Advanced Topics in Signal Processing. Prentice Hall, 1988.

[15] Y. Liu, L. T. Pileggi, and A. J. Strojwas. Model order-reduction of RCL interconnect including variational analysis. pages 201-206, 1999.

[16] A. Megretski. Positivity of Trigonometric Polynomials. In 42nd IEEE CDC, volume 4, pages 3814-3817, December 2003.

[17] A. Odabasioglu, M. Celik, and L. Pileggi. Prima:Passive reduced-order interconnect macromodeling algorithm. In ICCAD, pages 58-65, November 1997.

[18] J. Peters. Design of High Quality Factor Inductos in RF MCM-D. Master's thesis, Massachusetts Institute of Technology, Cambridge, MA, U.S.A., September 2004. 
[19] C. Prud'homme, D. Rovas, K. Veroy, Y. Maday, A. Patera, and G. Turinici. Reliable real-time solution of parametrized partial differential equations: Reduced-basis output bounds methods. Journal of Fluids Engineering, 2002.

[20] S. Pullela, N. Menezes, and L. Pileggi. Moment-sensitivity-based wire sizing for skew reduction in on-chip clock nets. IEEE Trans. Computer-Aided Design, 16(2):210-215, February 1997.

[21] J. Sturm. Using SeDuMi 1.02, a MATLAB Toolbox for Optimization over Symmetric Cones. Tilburg University, 2001.

[22] D. Vasilyev and J. White. RLE Internal Memorandum, MIT, April 2005.

[23] D. S. Weile, E. Michielssen, E. Grimme, and K. Gallivan. A method for generating rational interpolant reduced order models of two-parameter linear systems. Applied Mathematics Letters, 12:93-102, 1999. 


\title{
Structure Preserving Reduction of Frequency-dependent Interconnect
}

Quming Zhou, Kartik Mohanram, Athanasios C. Antoulas

Department of Electrical and Computer Engineering, Rice University, Houston, TX

\begin{abstract}
A rational Arnoldi method for passivity-preserving model-order reduction (MOR) with implicit multi-point moment matching for systems with frequency-dependent interconnects is described. The structure $\mathbf{H}(s)=s \mathbf{E}-\mathbf{A}-\mathbf{K} \sqrt{ } f$, which arises from frequencydependent effects in high speed interconnects, is preserved by the proposed MOR technique. Moment matching using congruence transforms and based on two types of moments that are derivatives of the transfer function w.r.t $s$ and $\sqrt{ } f$ is described. Simulation results show that the proposed approach can significantly reduce the complexity of systems with frequency-dependent elements, while retaining high accuracy in comparison to the original system in both the time and frequency domains.
\end{abstract}

Keywords: Model-order reduction, skin effect, interconnect

\section{REFERENCES}

[1] L. T. Pillage and R. A. Rohrer, "Asymptotic waveform evaluation for timing analysis," IEEE Trans. CAD, Vol. 9, pp. 352-366, Apr. 1990.

[2] A. Odabasioglu, M. Celik, and L. Pileggi, "PRIMA: Passive reduced-order interconnect macromodeling algorithm," IEEE Trans. CAD, Vol. 17, pp. 645-654, Aug. 1998.

[3] P. Feldmann and R. W. Freund, "Efficient linear circuit analysis by Padé approximation via the Lanczos process," IEEE Trans. CAD, Vol. 14, pp. 639-649, May 1995.

[4] A. C. Antoulas, "A new result on passivity preserving model reduction," Systems and Control Letters, Vol. 54, pp. 361-374, Apr. 2005.

[5] H. Zheng and L. T. Pileggi, "Robust and passive model order reduction for circuits containing susceptance elements," Proc. ICCAD, pp. 761-766, 2002.

[6] R. W. Freund, "SPRIM: Structure-preserving reduced-order interconnect macromodeling," Proc. ICCAD, pp. 80-87, 2004.

[7] Y. Su, et al., "SAPOR: Second-order Arnoldi method for passive order reduction of RCS circuits," Proc. ICCAD, pp. 74-79, 2004.

[8] S. Mei, C. Amin, and Y. Ismail, "Efficient model-order reduction including skin effect," Proc. DAC, pp. 232237, 2003.

[9] R. Achar, M. S. Nakhla, and Q. Zhang, "Full-wave analysis of high-speed interconnects using complex frequency hopping," IEEE Trans. CAD, Vol. 17, pp. 997-1016, Oct. 1998.

[10] J. M. Wang, et al., "On projection-based algorithms for model-order reduction of interconnects," IEEE Trans. Circuits and Systems-I, Vol. 49, pp. 1563-1585, Nov. 2002.

[11] I. M. Elfadel and D. D. Ling, "A block rational Arnoldi algorithm for multi-point passive model-order reduction of multiport RLC networks," Proc. ICCAD, pp. 66-71, 1997.

[12] E. J. Grimme, "Krylov projection methods for model reduction," Ph.D. thesis, University of Illinois at UrbanaChampaign, 1997.

[13] R. W. Freund, "Passive reduced-order models for interconnect simulation and their computation via Krylovsubspace algorithms," Proc. DAC, pp. 195-200, 1999. 


\title{
Segregation by Primary Phase Factors: A Full-wave Algorithm for Model Order Reduction
}

Thomas J. Klemas, Luca Daniel, Jacob K. White

Research Laboratory for Electronics, Massachusetts Institute of Technology, Cambridge MA

\begin{abstract}
Existing Full-wave Model Order Reduction (FMOR) approaches are based on Expanded Taylor Series Approximations (ETAS) of the oscillatory full-wave system matrix. The accuracy of such approaches hinges on the worst case interaction distances, producing accurate models over a very narrow band of frequencies. In this paper we present Segregation by Primary Phase Factors (SPPF), a novel algorithm for FMOR enabling wideband interconnect impedance analysis. SPPF extracts exponential terms (primary phase factors) and then approximates the smoother remainder with an ETAS, thus resulting in good accuracies over a very wide band of frequencies. We also present a technique to improve conditioning for the required computation. Example results are given for simple interconnect structures modeled using a discretized mixed potential integral equation formulation.
\end{abstract}

Keywords: Full-wave Impedance Extraction, Model Order Reduction

\section{REFERENCES}

[1] C. P. Coelho, J. Phillips, and L. M. Silveira. A convex programming approach for generating guaranteed passive approximations to tabulated frequency-data. IEEE Transactions CAD on Integrated Circuits and Systems, 23(2):293-301, Feb 2004.

[2] P. Feldmann and R. W. Freund. Efficient linear circuit analysis by Padé approximations via the Lanczos process. In EURO-DAC'94 with EURO-VHDL'94, September 1994.

[3] E. Grimme. Krylov Projection Methods for Model Reduction. PhD thesis, Coordinated-Science Laboratory, University of Illinois at Urbana-Champaign, Urbana-Champaign, IL, 1997.

[4] R. F. Harrington. Field Computation by Moment Methods. MacMillan, New York, 1968.

[5] H. Heeb and A. E. Ruehli. Three-dimensional interconnect analysis using partial element equivalent circuits. IEEE Trans. On Circuits and Systems I: Fundamental Theory and Applications, 39(11):974-982, November 1992.

[6] M. Kamon, N. Marques, and J. K. White. FastPep: a fast parasitic extraction program for complex threedimensional geometries. In Proc. of the IEEE/ACM International Conference on Computer-Aided Design, pages 456-460, San Jose, CA, Nov. 1997.

[7] S. Kapur and D. E. Long. Ies3: efficient electrostatic and electromagnetic simulation. Computational Science and Engineering, IEEE, 5(4):60-67, Oct-Dec 1998.

[8] T. J. Klemas, L. Daniel, and J. White. A fast full-wave algorithm to generate low order electromagnetic scattering models. In Proceedings IEEE APS-URSI Symposium. IEEE Antennas and Propagation Society/URSI, July 2005.

[9] K.Willcox and J.Peraire. Balanced model reduction via the proper orthogonal decomposition. AIAA Journal, 40(11):2323-2330, November 2002.

[10] K. Nabors and J. K. White. FastCap: a multipole accelerated 3-d capacitance extraction program. IEEE Trans. on Computer-Aided Design of Integrated Circuits and Systems, 10(11):1447-59, Nov. 1991.

[11] J. R. Phillips, E. Chiprout, and D. D. Ling. Efficient full-wave electromagnetic analysis via model-order reduction of fast integral transforms. In Proceedings 33rd Design Automation Conference, Las Vegas, Nevada, June 1996.

[12] J. R. Phillips and J. K. White. A Precorrected-FFT method for electrostatic analysis of complicated 3-D structures. IEEE Trans. on Computer-Aided Design of Integrated Circuits and Systems, 16(10):1059-1072, Oct. 1997.

[13] J. C. Rautio and R. F. Harrington. An electromagnetic time-harmonic analysis of shielded microstrip circuit. IEEE Trans. Microwave Theory Tech., MTT-35:726-730, 1987.

[14] A. E. Ruehli. Equivalent Circuit Models for Three-Dimensional Multiconductor Systems. IEEE Transactions on Microwave Theory and Techniques, MTT-22(3):216-221, March 1974.

[15] L. M. Silveira, M. Kamon, and J. K. White. Direct computation of reduced-order models for circuit simulation of 3-d interconnect structures. In Proceedings of the 3rd Topical Meeting on Electrical Performance of Electronic Packaging, pages 254-248, Monterey, California, November 1994. 
[16] Z. Zhu, J. Huang, B. Song, and J. K. White. Algorithms in fastimp: a fast and wideband impedance extraction program for complicated 3-d geometries. In Proc. of the IEEE/ACM Design Automation Conference, Los Angeles, CA, June 2003. 